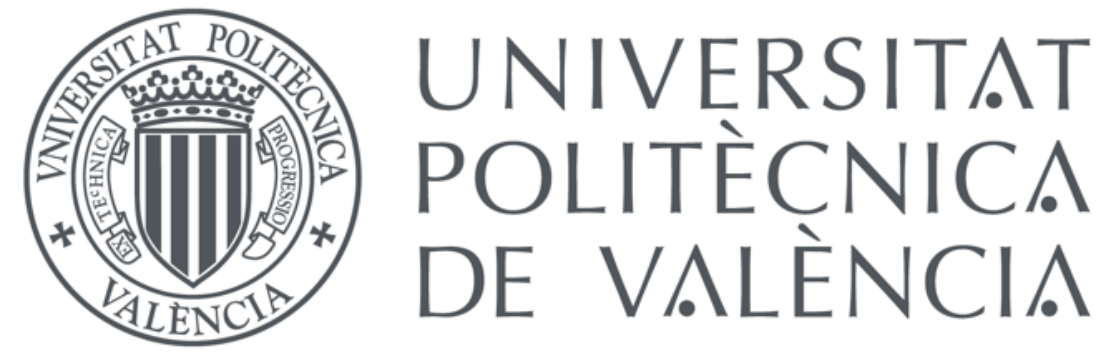

Doctoral Thesis

\title{
Development and Performance Evaluation of High Resolution TOF- PET Detectors Suitable for Novel PET Scanners
}

\section{Efthymios Lamprou}

Under the supervision of

Antonio J. González Martínez

Valencia, January 2021 


\section{Doctoral Thesis}

Development and Performance Evaluation of High Resolution TOF-PET Detectors Suitable for Novel PET Scanners

Author: Efthymios Lamprou

Director: Antonio J. González Martinez

Tribunal:

President: Geoge Loudos

Vocal: Paul Lecoq

Secretary: David Gascon Fora

External evaluators:

Javier Vijande Asenjo

David Gascon Fora

Gerard Ariño Estrada

Grade: Excellent (10/10), Cum Laude

Defense date: Friday, 29 of January, 2021

Valencia, Spain 


\section{Summary}

Positron Emission Tomography (PET) is one of the greatest tools of modern diagnostic medicine and the most representative in the field of molecular imaging. This imaging modality, is capable of providing a unique type of functional information which permits a deep visualization, quantification and understanding of a variety of diseases and pathologies. Areas like oncology, neurology, or cardiology, among others, have been well benefited by this technique. Although numerous important advances have already been achieved in PET, some other individual aspects still seem to have a great potential for further investigation. One of the main trends in modern PET research and development, is based in the extrapolation of the TimeOf-Flight (TOF) information from the gamma-ray detectors. In such case, an increase in the effective sensitivity of PET is accomplished, resulting in an improved image signal-to-noise ratio. However, the direction towards a precise decoding of the photons time arrival is a challenging task that requires, besides specific approaches and techniques, tradeoffs between cost and performance. A performance characteristic very habitually compromised in TOF-PET detector configurations is the spatial resolution. As it will be discussed, this feature is directly related to the scintillation materials and types, and consequently, with system cost and complexity.

In this thesis, motivated by the well-known benefits in clinical imaging of a precise time and spatial resolution, we propose novel TOF-PET detector configurations capable of inferring both characteristics. Our suggestions are based in light sharing approaches, either using monolithic detectors or crystal arrays with different pixel-to-photosensor sizes. These approaches, make it possible to reach a precise impact position determination. However, their TOF capabilities have not yet been explored in depth. In the present thesis, through a series of peer-reviewed publications we attempt to demonstrate the challenges encountered in these kinds of configurations, propose specific approaches improving their performance and eventually reveal their limits in terms of timing.

High emphasis is given in analyzing and studying the scintillation light distributions and their impact to the timing determination. To the best of our knowledge, this is one of the first works in which such detailed study of the relation between light distribution and timing capabilities is carried out, especially when using analog SiPMs and ASICs. Hopefully, this thesis will motivate and enable many other novel design concepts, useful in PET instrumentation as well as it will serve as a helpful reference for similar attempts.

The present $\mathrm{PhD}$ thesis is organized as follows. There is an introduction part composed by three detailed sections. We attempt to summarize here some of the knowledge related to PET imaging and especially with the technique of TOF-PET. Some very recent but still unpublished results are also presented and included in this part, aiming to support statements and theories. The second part of this thesis lists the four peer-reviewed papers that the candidate is including. 
La Tomografía por Emisión de Positrones (PET) es una de las técnicas más importantes en la medicina de diagnóstico actual y la más representativa en el campo de la Imagen Molecular. Esta modalidad de imagen es capaz de producir información funcional única, que permite la visualización en detalle, cuantificación y conocimiento de una variedad de enfermedades y patologías. Áreas como la oncología, neurología o la cardiología, entre otras, se han beneficiado en gran medida de esta técnica. A pesar de que un elevado número de avances han ocurrido durante el desarrollo del PET, existen otros que son de gran interés para futuras investigaciones. Uno de los principales pilares actualmente en PET, tanto en investigación como en desarrollo, es la obtención de la información del tiempo de vuelo (TOF) de los rayos gamma detectados. Cuando esto ocurre, aumenta la sensibilidad efectiva del PET, mejorando la calidad señal-ruido de las imágenes. Sin embargo, la obtención precisa de la marca temporal de los rayos gamma es un reto que requiere, además de técnicas y métodos específicos, compromisos entre coste y rendimiento. Una de las características que siempre se ve afectada es la resolución espacial. Como discutiremos, la resolución espacial está directamente relacionada con el tipo de centellador y, por lo tanto, con el coste del sistema y su complejidad.

En esta tesis, motivada por los conocidos beneficios en imagen clínica de una medida precisa del tiempo y de la posición de los rayos gamma, proponemos configuraciones de detectores TOFPET novedosos capaces de proveer de ambas características. Sugerimos el uso de lo que se conoce como métodos de "light-sharing", tanto basado en cristales monolíticos como pixelados de tamaño diferente al del fotosensor. Estas propuestas hacen que la resolución espacial sea muy alta. Sin embargo, sus capacidades temporales han sido muy poco abordadas hasta ahora. En esta tesis, a través de varios artículos revisados, pretendemos mostrar los retos encontrados en esta dirección, proponer determinadas configuraciones y, además, indagar en los límites temporales de éstas.

Hemos puesto un gran énfasis en estudiar y analizar las distribuciones de la luz centellante, así como su impacto en la determinación temporal. Hasta nuestro conocimiento, este es el primer trabajo en el que se estudia la relación de la determinación temporal y la distribución de luz de centelleo, en particular usando SiPM analógicos y ASICs. Esperamos que esta tesis motive y permita otros muchos trabajos orientados en nuevos diseños, útiles para instrumentación PET, así como referencia para otros trabajos.

Esta tesis esta organizada como se describe a continuación. Hay una introducción compuesta por tres capítulos donde se resumen los conocimientos sobre imagen PET, y especialmente aquellos relacionados con la técnica TOF-PET. Algunos trabajos recientes, pero aún no publicados se muestran también, con el objetivo de corroborar ciertas ideas. En la segunda parte se incluyen las cuatro contribuciones que el candidato sugiere para el compendio de artículos. 
La Tomografia per Emissió de Positrons (PET) és una de les tècniques més importants en la medicina de diagnòstic actual i la més representativa en el camp de la Imatge Molecular. Esta modalitat d'imatge és capaç de produir informació funcional única, que permet la visualització en detall, quantificació i coneixement d'una varietat de malalties i patologies. Àrees com l'oncologia, neurologia o la cardiologia, entre altres, s'han beneficiat en gran manera d'aquesta tècnica. Tot $\mathrm{i}$ que un elevat nombre d'avanços han ocorregut durant el desenvolupament del PET, hi ha altres que són de gran interés per a futures investigacions. Un dels principals pilars actuals en PET, tant en investigació com en desenvolupament, és l'obtenció de la informació del temps de vol (TOF en anglès) dels raigs gamma detectats. Quan açò ocorre, augmenta la sensibilitat efectiva del PET, millorant la qualitat senyal-soroll de les imatges. No obstant això, l'obtenció precisa de la marca temporal dels raigs gamma és un repte que requerix, a més de tècniques i mètodes específics, compromisos entre cost i rendiment. Una de les característiques que sempre es veu afectada és la resolució espacial. Com discutirem, la resolució espacial està directament relacionada amb el tipus de centellador, i per tant, amb el cost del sistema i la seua complexitat.

En aquesta tesi, motivada pels coneguts beneficis en imatge clínica d'una mesura precisa del temps i de la posició dels raigs gamma, proposem nouves configuracions de detectors TOF-PET capaços de proveir d'ambduess característiques. Suggerim l'ús del que es coneix com a mètodes de "light-sharing", tant basat en cristalls monolítics com pixelats de diferent tamany del fotosensor. Aquestes propostes fan que la resolució espacial siga molt alta. No obstant això, les seues capacitats temporals han sigut molt poc abordades fins ara. En aquesta tesi, a través de diversos articles revisats, pretenem mostrar els reptes trobats en aquesta direcció, proposar determinades configuracions i, a més, indagar en els límits temporals d'aquestes.

Hem posat un gran èmfasi a estudiar i analitzar les distribucions de la llum centellejant, així com el seu impacte en la determinació temporal. Fins al nostre coneixement, aquest és el primer treball en què s'estudia la relació de la determinació temporal i la distribució de llum de centelleig, en particular utilitzant SiPM analògics i ASICs. Esperem que aquesta tesi motive i permeta molts altres treballs orientats en nous dissenys, útils per a instrumentació PET, així com referència per a altres treballs.

Aquesta tesi esta organitzada com es descriu a continuació. Hi ha una introducció composta per tres capítols on es resumeixen els coneixements sobre imatge PET i, especialmente, aquells relacionats amb la tècnica TOF-PET. Alguns treballs recents, però encara no publicats es mostren també, amb l'objectiu de corroborar certes idees. La segona part de la tesi conté els quatre articles revisats que el candidat suggereix. 


\section{Acknowledgements}

The present $\mathrm{PhD}$ thesis, is the outcome of many efforts spent towards fulfilling personal ambitious and goals. However, it would not have been realized if it was not for some specific people who kindly provided me with continuous support at all levels, making this journey unique and quite smooth. At this very moment, and before closing this fascinating chapter of my career, I would like to explicitly express my gratitude to all of them.

First and foremost, I would like to thank my supervisor Antonio Javier Gonzalez for offering me the opportunity back in 2016 to move to Spain and join his team. I am highly grateful to him for his willingness to provide me all insights of PET and for making me a better scientist and human. Constantly motivated, he led the whole team, including me, by example and inspired our research efforts. Honestly, it was a privilege to work with him and learn by his side. I hope we may continue to strengthen our collaboration, while we keep on spending quality time inside and outside of the lab, including the tennis courts.

In the same direction, I would like to express my gratitude towards the I3M director José María Benlloch, who truly believed in me and continually supporting my efforts. Additionally, I am very thankful to him for ensuring me a financial aid through a local grant, which made this $\mathrm{PhD}$ possible. I would also like to thank Filomeno Sanchez, the senior scientist who was always available for long and fruitful discussions. His willingness and kindness to share his valuable experience and knowledge were proven to be extremely helpful for my research career.

Along with them, I would like to thank all current and former members of PET team. Apart from their contributions in my research lines, from the very first day they facilitated my integration to the laboratory and all together we created a highly efficient team environment which was soon translated to successes and important accomplishments. Koldo, Liczandro, Gabriel, Andrea, Marta, Celia, Sergio, John, Neus, Adrian, Sara, Georgios, Jose Maria Monzo, thank you all guys for your inputs and help; it was nice to work with you. I feel I own a special thanks to Albert, a former i3M member who introduced me to the fascinating world of TOF-PET. Although our collaboration was short, it was fun spending time struggling together. I would also like to thank former colleagues from Oncovision and Bruker, especially Julio, who is always willing to patiently share his knowledge with me.

On personal level, I am profoundly grateful to all my family members for being deeply supportive and always by my side. First of all, to my parents, Nikos and Alexandra and to my sister Vasia for their timely encouragement and unconditional trust. Their love and support are priceless to me and I know that I wouldn't have made it without them. To my uncles Spiros and Giannis, for their continuous guidance and helpful advices. To all my cousins, who have been 
always there for me. Last but not least, to my beloved grandmothers Evangelia and Niki, for their unconditional love. Thank you all for supporting my dreams!

Moreover, I would like to thank my companion in life, Isabel, for being highly supportive and caring. Her constantly positive attitude and encouragement made the writing of this thesis much smoother than it meant to be. Thank you for your love and for making each day unique. Same for her amazing family that always takes care of making each weekend special.

Thinking about friends, I realize how lucky I am to be surround from great people who always are there for me when I need them. Some of them pretty close, others in countless kilometers away, all have in common their willing and gift to provide me with an excellent escape from the everyday routine. Thank you all, for your constant motivation and support and please, forgive me for not naming you individually. I will keep counting on you guys.

Before concluding, I would like to acknowledge all people I had the chance to meet through collaborations, placements, conferences and travels. Thank you all, for the knowledge sharing, for the time spent in laboratories and of course for all unique fun moments we had together. 


\section{Table of contents}

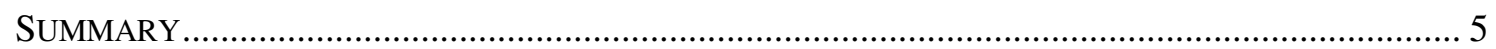

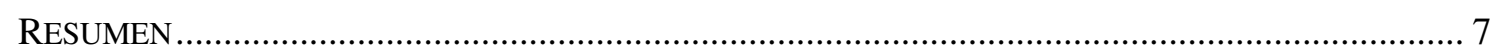

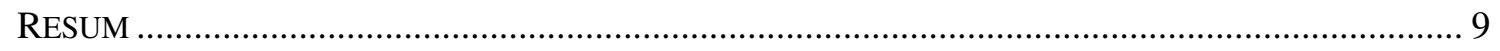

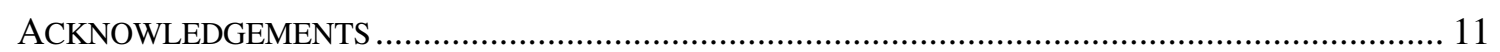

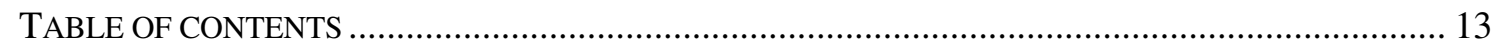

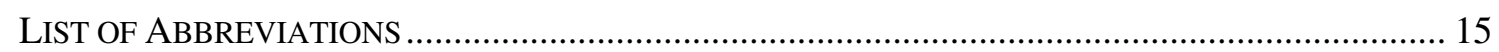

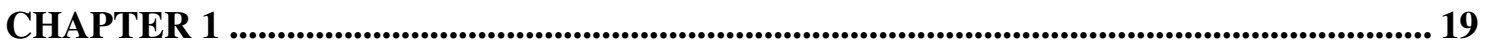

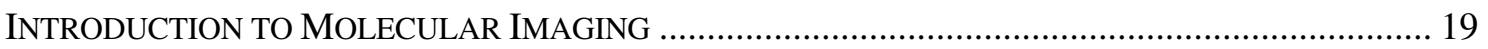

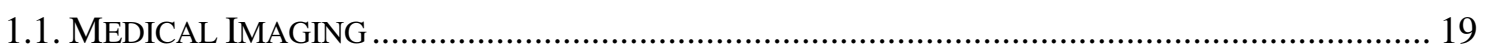

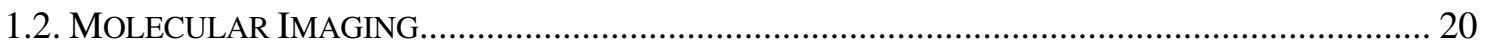

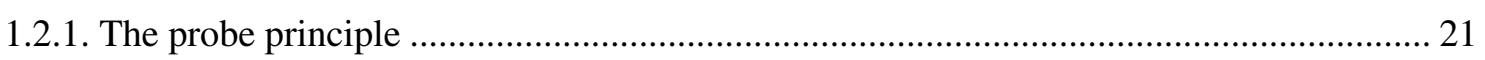

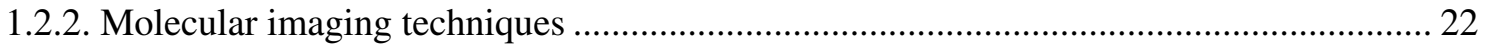

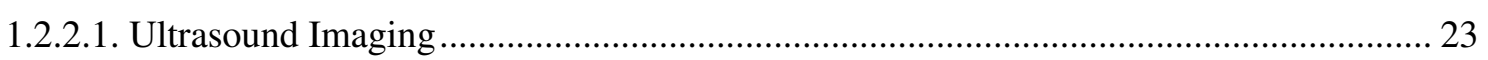

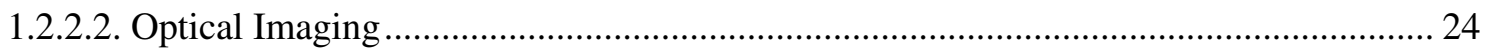

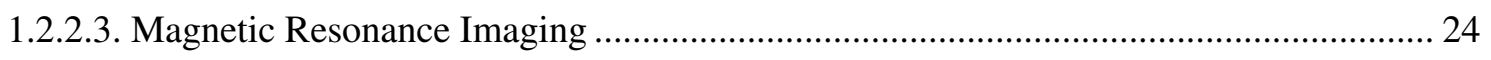

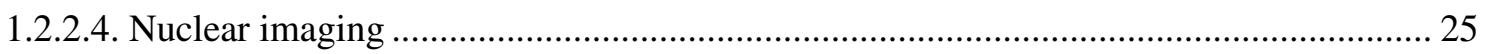

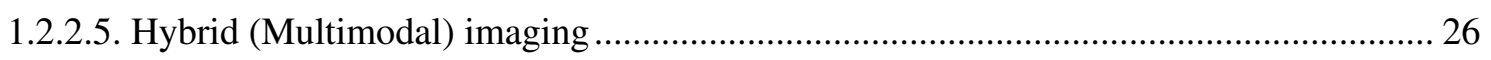

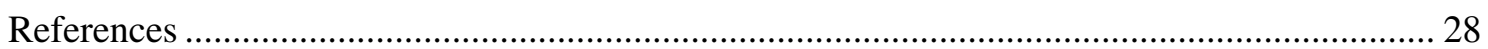

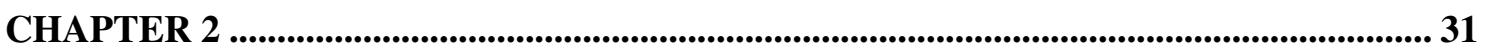

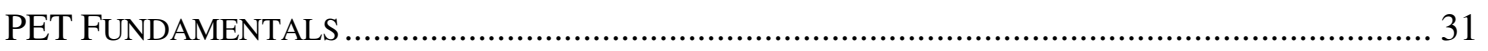

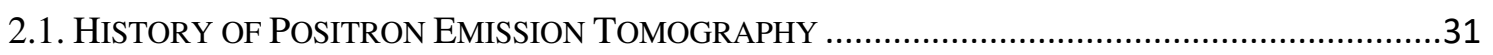

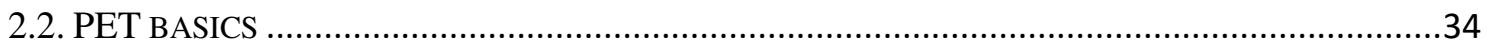

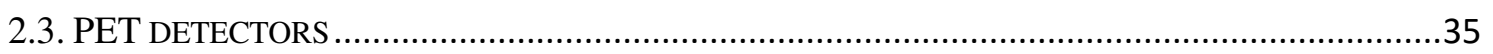

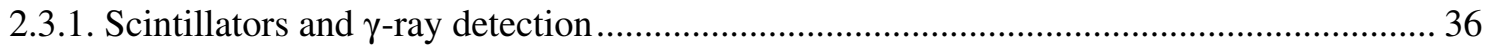

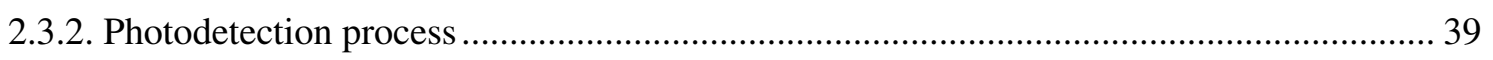

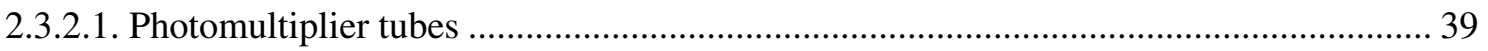

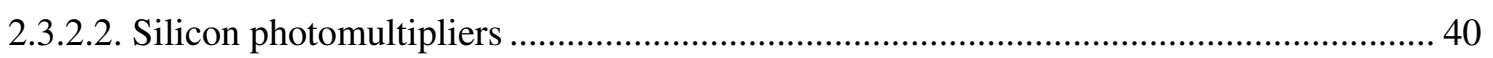

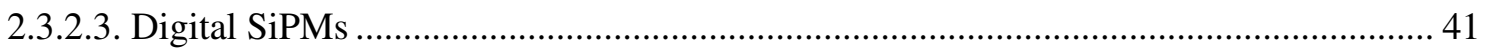

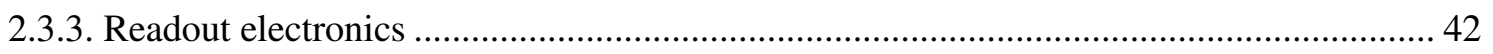

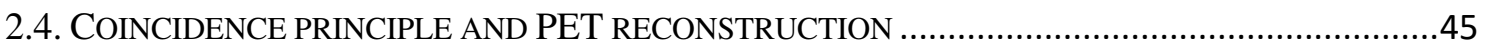

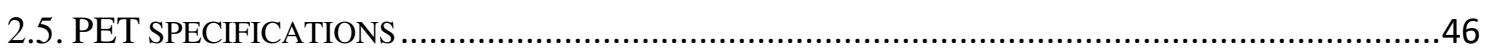

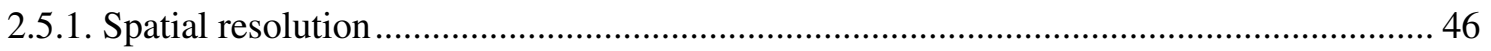

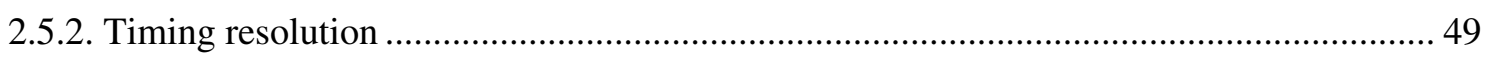




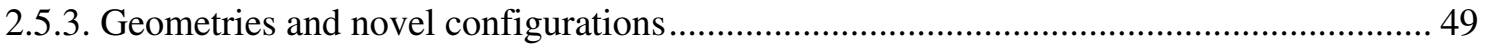

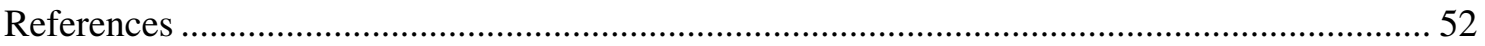

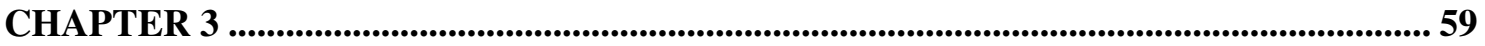

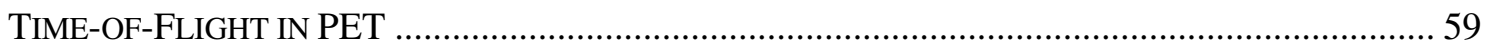

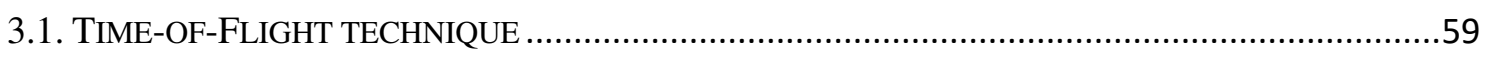

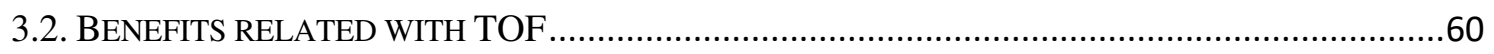

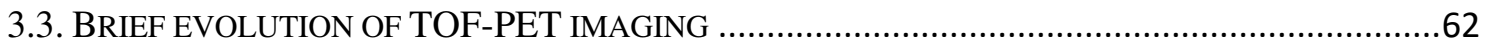

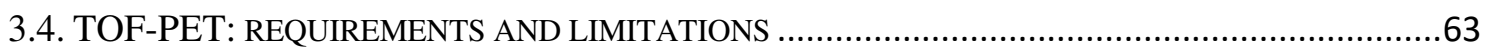

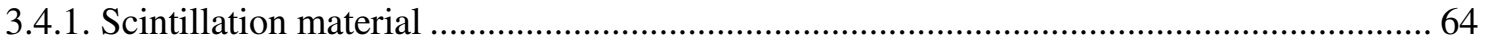

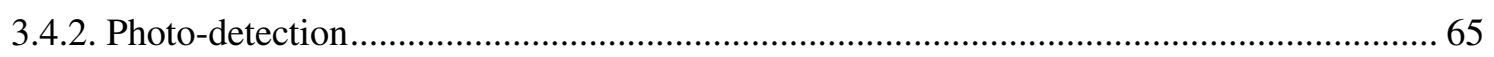

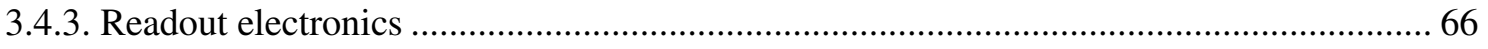

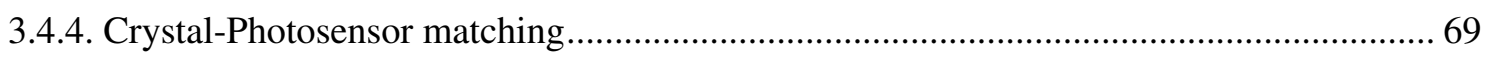

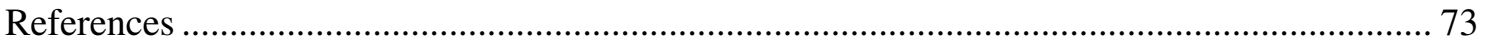

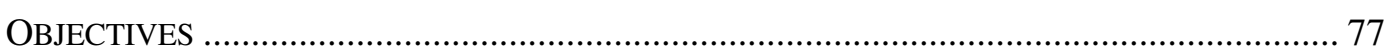

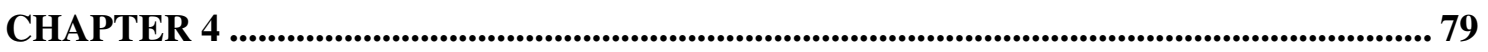

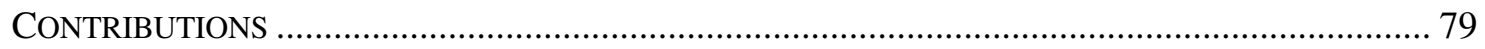

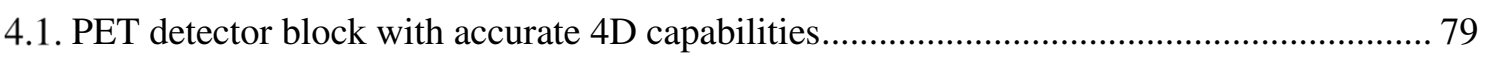

4.2. TOF-PET Detectors Based on ASIC Technology and Analog SiPMs ................................ 89

4.3. Exploring TOF capabilities of PET detector blocks based on large monolithic crystals and

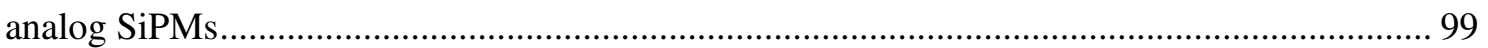

4.4. In-depth evaluation of TOF-PET detectors based on crystal arrays and the TOFPET2 ASIC

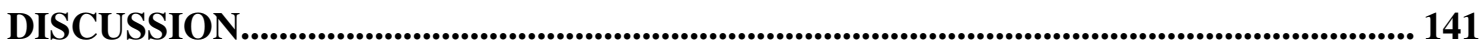

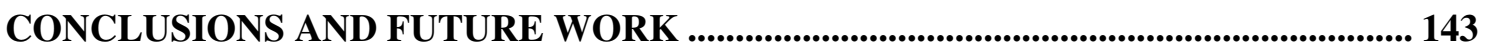

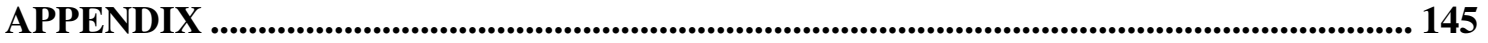


ADC

ASIC

BPF

CFD

CMOS

CPU

CT

CTR

DCR

DOI

DTR

FBP

FDG

FLT

FOV

GPU

LAT

LED

LOR

LTE

LY

MI

MLEM

MRI

OSEM

OTTS

PDE

PET

PMT

ROI

SiPM

SNR
Analog-to-Digital-Converter

Application-Specific-Integrated-Circuit

Back Projection-Filtering

Constant Fraction Discriminator

Complementary Metal-Oxide-Semiconductor

Central Processor Unit

Computed Tomography

Coincidence Time Resolution

Dark Count Rate

Depth-Of-Interaction

Detector Time Resolution

Filtered-Back Projection

Fluorodeoxyglucose

Fluorothymidine F-18

Field-Of-View

Graphical Processor Unit

Limited Angle Tomography

Leading-Edge Discriminator

Line-Of-Response

Light Transfer Efficiency

Light Yield

Molecular Imaging

Maximum Likelihood-Expectation Maximization

Magnetic Resonance Imaging

Ordered subsets Expectation Maximization

Optical-Transit-Time-Spread

Photodetection Efficiency

Positron Emission Tomography

Photomultiplier Tube

Region of Interest

Silicon Photomultiplier

Signal-To-Noise Ratio 
SPAD

SPECT

SPTR

TDC

TOF

TOT

VOI
Single Photon Avalanche Diode

Single Photon Emission Computed Tomography

Single Photon Time Resolution

Time-To-Digital Converter

Time-Of-Flight

Time Over Threshold

Volume of Interest 
$\Sigma \tau \eta v \alpha v i \psi(\alpha \dot{\alpha} \mu o v A \lambda \varepsilon \xi \alpha \dot{v} v \delta \rho \alpha .$. 


\section{Chapter 1}

\section{| \\ Introduction to Molecular Imaging}

The discovery of x-rays by Wilhelm Rontgen back in 1895, highly impressed the world and essentially laid the foundation of diagnostic radiology. Since then, the whole concept of medical imaging has expanded and improved immensely. Today we barely get fascinated by the way images are generated as it is so ubiquitous. However, behind each medical equipment, a great number of discoveries and developments has been involved, and many engineering innovations and solutions were required. In the present chapter we will briefly introduce molecular imaging; a fascinating aspect of the modern diagnostic practice.

\subsection{Medical Imaging}

Medical Imaging consists an essential component of the care pathway of the modern society with more than 5 billion imaging studies conducted already worldwide [1]. Since the appearance of the first scanner and up to the modern era, the goal of Medical Imaging has always been the enhancement of the non-invasive diagnostic processes, and the establishment of an accurate and personalized treatment, accessible to everyone [2].

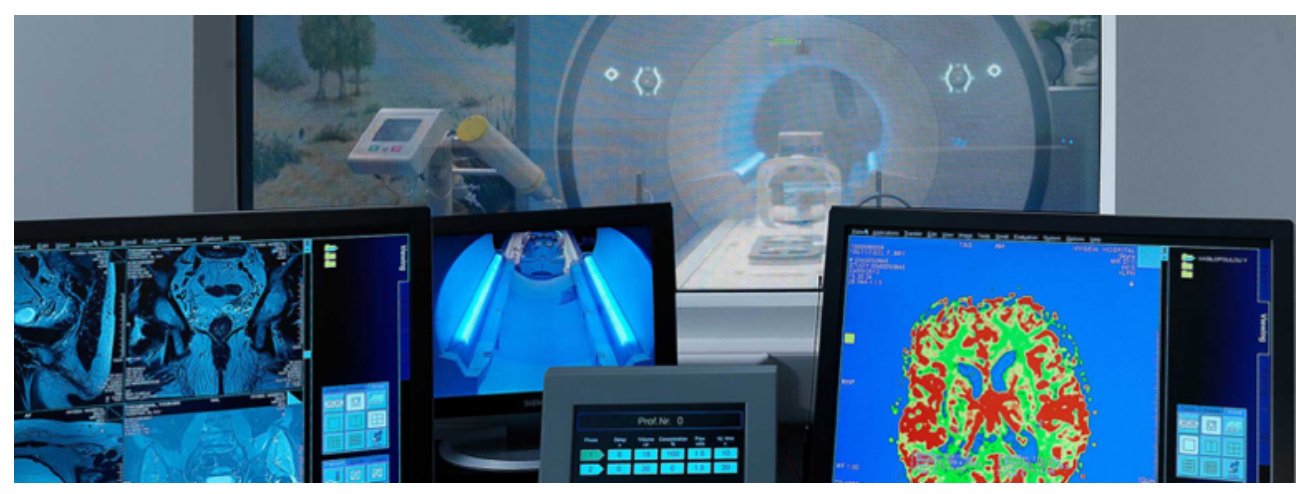

Figure 1.1. View from a medical imaging control room (extracted from www.hygeia.gr).

In the clinical context, the term Medical Imaging refers to a plethora of imaging techniques [3]. Nowadays, numerous and diverse techniques exist and serve the society. An example of a modern control imaging room is shown in Figure 1.1. Although an accurate categorization of all 
imaging modalities could result laborious, an effective classification relies on the type of information they are capable of providing [4]. The in-vivo information currently exploited in the modern clinical diagnostic practice can be divided into anatomic and functional.

The anatomic information, also referred as structural, is essentially the detailing of internal structures and tissues and the detection of phenotypic alterations inside the object of study. This is achieved by determining inherent differences in tissue contrast to locate and measure the magnitude of the pathology. Techniques such as X-Rays, Computed Tomography (CT) or Magnetic Resonance Imaging (MRI), are excellent in revealing gross anatomic manifestations with extremely high resolution (see Figure 1.2). However, when it comes to provide insights into specific diseases, they typically lack of efficiency.

Functional information reveals physiological and biochemical processes that occur both at molecular and cellular levels [4]. This information, originated from the field of Molecular Imaging (MI), is embracing unique advances related to early diagnosis, treatment follow up, and pharmacological discoveries. Currently, MI lavishly provides this type of information through its various techniques, forming a field with great potential and significance [5].

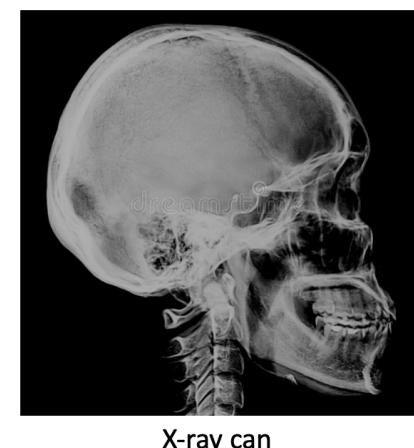

(a)

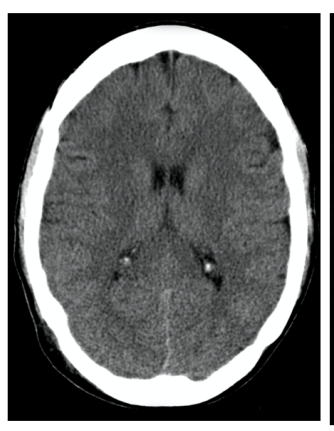

CT Scan

(b)

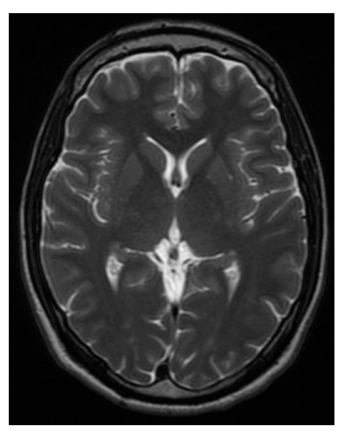

(c)

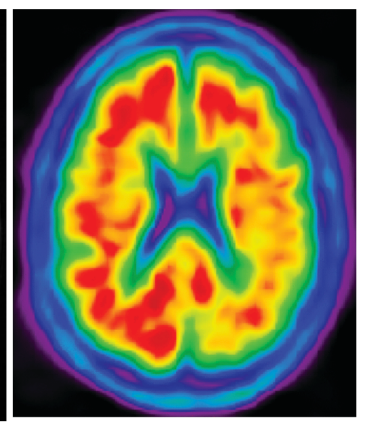

PET Scan

(d)

Figure 1.2. Examples of diagnostic imaging information: (a) head x-ray, (b) brain Computed Tomography scan, (c) magnetic resonance (T2 weighted) brain imaging and last (d) brain PET scan. (a), (b) and (c) were extracted from Wikipedia while (d) was extracted from [6].

\subsection{Molecular Imaging}

Molecular Imaging arose in the mid twentieth century as a discipline at the intersection of molecular biology and in-vivo imaging, transforming the clinical and preclinical diagnostic processes. It has been defined as the non-invasive visualization and quantification of the biological processes taking place at cellular and subcellular levels in living subjects [7]. In other words, MI provides access to a unique information regarding the biochemical activities of an underlying disease. Through this information, physicians and researchers not only can diagnose with more accuracy molecular abnormalities, but they can also study and better understand a 
disease from its basis. In contrast to this, classical anatomic imaging modalities are mainly limited to just reveal the end-effects of these molecular alterations (Figure 1.2).

Apart from the great benefit of the early diagnosis, MI procedures can be exploited in several more ways. For instance, they are good alternatives to more invasive diagnostic methods such as biopsies or surgical processes. Additionally, MI techniques serve as excellent tools in treatment and therapy assessment tasks. Herein, by having access to the aforementioned functional information, physicians can more efficiently schedule and adapt each treatment plan based on the molecular abnormalities of each individual case while at the same time they can better monitor and follow-up each strategy, evaluating its effectiveness even shortly after its application (Figure 1.3). Finally, MI is suggested as an unprecedented tool for all studies related with drug discovery and evaluation, due to the fact that a better understanding of diseases and drug distribution inside a living organism can be obtained.
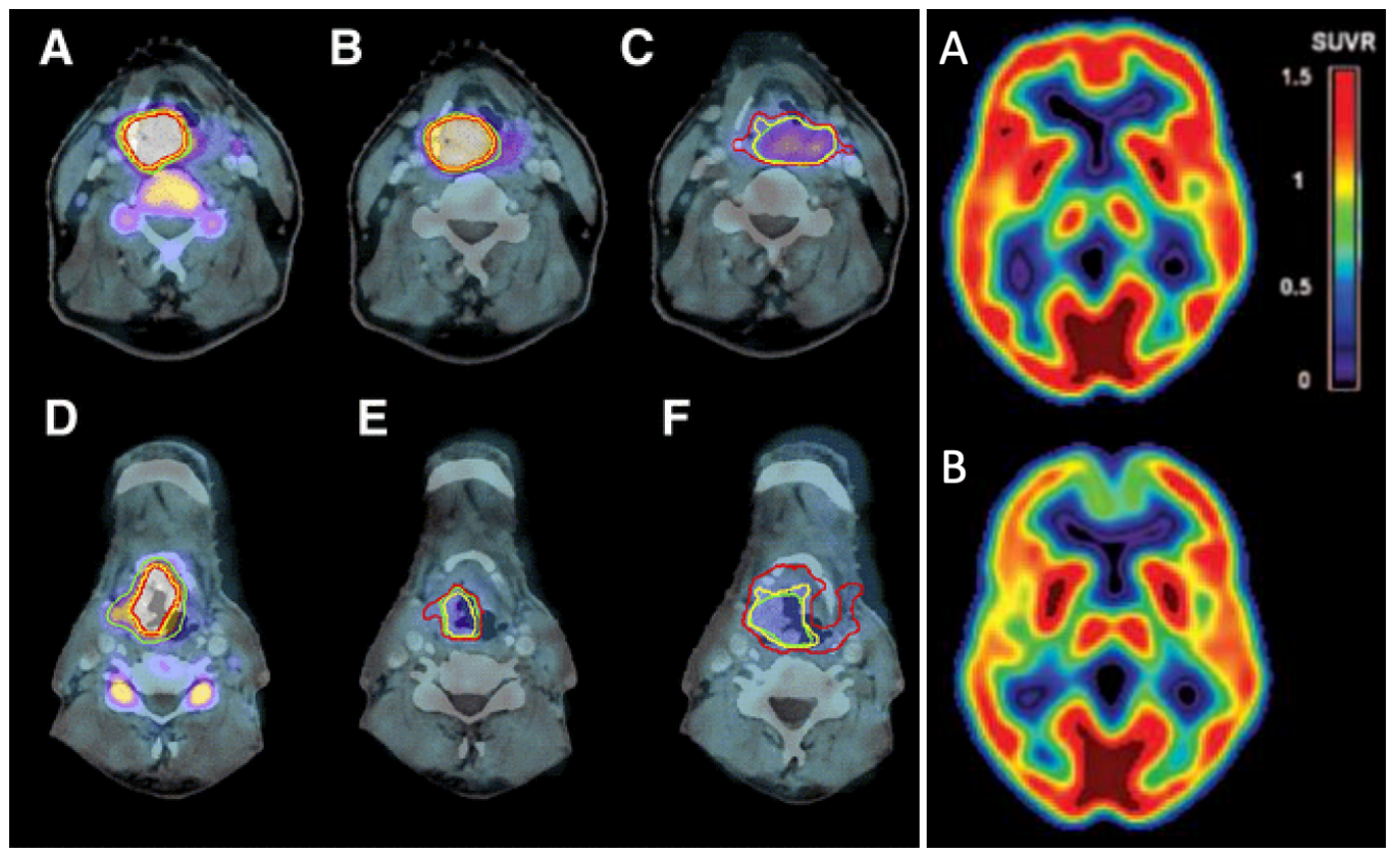

Figure 1.3. Left: PET/CT before therapy (A,D), in 2nd week of therapy (B, E) and in 4th week (C,F). Top row shows slow decrease in probe uptake in patient treated for supraglottic laryngeal carcinoma. Bottom row shows a patient with fast decrease of FLT uptake, associated with better survival probability. Extracted from [8]. Right: Top, Normal FDG uptake during a PET brain scan, showing low probability for possible development of Alzheimer disease. Bottom, Abnormal FDG uptake during a PET brain scan, revealing a high-risk case for Alzheimer disease. Extracted and modified from [9].

\subsubsection{The probe principle}

The great potential of MI is derived from the use of special class of pharmaceuticals known as imaging probes. These probes following their administration to the object of study, are directed to specific biological targets or pathways and serve for interacting with the target of interest and visualize the cellular activity [10][11]. These probes essentially are active compounds carrying a 
targeting moiety (a factor that is included in the biological process), a signal agent and lastly a linker that connects the targeting moiety and the agent, see sketch explanation in Figure 1.4.

The signal agent, essentially is a detectable tracer that emits a type of signal for imaging purposes. Depending on the imaging modality, this tracer can be a radionuclide (nuclear imaging), bioluminescence or fluorescent molecules (optical imaging), magnetic molecules (magnetic resonance), or microbubbles (ultrasound). Regarding the moiety, this can be any ligand like small molecules, peptides, protein, antibodies and nanoparticles among others. Moreover, the linker is used in order to couple the agent with the moiety, while typically has a considerable effect on the biodistribution of the imaging probe [12].

Undeniably, molecular probes play a key role in the molecular imaging field. Several scientific disciplines are joint to design, develop and evaluate new probe concepts. Regarding the goals of new probe discoveries, these are well defined and among others include: high binding affinity, specificity to the target, detection sensitivity and of course accessibility. Each new development, broadens molecular imaging applicability, providing an additional tool in the diagnostic practice.

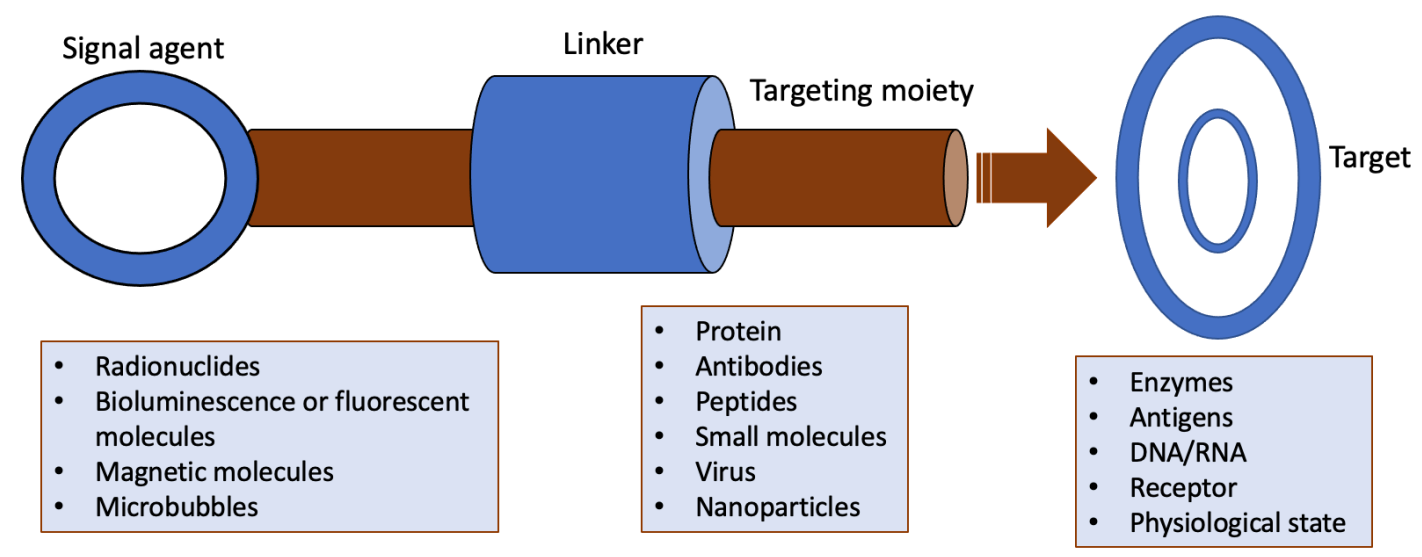

Figure 1.4. Schematic representation of molecular imaging probe along with typically used substances.

\subsubsection{Molecular imaging techniques}

As described above, MI has transformed the way physicians are able to diagnose a wide range of diseases. The suitability of each one of these imaging techniques for each clinical case, strongly depends on factors related with the characteristics of the imaging modality itself. The main factors are the spatial resolution, specific sensitivity and penetration of the biological process, Field-ofView (FOV), and the availability of suitable probes and labels than can be delivered to the imaging target [11], to name but a few. Based on these factors, some specific modalities are preferred over others, while in several cases, one technique may compliment another one. Aiming to complete the background knowledge related with molecular imaging, a brief description of the 
main modalities is presented in the following subsections, while some examples of commercially available MI systems are shown in Figure 1.5.

\subsubsection{Ultrasound Imaging}

Ultrasound imaging has been exploited in the clinical practice for more than 20 years, both as a diagnostic but also as a therapeutic tool. Its principle is based on high-frequency sound waves which interact with soft tissues and organs [13]. These waves, are emitted by probes named transducers typically made of special ceramic crystal materials called piezoelectrics. After several interactions inside the object of study, ultrasound echoes are reflected back and detected again by the transducers. The detection of echoes results in the generation of electrical pulses which after the proper processing, leads to the generation of ultrasound images.

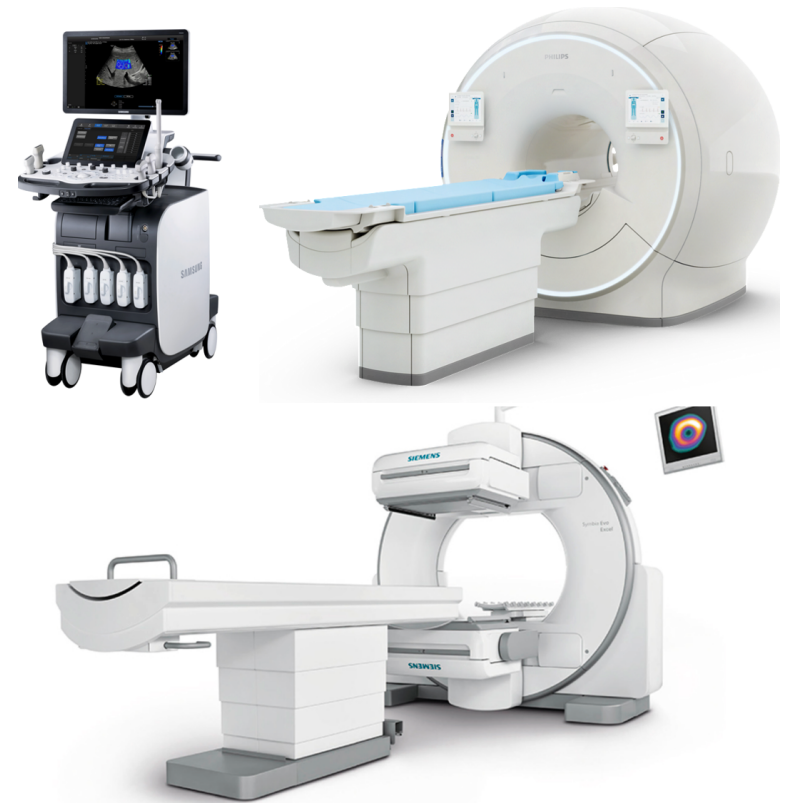

Figure 1.5. Examples of Molecular Imaging systems. Top-left: RS85 ultrasound equipment, commercialized by Samsung (samsunghealthcare.com). Top-right: MRI scanner, distributed by Phillips as Ingenia Elition 3.0T (philips.com). Bottom: A hybrid SPECT-CT imaging scanner named Symbia Intevo Bold by Siemens (siemenshealthineers.com).

Ultrasound-based imaging has been established as a basic diagnostic tool for several types of pathologies, as it shows several advantages over other imaging modalities. Firstly, the continuous improvement in the field of contrast agents and the introduction of new types (e.g liposomes, nanoparticles), has broaden the spectrum of applications in which ultrasounds can be utilized. Additionally, in terms of performance, ultrasounds permit the generation of real time images, with resolutions that range from 50 to 500 micrometers (depending on the operating frequency). Lastly, the low cost and high portability of this imager, make it widely accessible to physicians and thus, to patients. The main limitation of this modality, seems to be the strong and direct dependency of the penetration and spatial resolution with the operating frequency. 
Up to recent years, ultrasounds use was limited in revealing anatomic information from living objects. However, more recently, the introduction of novel specific imaging probes, revealed the molecular dimension of this modality. Hence, this technique permits now the monitoring and visualization of pathological structures and processes. This is possible since these contrast agents are designed to be accumulated at tissue sites overexpressing certain molecular markers.

\subsubsection{Optical Imaging}

Optical imaging is based on the detection of visible and infrared light produced by optical tracers, aiming to visualize and quantify in-vivo biological processes or changes [14]. When photons are emitted, they are transmitted through biological tissues and after a series of possible interactions like photon emission, reflectance, absorption, scattering, or phase-shift, are detected by photodetectors. Following the photon detection, data are processed and the medical images generated.

Optical molecular imaging is described by two techniques namely bioluminescence and fluorescence. Bioluminescent imaging makes use of natural reporters (a typical one is luciferase) which emits light to trace the movement of certain cells or to identify the location of specific chemical reactions within the body. In contrast to this, fluorescence imaging employs proteins that emit light when absorbing an appropriate wavelength of electromagnetic radiation, generated by an external light source such as laser.

Although optical imaging tools have been primarily used in the research field, their clinical use is expected to be significantly expanded over the following years [14]. The main factors that have limited so far this clinical translation, are the low penetration and the depth-dependent attenuation of the emitted light.

\subsubsection{Magnetic Resonance Imaging}

Magnetic Resonance Imaging (MRI) is a powerful tool widely use in modern medicine, as it is capable of delivering high quality structural and functional diagnostic information [15]. The concept of MRI includes a broad variety of techniques, which have led to ever-increasing applications with great benefit for the diagnostic practice. Essentially, MRI systems make use of super conducting magnets which impose strong magnetic fields. These fields result in the alignment of all hydrogen nuclei or protons in the body due to their magnetic moment. Then, through temporary emitted radiofrequency pulses, these hydrogen protons are dispersed and spin out of equilibrium straining against the pull of the magnetic field. As they relax back in to alignment with the magnetic field to their native state, they induce an electric charge, which it is measured and used for the image generation. Hence, the basic principle of the MRI technique is based on the measurement of these realignment rates, which are tissue dependent. 
Although MRI is mainly designed to provide structural information from the object of study, several techniques have been developed which permit the extrapolation of real time functional information, further expanding the applications of this modality [16]. Some of them are already established in the clinical practice such as functional MRI (fMRI), Magnetic Resonance Spectroscopy (MRS), Diffusion-Weighted Imaging (DWI) and molecular MRI (mMRI), while several more are currently being tested in the pre-clinical field, showing promising results especially in terms of sensitivity, where the aforementioned techniques are typically suffering from.

\subsubsection{Nuclear imaging}

Nuclear Imaging routine involves small amounts of radioactive tracers, also called radiopharmaceuticals [11][17]. These tracers are administrated into the patient, typically via intravenous injections, in order to localize and quantify possible existing pathologies. The tracers (probes), are made up of carrier molecules that are bonded to radioactive isotopes. The carrier molecule, as it was described before, interacts with specific proteins or sugar in the body while the isotope emits certain type of radiation. By detecting and measuring the emitted radiation, a precise determination of the location and accumulation of the tracer can be achieved [18]. The modalities that best represent the concept of nuclear imaging are: Gamma camera, SPECT and PET. A brief description of their operating principle is presented in the following:

\section{Gamma Camera}

Gamma cameras are the simplest imaging systems in nuclear medicine instrumentation, and they are employed to mainly carry out scintigraphy studies. In this technique, single gamma-ray emitting tracers are used, being the most typical ${ }^{99 m} \mathrm{Tc}(140 \mathrm{keV})$. The emitted gamma-rays are sensed by detectors which normally cover the whole FOV of the area of interest in the patient body. Both dynamic and static planar images can be obtained using this type of systems [19]. A gamma camera typically contains the following components:

a) A high-Z material collimator, providing the origin of the incoming gamma-rays

b) A scintillation crystal generating optical photons when excited by high energy gamma-rays (luminescence effect)

c) A photodetector, that in response to the absorption of a number of the optical photons, produces an electric signal

d) A series of electronic circuitries, designed to process the generated signals

e) A computer processing unit for data treating and image generation 


\section{Single Photon Emission Computed Tomography}

Single Photon Emission Computed Tomography (SPECT), is an imaging modality based on conventional gamma cameras, with the main advantage capability of providing tomographic information. Very shortly, SPECT systems typically integrate 1 or 2 gamma cameras that rotate around the object of study, carrying out multiple acquisitions at different views (the angular step varies from 3 to 6 degrees) [20]. Then, following a software reconstruction process, the tomographic images can be obtained.

\section{Positron Emission Tomography}

Positron Emission Tomography (PET) is another quantitative tomographic imaging technique [21] based on the coincidence detection of pairs of $511 \mathrm{keV}$ gamma-rays produced as the result of the annihilation of a positron with an electron. Positron emitters are combined with other molecules (radiopharmaceutical or probes) and injected into the patient.

The $511 \mathrm{keV}$ gamma-rays are detected using gamma detectors, also similar to the ones described in gamma cameras, which main difference is the absence of mechanical collimation, leading to a significant increase of sensitivity. The configuration of a PET scanner typically follows a 3D ring geometry, in order to maximize the angular coverage of the emitted gammarays. A coincidence window in the range of 5-10 nanoseconds is imposed in between the two detectors that measure the $511 \mathrm{keV}$ gamma-rays. Tomographic reconstruction processes are also then followed, in order to create cross-sectional 3D maps of the radiolabeled tracers accumulated in the object of study. More details of this modality are presented in chapter 2.

\subsubsection{Hybrid (Multimodal) imaging}

As said before, Molecular Imaging provides physicians a key diagnostic tool to access unique information regarding the diagnosis and treatment of many diseases. However, the unparalleled structural detail, lavishly provided by anatomical modalities such as Computed Tomography (CT) or MRI, still plays an important role in order to properly interpret molecular imaging information. Co-registration was a technique often used in order to overcome this kind of constrain [22][23]. However, the approach of using two modalities and separate acquisitions, exhibits several drawbacks from both clinical and technical points of view. For instance, the need for multiple scans of the patient (workflow), the patient positioning uncertainties and different system geometries, are a few examples. The need towards the fusion and possibly the simultaneous acquisition of the two-medical imaging modalities (anatomical and functional) into one physical unit, was profound [24].

In early 1990s, the concept of a hybrid PET/CT system was proposed by Townsend, Nutt and co-workers. In 1998, the first hybrid PET/CT prototype was built by CTI PET Systems (currently 
Siemens Healthcare). Since 2001, PET/CT scanners serve the clinical routine, consisting the most successful example of hybrid imaging (see Figure 1.6). Typically, the advantage of this concept is on the side of PET, as CT is excellent in providing anatomic detail to better correlate tracer uptake, as well as it provides great information for attenuation and scatter correction of the PET data. Today, hardly are seen standalone whole-body PET scanners in the clinical practice, as they have been replaced by the hybrid PET/CT systems, of course due to the aforementioned advantages [25]. In the same direction, in 2004 the first hybrid SPECT/CT was developed and commercialized by Siemens Medical Solutions. The use of SPECT/CT for a high number of applications has been widely extended due to the easier access to SPECT radiotracers and the lower cost of the overall system [26].

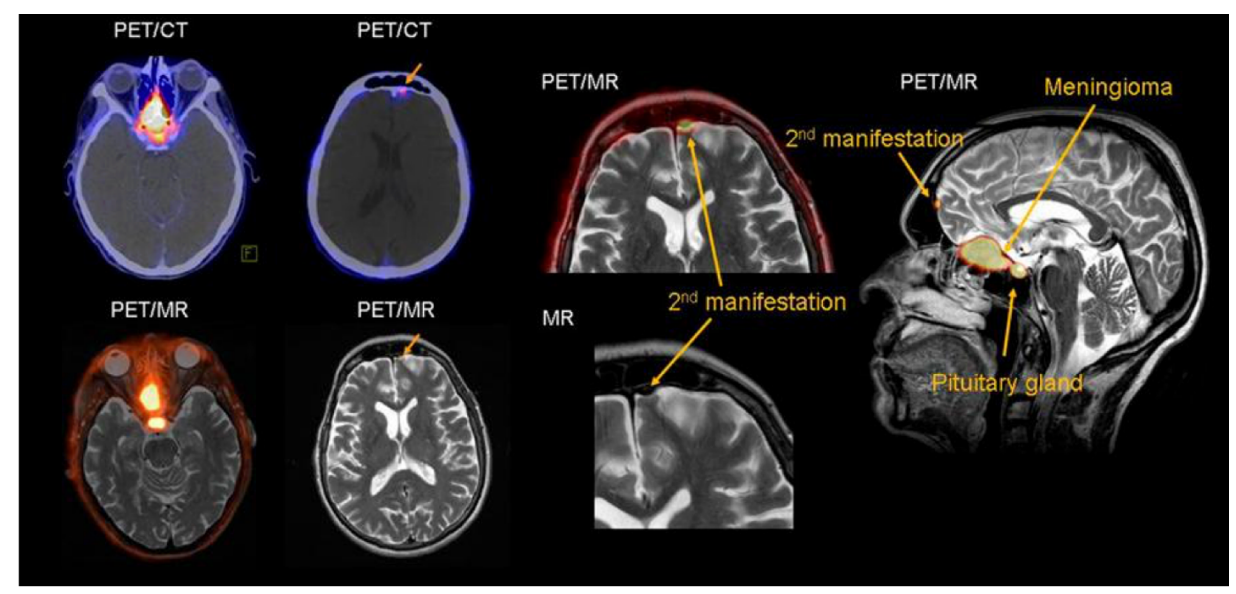

Figure 1.6. Brain images obtained with hybrid molecular imaging scanners and, in particular, with PET-CT and PET-MR. Extracted from [27].

In view of the success of PET/CT and SPECT/CT concepts, the scientific community was very attracted by the hybrid imaging approach of PET/MRI, potentially the most potential one for numerous applications (Figure 1.6) [28][29]. In contrast to PET/CT and SPECT/CT, PET/MRI (also SPECT/MRI) avoids the use of ionizing radiation to the patient when obtaining anatomical information. Moreover, MRI shows better image contrast for soft tissue than CT. Although efforts had initiated years before to merge PET and MRI technologies, a series of technical challenges related with the interferences of the magnetic fields to the PET detectors and vice-versa, made this kind of development extremely challenging. Another advantage of combining PET or SPECT with MRI, is the possibility to simultaneously acquire both imaging modalities, unlike PET/CT or SPECT/CT. In 2010 the first PET/MR systems were installed by Philips Healthcare (Ingenuity TF PET/MR [30]) following a tandem (sequential) approach since the PET design was based on Photomultiplier technology. Just few years later Siemens launched the PET/MR Biograph mMR [31], with the PET instrumentation based on Avalanche Photodiodes making it possible the simultaneous acquisition of PET and MRI images. These systems, enabled a novel and efficient diagnostic method in the clinical practice due to the fact that they combine the inherent advantages of MRI with the unique type of information that PET is capable of providing [28]. 


\section{References}

[1] D. J. Lomas and A. K. Dixon, 100 years and running: the medical imaging revolution. The Lancet. 346 (1995), Suppl:s20.

[2] B. S. Worthington, OBJECTIVES OF MEDICAL IMAGING. IEE Proceedings A: Physical Science. Measurement and Instrumentation. Management and Education. Reviews. 134 (1987), 98-100.

[3] H. Kasban, E. Atomic, E. Authority, A Comparative Study of Medical Imaging Techniques. International Journal of Latest Trends in Engineering and Technology. 4 (2015), 37-58.

[4] D. Schuijf, J. J. Bax, E. E. Van Der Wall, Anatomical and functional imaging techniques: Basically similar or fundamentally different?. Netherlands Heart Journal. 15 (2007), 4344.

[5] M. A. Pysz, S. S. Gambhir, J. K. Willman, Molecular imaging: current status and emerging strategies. Clinical Radiology. 65 (2010), 500-516.

[6] K. Zukotynski et al., PET/CT of dementia. American Journal of Roentgenology. 211 (2018), 246-259.

[7] D. A. Mankoff, A definition of molecular imaging. Journal of nuclear medicine. 48 (2007), $18 \mathrm{~N}-21 \mathrm{~N}$.

[8] B. A. W. Hoeben et al., 18F-FLT PET during radiotherapy or chemoradiotherapy in head and neck squamous cell carcinoma is an early predictor of outcome. Journal of Nuclear Medicine. 54 (2013), 532-540.

[9] L. Mosconi and P. F. McHugh, FDG- and amyloid-PET in Alzheimer's disease: is the whole greater than the sum of the parts?, Q. J. Nucl. Med. Mol. Imaging. 55:3 (2011), $250-64$.

[10] M. L. James and S. S. Gambhir, A Molecular Imaging Primer: Modalities, Imaging Agents, and Applications. Gambhir Physiological Reviews. 92:2 (2012), 897-965.

[11] F. D. Rollo, Molecular imaging: an overview and clinical applications. Radiology management. 25 (2003), 28-32.

[12] K. Chen and X. Chen, Design and Development of Molecular Imaging Probe. Current Topics in Medicinal Chemistry. 10 (2010), 1227-1236.

[13] R. W. Coatney, Ultrasound imaging: Principles and applications in rodent research, ILAR Journal. 42 (2001), 233-247.

[14] K. Shah and R. Weissleder, Molecular optical imaging: Applications leading to the development of present day therapeutics. NeuroRx. 2 (2005), 215-225.

[15] J. P. Hornak, The Basics of MRI. Biomedical Engineering. 24 (2008), 2-6. 
[16] A. Hengerer and J. Grimm, Molecular magnetic resonance imaging. Biomedical Imaging and Intervention Journal. 2 (2006), e8.

[17] F. G. Blankenberg and H. W. Strauss, Nuclear medicine applications in molecular imaging. Journal of Magnetic Resonance Imaging. 16 (2002), 352-361.

[18] A. W. Glaudemans et al., Nuclear medicine and radiologic imaging in sports injuries. Nuclear medicine and radiologic imaging in sports injuries. Springer Berlin Heidelberg, (2015).

[19] H. O. Anger, Scintillation camera with multichannel collimators. Journal of Nuclear Medicine. 5 (1964), 515-531.

[20] H. Wieczorek and A. Goedicke, Analytical model for SPECT detector concepts. IEEE Transactions on Nuclear Science. 53 (2006), 1102-1112.

[21] A. Shukla and U. Kumar, Positron emission tomography: An overview. Journal of Medical Physics. 31 (2006), 13-21.

[22] R. J. Hicks, E. W. F. Lau, D. S. Binns, Hybrid imaging is the future of molecular imaging. Biomedical Imaging and Intervention Journal. 3 (2007), e49.

[23] J. S. Lee and J. H. Kim, Recent advances in hybrid molecular imaging systems. Seminars in Musculoskeletal Radiology. 18 (2014), 103-122.

[24] B. J. Pichler, M. S. Judenhofer, C. Pfannenberg, Multimodal imaging approaches: PET/CT and PET/MRI. Handbook of Experimental Pharmacology. 185 (2008), 109132.

[25] T. Beyer et al., The future of hybrid imaging — part 2: PET/CT. Insights into Imaging. 2 (2011), 225-234.

[26] T. Beyer et al., The future of hybrid imaging — part 1: hybrid imaging technologies and SPECT/CT. Insights into Imaging. 2 (2011), 161-169.

[27] B. K. Das, Comparison of PET Ct with Pet MRI. Springer, New Delhi (2015).

[28] E. C. Ehman et al., PET/MRI: Where might it replace PET/CT? Journal of Magnetic Resonance Imaging. 46 (2017), 1247-1262.

[29] P. J. Maniawski, PET/MR: The molecular imaging dream team. Nuclear Medicine Review. 14 (2011), 47-50.

[30] H. Zaidi et al., Design and performance evaluation of a whole-body Ingenuity TF PETMRI system. Physics in Medicine and Biology. 56 (2011), 3091-3106.

[31] G. Delso et al., Performance measurements of the siemens mMR integrated whole-body PET/MR scanner. Journal of Nuclear Medicine. 52 (2011), 1914-1922. 


\section{Chapter 2}

\section{PET Fundamentals}

In this chapter we provide the key insights of PET, while strong emphasis will be given to the detectors themselves, describing each component along with its operating principle. Moreover, the PET characteristics that are critical for the quality of studies will be introduced and discussed. Nevertheless, and before anything else, a flashback in the first age of PET is reported, in order to better appreciate all steps made up to today.

\subsection{History of Positron Emission Tomography}

Positron Emission Tomography is on stage for more than 65 years, playing a key role over the whole panorama of Molecular Imaging [1]. PET has evolved following a series of advances in a plethora of research fields such as physics, chemistry, biology or computer science. Briefly, the timeline of how independent discoveries and developments led to the very firsts PET scanners, is presented below, split in four phases, as extrapolated from [2] and [3].

During phase one (late 1920 - late 1940), Paul Dirac published a paper entitled "A Theory of Electrons and Protons", in which he predicted the existence of the positron particle [4]. A few years later, Carl Anderson experimentally confirmed the existence of the positron, validating Dirac predictions, who was later awarded with the Nobel Prize in 1936 [5]. In the very same phase, the discovery of artificial radiation by Irene Curie and Frederick Joliot was made [6]. This great discovery, motivated investigators at the University of California in Berkeley to use their recently developed cyclotron to produce artificial radionuclides (positron emitters) such as ${ }^{11} \mathrm{C}$, ${ }^{13} \mathrm{~N}$ and ${ }^{18} \mathrm{~F}$ [7]. Lastly, two more important discoveries were made. First, Heinz Kallman, in 1948 proposed a new detector type capable of detecting $511 \mathrm{keV}$ photons, the so-called photomultiplier tube. Shortly after, and in 1949, Cassen, Curtis and Reed presented the calcium tungstate as a detector for high energy gamma photons [8].

In the second period (mid-1950s and the early 1960s), the first cyclotrons oriented for production of radionuclides for in vivo studies were installed in Washington University Medical Center and shortly after in Hammersmith Hospital in London [9]. Several more installations were followed; a fact that demonstrates the high interest of the researches and physicians in the medical application of the artificial radiation. In the same period, the scientific community was attracted by the inherent advantages of the coincidence detection, resulted from the positron-electron 
annihilation event, over single photon imaging. In particular, and almost concurrently Wren, Good and Handler from Duke university and Brownell and Sweet from Massachusetts General Hospital were working on this, novel for that moment approach, with the second ones to present the first type of positron imaging system, orientated for brain imaging and tumor identification [10][11] (Figure 2.1). Several more efforts were made in the following years to improve these concepts, but it was not until the starting of the third phase and the mid-1970 when the first PET systems were developed.

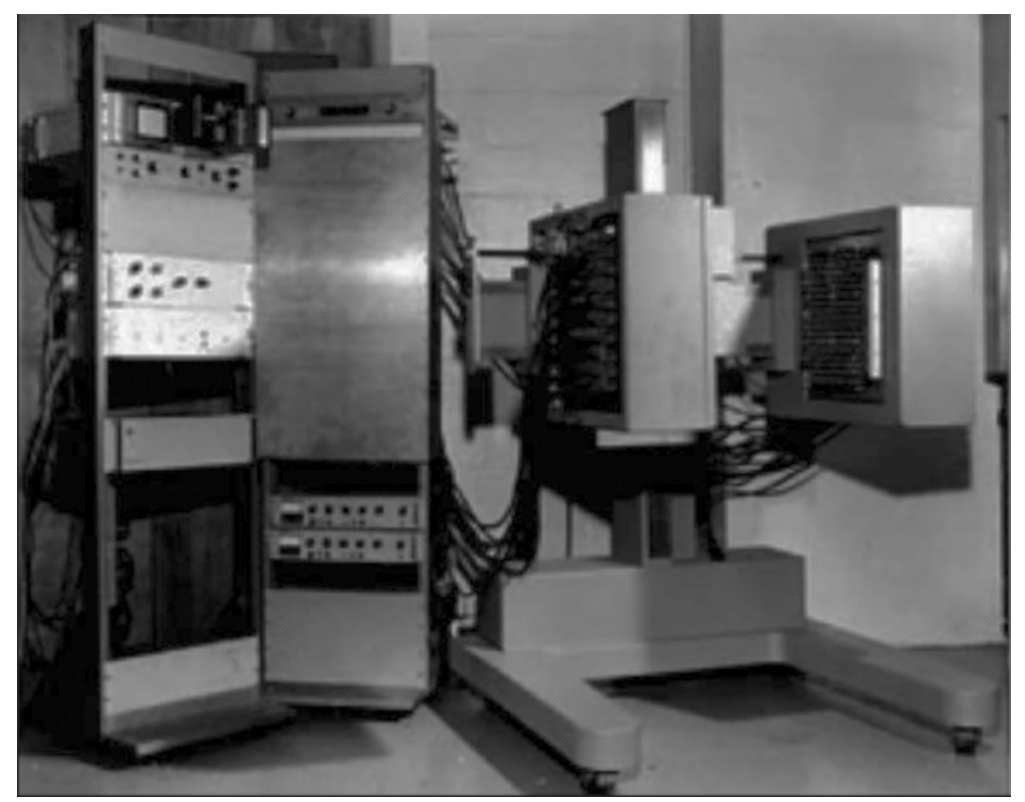

Figure 2.1. Photograph of the positron camera developed at the Massachusetts General Hospital in Boston [3].

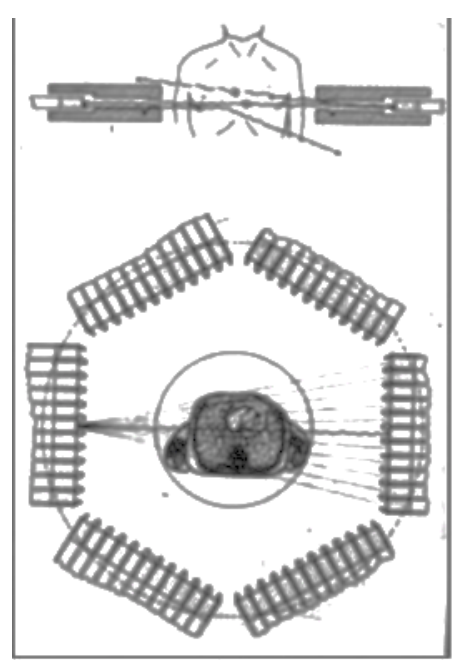

(a)

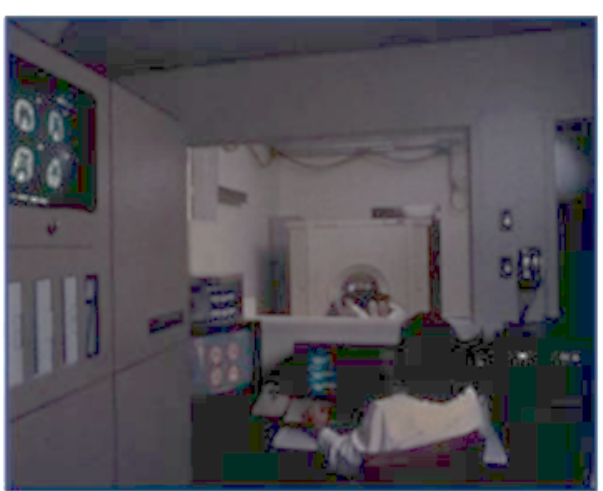

(b)

Figure 2.2. Left: A schematic of the ECAT II scanner developed by EG \& G ORTEC, which design was based on the PETT III developed by Ter-Pogossian and coworker. Right: ECAT I system view from the control room during a patient scan. Extracted from [3]. 
The third period (mid 1970s to 2000s), can be characterized by the great advances both in instrumentation and in radiochemistry. During this period, the first PET scanners in the form seen today, appeared. One of the very first successful approaches was the scanner built at Washington University between 1970 and 1973 by Phelps, Hoffman, Mullani and Ter-Pogossian named PETT2 [12]. This particular scanner was composed by a hexagonal array of $24 \mathrm{NaI}$ ( $\mathrm{Tl}$ ) detectors, and in 1975 was upgraded to fit clinical needs [13]. The upgraded version, named PET 3, was a whole-body scanner composed by $48 \mathrm{Nal}(\mathrm{Tl})$ detectors placed in a hexagonal array. Shortly after this development, Phelps and Hoffman collaborated with EG and G ORTEC to commercialize a single transaxial plane PET scanner branded as Emission Computerized Axial Tomograph or ECAT I (Figure 2.2 right). This system was later upgraded to ECAT II (Figure 2.2 left) [14], and its convincing performance led to its distribution in various centers during the following years.

Since 2000s, and after these three periods related to pure PET instrumentation, one can consider another period characterized by the introduction of multimodal imaging. In particular, the hybrid approach of PET/CT and its inherent advantages, created a new paradigm in clinical practice, resulting in the wide establishment and spread of PET technique (see again section 1.2.2.5).
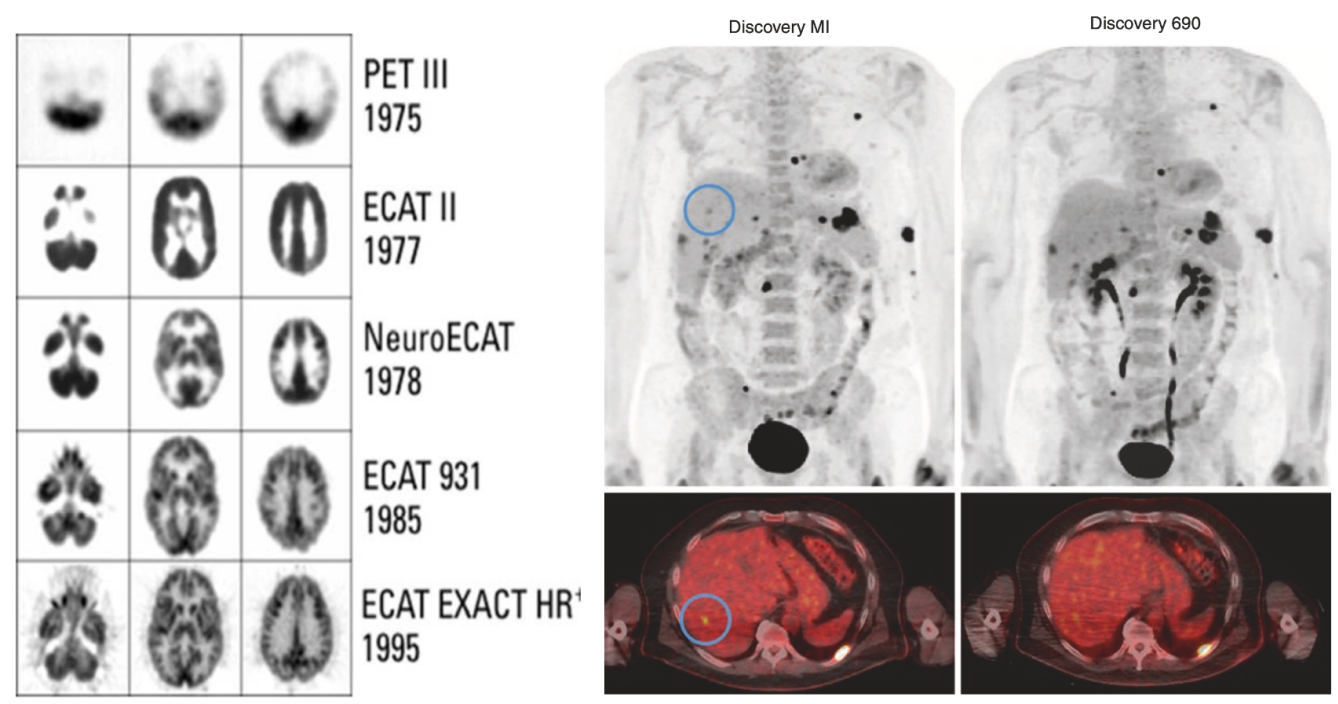

Figure 2.3. Left: Brain images obtained with early PET designs (from 1975 up to 1995) [15]. Right: Comparative clinical images from a patient with a clinical history of melanoma on, first, the D-690 (developed at 2011) and then the D-MI system (developed at 2016) (without time adjustment for activity decay). Top row shows MIP images; bottom row shows transaxial fused slice images [16].

These very first novel PET designs inspired and motivated a series of new developments. The progress recorded over the years in all aspects of PET is noteworthy (Figure 2.3) [1]. Nowadays, numerous systems capable of offering high quality images have been introduced in the clinical practice. For instance, some characteristic developments are described in [17]-[20]. Additionally, scanners have become more accessible, due to the huge efforts devoted on reducing system cost and complexity. Meanwhile, new scanner configurations, as we will discuss later, have showed 
significant benefits and have created new application areas [20]-[24]. Although PET imaging has been well enhanced over the last years, still exists some room for improvement.

\subsection{PET basics}

As it was briefly discussed in the previous chapter, all MI modalities are based on the use of molecular probes. Besides the fact that these probes carry the targeting moiety, thus the substance that will interact with the physiological procedure of interest, they also contain the tracer which will permit the detection of the probe by a molecular imaging scanner [25][26]. In the case of PET, the applicable tracer is always a positron emitting isotope. The isotope, due to the higher contained number of protons than neutrons, decays through positron emission ( $\beta^{+}$decay) [27]. The positrons are released with a known kinetic energy and thus, can travel through body tissues and organs. Meanwhile, they interact via coulomb interactions or bremsstrahlung with the surroundings electrons, upon reaching to a thermal equilibrium [28]. At this point, the positron due to its matter (anti-electron), annihilates with surrounding electrons resulting in the generation of two anti-parallel gamma-rays (see Figure 2.4). Notice that since the positron is not at-rest (initial momentum), this leads to not exactly anti-parallel gamma-rays. Each of these gammarays, is emitted with an energy of $511 \mathrm{keV}$, preserving the energy conservation principle [28][29].

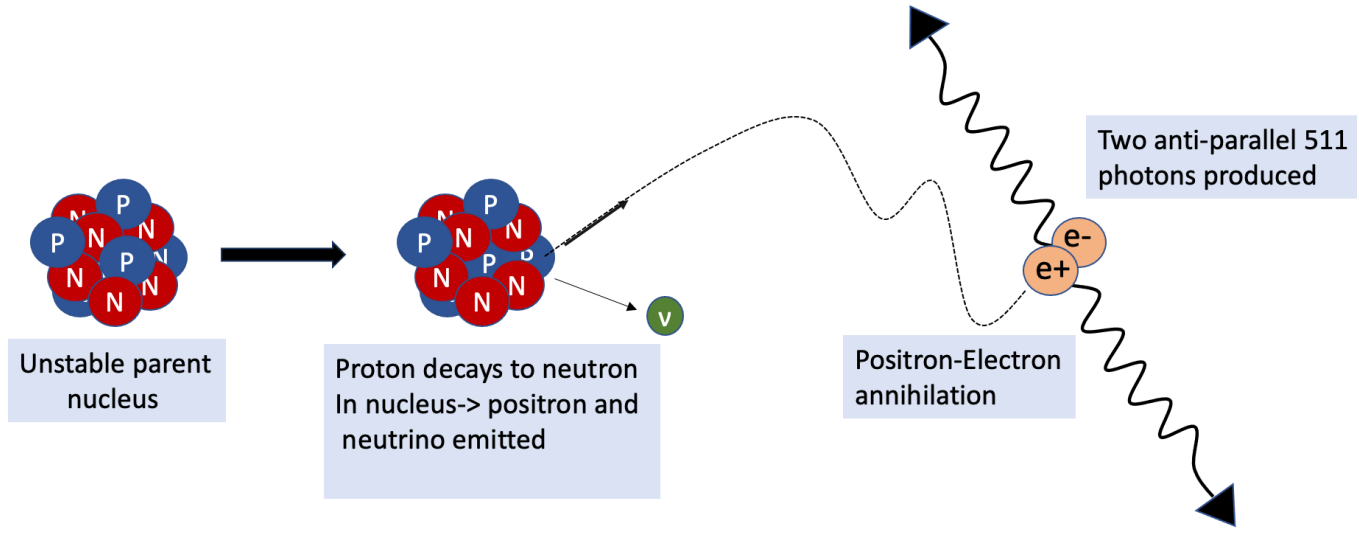

Figure 2.4. Positron-electron annihilation resulting in the generation of 2 back-to-back gamma-rays of $511 \mathrm{keV}$ each.

Several radionuclides have shown to be suitable for PET imaging, while some of them are preferred over others depending on the clinical applications [30][31]. Few of the most commonly used in PET applications are described in Table 2.1 along with their positron emission energies, half-life and mean range in water, being the last one the positron expected pathway upon reaching the thermal equilibrium [28]. Indeed, such mean range limits the image spatial resolution in a PET system.

To date, ${ }^{18}$ F-fluoro-deoxy-D-glucose $\left({ }^{18} \mathrm{~F}\right.$-FDG) is the most successful PET probe and it is highly used in PET practice as it follows a metabolic pathway, similar to glucose in-vivo. Another 
interesting molecule is a PSMA (prostate specific membrane antigen) tracer, which has been successfully linked to gallium-68 (proposed for specific prostate PET imaging).

\begin{tabular}{|c|c|c|c|c|}
\hline Isotope & Half-life & $\begin{array}{c}\text { Maximum } \mathrm{e}^{+} \\
\text {Energy }(\mathrm{MeV})\end{array}$ & $\begin{array}{c}\text { Mean } \mathrm{e}^{+} \text {Energy } \\
(\mathrm{MeV})\end{array}$ & $\begin{array}{c}\text { Mean } \mathrm{e}^{+} \text {range in } \\
\mathrm{H} 20(\mathrm{~mm})\end{array}$ \\
\hline${ }^{18} \mathrm{~F}$ & $109.77 \mathrm{~min}$ & 0.64 & 0.25 & 0.6 \\
\hline${ }^{11} \mathrm{C}$ & $20.4 \mathrm{~min}$ & 0.960 & 0.386 & 1.2 \\
\hline${ }^{68} \mathrm{Ga}$ & $67.6 \mathrm{~min}$ & 1.89 & 0.89 & 2.9 \\
\hline${ }^{15} \mathrm{O}$ & $122 \mathrm{sec}$ & 1.372 & 0.735 & 3.0 \\
\hline${ }^{64} \mathrm{Cu}$ & 12.7 hours & 0.653 & 0.278 & 0.7 \\
\hline${ }^{89} \mathrm{Zr}$ & 78.4 hours & 0.902 & 0.396 & 1.3 \\
\hline${ }^{22} \mathrm{Na}$ & 2.6018 years & 0.55 & 0.22 & 0.5 \\
\hline
\end{tabular}

Table 2.1 Most relevant radionuclides along with their main characteristics exploited in PET [31].

\subsection{PET detectors}

In order to detect the aforementioned gamma-rays and decode with accuracy the point-ofinteraction, gamma-ray detectors are employed [32]. These are the key instrumentation elements of every PET scanner. Typically, in PET, the detectors are placed in a ring configuration surrounding the object of study and, thus, to favor the detection of the isotropically generated pairs of $511 \mathrm{keV}$ gamma-rays (see Figure 2.5 right).
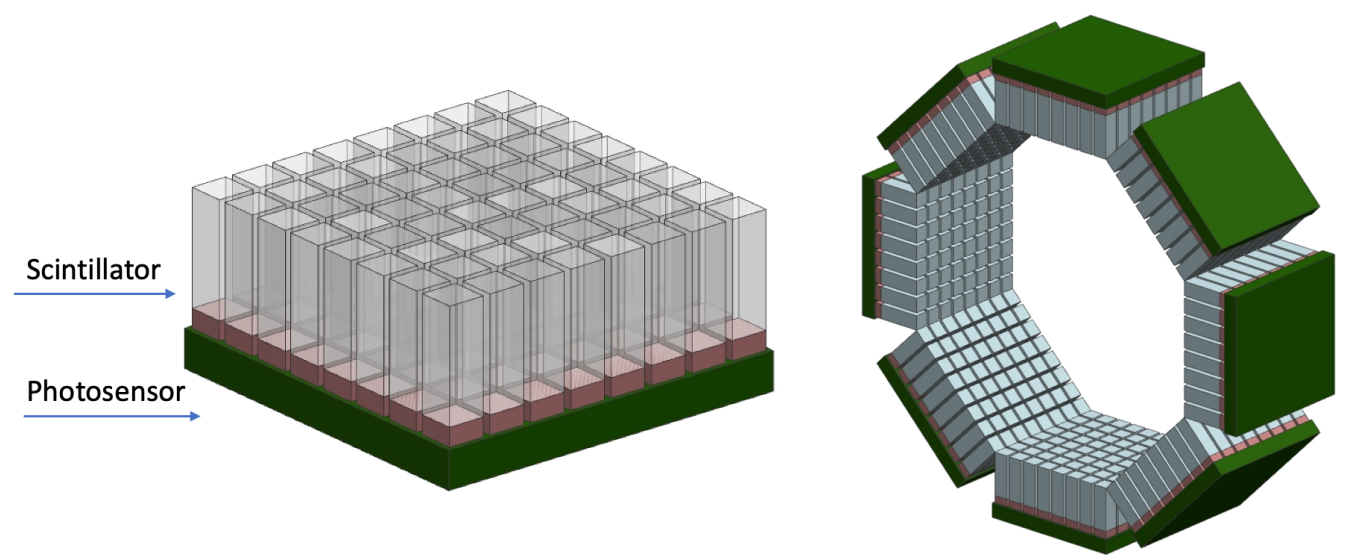

Figure 2.5. Left: PET detector block illustration, composed by a scintillator in a form of crystals array and an $8 \times 8$ SiPM photosensor array. Right: Representation of a PET ring configuration, composed by 8 detector blocks.

In general, each PET detector is composed by a high-density scintillation material coupled to a photodetector (Figure 2.5 left). Although different types and approaches of each component exist nowadays, their operating principle and purpose are quite similar. In the following sections, a comprehensive description of each of these elements is presented. 


\subsubsection{Scintillators and $\gamma$-ray detection}

Scintillators are materials which emit electromagnetic radiation in the visible or ultraviolet range of spectrum when excited by ionizing radiation [33]. This light is isotopically emitted and proportional to the amount of energy deposited by the gamma-ray.

\subsubsection{Interaction with matter}

When a high energy gamma-ray reaches a scintillation material, it may interact with it following three ways (Figure 2.6) namely photoelectric interaction, Compton scattering and pair production [27][28]. In PET applications, the situation to be favored is the photoelectric effect, due to the fact that this enables the detection of the whole amount of energy of the incident ray in a single interaction.
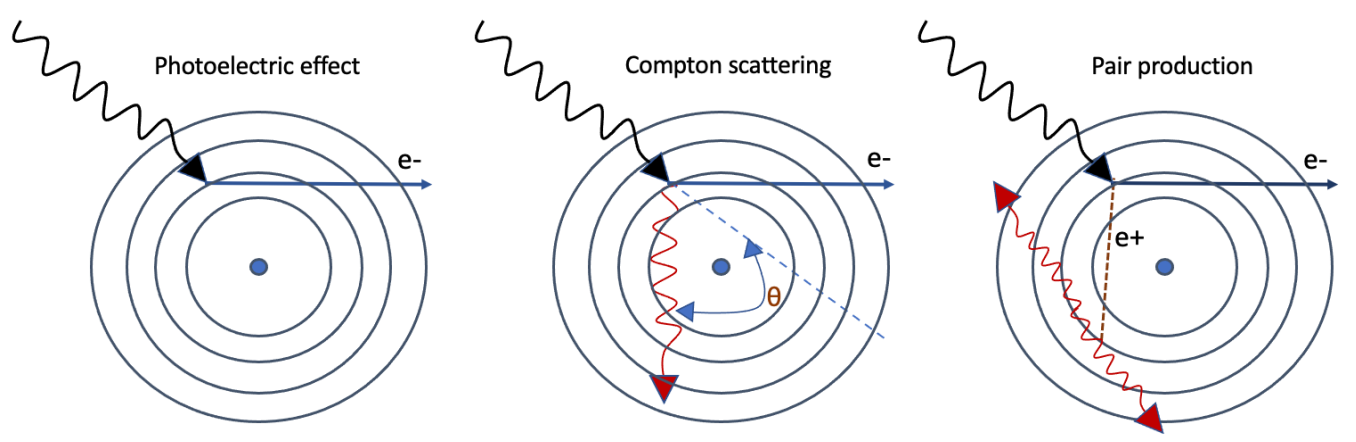

Figure 2.6. Interaction of a gamma-ray within an atom. Left, photoelectric effect; Center, Compton scattering; Right, pair production.

For that reason and in order to stimulate these photoelectric interactions, scintillators with high atomic number and high photoelectric cross-section are preferred [32]. Lastly, the interaction based on pair production, is not encountered in medical applications, since it can only occur if the incident photon has at least $1.022 \mathrm{MeV}$ energy [27][32].

\subsubsection{Scintillation mechanism}

In the scintillators of interest in PET, when a Compton or photoelectric interaction occurs, electrons are excited from the valence band leaving an associated hole behind [27]. The electrons are promoted to either the conduction or the exciton band, later they relax to this band, with the consequent photon emission. In order to increase the number of emitted scintillation photons, small amounts of impurities, called activators, are added to the crystal. These impurities create electronic levels in the forbidden gap, without modifying the energy structure of the overall crystal (see Figure 2.7). Through this process, and since the distance of the levels is in the order of a few electronvolts, electrons can de-energize emitting photons that lie in the visible or near ultraviolet range [33][34]. 


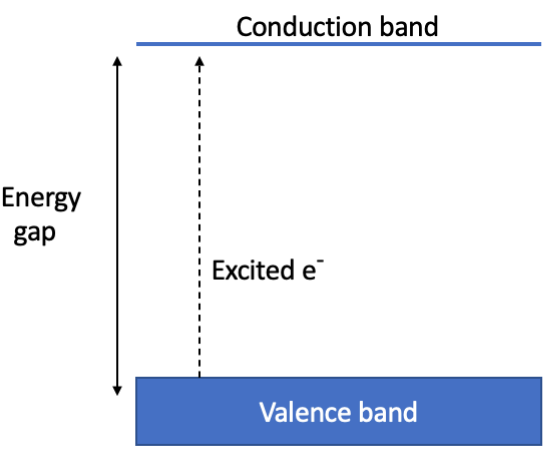

(a)

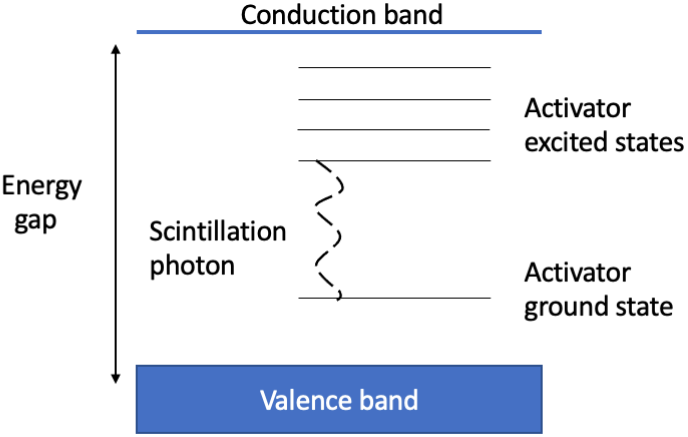

(b)

Figure 2.7. Energy band structure of an inorganic scintillator: (a) pure scintillator, (b) activated scintillator.

\subsubsection{Scintillator types}

A large variety of scintillator types exists [35][36]. They are mainly categorized into organics and inorganics. Inorganic crystals are somehow preferred for PET applications due to their higher atomic number as described above. Additionally, the scintillators can be found in form of solids, liquids or even gases, with the solid ones to be, by far, the choice of preference in PET [37].

Another, highly important aspect of the scintillators is their geometrical configuration. Solid scintillators are mainly seen in two configurations; in form of crystal arrays (pixelated crystals) and as monolithic crystals [28][35][37][38]. Pixelated crystals are matrices of individual crystal elements typically optically isolated by reflective materials, meaning that most of the generated scintillation photons are optically confined inside a given crystal element. This type of crystals is highly exploited in PET and used in most of clinical installed systems. Regarding monolithic crystals, these are continuous blocks, which nowadays have attracted the interest both of the academic and industrial communities due to their inherent advantages in terms of spatial resolution. Since, there is no gap occupied by reflector materials, the generated optical photons freely travel inside the block, until reaching the photosensors, creating a light distribution relatively easily decodable. Additionally, this lack of any type of gap, results in high packing fraction. In Figure 2.8 both scintillator configurations are represented.

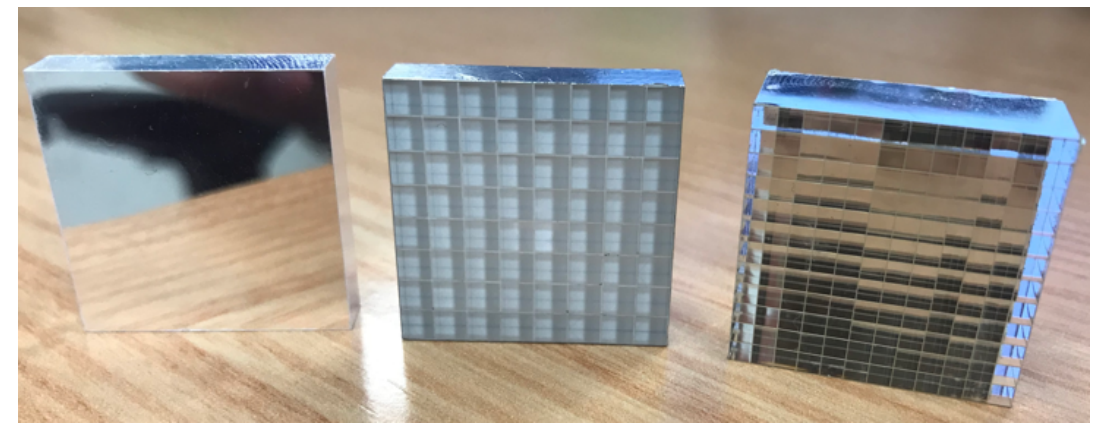

Figure 2.8. Examples of scintillation crystals. From left to right, monolithic crystal $25 \times 25 \times 5 \mathrm{~mm}^{3}, 8 \times 8$ elements crystal array $\left(3 \times 3 \times 5 \mathrm{~mm}^{3}\right)$ and crystal array of $16 \times 16$ elements $\left(1.5 \times 1.5 \times 10 \mathrm{~mm}^{3}\right)$. 
Generally speaking, scintillators geometrical configurations nowadays are fully customizable and can be typically adopted in all design concepts and needs.

Regarding the suitability of each scintillator itself, this strongly depends on the system configuration and requirements, however, some major factors exist and need to be considered when it comes to characterize the appropriateness of a scintillator. The key factors can be summarized as the following ones [32][39]:

- High Light Yield (LY), a factor related to the number of light photons generated as a function of the incoming photon energy

- High stopping power, the attenuation coefficient of the absorbed radiation, for a given material thickness

- Short rise and decay times, which corresponds to the time needed for a crystal to emit all optical photons for a given gamma-ray interaction

- Refraction index, a factor corresponding to how efficiently the generated optical photons can be transmitted to the photosensor

- Cost and accessibility

Despite the numerous types already proposed, only a small fraction of them is capable of combining, in a satisfying way, the desired features. Some of the scintillators more widely used are presented in the following table, along with their main characteristics [28][35][39][40].

\begin{tabular}{|c|c|c|c|c|c|c|c|}
\hline Crystal & $\begin{array}{c}\text { Light } \\
\text { yield } \\
(\mathrm{ph} / \mathrm{MeV})\end{array}$ & $\begin{array}{c}\text { Decay Constant } \\
(\mathrm{ns})\end{array}$ & $\begin{array}{c}\text { Max } \\
\text { Wavelength } \\
(\mathrm{nm})\end{array}$ & $\begin{array}{c}\text { Refractive } \\
\text { index }\end{array}$ & Hydroscopic & $\begin{array}{c}\text { Density } \\
\left(\mathrm{g} / \mathrm{cm}^{3}\right)\end{array}$ & $\mathrm{Z}_{\text {eff }}$ \\
\hline Nal & 45000 & 230 & 415 & 1.85 & Yes & 3.67 & 51 \\
\hline BGO & 8500 & 300 & 550 & 2.15 & No & 7.13 & 74 \\
\hline LSO & 33000 & 40 & 420 & 1.84 & No & 7.4 & 66 \\
\hline LYSO & 32000 & 42 & 420 & 1.86 & No & 7.2 & 63 \\
\hline LaBr $_{3}$ & 22000 & 16 & 360 & 1.8 & Yes & 5.8 & 46.9 \\
\hline GAGG & 57000 & 88 & 520 & 2.03 & No & 6.63 & 54.4 \\
\hline
\end{tabular}

Table 2.2. Properties of some of the scintillators typically seen in PET applications.

Currently, many efforts are being devoted on improving the status of the scintillation materials. These efforts are motivated from the well-defined need to develop brighter (higher light yield), faster and more efficient scintillators. For this purpose, doping approaches, new materials as well as the scintillation mechanism itself are constantly explored and evaluated. 


\subsubsection{Photodetection process}

As it was previously described, following the interaction of the gamma-ray inside the scintillator, optical photons are generated [32]. The optical photons typically have an energy of few electronvolts. These photons are converted into electrical signals through photon-sensitive devices, which are optically coupled to the scintillators. The amplitude of these electrical signals is directly proportional to the number of detected photons. Two main photosensor devices have been employed in PET applications: the so-called photomultiplier tubes and the silicon photomultipliers.

\subsubsection{Photomultiplier tubes}

In photomultiplier tubes (PMTs), the process starts when photons reach and excite a thin metal layer called photocathode, resulting in the generation of photoelectrons. These photoelectrons, are then accelerated towards a chain of dynodes, connected to a sequentially increase voltage. As soon as the photoelectrons strike the dynode, secondary low energy photoelectrons are then produced and accelerated towards the next dynode. This process is repeated several times, leading to the generation of a photoelectron cascade (Figure 2.9). After a few nanoseconds, the first photoelectron results in the generation of the order of $10^{6}$ electrons and, thus, into an electrical signal easily detectable by electronics [32][41][42].

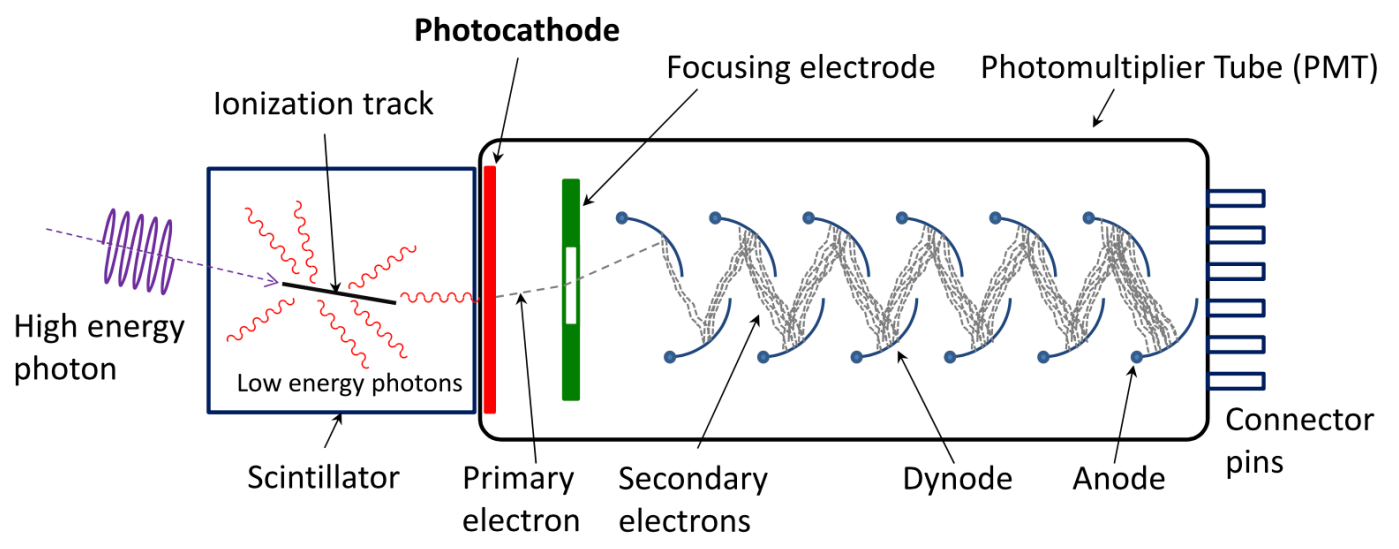

Figure 2.9. Schematic of a photomultiplier tube principle, showing the amplification process in the dynodes.

PMTs have been exploited in PET for long time. In fact, most of the still installed clinical PET systems worldwide are based on this type of photosensors. Their main advantages are stability, high gain that results in a good Signal-to-Noise Ratio (SNR) and fast signal response (rise time). However, mainly due to their bulkiness and sensitivity to magnetic fields, alternative photodetector devices have been proposed. 


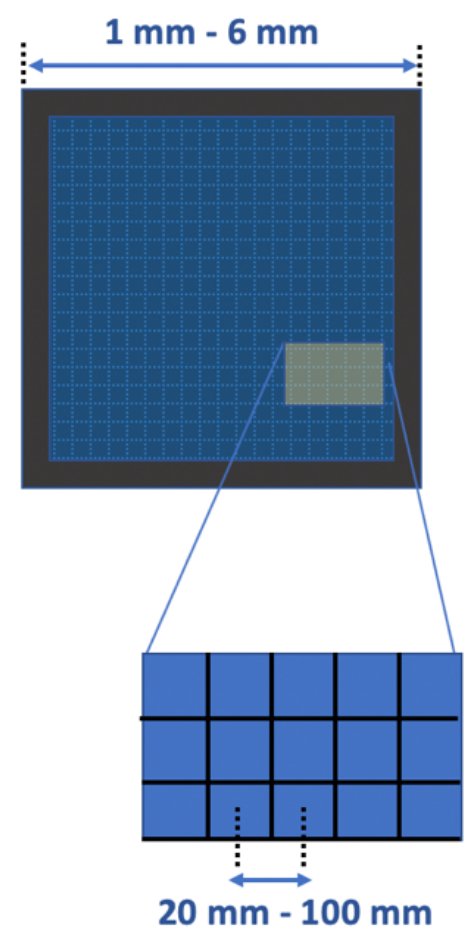

Figure 2.10. Sketch of a SiPM element along with its basic components; the SPADs.

\subsubsection{Silicon photomultipliers}

Silicon photomultipliers (SiPMs) are solid state photodetection devices which shortly after their appearance to the market, started to replace the well-established PMTs [43][46]. Essentially, SiPMs are composed by Single Photon Avalanche Diodes (SPADs), also known as cells or pixels (Figure 2.10) [47]. All cells are connected in parallel while each one contains its own quenching resistor. Each SPAD, in reality is a $\mathrm{p}-\mathrm{n}$ junction or diode, biased in reverse direction above the breakdown voltage. Once the bias is sufficiently high, a high field across the diode is created, which results in the generation of a large avalanche current, once the first photon is detected. Each SPAD is independent, and capable of detecting one photon. The estimation of the magnitude of the generated photon flux can be extrapolated with a certain precision, by the sum of all photocurrents of all SPADs, in a quasi-analog output [43].

Typically, the SPADs of each SiPM follow an array configuration, with the size of each SPAD to be in the order of some micrometers, varying depending on the type and manufacturer. The number of microcells contained in one SiPM is of high importance, as it directly reflects the total amount of photons that can simultaneously be detected [48]. Typically, the number of SPADs inside a SiPM varies from a few hundred, up to a few thousands. Hence, the total number and their individual size, results in the so-called fill factor. SiPM size also varies, with the most typical configurations to be squares with sizes varying from $1 \mathrm{~mm} \times 1 \mathrm{~mm}$ to $6 \mathrm{~mm} \times 6 \mathrm{~mm}$. The SiPM size is referred as their active area. Moreover, SiPMs are also found in arrays configurations in order to form realistic photodetectors in PET instrumentation (see Figure 2.11).

The compact size of SiPM, the low bias voltage required (25-100 V), the large availability of providers, their compatibility with magnetic fields and their fast response of the internal avalanche amplification, are some of their advantages. However, they also show some disadvantages. Factors such as amplitude and gain fluctuations, crosstalk between individual cells and correlated noise might limit their performance. In addition to these, SiPMs are sensitive to dark counts, due to the generation of thermal electrons which may initiate an avalanche photocurrent, leading to false triggering of electronics. Today, the large variety of SiPM types available, as well as the number of providers, directly reflects the interest of the community in these types of sensors. SiPMs are typically characterized by several parameters related with their performance and requirements [48][49]. Some of the most important ones are described below: 
1. Photodection efficiency (PDE): reveals the sensitivity of a SiPM to a photon detection based on the wavelength of the incident light, the applied overvoltage and the fill factor.

2. Gain of the SiPM: the amount of charge produced for each detected photon, directly related with the overvoltage value and the SiPM fill factor.

3. SPTR: Single Photon Time Resolution, reflects the time precision at which a SiPM is capable of detecting the time arrival of a single photon.

4. Dark Count Rate (DCR): avalanche provoked by a thermally generated electron and not by an incident photon. The DCR is related with the overvoltage and temperature.

5. Optical crosstalk: a factor related with the probability that an avalanching SPAD induces an avalanche in a second SPAD. This factor is related with the applied overvoltage as well as the SiPM fill factor.

6. Dead Time: the time required by each SPAD to be recovered for the detection of a new incoming photon.

7. Dynamic Range and Linearity: the dynamic range of a given SiPM sensor can be defined as the optical signal range over which the sensor provides a useful output.

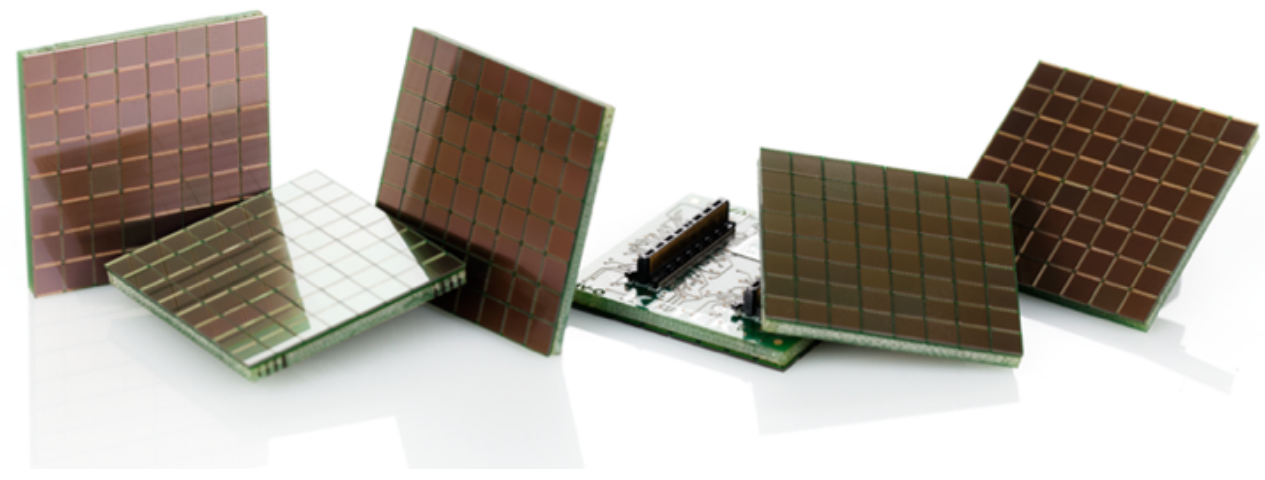

Figure 2.11. SiPM arrays $8 \times 8$ elements of $3 \times 3 \mathrm{~mm}^{2}$ active area (KETEK, PA3325). Extracted from [50].

\subsubsection{Digital SiPMs}

In accordance to what it was described earlier, SiPMs operate in an analog way. One of the main drawbacks of this operating principle is that all individual SPADs are read out by a common circuit, which this might result in noise and jitter generation. To overcome this limitation, a digital approach of the SiPM was suggested [51][52]. The digital SiPMs were proposed in 2009 and shortly after commercialized by Phillips Digital Photon Counting (PDPC). This type of sensors is based on SPADs integrated in a standard CMOS technology and consist a fully digital device. Each SPAD that experiences an avalanche breakdown process, generates its own digital output that is captured, along with the digital outputs from all other triggered microcells, by a photoncounting and time-stamping logic circuit. This all-digital concept, shows a series of advantages in photodection. Besides the fast and accurate photon counting, the time detection of the very first photon can be precisely decoded. Additionally, in these sensors, the recovery time of each SPAD is significantly lower compared to analog SiPMs due to an integrated transistor (recharged element) that quickly recharges the diode back to its sensitive state after breakdown. Lastly, 
digital SiPMs permit users and engineers to disable individual SPADs that are sensitive to dark counts and noise generation as they are also equipped with an addressable static memory.

Digital SiPM are a remarkable design concept that takes advantage of the already digital nature of the SPADs themselves. Hopefully, it will guide many efforts towards reaching to novel and more efficient SiPM structures, excluded from all sources of noise intrinsic to analog components.

\subsubsection{Readout electronics}

Each PET detector includes some type of electronic circuitries which serve for the signal treatment, generated by the photosensors following the scintillation event. They are typically referred as readout electronics, and are the key elements towards the extrapolation of the quantities of interest in a digital format [32]. Before proceeding to a more detailed description of the readout process, it is important to define these quantities of interest which are both critical for the decoding of each annihilation event. Therefore, all PET readout circuits should be capable of extrapolating:

1. The total charge produced by each photosensor following a scintillation event, corresponding to the total amount of photons detected.

2. The fraction of time in which the photodetection took place.

The output signals from the photosensors are typically analog with a duration in the order of few hundreds of nanoseconds (depending on the scintillator type) [28][29]. Figure 2.12 shows an example of one of these pulses. As it can be observed, these signals exhibit a relatively sharp rise

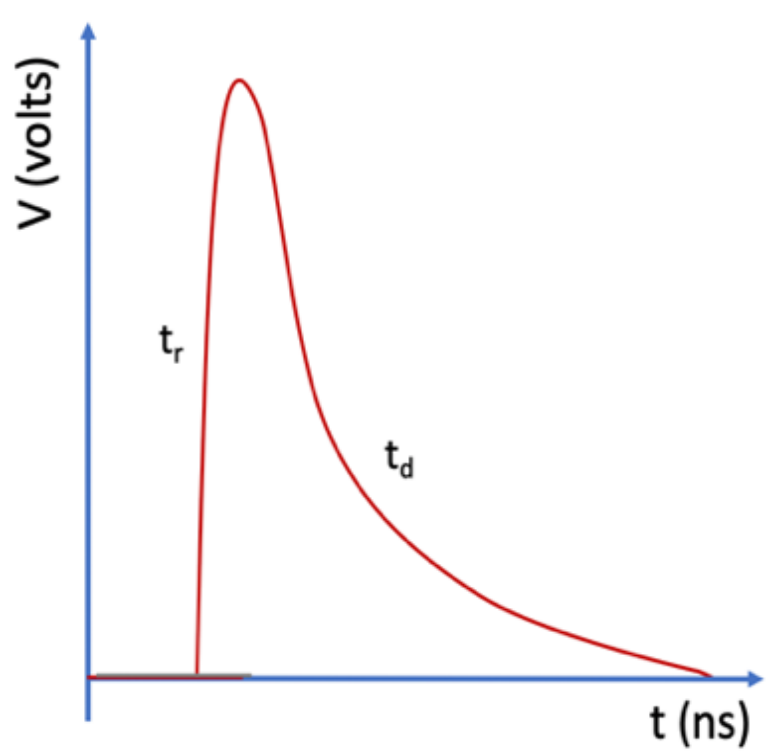

Figure 2.12. Representation of a gamma signal outputted by a SiPM and an inorganic scintillator. time $\left(\mathrm{t}_{\mathrm{r}}\right)$ in followed by an exponential decay curve $\left(t_{d}\right)$. The area contained within the signal provides information directly related with the charge collected by the photosensors. This information is typically obtained using Analog-ToDigital Converters (ADCs), which provide a digital value corresponding to that collected charge and, thus, proportional to the gamma-ray energy [28][32][53]. While digitizing pulses with ADCs is a well standardized method in radiation detectors, the Time-overThreshold (ToT) method has also been proposed and widely used [53][54]. This approach is based on a time width pulse 
processing that decodes the energy by measuring the pulse width [55]. In general, the energy information is easily accessible and can be decoded precisely [32][56]. However, regarding the timing information, its determination results somehow more challenging especially when high precision is aimed. Although this information is intrinsic in the sharp rising edge of the analog pulse $\left(t_{r}\right)$, in which time frame the time arrival of very first optical photons is reflected, for most conventional systems is quite challenging to decoded it. Therefore, conventional PET instrumentation, simply relies on rough timing estimations from the whole signal shape. However, due to the great improvements in microelectronics, nowadays several solutions or approaches exist, which permit the accurate decoding of a signal rising edge and thus, of the first photons time arrival [28].

As it will be further discussed later, an accurate determination of the signal time (gamma-ray impinging time) can significantly boost PET performance. However, reaching a good precision, demands a series of steps which in several cases are challenging. Methodologies, specific approaches and designs are required to be combined in the proper way, upon reaching to the desired results.

Several types of readouts have been proposed and used over the years in PET instrumentation. (see for instance Figure 2.13). The suitability of a readout design is linked to three interrelated main system factors: performance, cost and complexity. Typically, a compromise has to be made among these three factors, aiming to design and build PET detector blocks that meet high system performance, while keeping the cost and complexity at a reasonable level.
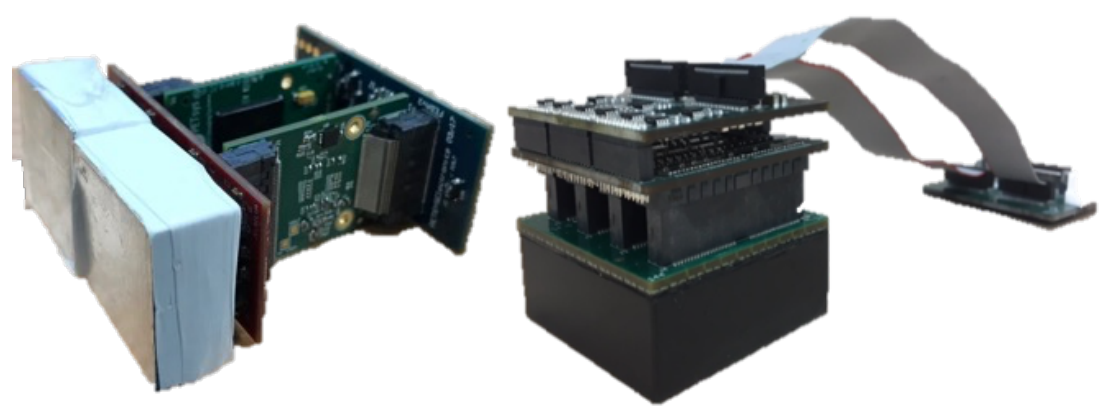

Figure 2.13. Examples of Readout-Electronics approaches, when these are coupled to SiPM photosensors and scintillators (of different size and type). Left: ASIC (TOFPET2, PETsys Electronics) based readout. Right: Analog multiplexing readout.

The most obvious way to read-out a multichannel sensor, is the processing of all independent photosensor elements. Although the advantages of this approach are numerous, these methods result in a significant number of signals to be characterized and processed, inferring a high development and system cost. A very interesting approach, highly exploited in the field of gamma-ray instrumentation, is the signal reduction or multiplexing. A large variety of readout architectures based on passive component network circuits have been proposed all over the years 
(Figure 2.14 right), which permit the combination of photosensor outputs through specific readout circuitries. Some representative examples can be found in the works [57][61]. These approaches eventually result in a significant reduction of the channels to be digitized and processed. Although these readout concepts serve for a variety of systems designs providing good results, they typically exhibit challenges when it comes to an accurate timing determination of the signals, due to possible time delays in their paths as well as due to mismatching of terminal capacitances. Apart from this, in several cases the rate capabilities might also be impacted due to the analog processing circuits. These limitations revealed the need for novel, faster and more efficient readout methods.
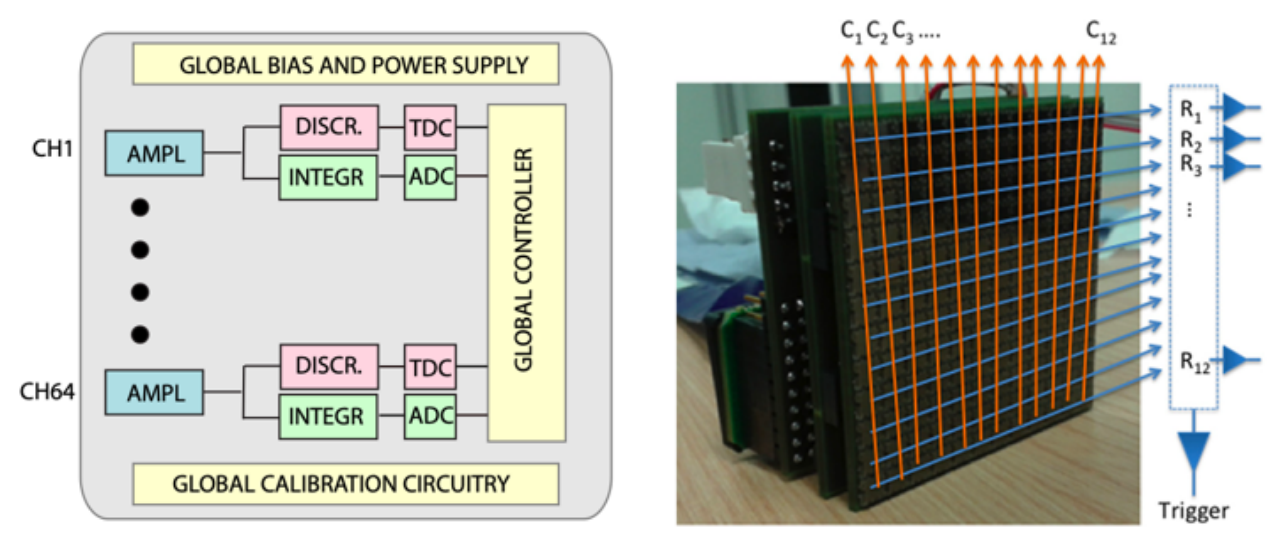

Figure 2.14. Left: Simplified block diagram of the TOFPET2 ASIC, designed to input directly up to 64 gamma signals from SiPMs. Right: Reduction scheme where the 144 signals resulting from a $12 \times 12$ SiPM array are reduced to just $12+12$.

Currently, one of the most promising approaches for the readout electronics in PET detectors are the so-called Application Specific Integrated Circuits (ASICs) [62]. Despite their small size, they enable the possibility to incorporate many custom circuitries. Both analog and digital components (e.g. ADCs, TDCs, etc.) can be integrated to develop complex readout designs, almost noise free, and with high performance capabilities. This technological achievement, in PET is translated as a good alternative for reading and processing a high number of signal channels, while cost and complexity are somehow conserved. Today, numerous ASICs designs, suitable for PET, have been developed and some industrialized [63][69]. In Figure 2.14 left, the readout scheme of a commercially available ASIC, extensively used during this $\mathrm{PhD}$ work, is shown. This specific chip can read up to 64 independent channels, while for each of them integrates an electronic amplifier (current conveyor) followed by two post amplifiers, one for the time and one for the energy decoding [63][64].

Nowadays, in PET designs both performance and cost are compromised. Therefore, ASICs are continuously gaining ground, substituting the established analog readout schemes. Many efforts are devoted on improving current ASIC designs and technological standards creating a quite promising future for a variety of PET applications. 


\subsection{Coincidence principle and PET reconstruction}

Since the annihilation event will result in the generation of two back-to-back gamma-rays, two opposed detectors need to sense these rays. This almost simultaneous detection, results in the so-called coincidence event. Then, the extracted quantities of interest described above, will permit the decoding of the point of interactions inside each detector volume. This information will be used to define a Line of Response (LOR), which simply indicates that the annihilation event has took place somewhere along this line. After the recording of many (typically millions) of such LORs, and following complex reconstruction algorithms, PET images can be generated [28].

Image reconstruction, is the last, but not least, process in PET technology. It involves the generation of cross-sectional images of the radiotracer distribution inside the object of study. The reconstruction process, as explained earlier, exploits the information originated from all LORs defined during the acquisition, in order to visualize the activity distribution of the radiotracer (Figure 2.15). The main methods to reconstruct these tomographic images can be grouped into analytical or iterative [70][71].

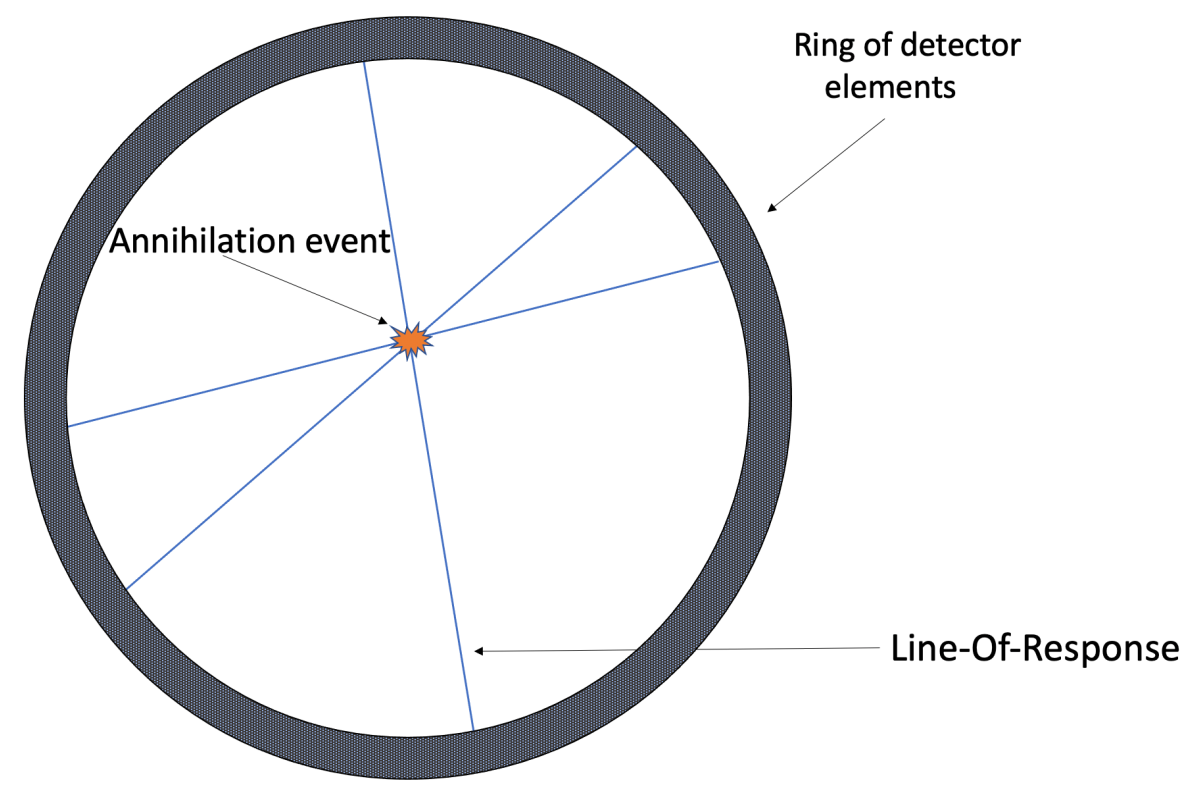

Figure 2.15. Hotspot localization through LORs intersection.

Analytical methods assume that a unique solution and noise-free exists. Thus, direct mathematical solutions are applied to generate an image. However, this assumption can infer challenges, since the influence by physical effects and system noise is usually significant. Typically, these methods result in low quality images, but serve to provide the true system resolution [72]. The most commonly analytical methods currently used are the Back ProjectionFiltering (BPF) and Filtered-Back Projection (FBP). 
Iterative reconstruction algorithms are somehow tougher to implement, but they return enhanced quality images. In this approach the possible noise contribution is accounted, meaning that a unique solution does not exist. Therefore, through realistic models and feedback loops, they provide the optimum possible solution. Iterative algorithms demand higher computing resources compared to analytical approaches. However, due to improvements in computational processes such as the usage of multiple CPU (Central Processor Units) or GPU (Graphical Processor Units), iterative methods are still the most extended at the clinical level. The iterative methods most frequently used are the Maximum Likelihood-Expectation Maximization (MLEM) [73] and the Ordered subsets Expectation Maximization (OSEM) [74], with the second one also to be exploited in clinical practice.

\subsection{PET specifications}

Each single improvement achieved in PET can be considered vital, as it typically leads to an increase in the value of the diagnostic information that can be extracted. Fortunately, nowadays PET technology has already seen significant progress, essentially benefiting the clinical and preclinical practices. This evolution might be graphically reflected in Figure 2.16, in which the PET imaging standards of across the years are depicted [75][78].
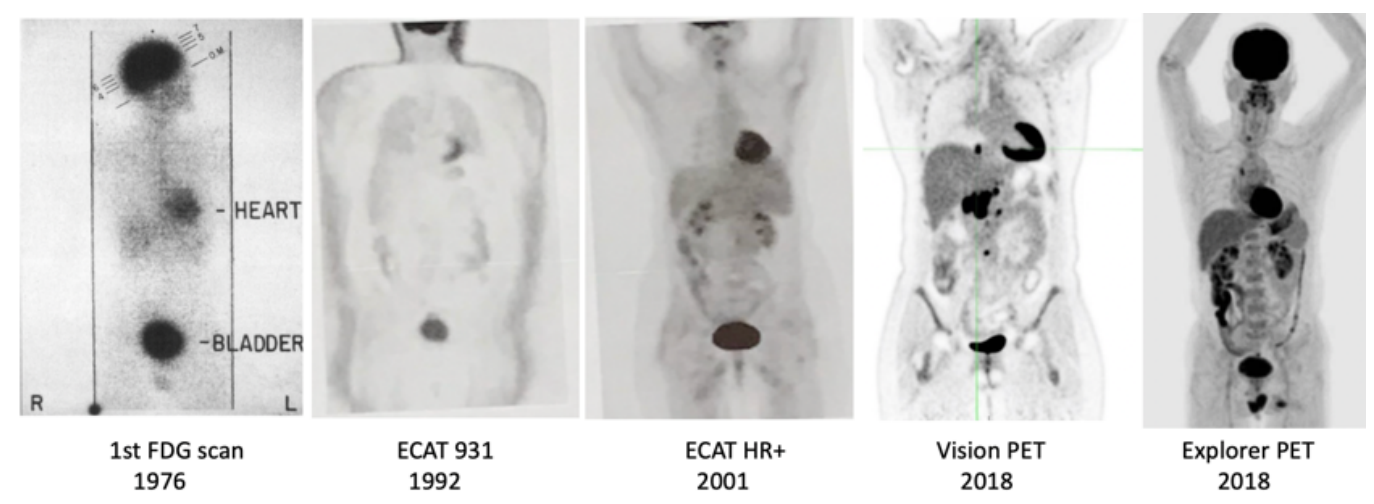

Figure 2.16. Evolution of PET imaging images over the years [3][76][77][79].

This evolution is a result of huge efforts devoted in developing novel PET configurations which meet specific requirements and specifications. Having already reviewed the background knowledge, now is adequate to discuss some of these specifications, which as it will be later shown, are directly related with scanners performance and images quality.

\subsubsection{Spatial resolution}

In Figure 2.16, someone might observe the significant improvement in the quality of the generated PET images. The image quality is directly related to the capabilities of the scanners to determine the spatial coordinates of the impinging gamma-rays. Accurate detector spatial 
resolution results in precise LOR assignments for each recorded coincidence event and, thus, improved reconstructed tomographic images. Nowadays, the requirements to reach high spatial resolution in PET systems are well defined and are summarized below [28][80][81][82]:

- Gamma-ray impact determination capability: For detectors based on crystal arrays this capability is directly related with the detector element width $(d)$, whereas for monolithic based detectors, it is determined by the intrinsic resolution of the detector $R_{\text {inc }}$

- Reconstruction process: as discussed in section 2.4, different reconstruction methods might result in more accurate spatial resolution.

- Parallax error: the generated gamma-rays penetrate inside the scintillators and travel some distance before they interact. Thus, if these rays penetrate the ring with an oblique angle, is more than probable that an inaccurate LOR will be generated, resulting in a blurring in the reconstruction process. This effect is further discussed in Figure 2.17.

- Electronic contribution: the readout type and accuracy may also have some impact in the position estimation and, thus, LOR decoding.

- Positron range and non-collinearity: Both are well defined physical effects in positron emission and annihilation. The term positron range refers to the distance that the positron travels upon reaching to thermal equilibrium. The term non-collinearity describes the uncertainty produced since the two emitted $511 \mathrm{keV}$ gamma-rays are not emitted strictly at a $180^{\circ}$ angle but with certain variation, due to the small residual momentum of the positron when it reaches the end of its path. For typical whole-body PET scanners, the effects of positron range and non-collinearity combined, introduce a blurring in the spatial resolution which might vary from a few tenths of a millimeter up to a few millimeters.

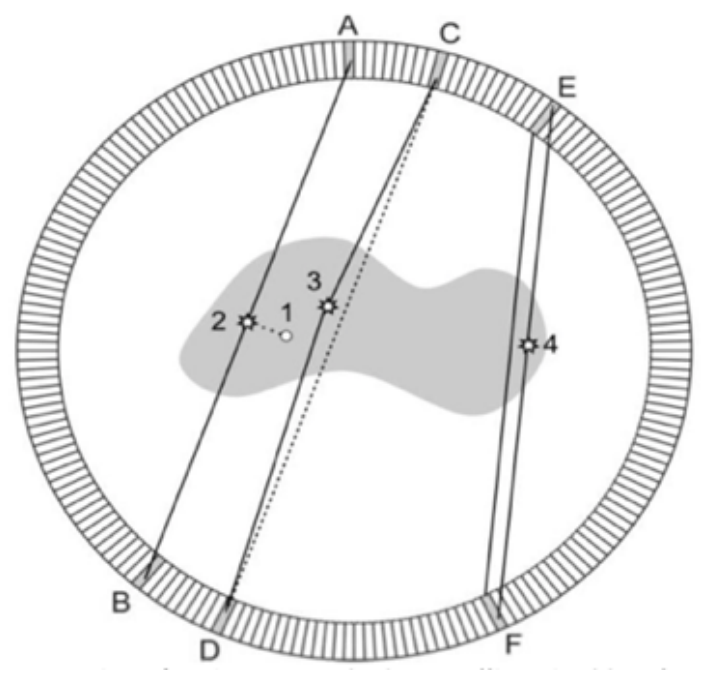

Figure 2.17. Schematic 2D representation of positron range (1-2), noncollinearity (3) and parallax (4) errors. 


\section{Role of DOI}

The influence of the parallax error due to the DOI uncertainly might show a significant effect on the spatial resolution of a system [83][84]. To give an approximation of the magnitude of the possible error, for the case of a whole-body PET scanner ( $80 \mathrm{~cm}$ of diameter), the DOI dependency leads to an approximate $40 \%$ degradation of resolution at a distance of $10 \mathrm{~cm}$ from the center of the FOV [28].

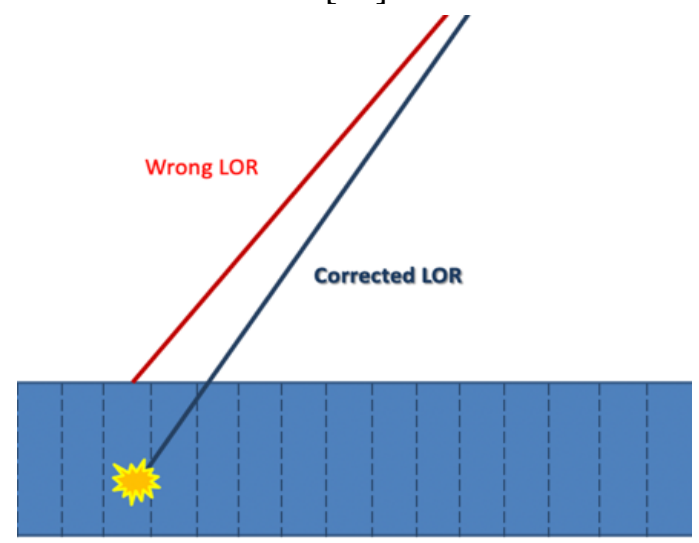

In Figure 2.18 this source of error is presented from another point of view. To overcome this, if using scintillators with lower height, this problem is directly minimized. Alternatively, one could aim to determine the depth in which the interaction was occurred (DOI). This information, if available, it can be used to generate corrected LORs, depth dependent [83].

Figure 2.18. Illustration of the parallax error effect in the LOR generation.

Decreasing the height of the crystal, although minimizes the parallax error effect, it strongly affects the number of gamma-rays stopped by each detector (a factor known as PET sensitivity). Hence, the DOI estimation, seems to be the optimal solution. As it has been demonstrated, the use of this information leads to a significant improvement in the final reconstructed image (Figure 2.19). This improvement is especially observed in small apertures system such as dedicated PET configurations or preclinical (small animal) systems in which the impact of the parallax error is more severe [28].
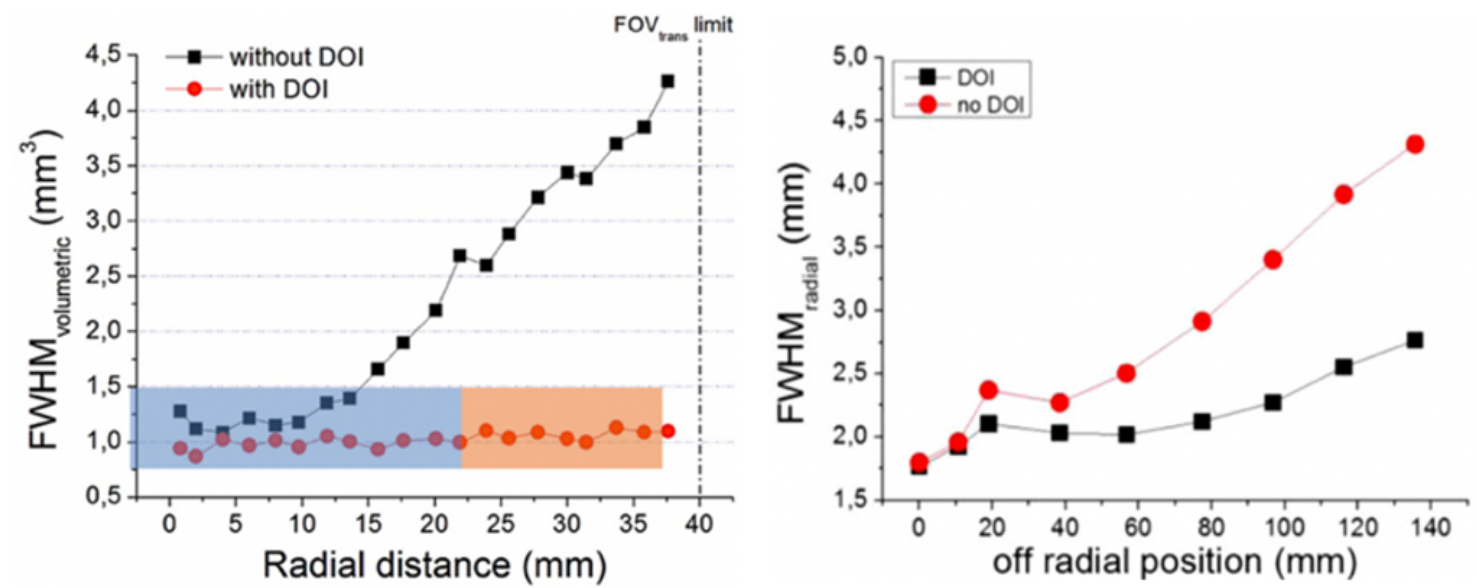

Figure 2.19. Comparison of the spatial resolution with and without the use of DOI information (as measured experimentally). Left: in the pre-clinical Albira PET (Bruker). Right: in a dedicated PET, designed for prostate imaging [85] (ProsPET, i3M). 
Generally, determining the DOI is challenging and requires specific approaches. The majority of modern clinical PET scanners are still not capable of extracting and using this information. Nevertheless, several solutions have been proposed to access to this information [86]. To give some examples, multi-layer detectors configurations [87], dual-ended readout approaches [88][89] or direct encoding with the use of monolithic crystals [90] can provide a reasonable DOI resolution [86]. However, from the development point of view, the best compromise between cost, complexity as well as spatial resolution is seen in detector configurations based on singleend readout and monolithic crystals.

\subsubsection{Timing resolution}

As already described in PET, a coincidence event is registered when two gamma-rays are simultaneously detected by two detectors inside a given coincidence time window. In most conventional systems, this window is in the range of a few nanoseconds, since conventional detectors cannot accurately detect the time arrival of the gamma-rays. This lack of time precision and the consequently necessary large coincidence window, in several cases result in the registration of false coincidence events, that affect the reconstruction accuracy [28]. In case the detectors were capable of resolving these gamma-rays time arrivals with enough precision (in the range of hundreds of picoseconds), then a shorter coincidence window is suggested, with the consequent reduction of random coincidences [91]. Besides this, when reconstruction algorithms access to a precise time information of each gamma event, they can make use of this information and better localize the event along the LOR, boosting PET performance and image quality [91][92].

Nowadays, there is a notorious interest across the community to develop detectors and methods suitable to improve the current state-of-the-art in terms of timing determination accuracy [93]. This technique, known as Time-Of-Flight (TOF), has been linked with a series of benefits for PET but also challenges [94][95]. In the next chapter we will describe the major insights of TOF-PET, discuss the state-of-the-art results and analyze the steps remained to be done.

\subsubsection{Geometries and novel configurations}

The most extended PET configuration in clinical practice is nowadays the named whole-body PET with a cylindrical geometry. Typically, this configuration only covers axially a relatively small portion of the body $(20-25 \mathrm{~cm})$. To overcome this limitation, a movable bed is integrated within the scanner, allowing the physicians to increase the axial coverage, by simply moving the patient through the scanner ring. The main drawbacks resulting from this configuration are the poor sensitivity (1-2\%), as a huge number of the generated gamma-ray pairs do not intercept the detector rings, long scan times as well as high doses to the patient [96]. 
An impressive approach has been suggested to overcome with these limitations: increasing the axial coverage of current PET systems. The first Total-Body PET scanner has been built with 2 meter axial length, increasing the detection sensitivity (see sketch in Figure 2.20 top) to up to 40 times when TOF is also provided [21][22][97]. These configurations, significantly boost system sensitivity when compared to conventional whole-body PETs. The drawback is that these types of developments are very complex and costly, however their recent introduction in the clinical practice have broaden significantly PET imaging horizons.
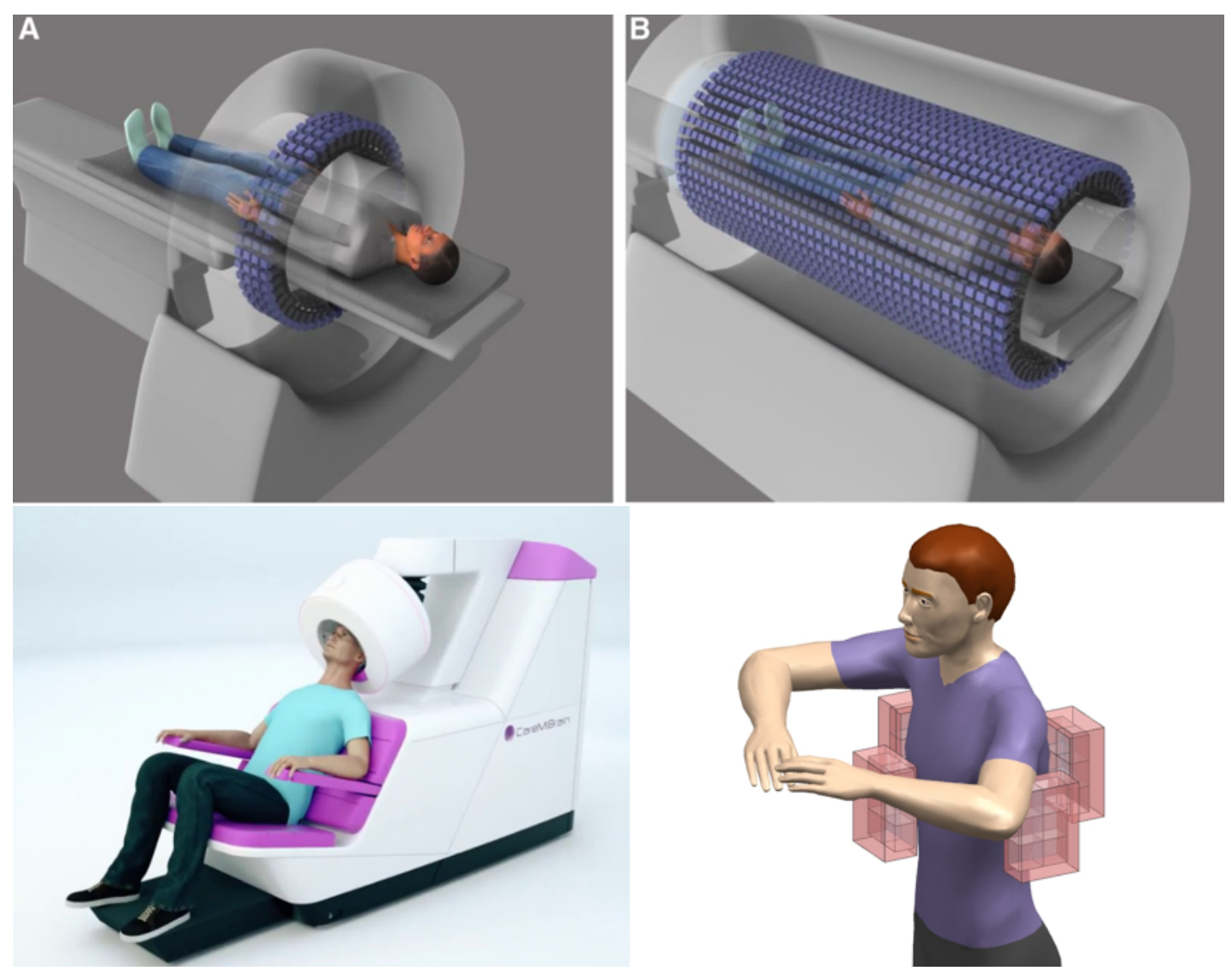

Figure 2.20. Top-Left: Conventional whole-body PET with integrated movable bed [95]. Top-Right: Total body PET [96]. Bottom Left: Dedicated PET scanner for brain imaging, commercialized by Oncovision as CareMiBrain. Bottom-Right: Dedicated PET for heart imaging with limited angle tomography.

Besides Total-Body PET imaging, several more novel PET approaches or geometries have been proposed over the years. One highly attractive approach is the organ dedicated PET imaging (see some examples in Figure 2.20 bottom) [24]. These types of configurations, permit the arrangement of the detectors closer to the object of study, resulting in inherent advantages in terms of spatial resolution, sensitivity and dose administration to the patient (and to the clinical personnel). Most of these designs follow the so-called Limited Angle Tomography (LAT) [98]. Essentially, LAT refers to PET scanners with incomplete angular coverage of the object under study (see Figure 2.20 bottom-right). This type of configuration, has the potential to enable a series of innovative diagnostic procedures towards the aim of the best possible diagnosis (e.g. 
biopsy guidance). However, the missing angular coverage, typically results in artifacts in the tomographic images [78]. This is the main reason why this technique has not yet been fully exploited. Nevertheless, as it has been demonstrated, an accurate time resolution, can significantly contribute to compensate these artifacts, and permit the development of efficient and highresolution organ-dedicated PET systems [99]. In this thesis we study detectors with accurate timing capabilities, which are also cost effective, to be used in PET systems developments with LAT geometries. 


\section{References}

[1] M. Morrocchi and A. Del Guerra, Positron Emission Tomography: alive and kicking after more than 65 years on stage. Journal of Instrumentation. 14:03 (2020), C03050

[2] H. N. Wagner, A brief history of positron emission tomography (PET). Seminars in nuclear medicine. 28 (1998), 213-220.

[3] T. Jones and D. Townsend, History and future technical innovation in positron emission tomography. Journal of Medical Imaging. 4 (2017), 011013.

[4] P. A. M Dirac, A theory of electrons and protons. Proceedings of the Royal Society of London. Series A, Containing Papers of a Mathematical and Physical Character. 126 (1930), 360-365.

[5] C. D. Anderson, The positive electron. Physical Review. 43 (1933), 491-494.

[6] R. Jean-Claude and F. Nüsslin, Marie Curie's contribution to Medical Physics. Physica Medica. 29 (2013), 423-425.

[7] S. Ruben, M. D. Kamen, W. Z. Hassid, Photosynthesis with Radioactive Carbon, Chemical Properties of the Intermediates. Journal of the American Chemical Society. 62 (1940), 3443-3450.

[8] M. N. Croll, Nuclear instrumentation. Historic perspective. Seminars in nuclear medicine, 24:1 (1994), 3-10.

[9] M. M. Ter-Pogossian, The origins of positron emission tomography. Seminars in Nuclear Medicine. 22 (1992), 140-149.

[10] F. R. Wrenn, M. L. Good, P. Handler, The use of positron-emitting radioisotopes for the localization of brain tumors. Science. 113 (1951), 525-527.

[11] G. Brownell, W. Sweet, Localization of brain tumors with positron emitters. Nucleonics. 11 (1953), 40-45.

[12] M. E. Phelps et al., Application of annihilation coincidence detection to transaxial reconstruction tomography. Journal of Nuclear Medicine. 16 (1975), 210-224.

[13] M. E. Phelps et al., Design considerations for a positron emission transaxial tomograph (Pett III). IEEE Transactions on Nuclear Science. 23 (1976), 516-522.

[14] C. W. Williams, M. C. Crabtree, S. G. Burgiss, Design and performance characteristics of a positron emission computed axial tomograph-ecat®-II. IEEE Transactions on Nuclear Science. 26 (1979), 619-627.

[15] R. M. E Sabbatini, The PET Scan: A New Window Into the Brain, Brain \& Mind Magazine, 2007.

[16] Jun Zhang and Michael V. Knopp, Advances in PET. Springer, Cham (2020). 
[17] I. Rausch et al., Performance evaluation of the Vereos PET/CT system according to the NEMA NU2-2012 standard. Journal of Nuclear Medicine. 60 (2019), 561-567.

[18] J. Van Sluis et al., Performance characteristics of the digital biograph vision PET/CT system. Journal of Nuclear Medicine. 60 (2019), 1031-1036.

[19] G Delso et al., Performance measurements of the Siemens mMR integrated whole-body PET/MR scanner. Journal of Nuclear Medicine. 52:12 (2011), 1914-1922.

[20] J. M. Benlloch, et al., The MINDVIEW project: First results, European Psychiatry. 50 (2018), 21-27.

[21] R. D. Badawi et al., EXPLORER, an ultrasensitive total-body PET scanner: Application feasibility simulations. Molecular Imaging and Biology. 15 (2013), S282-S283.

[22] X. Zhang et al., Total-body dynamic reconstruction and parametric imaging on the uexplorer. Journal of Nuclear Medicine. 61 (2020), 285-291.

[23] E. Garutti, EndoTOFPET-US a novel multimodal tool for endoscopy and Positron Emission Tomography. IEEE Nuclear Science Symposium and Medical Imaging Conference Record (NSS/MIC), Anaheim, 2012, 2096-2101

[24] A. J. Gonzalez, F. Sanchez, J. M. Benlloch, Organ-Dedicated Molecular Imaging Systems. IEEE Transactions on Radiation and Plasma Medical Sciences, 2 (2018), 388403.

[25] K. Serdons, A. Verbruggen, G. M. Bormans, Developing new molecular imaging probes for PET. Methods. 48 (2009), 104-111.

[26] A. Shukla and U. Kumar, Positron emission tomography: An overview. Journal of Medical Physics. 31 (2006), 13-21.

[27] G. F. Knoll, Radiation Detection and Measurement. John Wiley \& Sons, New York, 3rd edition (2000).

[28] S. Cherry, J. Sorenson, M. Phelps, Physics in Nuclear Medicine. Elsevier Inc, 7 (2012) 87-106.

[29] G. Madhavan, Ionizing Radiation Detectors for Medical Imaging, IEEE Engineering in Medicine and Biology Magazine. 25 (2006), 10-10.

[30] D. D. Nolting, M. L. Nickels, N. Guo, W. Pham, Molecular imaging probe development: a chemistry perspective. American journal of nuclear medicine and molecular imaging. 2 (2012), 273-306.

[31] D. J. Schlyer, PET tracers and radiochemistry. Annals of the Academy of Medicine Singapore. 33 (2004), 146-154.

[32] M. E. Phelps, S. R. Cherry, M. Dahlbom, PET: Physics, instrumentation, and scanners Springer New York (2006). 
[33] G. Bizarri, Scintillation mechanisms of inorganic materials: From crystal characteristics to scintillation properties. Journal of Crystal Growth. 312 (2010), 1213-1215.

[34] W. R. Leo, D. G. Haase, Techniques for Nuclear and Particle Physics Experiments. American Journal of Physics. 58 (1990), 1216-1217.

[35] C. L. Melcher, Scintillation crystals for PET. Journal of Nuclear Medicine. 41 (2000), 1051-1055.

[36] P. Lecoq et al., Inorganic Scintillators for Detector Systems. Springer-Verlag, 2006.

[37] C. W. E. Van Eijk, Inorganic scintillators in medical imaging. Physics in Medicine and Biology. 47 (2002), 8.

[38] G. Borghi, V. Tabacchini, D. R. Schaart, Towards monolithic scintillator based TOF-PET systems: Practical methods for detector calibration and operation. Physics in Medicine and Biology. 61 (2016), 4904-4928.

[39] S. E. Derenzo et al., The quest for the ideal inorganic scintillator. Nucl. Instrum. Methods Phys. Res. A. 505 (2003), 111-7.

[40] P. Lecoq, Development of new scintillators for medical applications. Nucl. Instrum. Methods Phys. Res. A. 809 (2016), 130-139.

[41] Hamamatsu Photonics Product Catalog: "Photomultiplier Tube Modules" (2005).

[42] S. Flyckt and C. Marmonier, Photomultiplier tubes: Principles and applications. Phonis, Brive, France, 2002.

[43] F. Acerbi and S. Gundacker, Understanding and simulating SiPMs. Nucl. Instrum. Methods Phys. Res. A. 487 (2002), 926 (2019), 16-35.

[44] D. Renker and E. Lorenz, Advances in solid state photon detectors. Journal of Instrumentation. 4 (2009), P04004

[45] D. Renker, New trends on photodetectors. Nucl. Instrum. Methods Phys. Res. A. 571 (2007), 1-6.

[46] C. L. Kim, G. C. Wang, S. Dolinsky, Multi-pixel photon counters for TOF PET detector and its challenges. IEEE Transactions on Nuclear Science. 56 (2009), 2580-2585.

[47] D. Renker, Geiger-mode avalanche photodiodes, history, properties and problems. Nucl. Instrum. Methods Phys. Res. A. 567 (2006), 48-56.

[48] S. Gundacker and A. Heering, The silicon photomultiplier: fundamentals and applications of a modern solid-state photon detector. Physics in Medicine and Biology. 65 (2020), 17TR01.

[49] E. Auffray et al., Characterization studies of silicon photomultipliers and crystals matrices for a novel time of flight PET detector. Journal of Instrumentation. 10 (2015), P06009. 
[50] www.ketek.net

[51] T. Frach et al., The digital silicon photomultiplier system architecture and performance evaluation. IEEE Nuclear Science Symposium Conference Record (2010), 1722-1727.

[52] T. Frach et al., The digital silicon photomultiplier - Principle of operation and intrinsic detector performance. IEEE Nuclear Science Symposium Conference Record (2009), 1959-1965.

[53] J. L. Humm, A. Rosenfeld, A. Del Guerra, From PET detectors to PET scanners. European Journal of Nuclear Medicine and Molecular Imaging. 30 (2003), 1574-1597.

[54] K. Shimazoe et al., Dynamic time over threshold method. IEEE Transactions on Nuclear Science. 59 (2012), 3213-3217.

[55] Y. Tian, H. Takahashi, K. Shimazoe, Characteristics of an energy-resolving system using dynamic time over threshold method. IEEE Transactions on Nuclear Science. 62 (2015), 1798-1804.

[56] W. S. Choong et al., A front-end readout Detector Board for the OpenPET electronics system. Journal of Instrumentation. 10 (2015), T08002.

[57] H. O. Anger, Scintillation Camera. Review of Scientific Instruments, 29 (1958), 27-33.

[58] S. Siegel, R. W. Silverman, Y. Shao, S. R. Cherry, Simple charge division readouts for imaging scintillator arrays using a multi-channel PMT. IEEE Transactions on Nuclear Science. 43 (1996), 1634-1641.

[59] A. J. Gonzalez et al., Simulation study of resistor networks applied to an array of 256 SiPMs. IEEE Transactions on Nuclear Science. 60 (2013), 592-598.

[60] V. Popov et al., Readout Electronics for Multianode Photomultiplier Tubes With Pad Matrix Anode Layout. IEEE Nuclear Science Symposium Conference Record, Portland, 3 (2003), 2156-2159.

[61] V. Popov et al., Analog readout system with charge division type output. IEEE Nuclear Science Symposium Conference Record, San Diego, 4 (2001), 1937-1940.

[62] M. M. Vai, W. S. Song, B. M. Tyrrell, High Performance Embedded Computing Handbook: A Systems Perspective. CRC Press (2008), 191-215.

[63] A. Di Francesco et al., TOFPET2: A high-performance ASIC for time and amplitude measurements of SiPM signals in time-of-flight applications. Journal of Instrumentation. 11:03 (2016), C03042.

[64] TOFPET2 ASIC Evaluation kit - Hardware User Guide (v1.2), v1.2, PETsys Electronics SA., 2018.

[65] I. Sarasola et al., A comparative study of the time performance between NINO and FlexToT ASICs. Journal of Instrumentation. 12 (2017), P04016. 
[66] A. Aguilar et al., Preliminary characterization of ASIC-based detectors for TOF-PET applications. IEEE Nuclear Science Symposium Conference Record, Strasbourg (2016) $1-5$.

[67] J. M. Monzo et al., PETIROC2 based readout electronics optimization for Gamma Cameras and PET detectors. Journal of Instrumentation. 12 (2017), 1-8.

[68] S. Ahmad et al., Triroc: A Multi-Channel SiPM Read-Out ASIC for PET/PET-ToF Application. IEEE Transactions on Nuclear Science. 62 (2015), 664-668.

[69] J. F. Genat et al., Signal processing for picosecond resolution timing measurements. Nucl. Instrum. Methods A. 607 (2009), 387-393.

[70] S. Tong, A. M. Alessio, P. E. Kinahan, Image reconstruction for PET/CT scanners: Past achievements and future challenges. Imaging in Medicine. 2 (2010), 529-545.

[71] A. J. Reader, H. Zaidi, Advances in PET Image Reconstruction. PET Clinics. 2 (2007), 173-190.

[72] E. Busemann Sokole et al., Routine quality control recommendations for nuclear medicine instrumentation. European Journal of Nuclear Medicine and Molecular Imaging. 37 (2010), 662-671.

[73] L. A. Shepp and Y. Vardi, Maximum Likelihood Reconstruction for Emission Tomography. IEEE Transactions on Medical Imaging. 1 (1982), 113-122.

[74] H. M. Hudson and R. S. Larkin, Accelerated Image Reconstruction Using Ordered Subsets of Projection Data. IEEE Transactions on Medical Imaging. 13 (1994), 601-609.

[75] T. E. Peterson and H. C. Manning, Molecular imaging: 18F-FDG PET and a whole lot more. Journal of Nuclear Medicine Technology, 37:3 (2009), 151-161.

[76] A. Alavi and M. Reivich, Guest editorial: The conception of FDG-PET imaging. Seminars in Nuclear Medicine. 32 (2002), 2-5.

[77] R. D. Badawi, IEEE 2020 EXPLORER Total Body PET: Progress, Challenges and Opportunities, IEEE Nuclear Science Symposium, Manchester, 2020.

[78] S. Vandenberghe et al., Recent developments in time-of-flight PET. EJNMMI Physics. 3:3 (2016), 3 .

[79] R. D. Badawi et al., First human imaging studies with the explorer total-body PET scanner. Journal of Nuclear Medicine. 60 (2019), 299-303.

[80] W. W. Moses, Fundamental limits of spatial resolution in PET. Nucl. Instrum. Methods A. 648 (2011), S236-S240.

[81] C. S. Levin and E. J. Hoffman, Calculation of positron range and its effect on the fundamental limit of positron emission tomography system spatial resolution. Physics in Medicine and Biology. 44 (1999), 781-799. 
[82] K. Shibuya et al., Limit of Spatial Resolution in FDG-PET due to Annihilation Photon Non-Collinearity. IFMBE Proceedings, 14 (2007), 1667-1671.

[83] T. Yamaya, PET Imaging Innovation by DOI Detectors, Perspectives on Nuclear Medicine for Molecular Diagnosis and Integrated Therapy. Springer (2016), 39-49.

[84] M. S. Brown et al., Influence of depth of interaction upon the performance of scintillator detectors. PLOS ONE. 9 (2014), e98177.

[85] G. Cañizares et al., Pilot performance of a dedicated prostate PET suitable for diagnosis and biobsy guidance. EJNMMI Physics. 7:31(2020), 38.

[86] I. Mohammadi, et. al., Minimization of parallax error in positron emission tomography using depth of interaction capable detectors: Methods and apparatus. Biomedical Physics and Engineering Express. 5 (2019).

[87] T. Tsuda et al., A four-layer depth of interaction detector block for small animal PET. IEEE Transactions on Nuclear Science. 51 (2004), 2537-2542.

[88] M. Morrocchi et al., Depth of interaction determination in monolithic scintillator with double side SiPM readout. EJNMMI Physics. 4:1 (2017), 180.

[89] S. J. Lee et al., Preliminary experimental results of a quasi-monolithic detector with DOI capability for a small animal PET. Nucl. Instrum. Methods Phys. Res. A. 621 (2010), 590-594.

[90] A. González-Montoro et al., Novel method to measure the intrinsic spatial resolution in PET detectors based on monolithic crystals. Nucl. Instrum. Methods Phys. Res. A. 920, 58-67 (2019).

[91] M. Conti, Focus on time-of-flight PET: The benefits of improved time resolution. European Journal of Nuclear Medicine and Molecular Imaging. 38 (2011), 1147-1157.

[92] M. Conti, State of the art and challenges of time-of-flight PET. Physica Medica. 25 (2009), 1-11.

[93] M. Conti, B. Bendriem, The new opportunities for high time resolution clinical TOF PET. Clinical and Translational Imaging. 7 (2019), 139-147.

[94] P. Lecoq, Pushing the Limits in Time-of-Flight PET Imaging. IEEE Transactions on Radiation and Plasma Medical Sciences. 1 (2017), 473-485.

[95] S. Surti, Update on time-of-flight PET imaging. Journal of Nuclear Medicine. 56 (2015), 98-105.

[96] S. R. Cherry et al., Total-body PET: Maximizing sensitivity to create new opportunities for clinical research and patient care. Journal of Nuclear Medicine. 59 (2018), 3-12.

[97] S. Vandenberghe, P. Moskal, J. S. Karp, State of the art in total body PET. EJNMMI Physics. 35 (2020), 7. 
[98] S. Surti, J. S. Karp, Design considerations for a limited angle, dedicated breast, TOF PET scanner. Physics in Medicine and Biology. 53 (2008), 2911-2921.

[99] P. Gravel, Y. Li, S. Matej, Effects of TOF Resolution Models on Edge Artifacts in PET Reconstruction from Limited-Angle Data. IEEE Transactions on Radiation and Plasma Medical Sciences, 1-1 (2020). 


\section{Chapter 3}

\section{Time-of-Flight in PET}

Before proceeding with the main part of this thesis, it is necessary to discuss and analyze all factors related with TOF information from the detector development point of view. As in every engineer project, also in TOF-PET detectors, requirements and limitations have to be considered and trade-offs to be made. In this chapter we aim to shed light on all factors related with the timing performance, as well as to expand aforementioned descriptions. Experimental results, some not yet published, will be presented aiming to support the statements and facilitate understanding.

\subsection{Time-of-Flight technique}

Since the first PET developments, the value of an accurate detection of the time arrival of the $511 \mathrm{keV}$ gamma-rays has been well-defined. Some interesting early perspectives can be found in the works [1]-[5]. This recognition enabled and motivated a parallel effort to improve the timing detection accuracy, and extrapolate the TOF information [6][7]. The access to such TOF information takes PET imaging and instrumentation a step further. Essentially, TOF describes the accurate determination of the time arrival of the detected gamma-rays and its usage to better estimate the point along the LOR where the positron-electron annihilation occurred [6]. The timing precision is related to the space uncertainty by:

$$
\Delta x=\frac{c \cdot \Delta t}{2} \text {, with } c \text { the speed of light and } \Delta t \text { the timing resolution. }
$$

An accurate determination of this information is still challenging and requires specific approaches or trade-offs [8][9]. Over the last decade there have been significant improvements in photodetection, electronics and scintillation mechanisms, making it possible to enhance these capabilities [10]. Nowadays, even the most advanced detectors are only capable to provide the time arrival information with a precision of hundreds of picoseconds, which is directly related with detectors precision capabilities to determine the value $\Delta t$, or as it known, their Coincidence Timing Resolution (CTR). This results in an uncertainty $\Delta x$ (in the range of several centimeters) [7][9][10] (see Table 3.1). While the CTR performance, and the factors to which it depends will be discussed in the next subsections, in the next paragraphs, we discuss the benefits related with an accurate TOF information. 


\subsection{Benefits related with TOF}

If detectors were capable of resolving the photons time arrival with a precision of 10 picoseconds, then the annihilation event could be assessed along the LOR with an uncertainty of only 1.5 millimeter, making even unnecessary complex reconstruction algorithms [8][11][12].

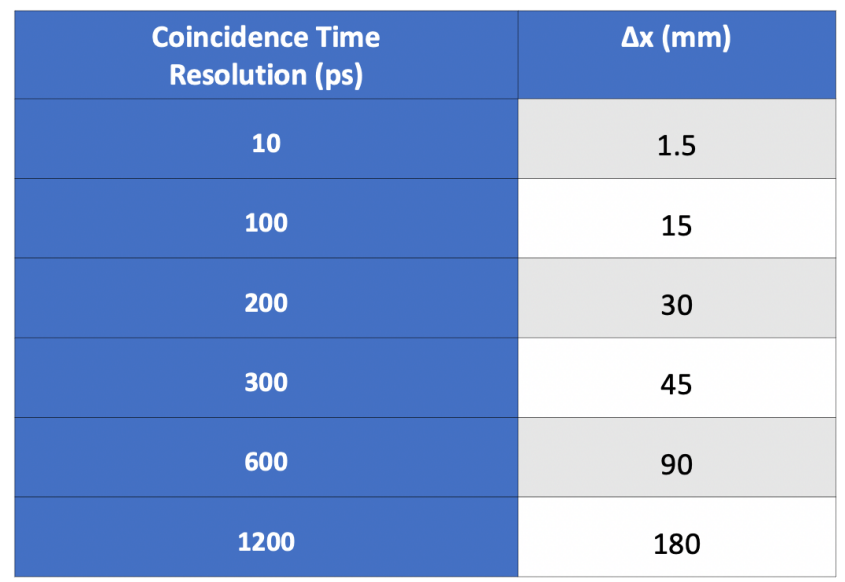

Table 3.1. Spatial uncertainty in the localization of the annihilation point along the LOR as a function of the CTR.

Although, this time resolution is not feasible yet and current detector time capabilities are limited to values at least one order of magnitude above, still TOF is extremely useful in PET practice. When TOF is enabled, a probability function along the LOR is used during the reconstruction process (see Figure 3.1 and Table 3.1) [10][13]. This function follows a Gaussian distribution centered on the position provided by the time differences of the detected gamma-rays with a width corresponding to the system CTR. Thus, only a segment of the LOR constrains the probable point of interaction, in contrast to the case without CTR information in which all points (voxels) along the LOR are equally probable to allocate the annihilation event [14].

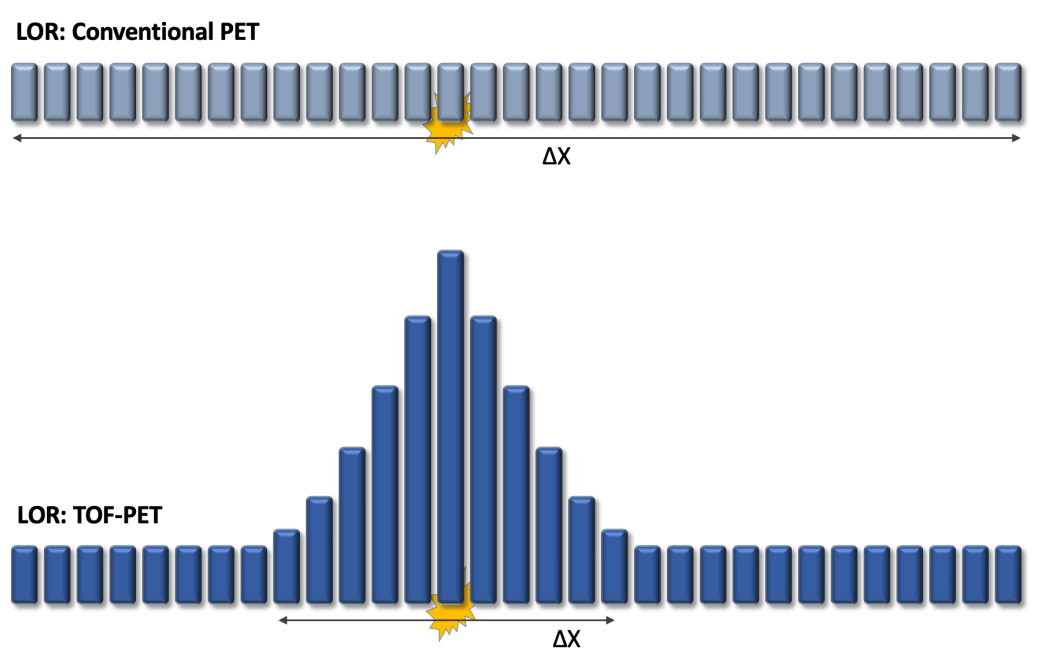

Figure 3.1. Annihilation event localization inside the LOR in the case of conventional and TOF-PET. 


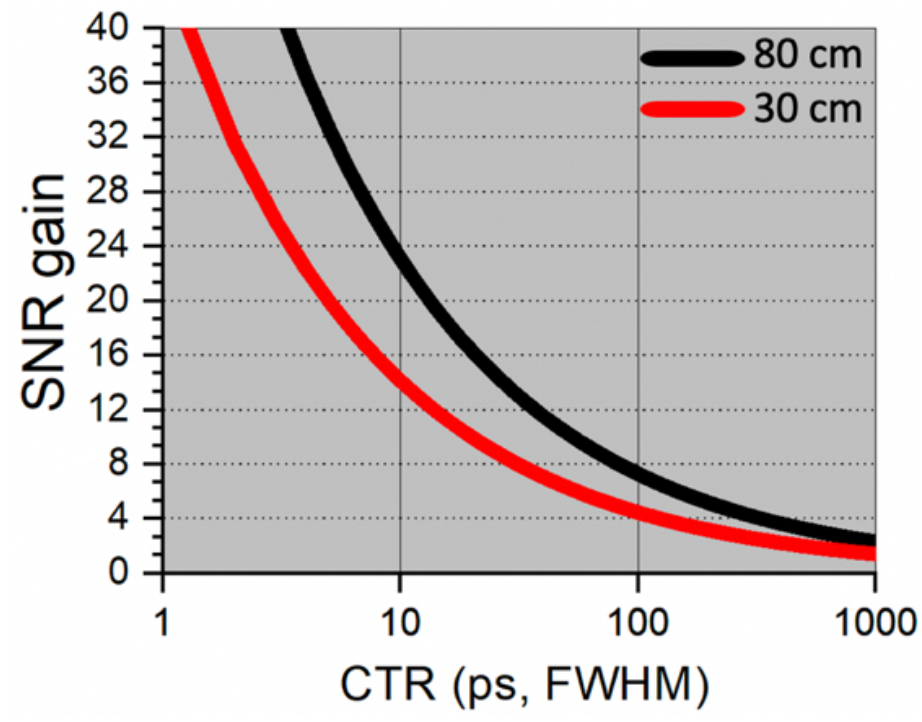

Figure 3.2. Improvement in the Signal-To-Noise ratio compared to non-TOF as a function of the time resolution, for two different scanner diameters (D).
The main benefit resulting from accurate TOF information is the direct improvement in the image SNR. TOF information makes it possible to generate images less influenced by statistical noise and with higher contrast to noise ratio [6][12]. The improvement in SNR when TOF is available is described using the following formula:

$$
\frac{\text { SNR TOF }}{\text { SNR nonTOF }}=\sqrt{\frac{2 D}{c \cdot C T R}}
$$

where $D$ is the diameter of the

object to be imaged. In Figure 3.2, the expected SNR improvement as a function of the CTR is presented [6][12].

The improvement in the SNR is translated to the clinical practice in the form of the aforementioned noise reduction. Herein, TOF enables a virtual increase in the effective sensitivity permitting: i) reduction of scanning time and/or dose, ii) better image quality for a given dose and scanning time iii) dosimetry with extremely low statistics and iv) the development of new PET concepts such as scanners with limited angle coverage, to name but a few [7][10][12].

Aiming to support the last statements and to demonstrate in practice that TOF can indeed permit the development of a PET configuration with partial coverage of the object of study, we present some unpublished results obtained with a TOF-PET prototype developed by the candidate. The system is composed by two plane panels faced one to each other, following a LAT geometry, while is capable of providing a CTR as good as 238 ps FWHM for all 3072 integrated readout channels [15]. As it can be appreciated in Figure 3.3, when TOF information is considered during the reconstruction process, noise and artifacts are faded from the generated images and the overall image quality is improved [15]. Although further improvement is expected following the system optimization, already from these early findings, the benefits of TOF in these types of configurations are profound. 

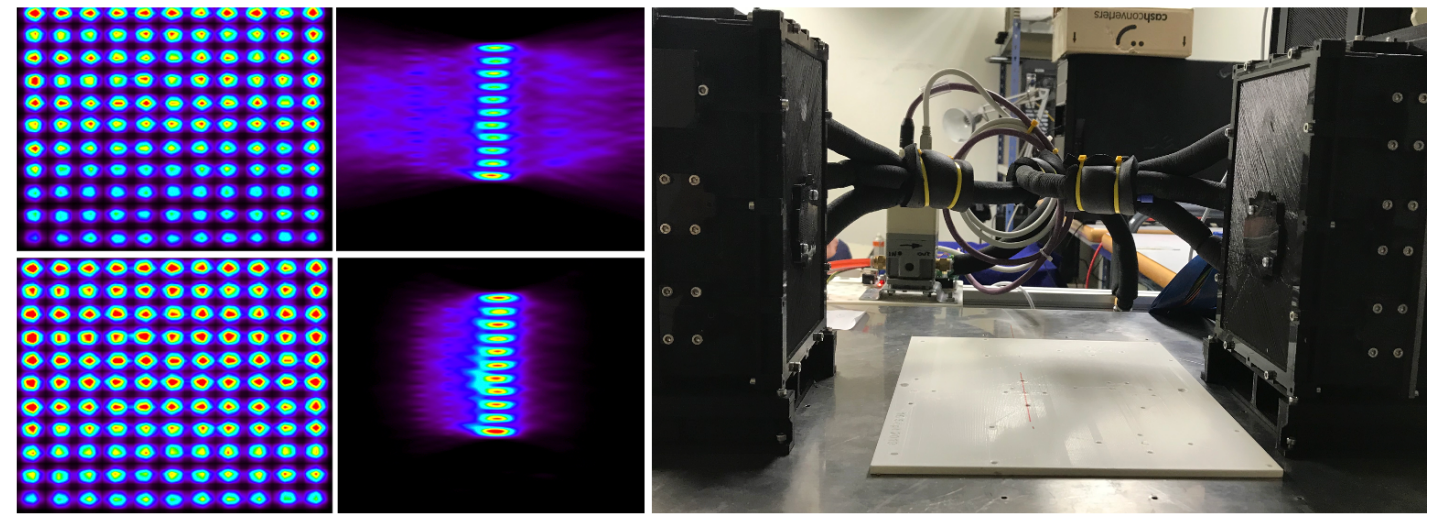

Figure 3.3. Left: Images of a source array $11 \times 11\left(1 \times 1 \mathrm{~mm}^{2}\right.$ each source size $)$. Top. obtained without the use of the timing information showing the sagittal and transverse views. Bottom. using TOF information for the same views. Images were obtained using the TOF-PET prototype named CardioPET (i3M). Right: CardioPET evaluation set-up.

\subsection{Brief evolution of TOF-PET imaging}

Although the benefits correlated with a good timing resolution were well known since PET first developments, it was not until mid-2000s when the first TOF-PET scanners were introduced to the market [10] (Figure 3.4). The very first, was commercialized by Philips back in 2006 under the name Gemini TF PET/CT, and is capable of reaching a time resolution of 585 picoseconds [16]. Shortly after but in the same period, two more scanners were presented by General Electric (named Discovery 690) [17] and by Siemens (mCT) [18], which both could reach slightly better timing resolution in the range of 520-550 picoseconds. This series of almost concurrent developments should not be considered aleatory but is directly related with the arrival of the novel back-in-the-time scintillators, lutetium-based, which were fast enough to facilitate the extrapolation of accurate timing information [10]. However, no significant improvement was seen in terms of timing resolution up to the era, in which the SiPMs substituted the PMTs [19]-[22].

With the arrival of SiPM, the barriers in timing resolution seemed to brake. In 2014, a time resolution as good as 345 picoseconds was presented in a Philips prototype scanner [23]. Shortly after that, the very same company commercialized the so-called scanner Vereos PET with a TOF performance of 310 picoseconds using digital SiPM technology [24]. General Electric also presented a PET, capable of reaching the 375 picoseconds [25]. Just recently, in 2018, Siemens announced their latest development in PET imaging, the scanner named Vision, which exhibits a timing resolution of 214 picoseconds FWHM, consisting up to today the state-of-the-art in terms of timing capabilities in commercially available systems [26][27].

Nevertheless, concurrently to these developments, in the research field several steps have been made proving an achievable roadmap towards even a better timing performance. In the literature one can find several works, including the ones that are presented in this thesis, where timing resolutions even below 100 picoseconds FWHM are achieved at the detector level 
[12][28][29][30][34]. Although these achievements are an important guide and motivation for researchers and engineers, a series of limitations have not currently allowed their translation into efficient detector solutions and following that into commercially available systems.

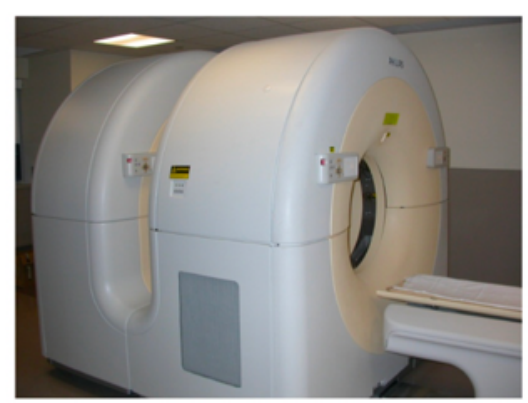

Phillips Gemini TF

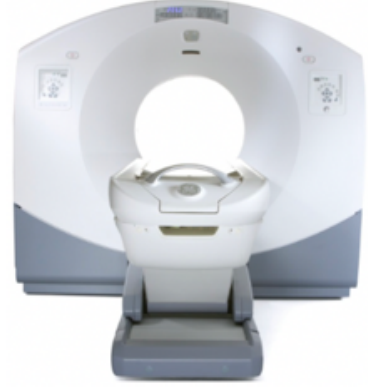

GE Discovery 690

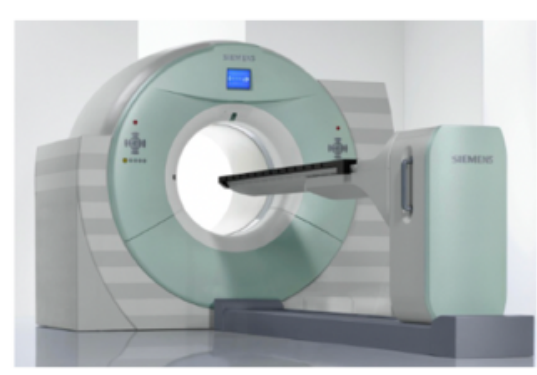

Siemens mCT

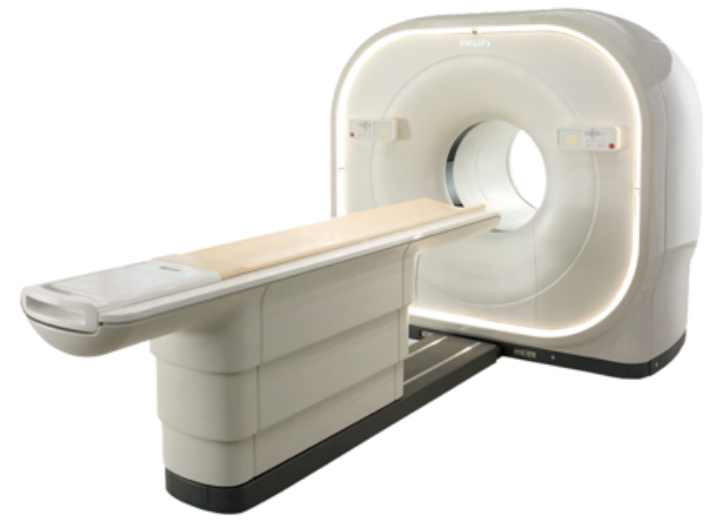

Phillips Vereos

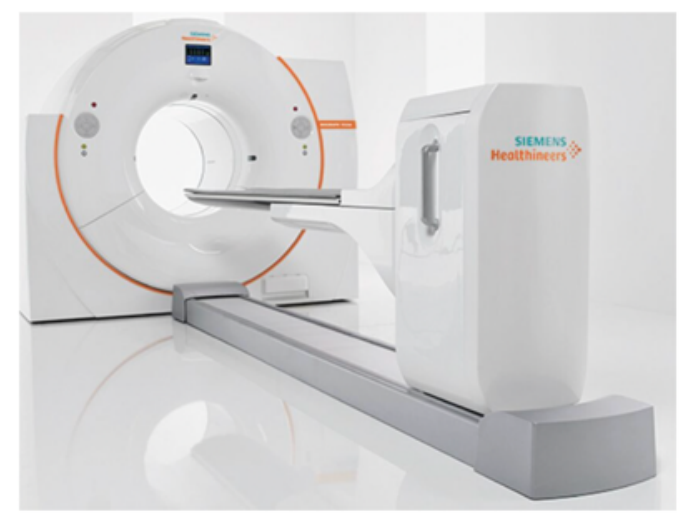

Siemens Vision

Figure 3.4. Commercial TOF-PET scanners developed and exploited in the clinical practice over the years.

Nevertheless, concurrently to these developments, in the research field several steps have been made proving an achievable roadmap towards even a better timing performance. In the literature one can find several works, including the ones that are presented in this thesis, where timing resolutions even below 100 picoseconds FWHM are achieved at the detector level [12][28][29][30][34]. Although these achievements are an important guide and motivation for researchers and engineers, a series of limitations have not currently allowed their translation into efficient detector solutions and following that into commercially available systems.

\subsection{TOF-PET: requirements and limitations}

All independent components of a PET detector contribute to its performance and to such of the whole system (Figure 3.5) [11][13]. In the following subsections, we will describe all correlated factors and summarize all knowledge related with timing applications in state-of-theart PET. 

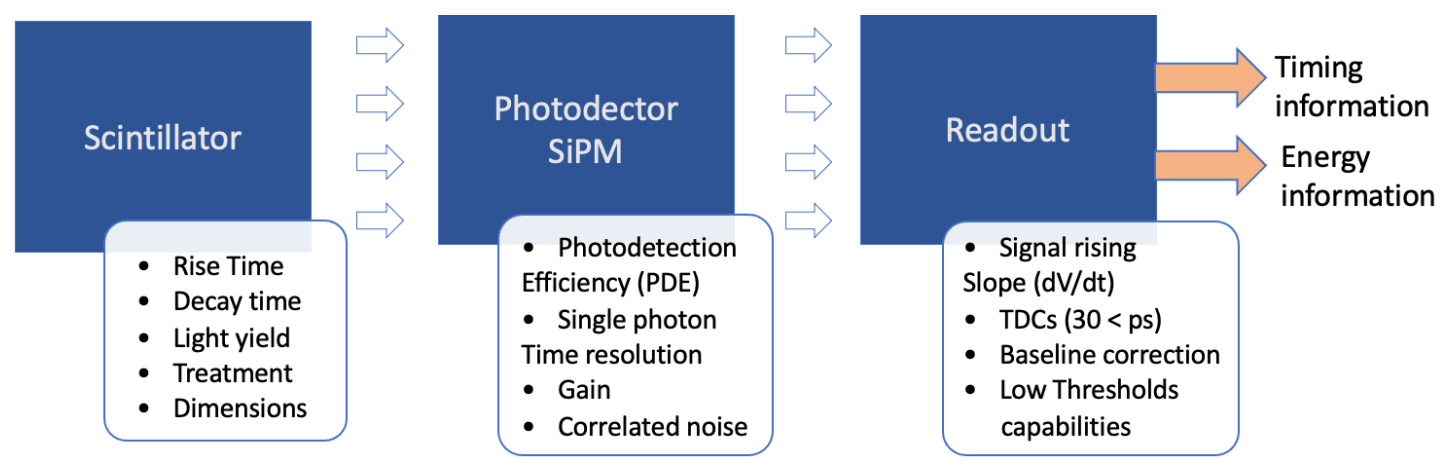

Figure 3.5. Schematic representation of TOF-PET detectors and their main requirements.

\subsubsection{Scintillation material}

In a TOF-PET detector, the scintillator type itself infers intrinsic characteristics related with the timing capabilities [12]. As it was already discussed in chapter 2 (section 2.3.1), diverse types of scintillators exist but just a few of them are suitable for TOF-PET applications. In particular, a TOF-PET detector requires a scintillator with a short rise time compared to the decay time and with a high light yield. Additionally, the higher the initial scintillation light intensity it is, the more probable gets a good time resolution [30]-[32]. Those critical characteristics will permit the generation of the highest number of optical photons possible in a very short time and consequently, to the generation of a pulse with a sharp rising edge, facilitating the timestamp determination.

Aside from the scintillator type, its geometry also plays a key role in the detector timing capabilities. The crystal geometry is directly related with the light transport from the gamma-ray interaction point to the photosensor. Due to the isotropic emission of the generated optical photons, they might follow different paths before reaching the photosensor. Herein, internal reflections with the scintillator faces occur during this transit, affecting the required initial photon intensity. This is known as Optical-Transit-Time-Spread (OTTS) and might strongly influence the timing determination [33]. Moreover, the DOI of the gamma-ray also shows a significant influence in the timing resolution, as optical photons reach the photosensors at different time periods [33]. Moreover, thick crystals increase the probability for photons to be absorbed through a random deletion process, impacting the light transfer efficiency (LTE) to the photosensors [12][33]. Therefore, the larger the scintillator crystal dimensions, the more probable becomes a poorer time resolution. In Figure 3.6, both the DOI dependency and OTTS are illustrated while in Figure 3.7, we experimentally demonstrate using an ASIC readout and SiPM photosensors how the detector timing capabilities are affected by increasing the scintillator crystal length.

Lastly, it is worth mentioning that in order to enhance the total scintillation light output and, therefore, improve the timing performance, a scintillator surface treatment with high reflectivity 
materials is needed [34]. Teflon, white paint, Enhanced Specular Reflectors (ESR) and BaSO4 layers are the most used in TOF-PET detector configurations.

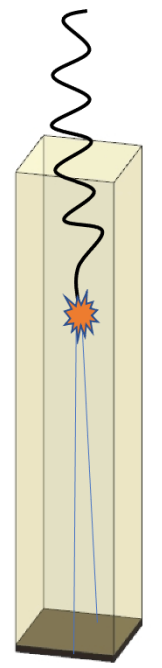

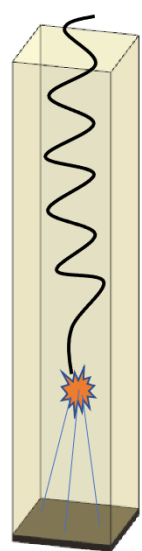

DOI dependency
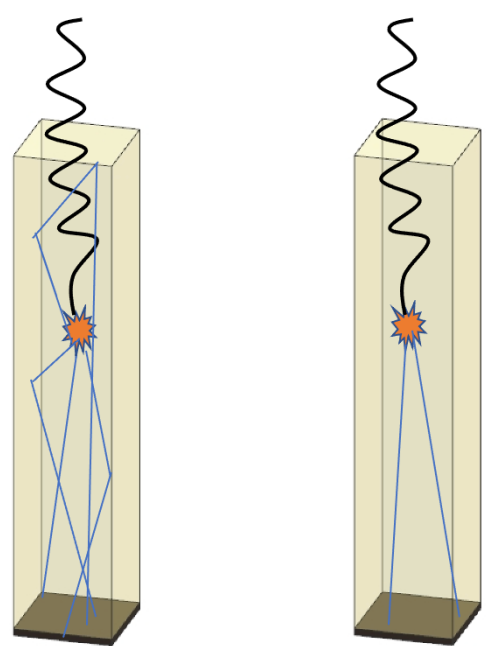

OTTS dependency

Figure 3.6. Schematic representation of DOI and OTTS dependency with optical photons path.

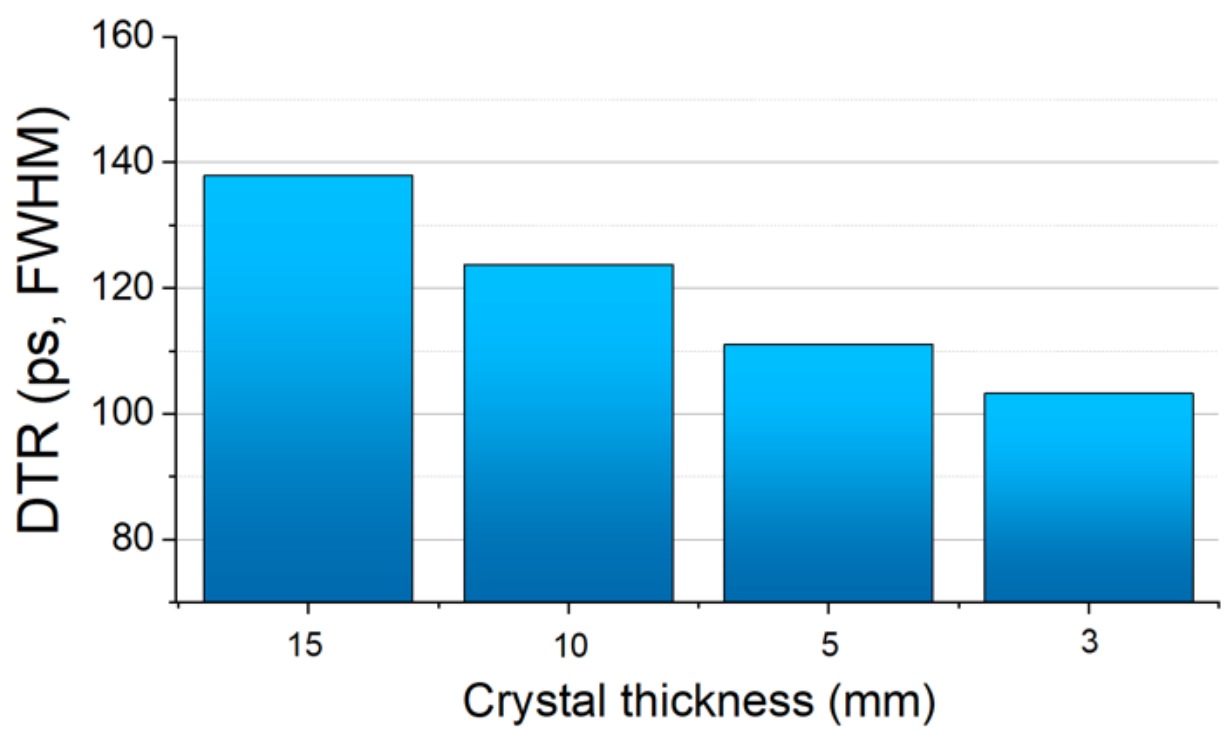

Figure 3.7. Detector Time Resolution (DTR) as a function of the crystal thickness for a $3 \times 3 \mathrm{~mm}^{2}$ size pixel of Cadoped LYSO, measured with the TOFPET2 ASIC and a pair of S13360 Hamamatsu SiPM arrays.

\subsubsection{Photo-detection}

Despite the big advances in SiPM technology during the last decade, photo-detection still shows one big limitation; from the whole number of optical photons exiting the scintillator towards the SiPMs, only a fraction nearing 50\% are eventually detected [6][12]. This effect is 
called photo-detection efficiency (PDE) and it is one of the most significant factors influencing the timing capabilities [35]. Considering that every single photon is a possible carrier of timing information, the need for a photodetector with the maximum sensitivity is profound. Herein, the fill factor, resulting from the SiPM active area and the cell size, should also be chosen accordingly [36][37]. Other SiPM characteristics such as, gain, capacitance or crosstalk, among others, might sometimes also affect the timing results. Summarizing, there are different types of SiPMs and, therefore, they can impact the timing performance so, a proper selection is important.

In the literature, we can find a broad description of experimental and simulated studies in which SiPMs are evaluated. In Table 3.2 we list the results obtained in a study where the authors have compared most of common SiPMs suitable for TOF applications in PET, both in terms of SPTR and CTR, using a high-frequency readout [38]. However, these studies are only indicatives, since as already introduced, depending on the scintillator type, size or coupling method, as well as SiPMs characteristics, different results might be reached. Thus, the evaluation of several SiPMs might be necessary in order to find the most suitable one.

\begin{tabular}{|c|c|c|c|}
\hline SIPM type & SPTR (FWHM, ps) & Weighted PDE (\%) & CTR (FWHM, ps) \\
\hline HPK S13360 & $144 \pm 7$ & 59 & $75 \pm 3$ \\
\hline HPK S14160 & $126 \pm 5$ & 56.5 & $74 \pm 3$ \\
\hline KETEK PM3325 & $184 \pm 8$ & 53 & $86 \pm 3$ \\
\hline KETEK PA3350 & $92 \pm 5$ & 51 & $70 \pm 3$ \\
\hline SensI FJ30035 & $132 \pm 6$ & 50 & $76 \pm 3$ \\
\hline Broadcom & $112 \pm 5$ & 55 & $69 \pm 3$ \\
\hline FBK NUV-HD & $92 \pm 5$ & 59 & $63 \pm 3$ \\
\hline FBK NUV-HD no resin & $85 \pm 5$ & 59 & $58 \pm 3$ \\
\hline
\end{tabular}

Table 3.2. SiPM timing performance overview of the state-of-the-art SiPMs, as measured with a high-performance readout. The presented CTR values are the best ones found, with $2 \times 2 \times 3 \mathrm{~mm}^{3}$ LSO: Ce: $0.4 \% \mathrm{Ca}$ crystals, at the SiPM bias voltage for which the SPTR and PDE values are given. Extracted and modified from [38].

\subsubsection{Readout electronics}

Once the SiPM signals are fed to the readout circuitries, typically following some first processing (such as pulse shaping and/or pre-amplification), are processed in order to provide energy and timing information. As it was mentioned before, the timing determination, thus the generation of the timestamp in which the first scintillation photons arrived to the photosensors, is 
a complex task [39][40]. Several forms of noise or jitter, intrinsic to this procedure, typically result in a loss of precision in the determination of the time of occurrence of the incoming pulse [41]. The main source of errors found during the signal time decoding are [42]:

- Jitter, the time uncertainty provoked by noise and statistical fluctuations of the gamma signals

- Time-walk, which can be defined as the timing error produced by the variation of the amplitude and shape of the input pulses, during the timestamp generation

- Drift, the timing error produced by component aging and temperature variations

- Input signal non-linearity

Although the last two sources of error are negligible compared to the jitter and time-walk, all of them influence and might result in a deterioration of the time capabilities of a TOF-PET detector. Fortunately, as we will later discuss, the time-walk can also be compensated (partially) by applying calibration methods or by selecting a suitable time pick-off circuit method. Up to now, the time decoding in PET has mainly been carried out by two types of pick-off circuits. These are the Leading-Edge Discriminator (LED) and the Constant Fraction Discriminator (CFD), which both are briefly described in the following [41][42].
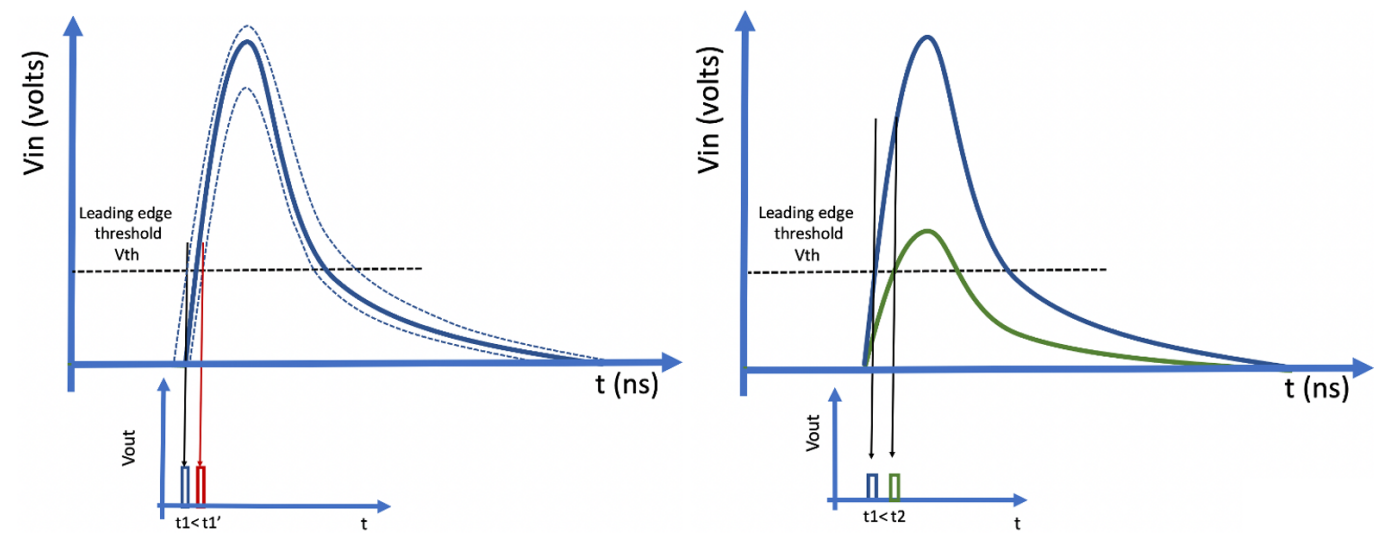

Figure 3.8. Representation of gamma signals as they are processed by means of LEDs. Left: jitter influence. Right: Time-walk influence (variations in the time arrival detection as a function of the amplitude of the incoming signal).

The Leading-Edge Discriminator, is probably the most extended design for the generation of a signal timestamp. Currently, the most advanced readout electronics seen in PET industry are based on these discriminators due to the fact that they are effective and easy to implement even at the microelectronic level (e.g. ASIC). Their operating principle is based on an analog comparator which is set to a fixed threshold. As soon as the incoming pulse crosses this threshold, a logic signal is generated and fed to the next part of the readout chain for the timestamp generation. However, this type of circuitry is highly sensitive to time jitter and time-walk uncertainties. As it can be seen in Figure 3.8 left, random fluctuations might result in the generation of logic pulses at different time frames with respect to the centroid of the pulse. 
Moreover, in Figure 3.8 right, it is observed that the time in which the logic signal is generated, directly depends on the amplitude of the pulse (and rise time). Signals with lower gain, infer a delay in their timestamp determination. Apart from this, although is hard to be appreciated in the following illustration (Figure 3.8 right), this amplitude sensitivity introduces variations in the effective threshold level of the discriminator for the lower amplitude pulses. This occurs due to the fact that when a signal is crossing the threshold, a small additional amount of charge is still needed to actually trigger the discriminator, resulting in additional time delays for slower pulses.

Figure 3.9 demonstrates the impact that the time-walk error might have in terms of timing precision, extracted from one of the papers composing this $\mathrm{PhD}$ work [43]. The 2D counter plots show how the time delay between two signals varies as a function of the energy (amplitude) of the decoded pulse. As it can be appreciated, lower amplitude pulses can result in delays in the range of 2 ns. Hopefully, as we will demonstrate in the main part of this thesis, calibration procedures can be applied and effectively compensate such large influence, and therefore improve the timing determination (see Figure 3.9 right).

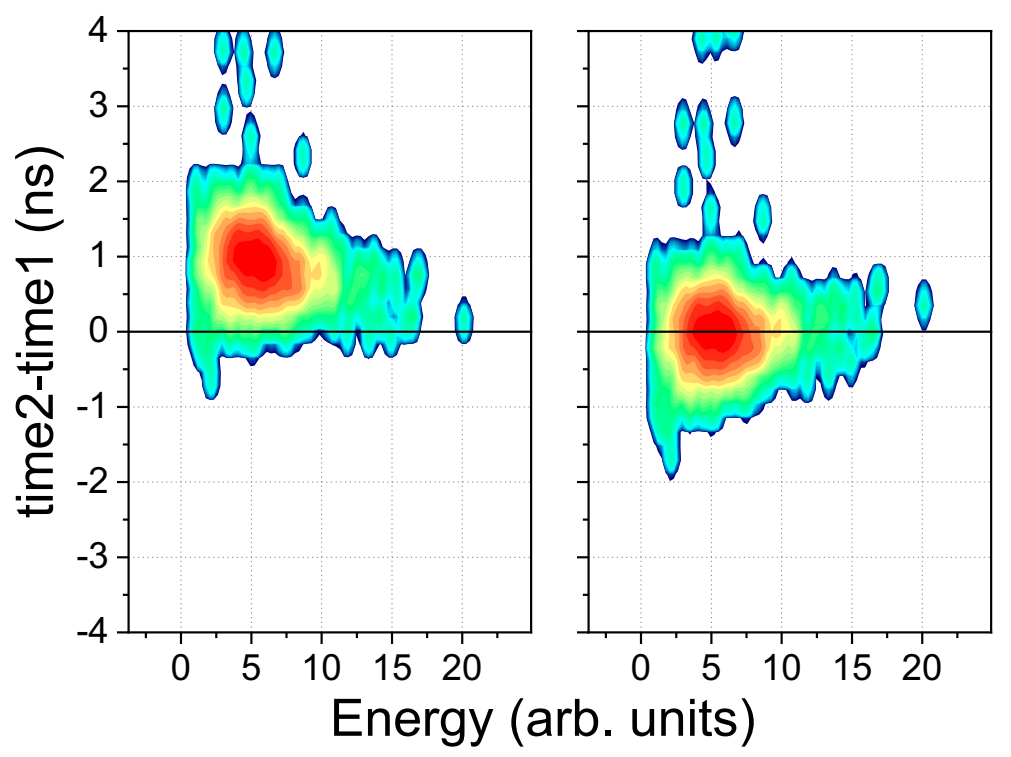

Figure 3.9. Time delay as a function of the pulse energy (amplitude). Left: Before calibration. Right: After calibration.

Some of the challenges encountered when using the LED approach, can be faced if CFD circuitries are applied instead [40][42]. In the operating principle of this type of discriminators, the input signal is split into two branches. One fragment is attenuated to a fraction of the original amplitude, and the other one is delayed and its polarity inverted. These two signals are subsequently summed to form the constant-fraction timing signal. Consequently, the sum of these two signals results in a bipolar pulse with a zero-crossing that corresponds to the original point of optimum fraction on the delayed signal. In the time frame where the bipolar pulse crosses the zero axis, a logical pulse is obtained by means of a discriminator, in order to generate the timestamp. 
Although again some limitations exist, this method is highly efficient as time jitter, amplitude variations and time walk errors are significantly reduced. Therefore, typically better timing precision can be obtained compared to LED based approaches.

Readout electronics based on ASICs are nowadays one of the preferred choices to read and process all signals coming from photosensors and, in particular from SiPMs. Indeed, a highperformance ASIC combines good performance and high applicability, resulting as a good approach for PET detectors evaluation, systems prototyping and even system production. Most of the successful ASIC solutions currently available, integrate leading edge discriminators, operating as threshold triggers, interconnected with Time-To-Digital Converters (TDCs) for the timestamp generation [44]. State-of-the-art TDCs are capable to reach intrinsic resolutions in the range of $30 \mathrm{ps}$, while designs with even better resolution are already being studied [45][46]. Concluding, we can summarize some of the main requirements for TOF-PET readouts as follows:

- Independent signal processing for all available channels

- Accurate energy decoding with linear response

- TDC time resolution below 30 picoseconds

- Configurable thresholds that can be as low as 1 photoelectron

- Double stage threshold schemes to avoid dark count triggering with no dead time

- Baseline correction

- High-rate capabilities

- Low internal capacitance and jitter

\subsubsection{Crystal-Photosensor matching}

The geometrical coupling between crystals and photosensors used in gamma-ray detectors also affects the performance of the timing capabilities of these detectors. This refers to the geometric relation or position of the scintillator with respect to the photosensors [47]. When using pixelated crystals, the most extended approach is the so-called One-To-One coupling (see Figure 3.10 left) [47]. In this approach the scintillation pixels of the crystal arrays precisely match each photosensor active area. In most of the cases, this method is also combined with reflective material covering the surfaces of all crystal elements (except the exit one), in order to increase the optical photons extraction towards the photosensor. Therefore, most of the generated scintillation photons will only be collected by one photosensor, with just few losses to near photosensor elements [38]. This approach is highly effective in terms of timing since a high number of photons results in a sharp rise time (as discussed earlier). However, in some application such as pre-clinical imaging with small animals, some limitations in terms of spatial resolution might appear. Notice that higher spatial resolution requires small crystal sizes leading to a high number of photodetector elements and eventually an increase in the cost and complexity of the system. 
Another crystal-photosensor matching approach makes use of crystal elements with size dimensions smaller than the photosensor active area, but centered (Figure 3.10 center and right). This approach enables achieving optimal timing resolution as there are minimum scintillation photon losses to neighbor photosensors. Moreover, OTTS errors are reduced. As a consequence, the timing resolution is slightly enhanced. Also notice that a system built using this method will suffer from a limited sensitivity due the crystal-to-crystal gaps.

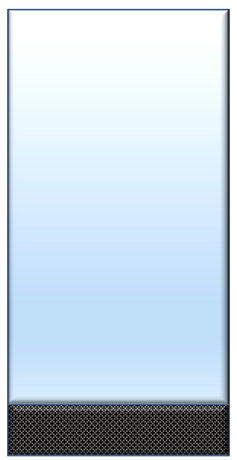

$3 \times 3 \mathrm{~mm}^{2}$ crysta $3 \times 3 \mathrm{~mm}^{2}$ SiPM

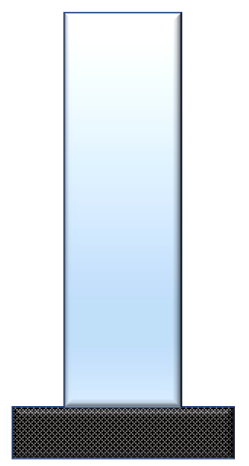

$2 \times 2 \mathrm{~mm}^{2}$ crystal $3 \times 3 \mathrm{~mm}^{2}$ SiPM

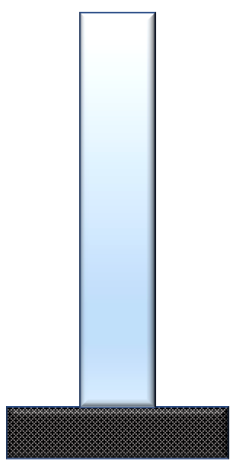

$1 \times 1 \mathrm{~mm}^{2}$ crystal $3 \times 3 \mathrm{~mm}^{2}$ SiPM

Figure 3.10. Coupling of crystals of $3 \times 3,2 \times 2$ and $1 \times 1 \mathrm{~mm}^{2}$ to a SiPM sensor of $3 \times 3 \mathrm{~mm}^{2}$ showing how the light collection can be improved when reducing the crystal dimensions with respect to the SiPM active area. Notice that for large SiPM microcell the design on the right-hand side might suffer from cells saturation.

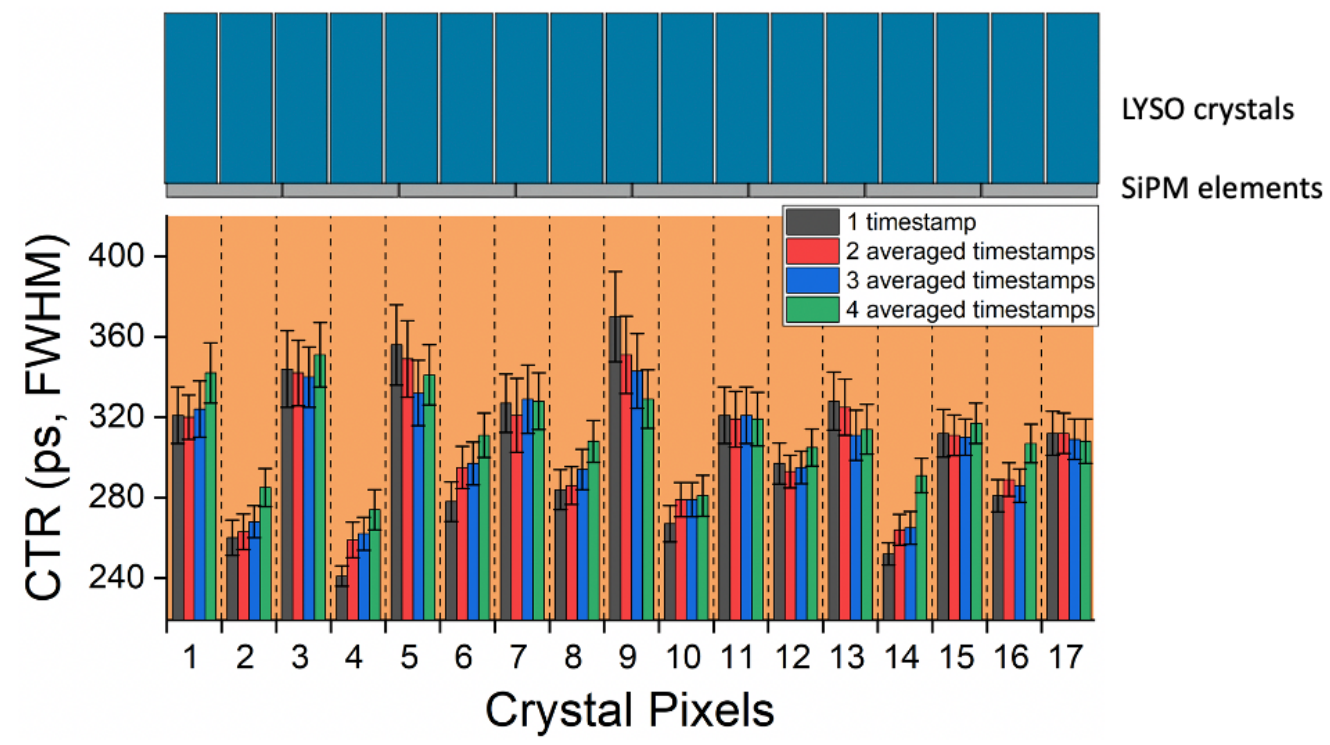

Figure 3.11. Experimental results: Top, relative position of each crystal pixel belonging to one column, with respect to the photosensor elements. Bottom, CTR for each crystal pixel measured with the first timestamp recorded as well as with a weighted averaging method [47].

In order to improve the spatial resolution without significantly increasing the cost and complexity of the system, an alternative coupling method widely used in PET is the so-called light sharing approach [47][48]. In these configurations and, in contrast with the one-to-one 
method, a different number of photodetectors and pixel elements (in the case of pixelated crystals) is used. Notice that the energy and gamma-ray impact position (sometimes including DOI [49]) is obtained by combining the information from all involved photosensor elements. This means all elements receiving scintillation photons above certain threshold. Depending on the exact position of the crystal element with respect to the SiPM array, different light distributions, and consequently light collections by the SiPMs are found. As it will be in detail discussed in Chapter 4, although this approach typically permits good spatial resolution, it challenges the timing determination of the event [43]. As an example, Figure 3.11 depicts an experiment carried out in the framework of this doctoral study, in which a crystal array of $17 \times 17$ elements (each one of $\left.1.5 \times 1.5 \times 10 \mathrm{~mm}^{3}\right)$ coupled to a SiPM array of $8 \times 8$ elements $\left(3 \times 3 \mathrm{~mm}^{2}\right)$ is tested. The aim was to shed light to the time resolution dependency with crystal-photosensor element position. As it can be observed in the figure, the time resolution is affected by light losses occurred due to the crystal pixel-SiPM misalignment [47].

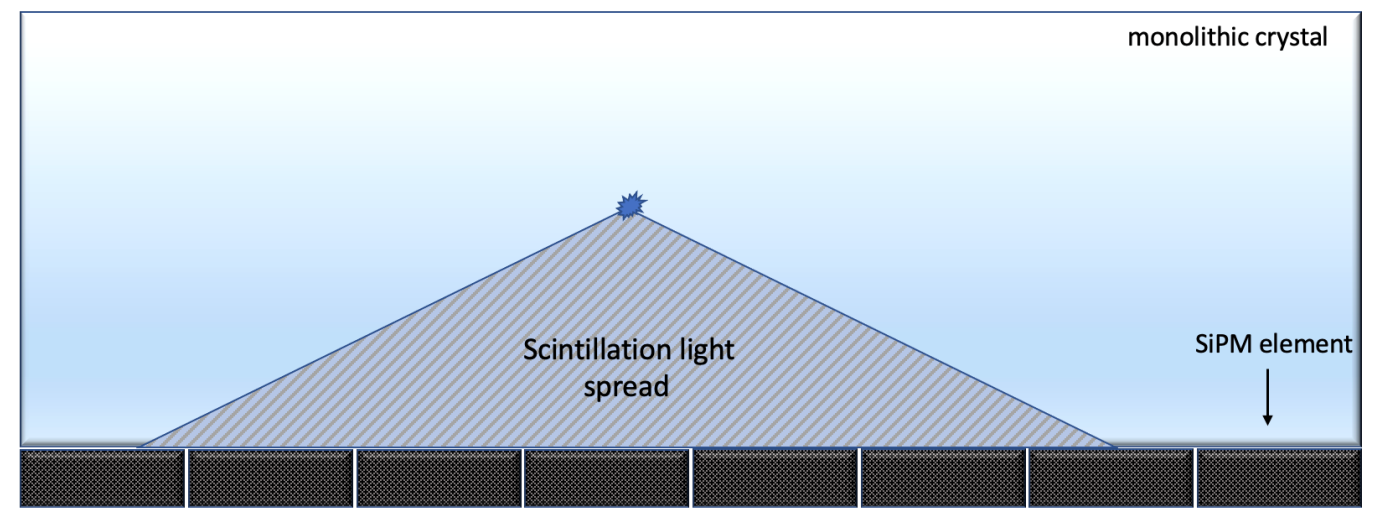

Figure 3.12. 2D representation of light sharing detector configuration. Top: Light sharing based on crystal arrays. Bottom: Detector configuration based on monolithic scintillator.

Light sharing approaches also refer to the use of monolithic or semi-monolithic crystal geometries, as illustrated in Figure 3.12. Monolithic scintillators are nowadays very attractive due to several advantages. In terms of spatial resolution, they are not limited by the pixel size, allowing one to reach sub-millimeter intrinsic resolution capabilities [50][53]. As it was mentioned before, monolithic scintillators also provide access to the gamma-ray DOI information, improving the system performance at the FOV edges of PET systems. This occurs due to the fact that the scintillation photons can travel with almost no reflections (besides the one in the lateral and entrance walls when no absorbent treatments applied) generating a light distribution. Although this light distribution can highly benefit system spatial resolution [51]-[53], can severely impact in the event timing determination [50]. The reason simply relies on the fact that the generated visible photons are shared among several photosensor elements; in direct contrast with the case of the one-to-one coupling in pixelated crystals, in which most of the scintillation light is captured by one photosensor. Consequently, in a monolithic based detector, a significant number of readout channels is typically triggered while the channels lack of input signals with 
sharp rising edges and enough amplitude. In other words, each readout channel shows a poor SNR which makes the time decoding highly challenging. Time-walk, jitter and false triggering are the main factors that affect the process of timing.
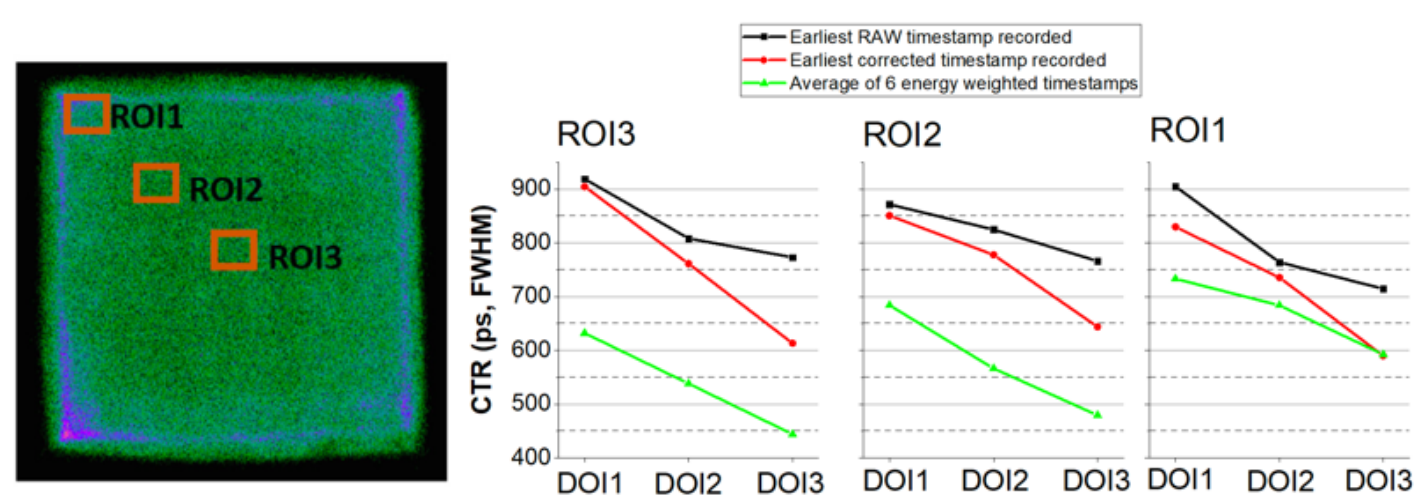

Figure 3.13. Left: flood map of events in the monolithic block, $15 \mathrm{~mm}$ thick and $50 \mathrm{~mm}$ size (square), showing the three ROI selected for analysis. Right: Time resolution dependency with the gamma event position, as determined following a series of calibrations and post-processing methods [43].

Aiming to better illustrate this effect, in the Figure 3.13 left we present an experimental evaluation. We observed, for gamma events occurring near the entrance of the crystal (DOI1), the number of the triggered channels is above 30 (see section 4 for more details). In addition to this, we estimated that in the same DOI1 region, even the readout channel which collected the highest amount of energy (charge) inside a given gamma event, it did not collect more than a fraction of $10 \%$ of the whole amount generated during the scintillation process. These effects are directly reflected in the CTR capabilities of this detector set-up (Figure 3.13 right). As it will be further discussed during the main part of thesis, there is a CTR dependency with the data calibration and number of time-stamps used for such determination. Moreover, the Volume-Of-Interest (VOI) considered, defined by the DOI and Region-of-Interest (ROI), also impacts the reached CTR. It can be appreciated that determining an accurate timing resolution for this type of monolithicbased detector is challenging. However, in several cases, specific approaches, calibrations and post-processing methods might result highly efficient and can permit a significant improvement. 


\section{References}

[1] M. M. Ter-Pogossian et al., Photon time-of-flight-assisted positron emission tomography. Journal of Computer Assisted Tomography. 5 (1981), 227-239.

[2] M. M. Ter-Pogossian et al., Super PETT I: A Positron Emission Tomograph Utilizing Photon Time-of-Flight Information. IEEE Transactions on Medical Imaging. 1 (1982), 179-187.

[3] D. C. Ficke et al., TOF Acquisition: System Design and Experimental Results. Proceedings of the Workshop on Time-of-Flight Tomography. 453 (1982), 139-141.

[4] D. C. Ficke, D. E. Beecher, G. R. Hoffman, T. J. Holmes, M. M. Ter-Pogossian, Recent developments in image reconstruction using a time-of-flight-assisted positron emission tomograph: Super PETT I. IEEE Transactions on Nuclear Science. 31 (1984), 605-608.

[5] T. J. Holmes, R. E. Hitchens, G. J. Blaine, A dedicated hardware architecture for data acquisition and processing in a time-of-flight emission tomography system (SuperPETT). IEEE Transactions on Nuclear Science. 30 (1983), 170-174.

[6] M. Conti, Focus on time-of-flight PET: The benefits of improved time resolution. European Journal of Nuclear Medicine and Molecular Imaging. 38 (2011), 1147-1157.

[7] S. Surti et al., Impact of time-of-flight PET on whole-body oncologic studies: A human observer lesion detection and localization study. Journal of Nuclear Medicine, 52 (2011), $712-719$.

[8] M. Conti, State of the art and challenges of time-of-flight PET. Physica Medica, 25 (2009), 1-11.

[9] S. Vandenberge et al., Recent developments in time-of-flight PET. EJNMMI Physics. 3:1 (2016), 3

[10] M. Conti and B. Bendriem, The new opportunities for high time resolution clinical TOF PET. Clinical and Translational Imaging. 7 (2019), 139-147.

[11] P. Lecoq et al., Roadmap toward the 10 ps time-of-flight PET challenge, Physics in Medicine and Biology. In press, 2020.

[12] P. Lecoq, Pushing the Limits in Time-of-Flight PET Imaging. IEEE Transactions on Radiation and Plasma Medical Sciences. 1 (2017), 473-485.

[13] S. Surti, Update on time-of-flight PET imaging. Journal of Nuclear Medicine. 56 (2015), 98-105.

[14] S. R. Cherry et al., Total-body PET: Maximizing sensitivity to create new opportunities for clinical research and patient care. Journal of Nuclear Medicine. 59 (2018), 3-12. 
[15] E. Lamprou et al., CardioPET, A high resolution TOF-PET for Limited Angle Tomography. IEEE Nuclear Science Symposium 2020.

[16] S. Surti et al., Performance of Philips Gemini TF PET/CT scanner with special consideration for its time-of-flight imaging capabilities. Journal of Nuclear Medicine. 48 (2007), 471-480.

[17] V. Bettinardi et al., Physical Performance of the new hybrid PETCT Discovery-690. Medical Physics. 38 (2011), 5394-5411.

[18] J. B. W. Jakoby et al., Physical and clinical performance of the mCT time-of-flight PET/CT scanner. Physics in Medicine and Biology. 56 (2011), 2375-2389.

[19] T. K. Lewellen, Recent developments in PET detector technology. Physics in Medicine and Biology. 53 (2008), R287-R317.

[20] D. Henseler et al., SiPM performance in PET applications: An experimental and theoretical analysis. IEEE Nuclear Science Symposium Conference Record (2009), 1941-1948.

[21] B. Dolgoshein et al., Status report on silicon photomultiplier development and its applications. Nucl. Instrum. Methods A. 563 (2006), 368-376.

[22] C. L. Kim, G. C. Wang, S. Dolinsky, Multi-pixel photon counters for TOF PET detector and its challenges. IEEE Transactions on Nuclear Science. 56 (2009), 2580-2585.

[23] N. C. Nguyen et al., Image quality and diagnostic performance of a digital pet prototype in patients with oncologic diseases: Initial experience and comparison with analog PET. Journal of Nuclear Medicine. 56 (2015), 1378-1385.

[24] [I. Rausch et al., Performance evaluation of the VereoS PET/CT system according to the NEMA NU2-2012 standard. Journal of Nuclear Medicine. 60 (2019), 561-567.

[25] D. F. C. Hsu et al., Studies of a next-generation silicon-photomultiplier-based time-offlight PET/CT system. Journal of Nuclear Medicine. 58 (2017), 1511-1518

[26] J. S. Reddin et al., Performance evaluation of the SiPM based Siemens Biograph vision PET/CT system. 2018 IEEE Nuclear Science Symposium and Medical Imaging Conference, NSS/MIC 2018 Proceedings.

[27] J. Van Sluis et al., Performance characteristics of the digital biograph vision PET/CT system. Journal of Nuclear Medicine. 60 (2019), 1031-1036.

[28] D. R. Schaart et al., LaBr3:Ce and SiPMs for time-of-flight PET: Achieving 100 ps coincidence resolving time. Physics in Medicine and Biology. 55 (2010), 179-189.

[29] J. W. Cates, S. Gundacker, E. Auffray, P. Lecoq, C. S. Levin, Improved single photon time resolution for analog SiPMs with front end readout that reduces influence of electronic noise. Physics in Medicine and Biology. 63 (2018), 185022. 
[30] S. Gundacker et al., Time of flight positron emission tomography towards 100ps resolution with L(Y)SO: An experimental and theoretical analysis. Journal of Instrumentation. 8 (2013), P07014.

[31] S. E. Derenzo, W. S. Choong, W. W. Moses, Fundamental limits of scintillation detector timing precision. Physics in Medicine and Biology. 59 (2014), 3261-3286.

[32] S. Vinogradov, Approximations of coincidence time resolution models of scintillator detectors with leading edge discriminator. Nucl. Instrum. Methods Phys. Res. A. 912 (2018), 149-153.

[33] S. Gundacker et al., Time resolution deterioration with increasing crystal length in a TOFPET system. Nucl. Instrum. Methods Phys. Res. A. 737 (2014), 92-100.

[34] A. Wirrwar et al., Influence of crystal geometry and wall reflectivity on scintillation photon yield and energy resolution, 1999 IEEE Nuclear Science Symposium. Conference Record. 3 (1999), 1443-1445.

[35] F. Acerbi, S. Gundacker, Understanding and simulating SiPMs. Nucl. Instrum. Methods Phys. Res. A. 926 (2019), 16-35.

[36] S. Gundacker, A. Heering, The silicon photomultiplier: fundamentals and applications of a modern solid-state photon detector. Physics in Medicine and Biology. 65 (2020), 17TR01.

[37] S. Gundacker, E. Auffray, P. Jarron, T. Meyer, P. Lecoq, On the comparison of analog and digital SiPM readout in terms of expected timing performance. Nucl. Instrum. Methods Phys. Res. A. 787 (2015), 6-11.

[38] S. Gundacker et al., Experimental time resolution limits of modern SiPMs and TOF-PET detectors exploring different scintillators and Cherenkov emission. Physics in Medicine and Biology. 65 (2020), 025001

[39] M. W. Fishburn, E. Charbon, System tradeoffs in gamma-ray detection utilizing SPAD arrays and scintillators. IEEE Transactions on Nuclear Science. 57 (2010), 2549-2557.

[40] G. F. Knoll, H. W. Kraner, Radiation Detection and Measurement. Proceedings of the IEEE. 69 (1981), 495.

[41] T. J. Paulus, Timing electronics and fast timing methods with scintillation detectors. IEEE Transactions on Nuclear Science. 32 (1985), 1242-1249.

[42] ORTEC, Model 935 Data Sheet, [Online]. Available: http://www. ortec-online.com.

[43] E. Lamprou et al., Exploring TOF capabilities of PET detector blocks based on large monolithic crystals and analog SiPMs. Physica Medica. 70 (2020), 10-18.

[44] S. Surti, J. S. Karp, Advances in time-of-flight PET. Physica Medica. 32 (2016), 12-22. 
[45] M. W. Fishburn, E. Charbon, Time-to-digital converters for PET: An examination of metrology aspects. IEEE Nuclear Science Symposium Conference Record (2012), 839840.

[46] T. Sui et al., Pico-TDC: A novel FPGA-based TDC with 2.2ps RMS timing resolution. 2017 IEEE Nuclear Science Symposium and Medical Imaging Conference, NSS/MIC 2017 - Conference Proceedings (2017).

[47] E. Lamprou et al., In-depth evaluation of TOF-PET detectors based on crystal arrays and the TOFPET2 ASIC. Nucl. Instrum. Methods Phys. Res. A. 977 (2020), 164295

[48] R. Marcinkowski, S. España, R. Van Holen, S. Vandenberghe, Optimized light sharing for high-resolution TOF PET detector based on digital silicon photomultipliers. Physics in Medicine and Biology. 59 (2014), 7125-7139.

[49] M. Pizzichemi et al., A new method for depth of interaction determination in PET detectors. Physics in Medicine and Biology. 61 (2016), 4679-4698.

[50] E. Lamprou et al., PET detector block with accurate 4D capabilities. Nucl. Instrum. Methods Phys. Res. A. 912 (2018), 132-136.

[51] A. Gonzalez-Montoro et al., Performance study of a large monolithic LYSO PET detector with accurate photon DOI using retroreflector layers. IEEE Transactions on Radiation and Plasma Medical Sciences. 1 (2017), 229-237.

[52] A. González-Montoro et al., Novel method to measure the intrinsic spatial resolution in PET detectors based on monolithic crystals. Nucl. Instrum. Methods Phys. Res. A. 920 (2019), 58-67.

[53] S. Krishnamoorthy et al., Performance evaluation of the MOLECUBES $\beta$-CUBE - A high spatial resolution and high sensitivity small animal PET scanner utilizing monolithic LYSO scintillation detectors. Physics in Medicine and Biology. 63 (2018), 155013. 


\section{Objectives}

Following this introduction, we present here the objectives of novel high-performance PET developments. As we discussed, the benefits risen from the combination of a good spatial and timing resolution are essential for the modern clinical diagnostic practice. However, these characteristics have to be combined in an efficient way, both from the development but also from the clinical point of view, meaning that a trade-off between performance, cost, complexity and accessibility has to be reached.

Driven by these aims, under the framework of this $\mathrm{PhD}$, we propose innovative detector concepts, some of them studied here for the first time. They, will infer all desired characteristics and will become outstanding candidates for both clinical and pre-clinical (small animal imaging) applications. In the following chapters, through a series of published works, we will demonstrate the benefits related with the proposed configurations, while emphasis will be given to the challenges that appeared and, in the steps, required to deal with them. In the following paragraphs, and before proceeding to the main part of this thesis, we briefly introduce our objectives.

First, we attempted to develop PET detector solutions, capable of accurately decoding each annihilation event in all three spatial coordinates as well as to provide accurate timing information. Originally, the best candidate for that kind of configuration is a monolithic crystal. Indeed, the intrinsic benefits of monolithic blocks in terms of spatial resolution and sensitivity, as well as their typically lower cost compared to other configurations (e.g. crystal arrays) make them a highly attractive solution. However, in monolithic based detectors, challenges appear in the timing determination of the annihilation event. According to what was described earlier in chapter 3 , the intense light sharing that occurs inside a monolithic crystal, challenges some key TOF requirements, meaning that a poor timing performance is highly probable. However, as we will demonstrate, these limitations can be partially overcome, and through specific methods and approaches, the time precision can be improved. More in detail, in sections 4.1, 4.2 and 4.3, monolithic crystals are being evaluated, while the main emphasis has been given to their timing capabilities. To our knowledge, this is the very first study, in which analog SiPMs are combined with monolithic crystals and high-end electronics (ASICs) for TOF-PET applications. One of the final aims when accomplishing this study, is to make use of the proposed detector configurations in PET scanners with limited angle tomography geometry.

Another research line followed in the framework of this $\mathrm{PhD}$ thesis, is focusing in the evaluation of detector configurations based on novel crystal arrays. The so-called One-To-One coupling approach was evaluated in depth. Moreover, special interest is given to light sharing configurations with crystal arrays. This is a well-studied method, but mainly is oriented towards an improved spatial resolution, since it typically lacks of accurate timing due to this light sharing effect among multiple photosensors. However, motivated by the known benefits of a good timing 
resolution, we have extensively explored also their TOF performance in a quite novel way, aiming to shed light on the factors that essentially limit their timing capabilities, and will consist a useful reference for future works. This study is detailed in section 4.4.

All experiments described in this thesis have been carried out using analog SiPMs of different types and sizes, while the read-out task was performed using commercially available ASIC-based readouts. In particular, the first study (4.1) was carried out using the TOFPET ASIC (PETsys Electronics, Lisbon) while in the following ones, this readout was upgraded to TOFPET2. This chip, was chosen as it showed good performance, while it could read out and process up to hundreds of thousands of channels, making it also suitable for PET prototypes and proof-ofconcepts development. A more detailed description of the ASIC and design scheme, can be found in the following contributions. 


\section{Chapter 4}

\section{Contributions}

Forming new ideas, creating concepts and providing solutions to existing problems, are basic ingredients of the research concept. Probably, it is not an exaggeration to state that research consists one of the main pathways towards innovations and breakthroughs, which can directly impact both present and future. As long as researchers and engineers are driven by inspiring motivations, great discoveries and advances can be made, literarily transforming the world as we know it.

\subsection{PET detector block with accurate 4D capabilities}

This first paper included, describes our pilot efforts towards introducing monolithic scintillators in TOF-PET detectors. The reader can find in this contribution also some initial results when using crystal arrays of small pixels $(1.6 \mathrm{~mm})$. This configuration allowed us to more accurately determine possible limitations in the experimental set-up, as well as limits in our proposed concept.

More in detail, we carried out all experiments using an ASIC-based readout. This particular ASIC was initially presented in 2013 as a promising readout electronics for PET. We selected a pair of SiPM arrays of SiPM with $8 \times 8$ elements with an active area of $6 \times 6 \mathrm{~mm}^{2}$ each. Having as a reference the same SiPM photosensors and readout method, we then proceeded to a detailed comparison between the crystal array $(32 \times 32$ pixels, $1.5 \times 1.5 \mathrm{~mm}$ each $)$ and a monolithic block $\left(50 \times 50 \times 15 \mathrm{~mm}^{3}\right)$. In a series of coincidence measurements, both spatial and timing resolution is evaluated for the two detector set-ups.

Using the scintillation block based on the crystal array, a detector spatial resolution in the range of $1.5 \mathrm{~mm}$ is eventually achieved, which this was combined with an average time resolution for all 64 channel pairs of 553 ps FWHM. In the same direction, the detector configuration based on the monolithic crystal, provided a still moderated spatial resolution nearing $2.6 \pm 0.5 \mathrm{~mm}$ while the CTR was determined at $1.2 \mathrm{~ns}$ after correcting for the time walk-error.

Although most of the results were slightly poorer than expected, this preliminary study allowed us to design, develop and improve specific strategies to enhance detector performance. 
Additionally, following these experiments, we were able to determine and better estimate the limitations of the read-out part. This specific chip has gone through few versions. The first version used here lacked of capabilities to accurately decode each signal coming from the photosensors, impacting the measured resolutions. However, following versions of this read-out exhibited improved performance, as it will be shown to upcoming contributions. 


\title{
PET detector block with accurate 4D capabilities
}

Efthymios Lamprou, Albert Aguilar, Andrea González-Montoro, Jose M. Monzó, Gabriel Cañizares, Sofia Iranzo, Luis F. Vidal, Liczandro Hernández, Pablo Conde, Sebastian Sánchez, Filomeno Sánchez, Antonio J. González and José M. Benlloch

Published: Nuclear Instruments and Methods in Physics Research A, Volume 912, Pages 132-136, 2018.

DOI: https://doi.org/10.1016/j.nima.2017.11.002

\begin{abstract}
In this contribution, large SiPM arrays $\left(8 \times 8\right.$ elements of $6 \times 6 \mathrm{~mm}^{2}$ each) are processed with an ASIC-based readout and coupled to a monolithic LYSO crystal to explore their potential use for TOF-PET applications. The aim of this work is to study the integration of this technology in the development of clinical PET systems reaching sub-300 ps coincidence resolving time (CRT). The SiPM and readout electronics have been evaluated first, using a small size $1.6 \mathrm{~mm}(6 \mathrm{~mm}$ height $)$ crystal array $(32 \times 32$ elements $)$. All pixels were well resolved and they exhibited an energy resolution of about $20 \%$ (using Time-over-Threshold methods) for the $511 \mathrm{keV}$ photons. Several parameters have been scanned to achieve the optimum readout system performance, obtaining a CRT as good as $330 \pm 5$ ps FWHM. When using a black-painted monolithic block, the spatial resolution was measured to be on average $2.6 \pm 0.5 \mathrm{~mm}$, without correcting for the source size. Energy resolution appears to be slightly above 20\%. CRT measurements with the monolithic crystal detector were also carried out. Preliminary results as well as calibration methods specifically designed to improve timing performance, are being analyzed in the present manuscript.
\end{abstract}

\section{Introduction}

Whole body PET scanners exhibit some limitations when imaging small organs or lesions. The concept of dedicated PET systems offers a variety of advantages in the clinical practice. In some cases, these systems follow geometries different from fully closed rings. Fig. 1 depicts, as an example, a possible implementation for a cardiac PET under stress situation. The missing angular information, when using two panels geometries, may introduce artifacts visible in the reconstructed images across the Field-of-View, worsening the final system performance [1]. However, it has been shown that accurate information on the detected photons timing (Time-ofFlight, TOF) can significantly reduce these effects [2].

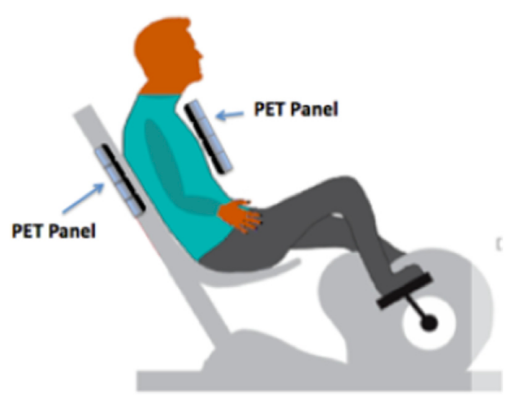

Fig. 1. Sketch of a heart PET with limited angle tomography geometry, where the patient is in a stress situation while cycling. 
Our proposal consists of using high resolution PET detectors based on monolithic scintillators capable to provide precise TOF. Monolithic scintillators blocks allow one for the accurate determination of the three-dimensional photon impact coordinates, as they typically preserve the scintillation light distribution profile. In addition, we suggest using Application Specific Integrated Circuits (ASIC) readout allowing us to independently read each SiPM pixel and making it possible to achieve optimal TOF performance. All together, 3D spatial and timing resolution, is what we dubbed 4D capabilities.

Building PET detector blocks based on monolithic scintillators crystals infer some challenges. First, a wider scintillation light distribution compared to crystal arrays, results in a high number of SiPM pixels that are hit per photon impact, affecting the signal-to-noise-ratio (SNR). The 4D impact determination is also affected by the increase in dark counts arising from the photosensors. Moreover, strong influence by the time walk error is being observed as a result of the low SNR. For the current case of light sharing among several SiPMs, the timestamp of the event is deduced from averaging methods among the known individual timestamps [3]. In this work, we present calibration methods designed to overcome these limitations. Experimental results both with monolithic and pixelated scintillator crystals coupled to SiPMs, are presented and evaluated. Promising results are obtained in terms of energy, spatial and timing resolution.

\section{Materials and Method}

\subsection{ASIC readout}

Reading and digitizing the signals coming from the SiPM photosensors is carried out using the ASIC chip TOFPET1 from PETsys (Lisbon, Portugal). The input stage of the ASIC is composed of dedicated amplifiers, discriminators and Time-to-Digital Converters (TDCs) [4]. The charge is provided using the Time-over-Threshold (ToT) method, which measures the time in which a signal stays above a predefined threshold. Information on all channels is managed by an intermediate board, which packs the information of all ASICs into a single data frame $(72 \times$ 106 events/s output rate) and sends it to the DAQ board ( $250 \times 106$ events/s output rate), connected to a PC via PCI-e link. Fig. 2 shows the photograph of the SiPM array on the left, and the detector block including the ASIC and an adaptor board on the right.

Aiming to build detectors suitable for clinical applications, a large photosensor area is demanded. The photosensors used throughout all the measurements were the SensL J-series $8 \times 8$ SiPM arrays with $6 \times 6 \mathrm{~mm}^{2}$ active area per SiPM and pitch of $6.33 \mathrm{~mm}$. This array exhibits low dark count rate $\left(30 \mathrm{kHz} / \mathrm{mm}^{2}\right.$ at the breakdown voltage), uniform bias voltage and low temperature drift. This makes it suitable for applications with monolithic blocks in which the SNR is relatively small. 


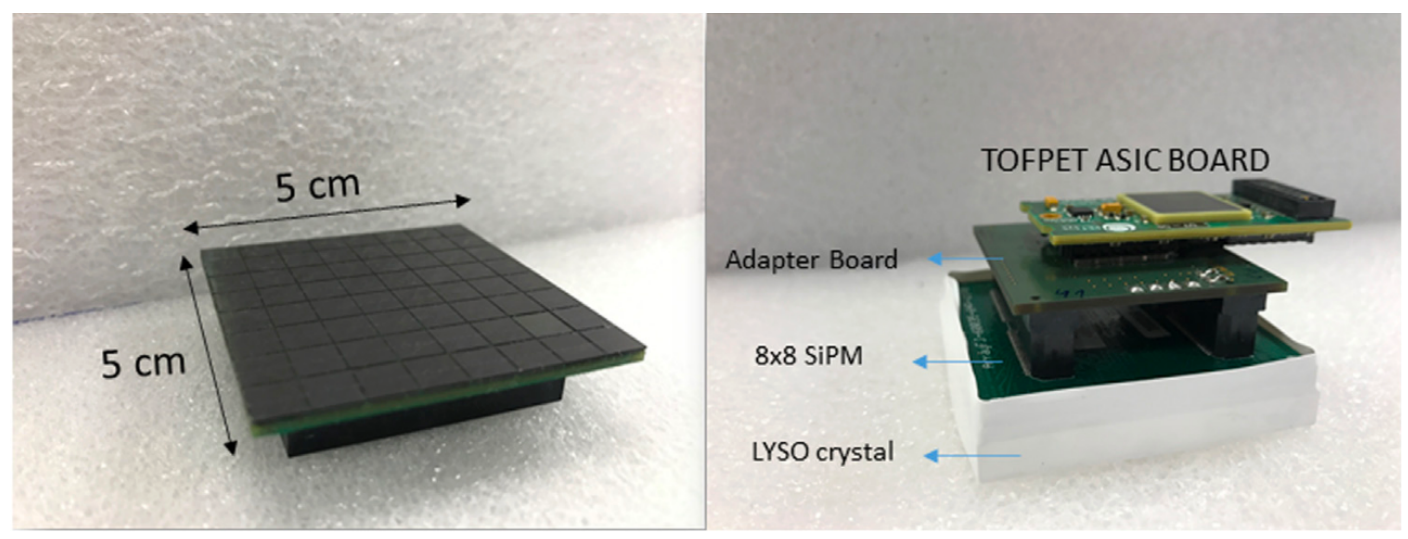

Fig. 2. Left. $8 \times 8 \mathrm{SiPM}$ array with $6.33 \mathrm{~mm}$ pitch. Right: detector block composed of LYSO crystal, $8 \times 8 \mathrm{SiPMs}$ array, an adaptor board and the FEB-A ASIC board.

\subsection{Set-up}

Experiments where initially performed using LYSO crystal arrays of roughly $50 \mathrm{~mm} \times 50$ $\mathrm{mm} \times 6 \mathrm{~mm}$ and $1.6 \mathrm{~mm}$ pixel size. This setup facilitates the evaluation of the electronics performance channel by channel. By scanning several SiPM pairs from opposite photo-detectors, the system capability to determine CRT was precisely evaluated. We set a controlled temperature environment near $20{ }^{\circ} \mathrm{C}$.

In addition to pixelated crystals, we also carried out tests using a monolithic LYSO scintillator with trapezoidal shape $(50 \mathrm{~mm} \times 50 \mathrm{~mm}$ exit face, $40 \mathrm{~mm} \times 40 \mathrm{~mm}$ entrance face, and $10 \mathrm{~mm}$ thickness) with entrance and lateral surfaces black painted. A monolithic block with small thickness was chosen for this pilot evaluation. We characterized the detector block in terms of energy and spatial resolution, by carrying out coincidence measurements with a reference detector based on the above crystal array (50 $\mathrm{cm}$ of separation between detectors). Concerning the timing capabilities of the monolithic block, they were explored using a single $6 \mathrm{~mm} \times 6 \mathrm{~mm} \times 15 \mathrm{~mm}$ LYSO pixel coupled to one SiPM as a reference detector. The distance between the two detectors was reduced to $20.5 \mathrm{~cm}$, increasing the count rate.

\section{Results}

\subsection{Crystal arrays}

The detector block under study shows the capability to resolve crystal elements as small as $1.6 \mathrm{~mm}$, as depicted in Fig. 3. We have computed the boundary regions for each crystal pixel, so-called Voronoi diagrams [5]. The $32 \times 32$ pixels/regions are clearly distinguished, as depicted in Fig. 4. Energy resolution for single pixels was measured to be nearing $20 \%$ before ToT calibration. 

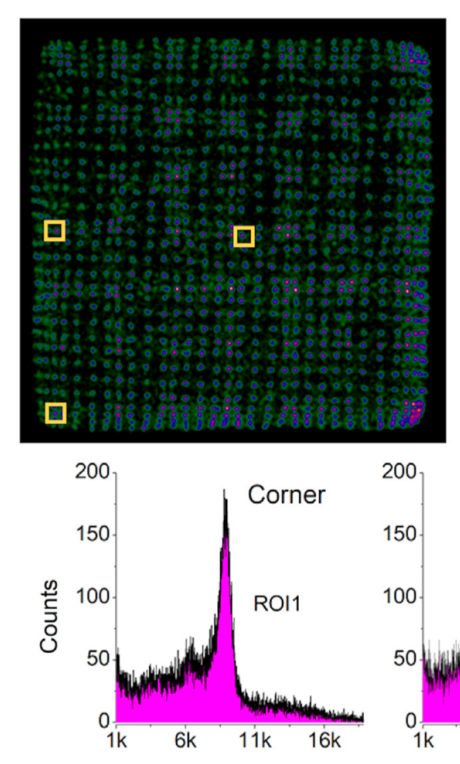

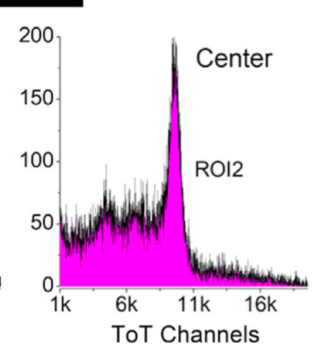

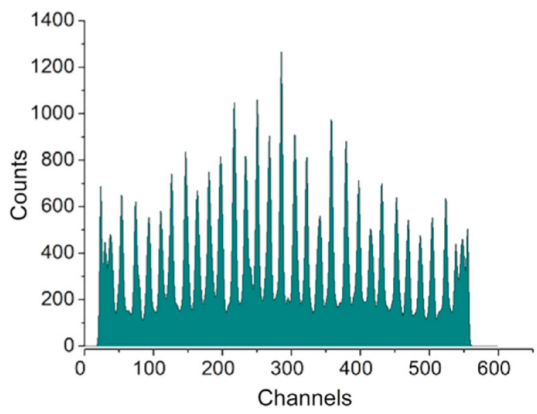

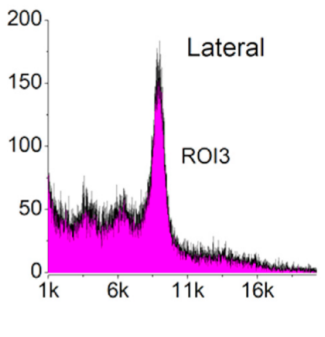

Fig. 3. Top-left: flood map of the $1.6 \mathrm{~mm}$ pixels for the whole detector. Top-right: a detail of an $X$ projection. Bottom: energy spectra for three different small regions of interest (ROI) marked with yellow boxes in the flood map, namely at the corner, center and lateral areas.

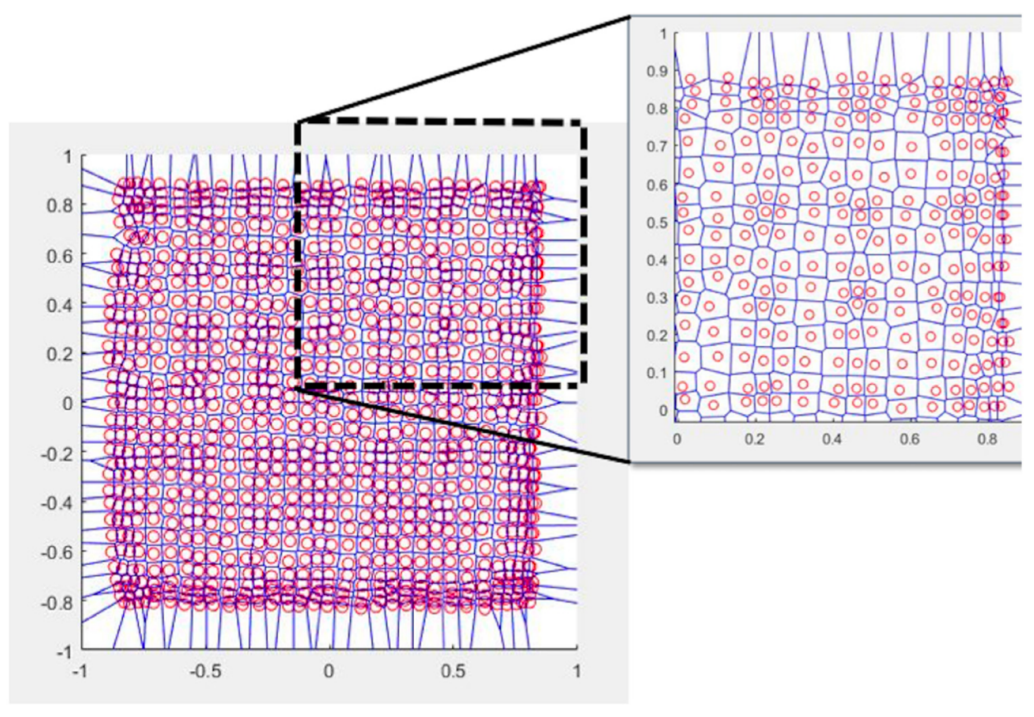

Fig. 4. Voronoi diagrams of a measurement performed with a LYSO crystal array (pixel size $1.6 \mathrm{~mm}$ ).

For CRT measurements, a calibration of ASIC internal TDCs and Operation Amplifiers (OAs) was performed. We obtained the optimal ASIC configuration regarding CRT, by scanning the SiPM bias voltage [26-31 V], the two OA parameters named ib1 and vbl (which are current and voltage polarization parameters, in DAC units), and the time threshold. Fig. 5 shows the CRT measurements as a function of these parameters. Best CRT values were found for bias voltages nearing $29.5 \mathrm{~V}$. Optimum average vbl and ib1 parameters were measured to be 45 and 15 , respectively. Fig. 5 top only shows some examples. The optimal configuration for the time threshold was determined nearing 14 DAC units above the baseline at the SiPM bias voltage of 29.5 V. Afterwards, CRT measurements for direct channel pairs (opposite SiPMs, 64 in total) 
were recorded as a good sample of all possible pairs obtaining the best value at $330 \pm 5$ ps FWHM (mean value 553 ps, standard deviation 114 ps), see panel bottom-right. Notice that these results were obtained without compensating the time alignment among different channels.
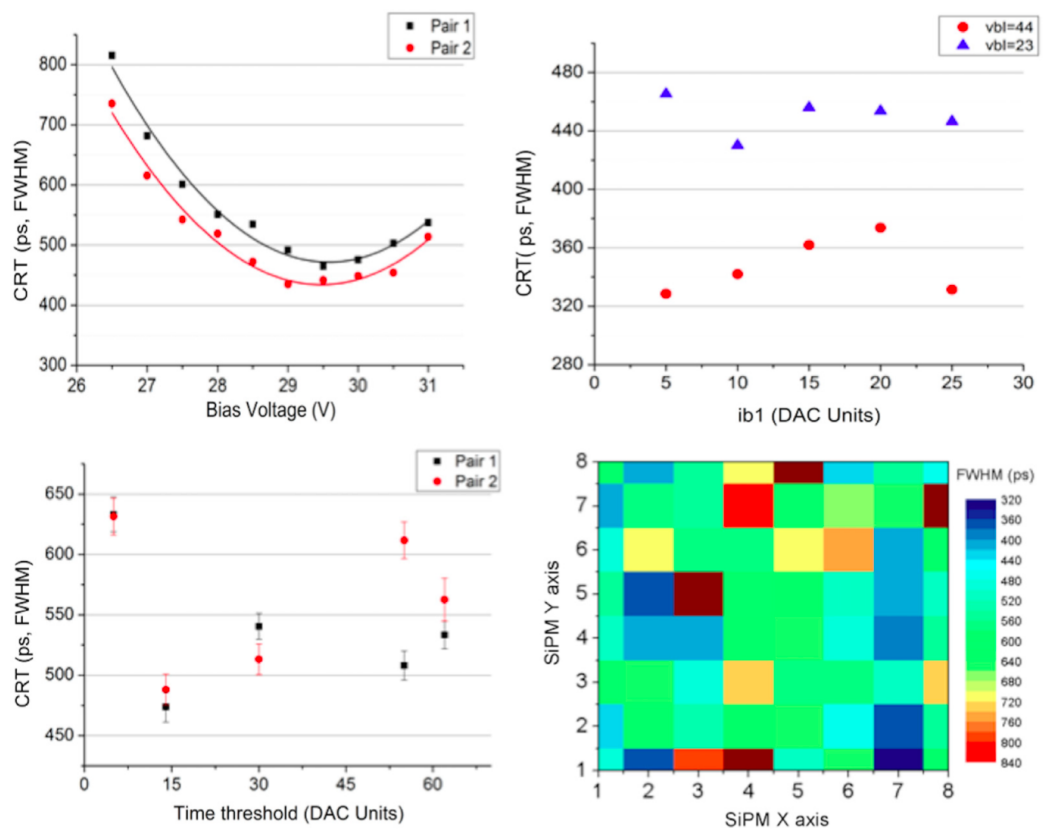

Fig. 5. Top-left: CRT as a function of SiPM bias voltage for two different SiPM pairs. Top-right: CRT as a function of current polarization parameter ib1 for two vbl values. Bottom-left, CRT as a function of trigger threshold for two SiPM pairs. Bottom-right: CRT associated to each SiPM direct pair with the optimal OA parameters.

\subsection{Monolithic blocks}

The capabilities of the detector using a monolithic LYSO crystal were characterized by performing measurements in coincidence. $\mathrm{A}^{22} \mathrm{Na}$ array composed of $11 \times 11$ sources $(1 \mathrm{~mm}$ in diameter, $4.6 \mathrm{~mm}$ pitch, total activity near $20 \mu \mathrm{Ci}$ ) was attached to the entrance face of the scintillation block and data were recorded for about $5 \mathrm{~min}$. The system spatial resolution was evaluated after considering only line of responses (LORs) with angles smaller than $1.43^{\circ}$ from the normal. Then by applying Center of Gravity ( $\mathrm{CoG}$ ) calculation the photon impact position is determined. As depicted in the flood image in Fig. 6 top-left, it was possible to resolve the $9 \times 9$ central sources. By fitting a multi- Gaussian distribution across the profile of one row of sources, an average FWHM of $2.6 \pm 0.5 \mathrm{~mm}$ was obtained. It should be noticed, that this is a preliminary estimation and further improvement is expected after using more accurate mechanical alignment, other types of mechanical collimation as well as another impact algorithm estimator (see Fig. 6 bottom). Finally, an energy resolution for a single source nearing $24 \%$ was estimated.

The timing capabilities of the above set-up were explored after applying a calibration process to sequentially compensate both the time-walk error and the uncertainly introduced by the different time paths among SiPMs channels (time alignment) [3]. Coincidence measurements were carried out between the reference pixel detector and the monolithic block. For all the 64 channel pairs, 
we determined the CRT as a function of ToT values (Fig. 7 left). We took CRT projections of the 2D plot (Fig. 7 left) as a function of small ToT ranges. Fig. 7 left depicts 3 examples of CRT distributions. For all those profiles we calculated the centroid and FWHM using Gaussian fits. We forced time differences to be equal to zero (assume the source is on the middle of the detectors). We carried out this procedure for all 64 channels. Thus, we compensated the unwanted effects, time walk (for a single channel) and time alignment among all channels. With this information we built a table for each channel pair with the mean ToT value, the Gaussian centroid and sigma. Thus, each measurement was time walk and alignment corrected as a function of the channel pair and ToT. As plotted in Fig. 7 right, the CRT distribution after calibration is now centered at zero showing a narrower uncertainty (FWHM), except for low ToT values. We have measured a CRT value of 1.2 ns FWHM by considering only the timestamps with the highest number of photons per impact and filtering at the $511 \mathrm{keV}$ photopeak in both coincidence detectors.
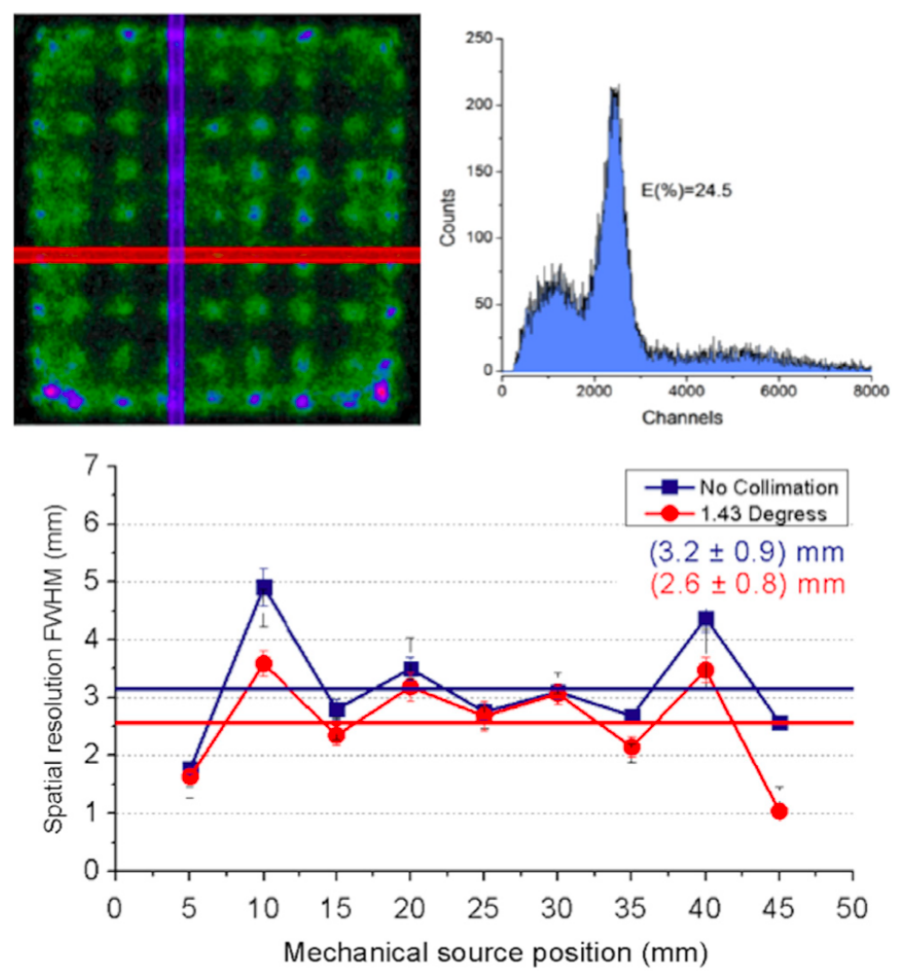

Fig. 6. Top-left: flood map for the $11 \times 11^{22} \mathrm{Na}$ sources. Top-right graph: energy profile for a small region. Bottom: mean spatial resolution measured for the horizontal sources marked on the flood plot, before and after applying electronic collimation of 1.43 .

\section{Conclusions}

An ASIC readout was widely evaluated during this study for its potential use in combination with monolithic scintillator crystals. Experimental results with pixelated crystals proved the ASIC capability to provide good results in term of energy, spatial and timing resolution. Moreover, it should be noticed the capability of the detector block to resolve $32 \times 32$ pixels, $1.6 \mathrm{~mm}$ size, using 
$6.33 \mathrm{~mm}$ pitch SiPMs. We did not observe significant temperature or SiPM bias dependence on the crystal pixel identification. We would also like to pinpoint the capability to reach $330 \mathrm{ps}$ FWHM with the described ASIC version but using $6 \times 6 \mathrm{~mm}^{2}$ SiPM.

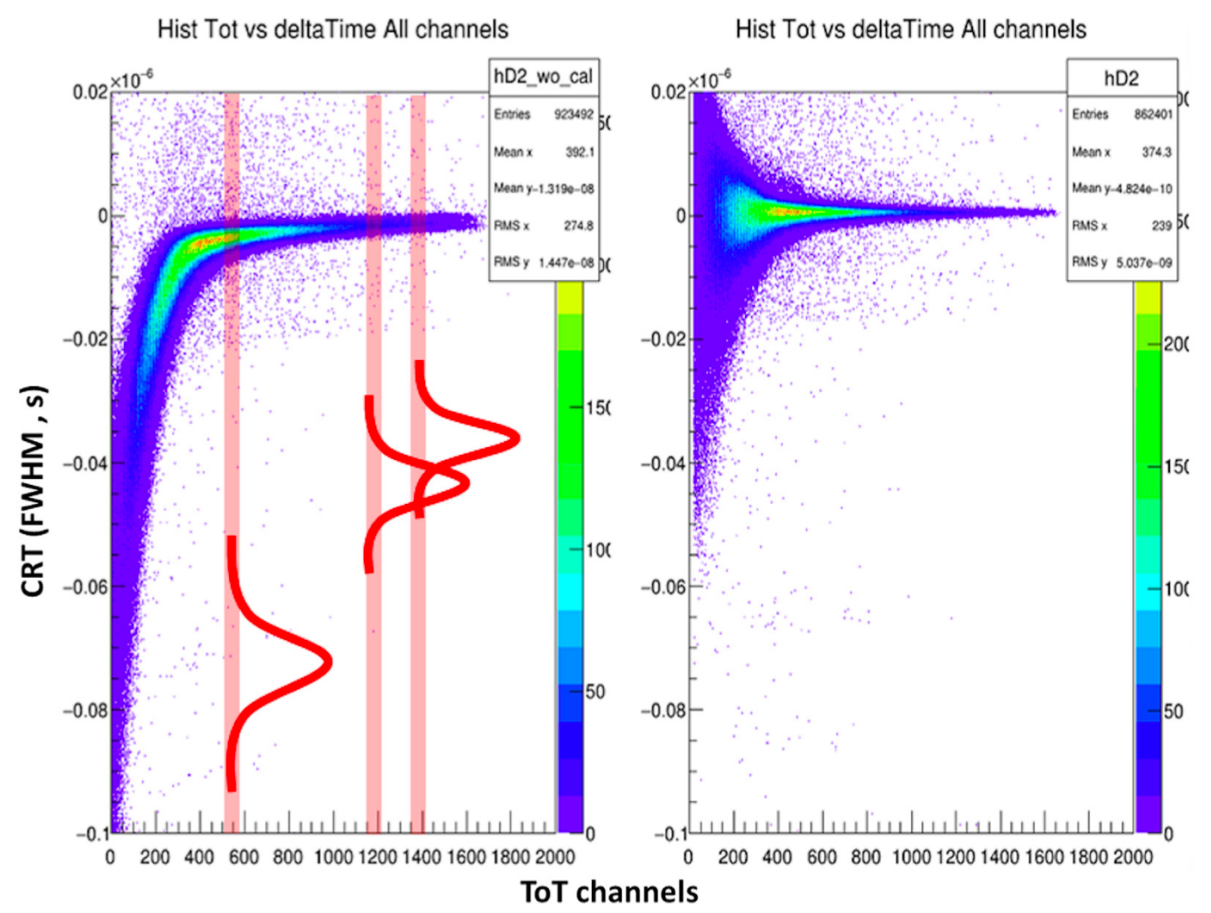

Fig. 7. Top: 2D histograms of the CRT measured as a function of the ToT collected for all channels. The left one corresponds to the one before time alignment. The right one is the result after compensating the events by the measured offset.

Concerning the measurements with monolithic blocks, promising results were obtained despite the challenges shown up when analog SiPMs and monolithic blocks are combined. Further investigations will be carried out using thicker 15-20 mm crystals, as used for clinical systems. The obtained timing result is expected to be improved after applying averaging methods to the timestamp determination instead of considering only the SiPM channel with the highest amount of light. During those measurements with monolithic blocks, we observed an average of 10 SiPM pixel fired per impact. Early results have been shown that after applying averaging timestamp methods (energy, distance to impact, time walk compensation, etc.) it is feasible to achieve accurate timing resolution [6].

Future work includes the upgrade of this current ASIC chip with the TOFPET2 ASIC that is capable of providing charge integration of signal pulses to overcome ToT limitations. This upgrade is expected to significantly improve the results, especially in terms of energy resolution. Furthermore, in the section of timing resolution, huge improvement is expected after performing threshold scans as well as after applying the previous referred averaging methods for timestamps belonging to same impact. 


\section{Acknowledgments}

This project has received funding from the European Research Council (ERC) under the European Union's Horizon 2020 research and innovation program (grant agreement No 695536). It has also been supported by the Spanish Ministerio de Economía, Industria y Competitividad under Grants No. FIS2014-62341-EXP and TEC2016- 79884-C2-1-R.

\section{References}

[1] S. Surti and J.S. Karp, Design considerations for a limited-angle, dedicated breast, TOF PET scanner, Phys. Med. Biol. 53:11 (2008), 2911-2921.

[2] S. Surti and J.S. Karp, Advances in time-of-flight PET, Phys. Med. 32:1 (2016), 12-22.

[3] A. Aguilar, et al., Pilot tests of a PET detector using the TOF-PET ASIC based on monolithic crystals and SiPMs, Journal of Instrumentation. 11 (2016), C12033.

[4] M.D. Rolo, et al., TOFPET ASIC for PET applications, Journal of Instrumentation 8 (2013), C02050.

[5] K.E. Hoff, et al., Fast computation of generalized Voronoi diagrams using graphics hardware, in: Proceedings of the 26th Annual Conference on Computer Graphics and Interactive Techniques, 1999, 277-286.

[6] H. T. van Dam et al., Sub-200 ps crt in monolithic scintillator pet detectors using digital sipm arrays and maximum likelihood interaction time estimation, Phys. Med.Biol. 58:10 (2013), 3243-3257. 


\subsection{TOF-PET Detectors Based on ASIC Technology and Analog SiPMs}

Following the prior contribution which described our pilot tests with monolithic and pixelated crystals, through the present one, we intend to provide here a more complete overview of the capabilities of the detector blocks in terms of spatial resolution. Moreover, we also introduce, for the first time in this $\mathrm{PhD}$ thesis, the concept of averaging timestamps and, thus, the resulting single timing information when multiple photosensor contribute of a single gamma-ray event.

For these experiments, an improved set-up was designed and built. The readout was upgraded to the TOFPET2 ASIC while new custom printed circuit boards were also designed. Detailed tests were first carried out again with a crystal array of $32 \times 32$ elements of $1.5 \times 1.5 \mathrm{~mm}^{2}$ each. As the reader will observe, all crystal elements were very well resolved. Notice that this matrix was coupled to a SiPM array of $8 \times 8$ photosensors with $6 \times 6 \mathrm{~mm}^{2}$ each, without any light guide in between scintillator and photosensor. Thick monolithic blocks were thereafter used in the experiments. They have dimensions of $50 \times 50 \times 15 \mathrm{~mm}^{3}$ treated with black paint in all lateral walls. Additionally, the entrance face included a novel retroreflector layer widely use in our research group, which bounces back the optical photons to the emission point and, therefore, improves the light collection at the time the light distribution is preserved. In order to determine the spatial resolution, an array of $11 \times 11^{22} \mathrm{Na}$ sources with $1 \mathrm{~mm}$ diameter and $4.6 \mathrm{~mm}$ pitch, was attached to one detector while both mechanical and software collimation was applied. Eventually, as it will be demonstrated in the framework of the study, a spatial resolution near 1 $\mathrm{mm}$ was reached for the sources at the center of the detector block while this slightly degraded towards the edges.

This contribution aims to shed light on two major aspects of the timing determination when using monolithic crystals, or configurations inferring light sharing among several photosensors. In particular, this contribution cites the fact that individual channels in the electronic chain suffer from skew time differences. These differences can be significantly large within a single chip (64 channels) and, thus, they should be compensated. In addition to this finding, we also began with the concept of timestamp averaging, as it is observed when using the monolithic crystal in this contribution, by increasing the number of photosensors information, an improvement of the global timing resolution is achieved. 


\title{
TOF-PET Detectors Based on ASIC Technology and Analog SiPMs
}

Efthymios Lamprou, Andrea González-Montoro, Gabriel Cañizares, Victor Ilisie, Filomeno Sánchez, Marta Freire, Liczandro Hernández, Luis F. Vidal, John Barrio, Antonio J. González and José M. Benlloch

Published: IEEE Nuclear Science Symposium and Medical Imaging Conference Proceedings (NSS/MIC), Sydney, Australia, 2018, Pages 1-4.

DOI: https://doi.org/10.1109/NSSMIC.2018.8824517

\begin{abstract}
In this manuscript, detector blocks for Positron- Emission-Tomography (PET) are being evaluated for their integration in a TOF-PET system. In this contribution, detector concepts based on analog SiPM photosensors have been tested along with different types of scintillation materials. Firstly, pixelated crystals have been evaluated showing accurate results in terms of spatial, energy and timing resolution. Then, monolithic blocks were coupled to large SiPM photosensors arrays to determine their capabilities to resolve the gamma impacts in terms of position and timing. The read-out and the digitation of all signals were performed by a commercially available Application-Specific-IntegratedCircuit (ASIC) named TOFPET2. The obtained results as well as some methodologies that improve the timing performance of the monolithic blocks, are being described in this paper.
\end{abstract}

\section{Introduction}

Recent advances in front-end electronics have made feasible the development of highperformance gamma ray detectors. State-of-the-art PET detectors, capable to reach sub-200 ps Coincidence Timing Resolution (CTR) have been already presented and in depth evaluated [1].

Typically, these detectors are developed using pixelated crystals as the scintillation material. As this configuration permits the collection of a very high number of photons in a very short time and in a given photosensor element, it has been shown to be the optimal solution for achieving an accurate timing resolution. However, this detector approach may infer some limitations in terms of accurate decoding of the gamma event impact position especially regarding Depth- OfInteraction information (DOI) and, thus, alternatives are being explored [2].

Our aim is to evaluate PET detectors based on monolithic blocks and analog SiPM arrays. This detector configuration, as it has been already demonstrated, can provide accurate results to decode each annihilation photon. The wide spread of the produced scintillation light results in a very poor Signal-To-Noise ratio (SNR) that affects the timing resolution. Apparently, the poor SNR may lead to false triggering and noise eventually limiting the system timing performance [3]. 
In this manuscript we present experimental results based on both pixelated and monolithic crystals. The use of pixelated crystals facilitates the evaluation of the readout and may reveal the limits in terms of CTR. Analysis in terms of spatial, energy and timing resolution is carried out to explore the capabilities of both approaches. We have put emphasis on the timing capability tests with the monolithic blocks. In this work, crystal arrays of various pixel sizes are evaluated when coupled to the same array of SiPM. This work aims to shed light on different detector configurations, suitable for TOF-PET systems. The current analysis provides a better understanding of the light sharing effect when using crystal arrays, the limitations that show up but also possible ways to compensate them.

\section{Materials and Methods}

\subsection{ASIC based readout}

In order to process each SiPM photosensor element independently, we make use of a multichannel ASIC-based readout. In detail, we have used the TOFPET2 ASIC (see Fig. 1, topleft), a high performance 64-channels chip that integrates a quad-buffered TDC (30 ps time binning) and linear charge integrators for each channel. Its low configurable threshold for timing and its high event rates capabilities, make it suitable for light sharing applications [4]. Light sharing applications stand for scintillation light collection produced by a single gamma ray that fires multiple photosensors.

\subsection{Detectors Set-Up}

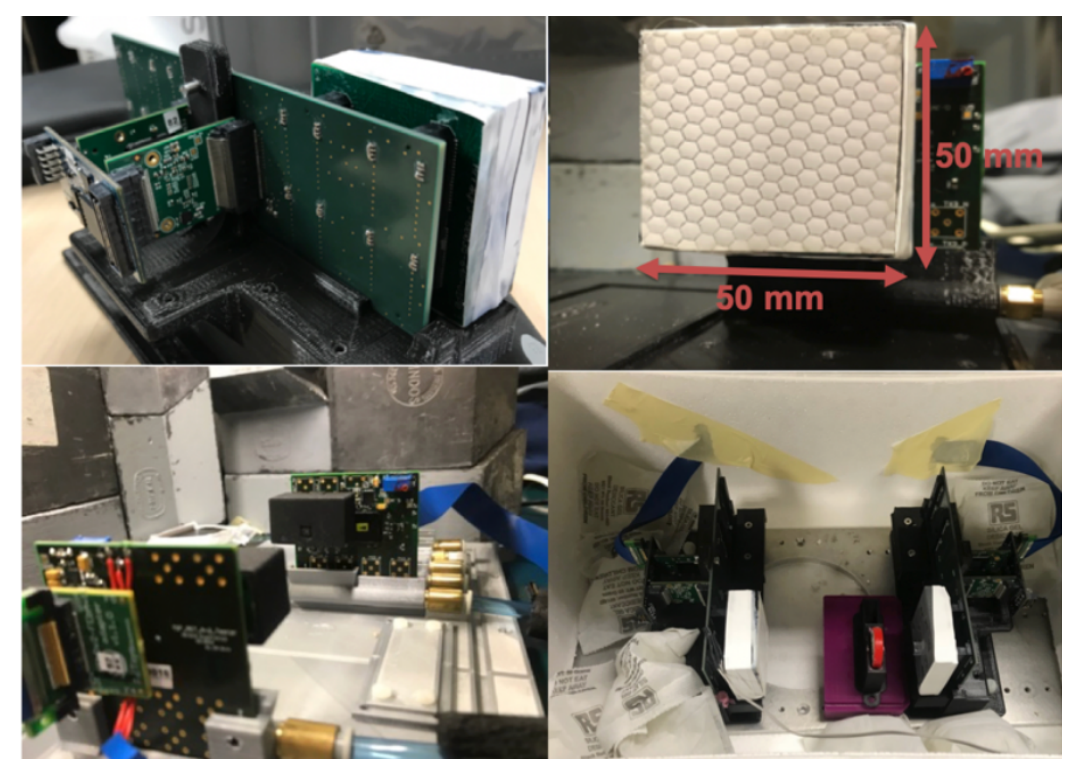

Fig. 1. Top: Left. TOFPET2 Front-End-Module. Right. Monolithic block, black painted with the entrance layer coupled to a retroreflector layer. Bottom: Left. Set-up based on the PM3325 SiPM arrays and single LYSO crystals. Right. Experimental set-up based on a monolithic block and a single LYSO pixel for the time skew correction and CTR evaluation. 
Aiming to characterize the ASIC performance and reveal the limits in terms of CTR, two small crystals $3 \mathrm{~mm} \times 3 \mathrm{~mm} \times 5 \mathrm{~mm}$ were coupled to the KETEK PM3325-WB SiPMs and coincidences measurements were carried out (Fig. 1, bottom-left).

In addition to the one-to-one coupling experiments, a pair of large $8 \times 8 \mathrm{SiPM}$ arrays with $6 \times 6 \mathrm{~mm}^{2}$ active area (J-Series, SensL), were coupled to crystal arrays with $1.6 \mathrm{~mm}$ pixel size and $6 \mathrm{~mm}$ height. Finally, the aforementioned SiPM arrays were used to characterize a monolithic block of $50 \mathrm{~mm} \times 50 \mathrm{~mm} \times 15 \mathrm{~mm}$ with black painted lateral walls and a retro-reflector $(\mathrm{RR})$ layer at the entrance, in terms of CTR and position (Fig. 1, top-right). When testing the impact position accuracy in the monolithic crystal, an identical reference detector was used facilitating the evaluation (see Fig. 1, bottom-right). Finally, a stable temperature environment was settled for all experiments, to avoid gain drifts (temperature variation below $2^{\circ} \mathrm{C}$ ) that may influence the evaluation results

\section{Results}

\subsection{Pixelated crystals}

CTR analysis of the tests carried out using the small LYSO crystals, verified ASIC's capabilities. In a measurement with 11000 valid coincidences events, a CTR nearing 200 ps FWHM was determined together with a good energy resolution of about $12 \%$ for both detectors (see Fig. 2) at a temperature of $18^{\circ} \mathrm{C}$.
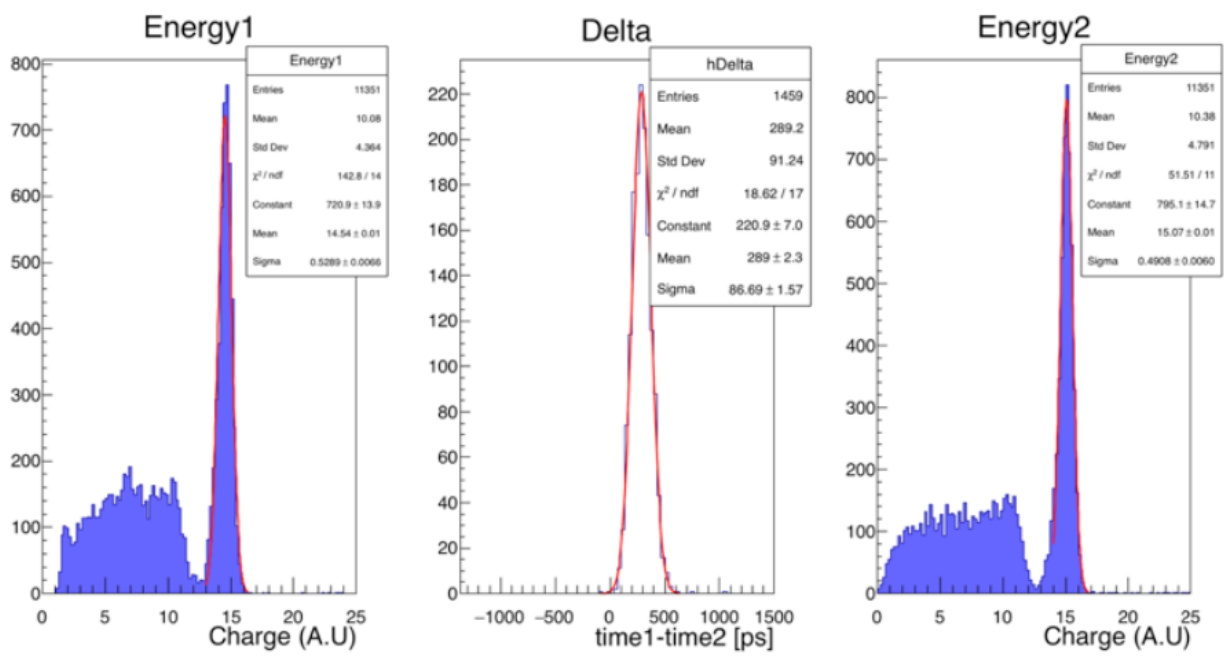

Fig. 2. Time resolution determined at 202 ps FWHM using PM3325-WB SiPMs and one-to-one-coupling. Temperature was stabilized at $18^{\circ} \mathrm{C}$.

Using the LYSO crystal arrays and the large SiPM photosensors arrays, an estimation of the spatial resolution was obtained. As it can be observed in Figure 3, we have been able to resolve very well all $32 \times 32$ crystal elements with a very good SNR and an energy resolution better than $16 \%$. 

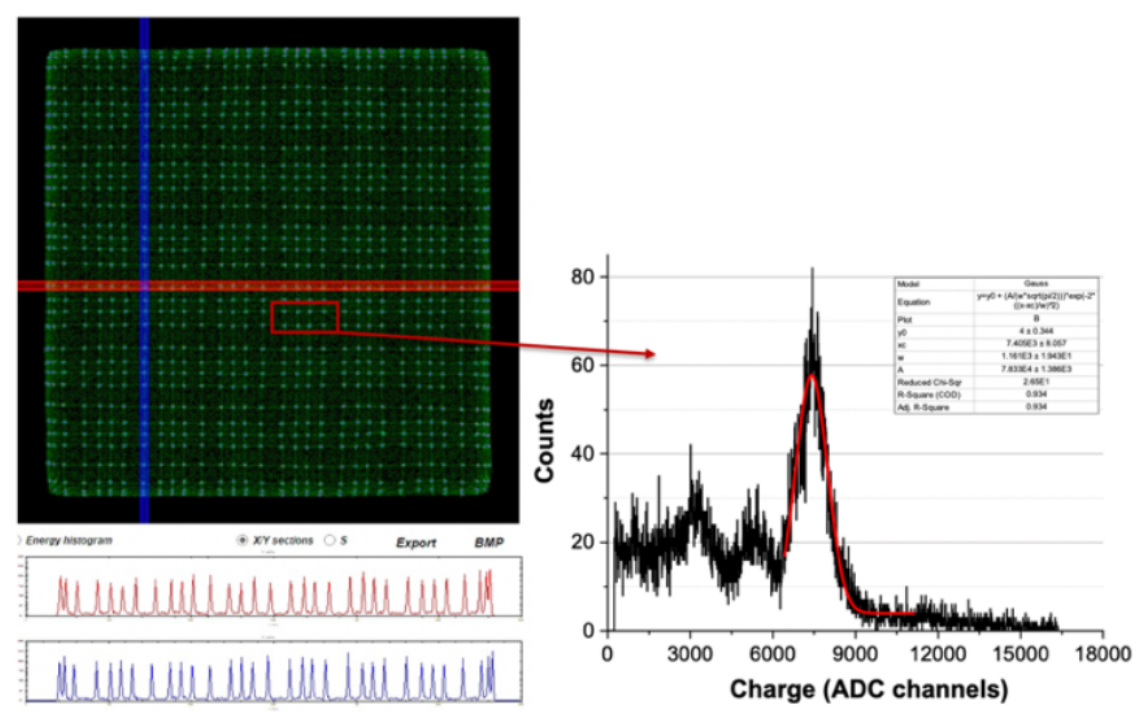

Fig. 3. Flood map showing all $32 \times 32$ crystal elements of $1.6 \mathrm{~mm}$ size, along with a good SNR and energy resolution near $16 \%$ FWHM.

\subsection{Monolithic blocks}

The monolithic based detectors were evaluated in terms of spatial and energy resolution. An array of $11 \times 11^{22} \mathrm{Na}$ sources with $1 \mathrm{~mm}$ diameter and $4.6 \mathrm{~mm}$ pitch, was attached to the one detector. Both mechanical and software collimation we have applied, allowing us to study the spatial resolution of these detector blocks. As it can be seen in Figure 4, a spatial resolution near $1 \mathrm{~mm}$ is obtained for the sources in the center of the block while it slightly degrades towards the edges.
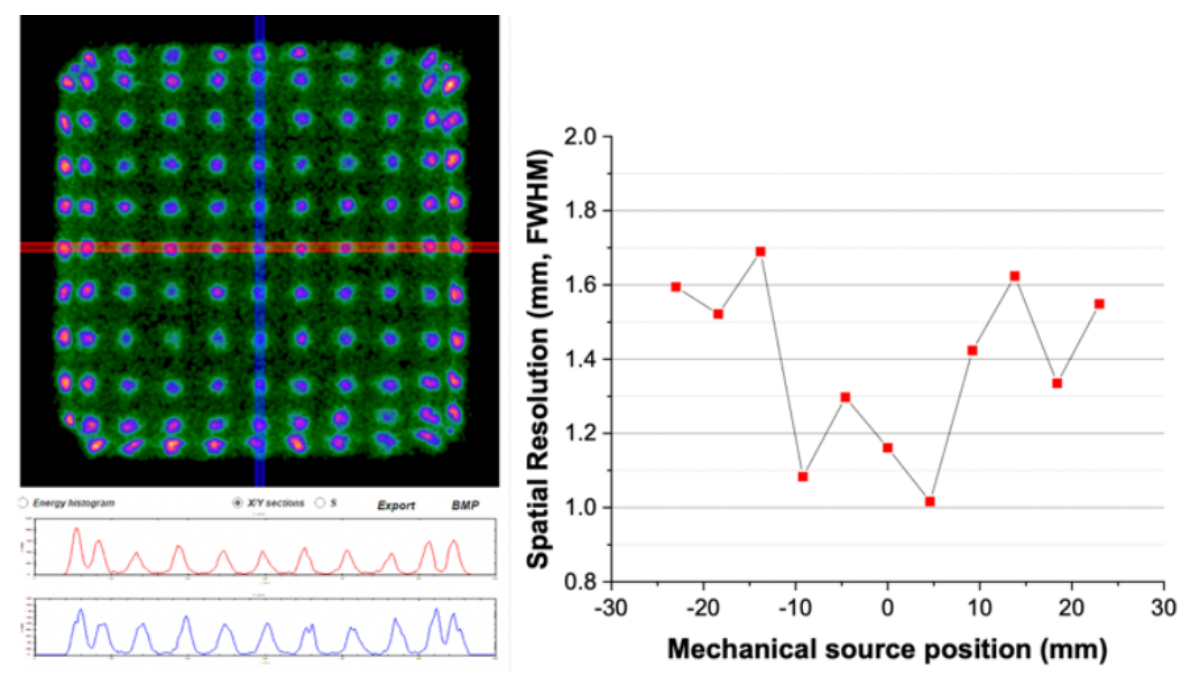

Fig. 4. Left. Flood map of the measured $11 \times 11$ collimated ${ }^{22} \mathrm{Na}$ sources, (pitch between sources is $4.6 \mathrm{~mm}$.) Right. Spatial resolution measured for a central raw of sources calibrated to $\mathrm{mm}$.

As mentioned above, the wide scintillation light distribution results in a poor SNR. As more as 30 SiPMs are typically fired per gamma impact when using a low threshold. Setting a low 
threshold in the applications with light sharing is critical, as the gain seen in this kind of applications is dramatically low compared to the one seen when using the one-to-one coupling approach. Thus, in the case of combining analog SiPM photosensors and monolithic crystals, intense degradation in time resolution occurs, as the system becomes sensitive to false triggering by the dark counts and to time walk error.

To initially characterize the CTR in the continuous crystal, we carried out coincidence measurements with a reference detector composed by a single LYSO pixel $6 \times 6 \times 15 \mathrm{~mm}^{3}$. Aiming to use a low energy threshold, critical found be the reduction of the dark count rate to avoid false triggering. For that purpose, we performed the measurements while keeping a stable temperature environment near $7^{\circ} \mathrm{C}$. As expected, the analysis of the raw data revealed significantly worst CTR compared with the one obtained when using smaller crystals or one-toone coupling. Time resolution over $1 \mathrm{~ns}$ FWHM was initially measured showing the necessity for calibration methods. It is considered that the poor timing performance of the blocks is provoked by three main factors. Firstly, the time skew among ASIC channels. The variations in time-paths across the channels can be up to $1 \mathrm{~ns}$ (see Fig. 5), increasing significantly the uncertainty of the Coincidence Resolving Times (CRT), deteriorating in that way the timing resolution. In addition, as explained above, the poor SNR introduces the time walk uncertainty of the signals. Finally, the light speed propagation inside the $15 \mathrm{~mm}$ of thickness monolithic crystal also deteriorates the CRT.

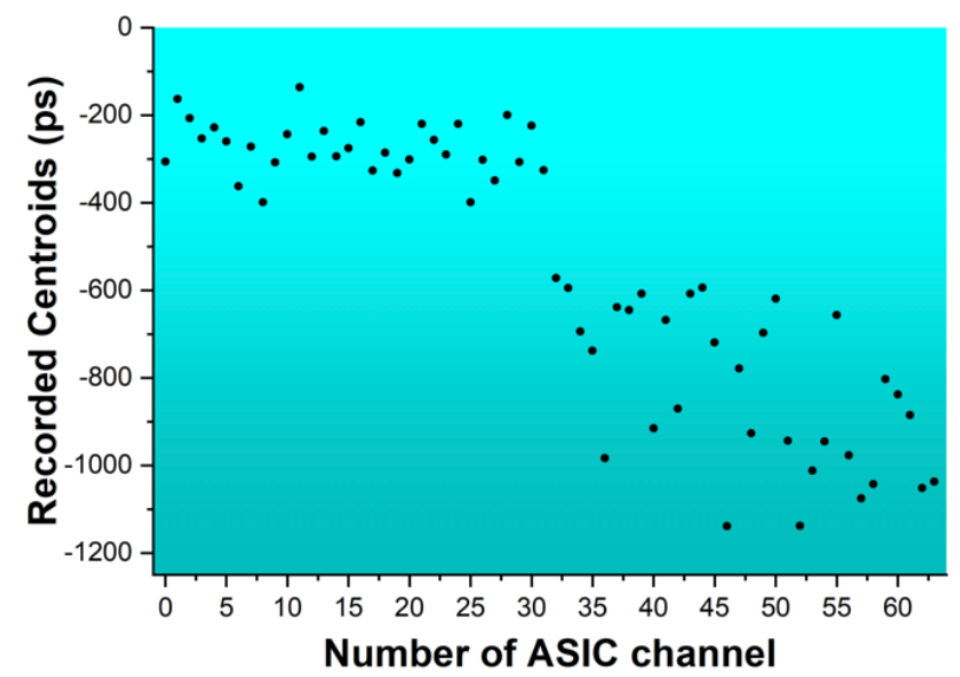

Fig. 5. Dispersion of the RAW centroids of the 64 ASIC channels.

In order to deal with the time skew, the method proposed and described in the current manuscript is the following. We plotted the time differences between each channel of the monolithic block and the reference detector after applying an energy filter to both detectors photopeaks. In addition, for this particular calibration method, events that occurred only near the photosensor (lower DOIs) were chosen so to minimize as much as possible the timing uncertainty. 
So, in total 64 histograms were obtained. We used Gaussian fits to obtain the centroids as well as the sigma for each pair of channels. Considering that the reference detector contribution was stable for all virtual coincidence pairs, we could use the recorded centroids as a timestamp offset for each channel of the monolithic based detector block.

An algorithm for online processing was developed for correcting all timestamps of all following measurements using the information previously obtained. The result of this method, is that the timing distribution when considering all channels is centered to near zero values and the timing resolution is improved as expected for about 200 ps FWHM (Fig. 6).
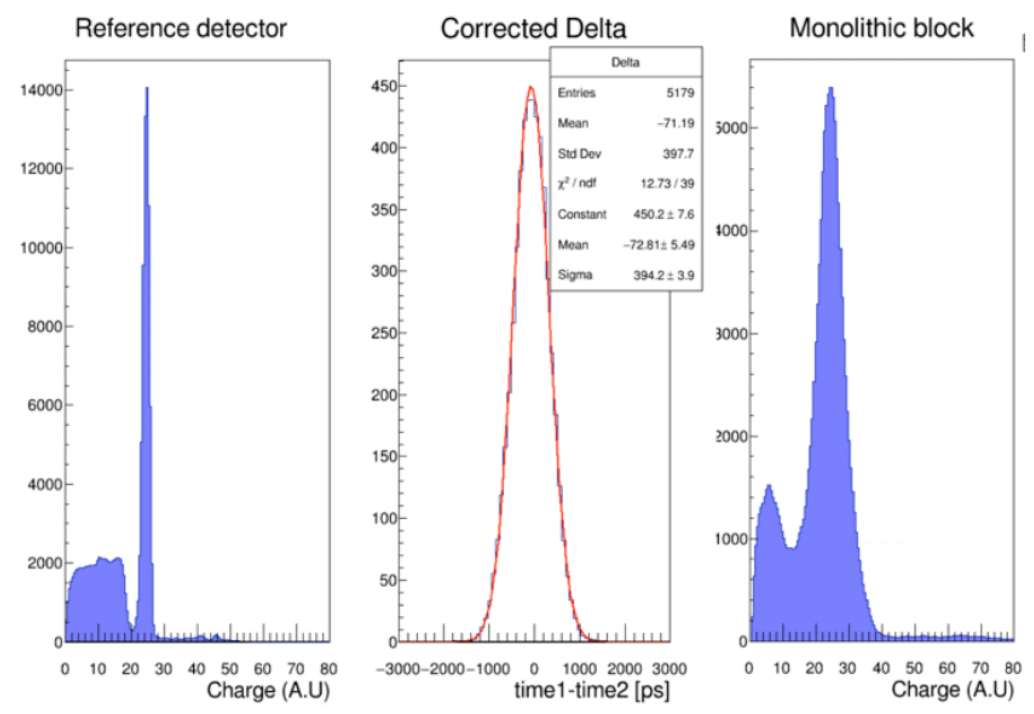

Fig. 6. After applying the time-skew correction in the following measurements, the timing distribution is centered close to zero while the timing resolution is also improved.

Further improvement of the CTR was achieved when an approach for averaging timestamps was used. Instead of the typical assignment of the event timestamp to the first ASIC channel that crossed the threshold, we made use of a timestamp averaging method where each timestamp was weighed by its energy [5]. The advantages of this method are mainly two. First of all, it can lead to partial compensation of the time walk error and overcome some false triggering. In addition, as several timestamps are considered it may improve the dependency from the light speed propagation in the crystal. As it can be seen in Figure 7, averaging of up to the nine earliest recorded timestamps was tested. Significant improvement of the CTR was seen for all approaches but the best results were given when using the earliest seven timestamps.

Pilot results shown that after weighting the timestamps, considerable improvement is achieved, in some cases of over 100 ps FWHM reduction. It should be noted, that the timing results obtained with the monolithic block and presented in this paper have not been processed any further. In the light sharing applications, a calibration method of the time-walk dependency of the signals, becomes critical. Methods have been suggested from our group that in some cases 
lead to a significant improvement [5]. In particular, the time skew correction in combination with a simple energy weighting averaging method of timestamps can significantly improve the results as shown above. Those corrections can improve from a RAW timing resolution of about $1.1 \mathrm{~ns}$ CRT FWHM to a one as good as 770 ps. Moreover, when the time walk correction would be optimized, we expect CRT values near 500 ps FWHM for this particular detector configuration.

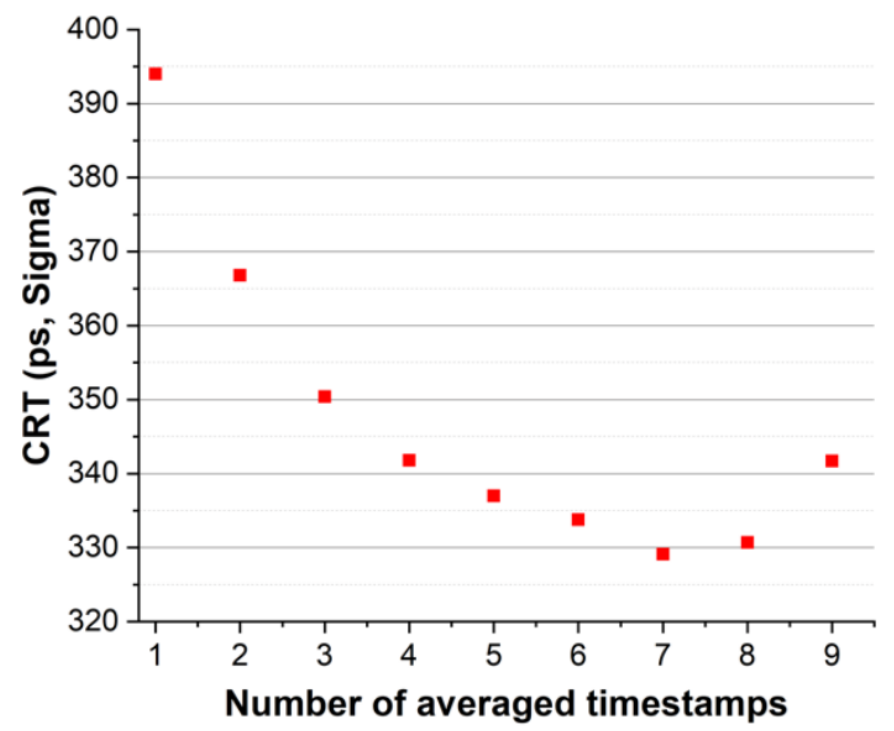

Fig. 7. Diagram shows the time resolution as a function of the number of the averaged-energy weighed timestamps.

\section{Conclusions}

Nowadays, the use of an ASIC for processing the signals coming from analog photosensors seems to be a very efficient approach. As it was described in the present manuscript, using the TOFPET2 ASIC, accurate results in terms of position and timing resolution were obtained. Spatial resolution well below $1.5 \mathrm{~mm}$ was obtained and when using the one-to-one coupling method, timing resolution as good as 202 ps FWHM was achieved, revealing in that way the ASIC capabilities.

Some depth evaluation of the monolithic based detector was also carried out. Monolithic crystals due to the accurate position decoding of gamma ray impacts over alternative pixelated crystals, become an interesting line of research. Results shown before, come to an agreement with this statement. Spatial resolution near $1 \mathrm{~mm}$ was achieved which in addition to the DOI information can lead to a significant improvement of the accuracy of reconstructed images. Of course, challenges are showed up when aiming to extrapolate accurate timing information from this type of scintillator material when combined with analog photosensors, but as we are aiming to prove, several methods can be designed to improve the performance and reach to TOF-PET detector with real 4D capabilities. 


\section{Acknowledgments}

This project has received funding from the European Research Council (ERC) under the European Union's Horizon 2020 research and innovation program (grant agreement No 695536). It has also been supported by the Spanish Ministerio de Economía, Industria y Competitividad under Grant TEC2016-79884-C2-1-R.

\section{References}

[1] P. Lecoq, Development of new scintillators for medical applications. Nucl. Instrum. Methods Phys. Res. A. 809 (2016), 130-139.

[2] A. J. González et al., A PET Design Based on SiPM and Monolithic LYSO Crystals: Performance Evaluation, IEEE Trans. Nucl. Sci. 63 (2016), 2471.

[3] E. Lamprou et al., PET detector block with accurate 4D capabilities. Nucl. Instrum. Methods Phys. Res. A. 912 (2018), 132-136.

[4] A. Di Francesco et al., TOFPET2: A high-performance ASIC for time and amplitude measurements of SiPM signals in time-of-flight applications. Journal of Instrumentation. 11:03 (2016), C03042.

[5] E. Lamprou, et al., Characterization of TOF-PET Detectors Based on Monolithic Blocks and ASIC-Readout. arXiv: Instrumentation and Detectors, 2018. 


\subsection{Exploring TOF capabilities of PET detector blocks based on large monolithic crystals and analog SiPMs}

In the present contribution we give emphasis to the TOF capabilities of the monolithic scintillators. Shortly, besides describing the efforts devoted on improving the precision in the time determination of each gamma-ray event, we aim to shed light on the behavior of the scintillation light sharing inside these continuous crystals, and study its impact in the timing performance.

We started evaluating the detector set-ups based on single crystal elements and the revised ASIC version. The aim of these experiments was to reveal the limits of the electronics in terms of time resolution. Afterwards, a monolithic based detector, identical to the one described in the prior work $\left(50 \times 50 \times 15 \mathrm{~mm}^{3}\right)$ was assembled to be tested in coincidence mode. Herein, we first show the deep analysis carried out for factors related with the scintillation light distribution. Regarding the timing evaluation with large monolithic blocks, we still found several challenges resulting in a poor RAW coincidence time resolution. A series of calibration and post-processing procedures were followed, which among others included time-skew and time-walk calibration, timestamp averaging and noisy events discarding. Eventually, following these methods, we were able to achieve the value of 497 ps FWHM for impacts along the whole monolithic crystal, which improved to 371 ps FWHM for specific volumes of interest.

This achievement with monolithic is still far from the desired values. However, we should keep in mind that the size of the tested monolithic crystal and SiPM arrays, were chosen with the aim to not compromise others critical characteristics like spatial resolution, sensitivity and cost. For instance, based on our studies, different crystal treatments, photosensor types and of course, crystal dimensions, can directly boost timing performance and show values well below $300 \mathrm{ps}$ FWHM.

The whole concept of this contribution aimed to demonstrate in detail the challenges arisen in the timing determination when using thick monolithic blocks. We consider that the methods proposed here can be effectively applied in all light sharing configurations, permitting a significant improvement in terms of performance. According to our knowledge, this is the very first work in which monolithic blocks are tested using analog SiPMs and an ASIC readout, for their potential capabilities in TOF-PET imaging. 


\title{
Exploring TOF Capabilities of PET Detector Blocks Based on Large Monolithic Crystals and Analog SiPMs
}

\author{
Efthymios Lamprou, Antonio J. Gonzalez, Filomeno Sanchez, and Jose M. Benlloch \\ Published: Physica Medica, Volume 70, Pages 10-18, 2020. \\ DOI: https://doi.org/10.1016/j.ejmp.2019.12.004
}

\begin{abstract}
Monolithic scintillators are more frequently used in PET instrumentation due to theirs advantages in terms of accurate position estimation of the impinging gamma rays both planar and depth of interaction, their increased efficiency, and expected timing capabilities. Such timing performance has been studied when those blocks are coupled to digital photosensors showing an excellent timing resolution.

In this work we study the timing behaviour of detectors composed by monolithic crystals and analog SiPMs read out by an ASIC. The scintillation light spreads across the crystal towards the photosensors, resulting in a high number of SiPMs and ASIC channels fired. This has been studied in relation with the Coincidence Timing Resolution (CTR). We have used LYSO monolithic blocks with dimensions of $50 \times 50 \times 15 \mathrm{~mm}^{3}$ coupled to SiPM arrays $\left(8 \times 8\right.$ elements with $6 \times 6 \mathrm{~mm}^{2}$ area) which compose detectors suitable for clinical applications.

While a CTR as good as $186 \mathrm{ps}$ FWHM was achieved for a pair of $3 \times 3 \times 5 \mathrm{~mm}^{3}$ LYSO crystals, when using the monolithic block and the SiPM arrays, a raw CTR over 1 ns was observed. An optimal timestamp assignment was studied as well as compensation methods for the time-skew and time-walk errors. This work describes all steps followed to improve the CTR. Eventually, an average detector time resolution of 497 ps FWHM was measured for the whole thick monolithic block. This improves to 380 ps FWHM for a central volume of interest near the photosensors. The timing dependency with the photon depth of interaction and planar position are also included.
\end{abstract}

\section{Introduction}

Since the development of the firsts Positron Emission Tomography (PET) scanners already back in 80's, several efforts have been devoted to provide an accurate timing determination of the $511 \mathrm{keV}$ annihilation photons [1][2][3]. This information, typically known as Time-Of-Flight (TOF), is directly impacting improvements in the contrast of the reconstructed medical images [4]. Unarguably, the continuous research in this field and the potential achievement in the socalled Coincidence Time Resolution (CTR) to values below 100 ps Full-Width-at-Half-Maximum (FWHM), will lead to a certain technological revolution of both clinical and pre-clinical PET practice [3].

In order to achieve an excellent time resolution in PET instrumentation, several factors need to be considered, such as an efficient photosensor exhibiting a fast rise time, high quantum efficiency (QE) and relatively high gain [6]. A photosensor with these characteristics and very often used in gamma ray detectors is the photomultiplier tube (PMT). They have been used in several applications, demonstrating also its feasibility to be essential components in TOF-PET 
detectors [6][7]. An alternative photosensor device is the silicon photomultiplier (SiPM) [8][9]. Latest works show that SiPM are gaining ground over the PMTs in gamma-ray detectors due to their compact size, their compatibility to magnetic fields, and a high photodetection efficiency (PDE). Shortly, the operating principle of SiPMs is based on the sum of all internal single-photon avalanche diodes (SPADs). This inherently introduces some uncertainty in the event timestamp generation. An alternative approach to SiPM was introduced by Philips Digital Photon Counting (Aachen, Germany), with the so-called digital silicon photomultipliers (dSiPMs). In their architecture, each cell is composed by its independent SPAD and its readout electronics, and it is capable of detecting exactly one photon. Detailed descriptions of their working principle and characteristics can be found [9][11].

Another key element in the performance of a detector block is the scintillation crystal. Recent advances in this area have well enabled the development of TOF-PET systems [12][13][14]. A scintillator crystal suitable for TOF-PET detectors, besides high stopping power, must also exhibit high initial photon intensity [4]. This characteristic can be achieved by an adequate light output and a short decay time.

Nowadays several crystal types and compositions suitable for TOF are available [15]. There are mainly two types of scintillators used in gamma ray detectors namely pixelated crystals and monolithic blocks. Both types are briefly described below, while emphasis in the present work is given to the second type.
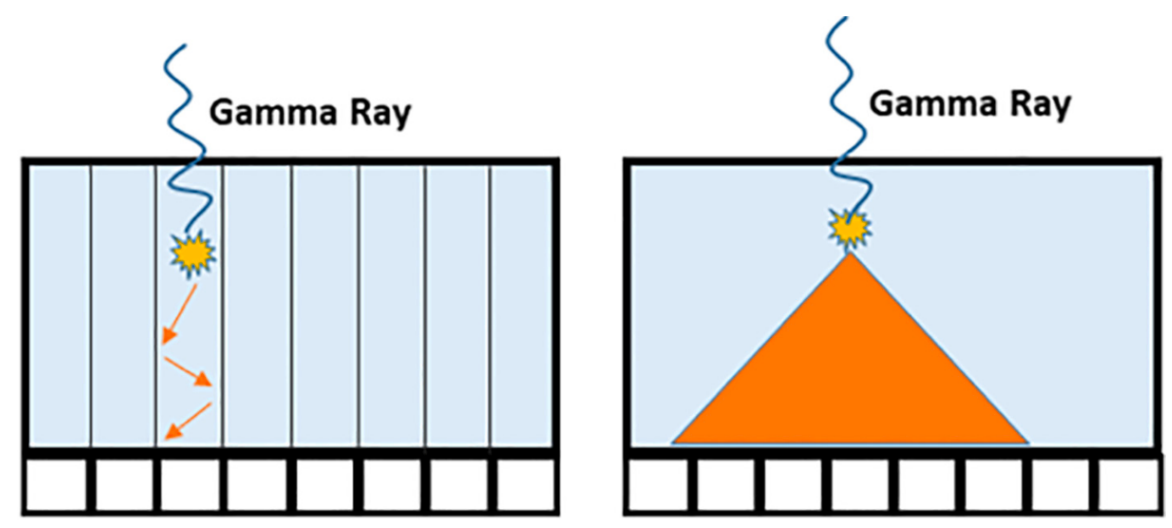

Fig. 1. Representation of the scintillation light distribution for one gamma event inside a pixelated crystal (left) and a monolithic block (right).

When aiming to achieve a very good timing resolution, the claimed most efficient approach is to use arrays of pixelated crystals with pixel dimensions that match that of the photosensor element active area, dubbed as one-to-one coupling. An example of this approach can be seen in Fig. 1 left. In this configuration, after some internal reflections of the generated optical photons inside the crystal pixel, they eventually exit and are collected mainly by a single photosensor element with no significant losses to neighbor photosensors. This allows one to collect high amounts of visible photons at a given short time frame. The main degradation observed in this 
case is some delay of the optical photons to reach the photosensors due to the light transfer efficiency (LTE) and the light transfer time spread (LTTS) [6][16]. Moreover, the detector spatial resolution is limited to the pixel size. The deterioration in the CTR increases for longer light paths meaning for thicker scintillators. However, this difference does not exceed some tenths of picoseconds. An alternative detector configuration towards improving the detector block spatial resolution makes use of crystal arrays with pixel sizes smaller than the photosensor elements, implying scintillation light sharing among few photosensors [17]. Optical lightguides are employed avoiding accumulation of events in a single photosensor. This approach tends to degrade the CTR due to the spread of the optical photons among neighbor photosensor elements.

Detector block configurations which make use of monolithic crystals provide some advantages when compared to pixelated crystals and, therefore, make them good candidates for PET applications [18][19][20][21]. The crystal thickness and geometry, as well as treatments on the walls vary depending on the application.

In monolithic blocks the scintillation photons are isotropically emitted travelling straight in all directions, differently from the pixelated crystal case in which the optical photons are trapped inside bouncing on the walls until they reach one photosensor. The light spread in the monolithic block permits an accurate position decoding of the gamma ray impact, being a convenient choice for a high intrinsic detector spatial resolution [6]. In addition to the position, a monolithic scintillator could ideally show a better timing performance compared to a pixelated one, due to the fact that the generated optical photons are not suffering from the aforementioned internal reflections inside the crystal pixel introducing time delays. However, the wide spread of the scintillation light does not facilitate the collection of a high number of photons at each single photosensor element in a very short time, which is mandatory for a good TOF. The poor collection of optical photons and the resulting low Signal-To-Noise ratio (SNR) for each channel, leads to noise and false signal triggering. In order to reach a good CTR, it is critical to use high performance readout electronics especially sensitive to the first photoelectrons. Ideal candidates for this purpose are the aforementioned dSiPMs, but also novel Application Specific Integrated Circuits (ASICs) specifically designed with low noise electronics.

Few works have been published showing that dSiPMs can successfully be combined with monolithic blocks to provide accurate TOF information even below 200 ps FWHM [11][17]. Due to their operating principle, these photosensors can be sensitive to the very first photoelectrons while keeping the noise level very low. Typically, this is achieved by operating them at low temperatures of $-20^{\circ} \mathrm{C}$, while at the same time they show the capability to disable microcells with higher levels of noise.

In this work, we explore the limits, in terms of timing resolution, when large and thick continuous crystals are read by analog SiPMs and ASICs. Emphasis has been given in analyzing the contribution of all photosensors which are involved in each generated scintillation 
distribution, aiming to get a better understanding of the light shape and its relevance with respect to the timing information. Evaluation results as well as methods to improve the CTR are being presented and discussed, aiming to shed light on the limits of timing resolution for this kind of detector configurations.

\section{Materials and Methods}

\subsection{ASIC readout}

We have selected an ASIC to read, digitize and process all photosensors signals. All photosensors were individually read out avoiding reduction schemes introducing noise or additional delays in the time-paths of the signals. The ASIC used all throughout the measurements was the TOFPET2 (PETsys, Portugal). This particular chip can read up to 64 channels, and for each of them includes charge integration Analog-To-Digital Converters (ADCs), and Time-ToDigital Converters (TDCs) with 30 ps binning. Inside the ASIC, the incoming signal is evaluated by two analog circuit schemes, before it becomes a valid gamma signal. The first one is related to the timing of the signal and is composed by two discriminators. The first discriminator, namely vth_t1, uses a very low voltage threshold which typically corresponds to few photoelectrons and is designed to start the process. The output of this discriminator is fed into an AND gate after a programmable delay. To the same AND gate, the output of the second discriminator (vth_t2) is fed. vth_t2 is set to a higher voltage threshold in order to discard dark counts without introducing any dead time to the system. The output of the AND gate results in a trigger signal which generates the timestamp using a $200 \mathrm{MHz}$ clock. The second circuit scheme is based on a discriminator (vth_e) designed to discard pulses with relatively low amplitude and is operated as the energy threshold. Only when the three thresholds are met, a gamma-ray event is considered valid. Further information about the ASIC and the DAQ system can be found in references [24][25], among others.

\subsection{SiPM photosensors}

Two types of SiPM photosensors were used. A pair of SiPMs with $3 \times 3 \mathrm{~mm}^{2}$ active area (PA3325 model, KETEK, Germany) configured at a bias voltage of $31 \mathrm{~V}$ were tested with small pixel crystals. Other experiments were carried out using two $8 \times 8 \mathrm{SiPM}$ arrays with $6 \times 6 \mathrm{~mm}^{2}$ active area each (ON-Semi, J-series model). The wide total active area of these arrays suggested them as good candidates for their integration in clinical TOF-PET systems and especially in combination with large monolithic crystals [26]. Those arrays have an active coverage area of 92\% permitting the collection of high amounts of scintillation photons and, thus, improving the SNR. The larger capacitance of SiPMs with $6 \mathrm{~mm}$ will not significantly influence the CTR when combined with monolithic blocks, as the expected uncertainty due to the light spread might be larger [27]. Those SiPMs arrays were operated at two bias voltages, 29 and 30.5V, depending on the experiment. 


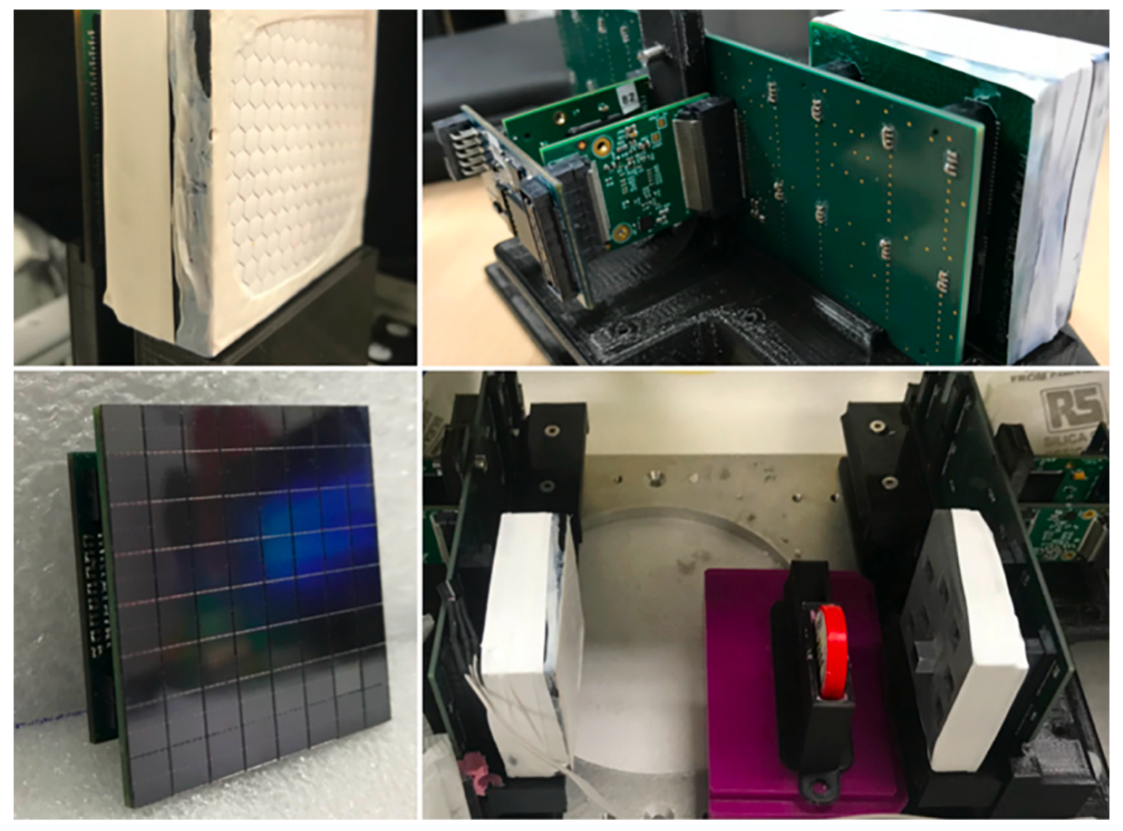

Fig. 2. Top-left, monolithic block $\left(50 \times 50 \times 15 \mathrm{~mm}^{3}\right)$ with a RR layer at the entrance. Top-right, TOFPET2 ASIC Front-End-Module. Bottom-left, SiPM $8 \times 8$ array with $6 \times 6 \mathrm{~mm}^{2}$ each photosensor element. Bottom-right, experimental set-up based on a monolithic block and a reference single pixel detector.

\subsection{Detector set-ups}

Two types of experiments were designed. First, pixelated crystals following the one-to-one coupling were tested. The aim was to characterize the ASIC and the whole DAQ system performance. A coincidence measurement was carried out using the PA3325 SiPM sensors coupled to LYSO crystals wrapped with Teflon of $3 \times 3 \times 5 \mathrm{~mm}^{3}$. Thereafter, experiments were carried out with two photosensor elements from the J-Series arrays and two LYSO crystal pixels covered with Enhanced Specular Reflector (ESR) of $6 \times 6 \times 15 \mathrm{~mm}^{3}$. For both experiments, the ASIC discriminators were set to their default values, that means vth_t $1=20$, vth_t $2=20$ and vth_e $=15$, respectively.

The monolithic LYSO crystals had dimensions of $50 \times 50 \times 15 \mathrm{~mm}^{3}$, matching the SiPM array dimensions. These crystals were treated with black paint in the four lateral walls in order to avoid undesired internal reflections which typically influence the spatial resolution. A retroreflector layer was added to the entrance face (Fig. 2 top-left). This particular optical element bounces back the light towards the emission point, improving the light collection at the photosensors while preserving the light distribution [20].

For the evaluation of the monolithic blocks, we first studied one detector block against a reference detector composed by an individual LYSO pixel of $6 \times 6 \times 15 \mathrm{~mm}^{3}$ coupled to one photosensor element of an identical SiPM array (Fig. 2 bottom-right). This approach provides an optimal characterization of the performance of the monolithic crystal, as it exhibits the minimum uncertainty in terms of timing. The two detectors were independently configured in terms of SiPM 
bias and thresholds. For the reference pixel-based detector, we used the same configuration as for the initial one-to-one coupling experiments ( $29 \mathrm{~V}$ and default thresholds). However, the detector with the monolithic block was set to $30.5 \mathrm{~V}$. Lower thresholds were used, since we observed a lower collection of photons per channel. In particular, the voltage discriminators vth_t1, vth_t2 and vth_e were set to 4, 8 and 8 DAQ units, respectively, meaning that the timestamp is generated at the first 1-3 photoelectrons. This set-up was also used during the calibration procedure designed to compensate the uncertainties in the timestamps introduced by the time-skew and time-walk errors, but also due to the SiPM energy non-linearity. The time-walk is referred to the dependency of the timing determination of a signal with its charge amplitude, while the time-skew refers to the timing error introduced by the different time-paths among ASIC channels, see section 3.4 for further details.

Afterwards, two identical detectors, both based on monolithic blocks were tested in coincidence. It should be noted that for the experiments using the SiPM photosensors array, custom printed circuit boards (PCBs) were developed, as an interface between the DAQ boards and the SiPM arrays (see Fig. 2 top-right).

All measurements were carried out at stable temperatures environment $\left( \pm 0.5^{\circ} \mathrm{C}\right)$ in the range of 7 to $19^{\circ} \mathrm{C}$, depending on the experiment. Small temperature drifts may affect the results and, thus, special attention to this aspect was taken. Moreover, the whole assembly was placed inside a light tight box. $\mathrm{A}^{22} \mathrm{Na}$ source $(1 \mathrm{~mm}$ in diameter, $475 \mathrm{kBq}$ ) was used for all experiments. All results mentioned below, have been obtained after applying about 30\% (350-650 keV) energy window around the $511 \mathrm{keV}$ photopeak.

\subsection{Analysis on the monolithic detector}

A simple Center-of-Gravity calculation was applied to estimate XY planar coordinates of each recorded gamma-ray event. Regarding the calculation of the $\mathrm{Z}$ coordinate, here referred as the Depth of Interaction (DOI), for each gamma-ray impact in the monolithic block we summed the energies collected for every row and column of the $8 \times 8$ SiPMs. Thereafter, the $\mathrm{Z}$ coordinate was determined using the estimator described as the ratio of the impact energy to the SiPM row (or column) to the highest signal [20]. The energy of each event is simply extracted by the sum of all channels fired.

For optimum timing determination, we have investigated an offline positioning filter. This means that an event is valid as long as the channels fired are in adjacent SiPMs (maximum of 8 therefore). In that way, false triggering due to SiPM dark counts can be rejected. 


\subsection{Timing linearity tests}

The timing linearity of the system was studied with two detectors at a fixed distance, while the ${ }^{22} \mathrm{Na}$ source was moved across the field of view in between them. This experiment was carried out using the two monolithic blocks. We recorded the centroid of the timing distributions and compared the linearity observed from the measured experimental centroids and the expected values.

\section{Results}

\subsection{Pixelated crystals: one-to-one coupling}

The experiments with the KETEK PA3325 SiPMs and the small crystal pixels showed a CTR of 186 ps FWHM using default ASIC thresholds. Both detectors showed an energy resolution near $10.8 \%$ after correction for the SiPM saturation. Fig. 3 top shows both the energy plot and CTR histogram. The measurement was carried out at $19^{\circ} \mathrm{C}$. The tests were repeated using the $6 \times 6 \mathrm{~mm}^{2}$ photosensors and LYSO pixels of $6 \times 6 \times 15 \mathrm{~mm}^{3}$. Despite the larger active area of the photosensors that might introduce signal jitter due to larger capacitances, and the thickness of 15 $\mathrm{mm}$ of the LYSO pixels, a timing resolution of 330 ps FWHM was obtained (Fig. 3 bottom). For this set-up, the energy resolution was found to be $13.7 \%$ after again applying an energy calibration.
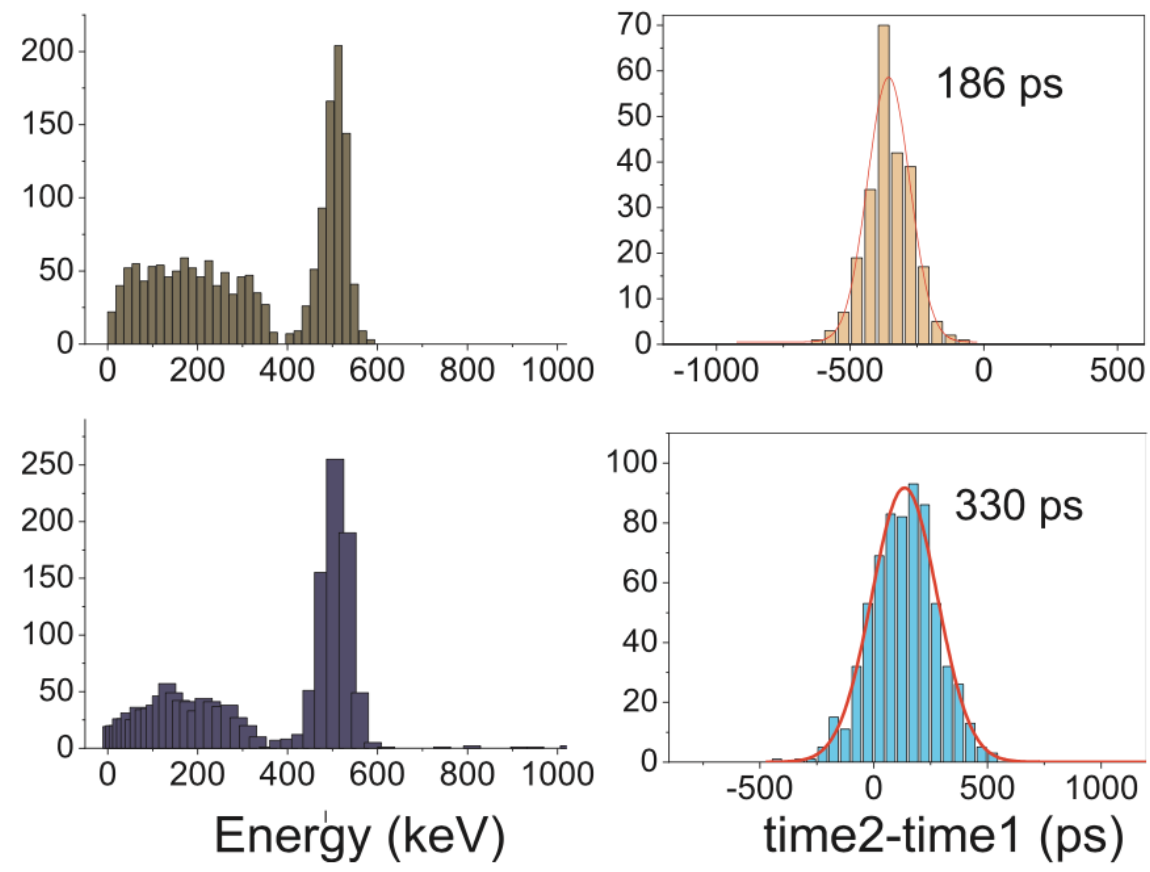

Figure 3. Top, energy spectrum after energy calibration of one detector and time distribution obtained with $3 \mathrm{~mm}$ SiPMs and LYSO crystals of $3 \times 3 \times 5 \mathrm{~mm}^{3}$. Bottom, energy spectrum (after calibration) of one detector and time distribution obtained with $6 \mathrm{~mm} \mathrm{SiPMs}$ and LYSO crystals of $6 \times 6 \times 15 \mathrm{~mm}^{3}$. 
The small size source was placed right in front of the reference detector and, therefore, the whole area of the monolithic crystal was irradiated during the coincidence measurements. An energy profile of all events in the monolithic crystal is shown in Fig. 4 top-left. Events within the photopeak (30-48 ADC units) were selected for data analysis. Three different Regions of Interests (ROIs) at the corner, middle and center of the detector block, were selected by applying a position filter, as depicted in Fig. 4 bottom-left. Moreover, for each ROI, the DOI distribution of events was obtained, allowing us to further split the data in three DOI regions (about $5 \mathrm{~mm}$ each) depending on the gamma ray impact $\mathrm{Z}$ coordinate. They are named as DOI1 for events at the crystal entrance, DOI2 for events occurring at the middle of the scintillator and DOI3 for events impinging at the bottom crystal layer (see Fig. 4 top-right). Therefore, an estimation of the average number of channels that crossed the threshold and, hence, of the SNR per channel could be obtained for each gamma-ray impact. As seen in Fig. 4 bottom-right, we observed that independently of the XY position, a larger spread of the scintillation light was found for events at the upper crystal layers (DOI1). For impacts impinging deeper in the monolithic crystal e.g. DOI2 and DOI3, we can observe a slightly decreased in the number of channels fired, but still high suggesting a poor SNR per ASIC channel.
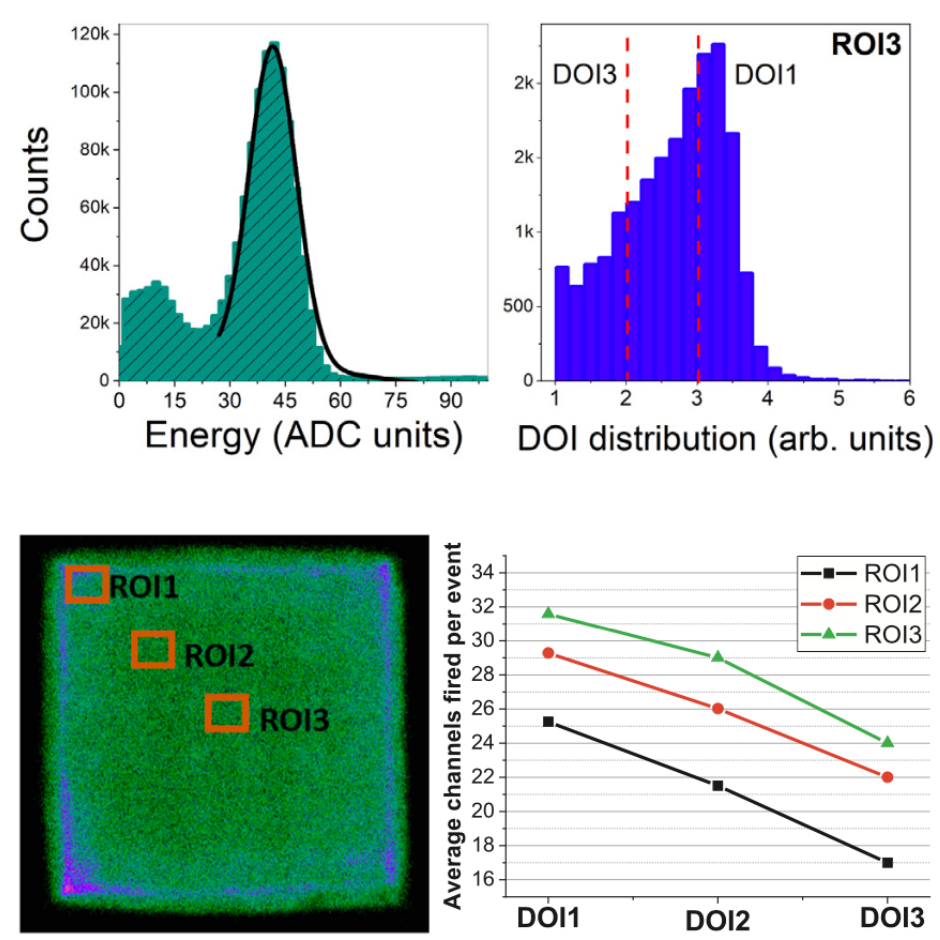

Figure 4. Top-left, energy spectrum of the whole monolithic based detector before calibration. The black line shows a fit to the distribution using a Gaussians profile plus a line. Top-right, DOI distribution of the events recorded at the center of the monolithic crystal (ROI3). Bottom-left, flood map of events, showing the three ROI selected for analysis. Bottom-right, average number of channels fired per event, as a function of the DOI and for the three ROIs. 
A significant dependency of the number of fired channels with the gamma-ray impact position is observed. The nearest an event occurred to the edge of the crystal, the more it suffers from light truncation as a high amount of scintillation photons are absorbed by the black painted walls. This fact explains the decreased number of channels fired for ROI1 and ROI2. It should be noticed, that these distributions are in general directly related to the dimensions and thickness of the crystal block as well as to the crystal treatment and photosensor geometry.

We have shown that the generated SNR per photosensor element strongly depends on the position of each particular event. Since an average of 25 channels are fired for each gamma-ray event, a poor SNR in the ASIC channels is expected. Gamma-ray impacts near the crystal entrance (DOI1), which is the most probable scenario, will fire many photosensors but with a reduced number of collected scintillation photons per photosensor. This statement limits the basic TOF requirements, namely a short and sharp rise time of the signals [12]. On the contrary, events near the photosensor show a narrower light spread (DOI3), permitting a faster and a more efficient collection of optical photons. We shorted all impacts based on their timestamps and we used this information to fill the histograms shown in Fig. 5 top. Earliest hit 0 (X-axis of the histogram) means that the first timestamp also collected the maximum number of optical photons. Whereas for instance, hit labelled 10 means that the 10th impact collected the highest energy for this given gamma-ray event. Therefore, for gamma-ray impacts near the photosensor (DOI3), the channels collecting the highest amount of energy also correspond to the fastest ones (first hits). That is, we observe the hits with highest energy being the earliest collected. However, impacts at the crystal entrance exhibit a wider distribution of energy hits and time. This fact, was found to be directly related with the timing resolution.
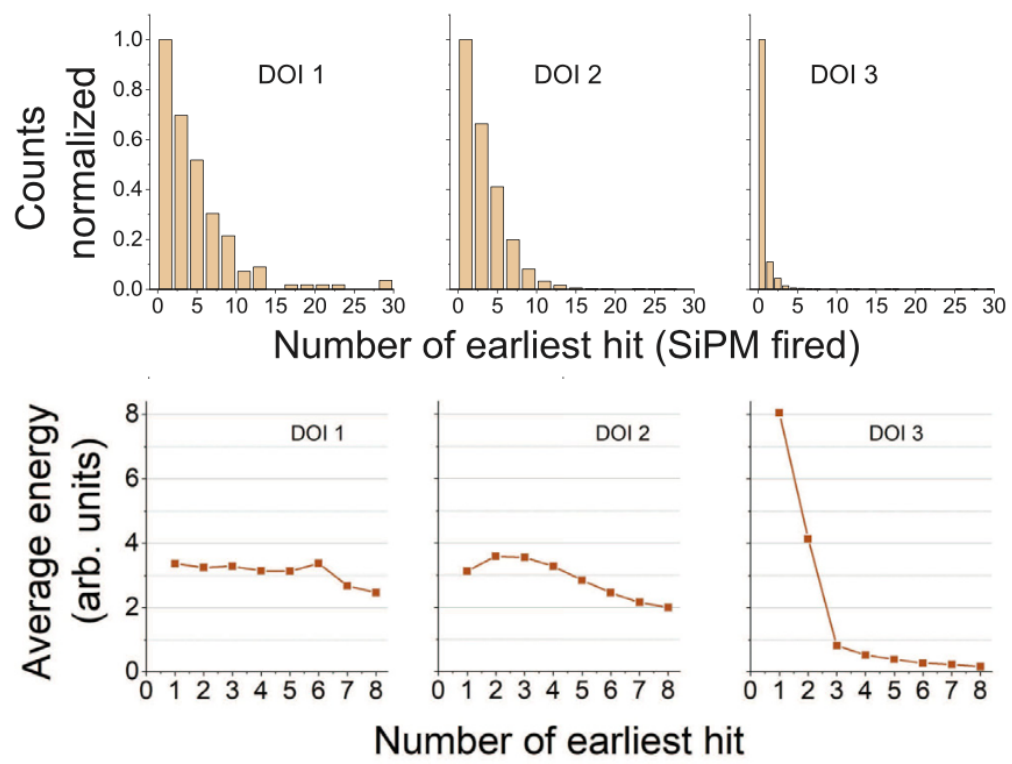

Figure 5. Top, histograms showing which of hits collected the highest amount of energy for the three DOI regions for the whole scintillator. Bottom, average energy of each hit for all events recorded at the three DOI layers (no filter in position). 
Also interesting is the analysis of the energy ranges of the earliest channel triggered (earliest timestamp recorded) which complements the previously described behavior. By averaging the energies of the eight earliest hits for all events, it was clearly shown that the first recorded hit shows much higher energy ranges compared with the later recorded ones for the case of deep DOIs, while at the higher DOI1, the energy ranges for all 8 first hits are all comparable (Fig. 5 bottom). It should be noted, that these plots were obtained for the whole scintillator volume without using the previously described position filter. No significant variations are expected in these distributions for independent ROIs.

The variations in the spread of the scintillation light depending on the DOI of each gamma event led to explore the optimal event timestamp assignment method [11]. When that many hits occurred for each event, it is critical to study if the optimal time resolution is given when using the first timestamp recorded of each event or an alternative approach is may needed.

\subsection{Monolithic detectors, time analysis}

When using the monolithic crystal and the reference pixel, the assembly was placed at a stable temperature environment of $7^{\circ} \mathrm{C}$, minimizing dark count rates and increasing the Photon Detection Efficiency (PDE) of the photosensors. Coincidences measurements were carried out with the ${ }^{22} \mathrm{Na}$ source attached to the reference detector and data for the whole scintillator volume were obtained.

We first obtained the timing resolution using the timestamp of the channel with the highest energy, resulting on $1.41 \mathrm{~ns}$ FWHM. Alternatively, we sorted the data based on the timestamp and we used the earliest one recorded for the timing distribution. By plotting the difference of the timestamps, we observed an additional satellite peak centered at 5000 ps, see Fig. 6 top. The satellite peak is directly related to the overvoltage of the SiPMs as well as with the value of the vth_t1 discriminator. Detailed analysis of this effect can be found in [28].

We applied a timing filter window accepting events whose first several hits recorded are within a time frame. In particular six hits were chosen as the optimum number of hits within this window. This filter had as a result an improvement of the CTR and the discard the satellite peak from the timing distribution plots, showing that this effect was a result of false triggering (Fig. 6 bottom). Table I summarizes the measured CTR for different filter timing windows. As it can be seen, narrower time windows significantly improve the CTR but also affect the statistics. Therefore, a window of 2 ns was selected and applied to all following measurements. This filter improved the measured time resolution to 996 ps FWHM. 


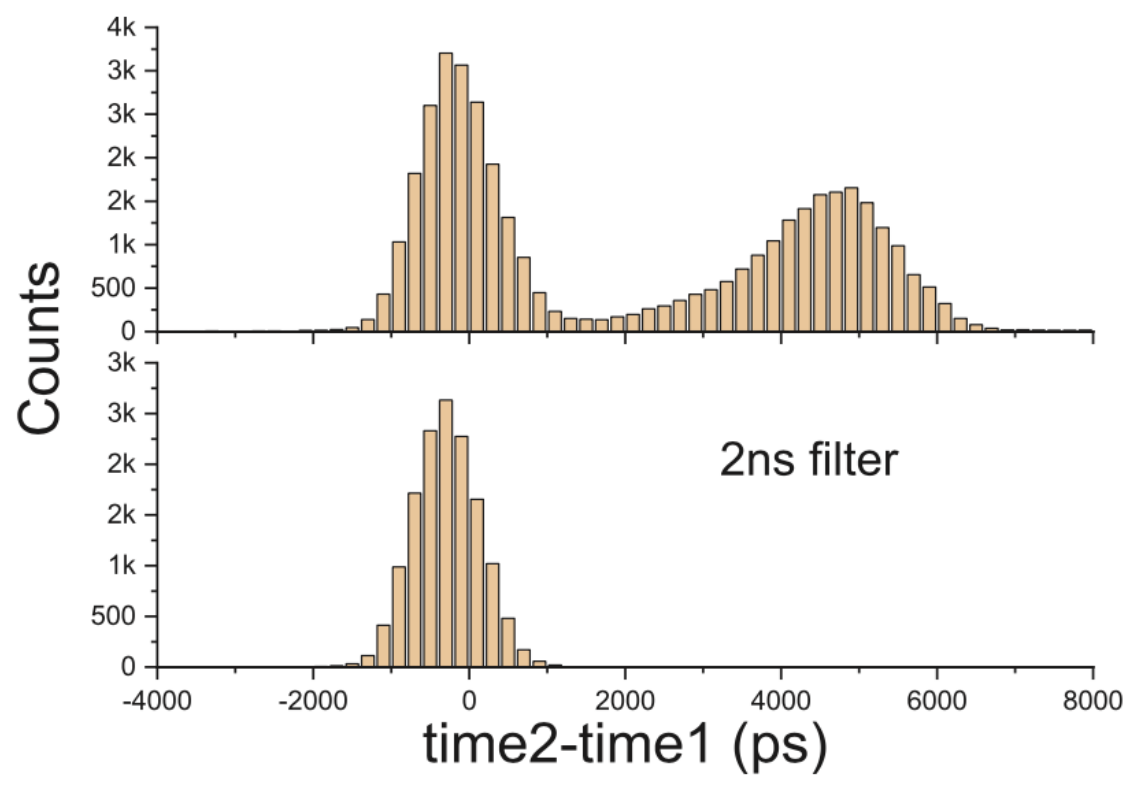

Figure 6. Top, timing distribution of the measurement between the monolithic block and the reference detector without applying filtering windows. Bottom, timing distribution when applying a 2 ns window for the first six impacts.

\begin{tabular}{|c|c|}
\hline Filtering Window (ps) & $\begin{array}{c}\text { CTR } \\
\text { (FWHM, ps) }\end{array}$ \\
\hline 5000 & 1156 \\
\hline 4000 & 1133 \\
\hline 3000 & 1100 \\
\hline 2000 & 996 \\
\hline 1000 & 817 \\
\hline
\end{tabular}

Table I. Table representing the CRT values as well as the statistics of the total event accepted for different filtering windows of the first 6 hits.

Some authors have showed a significant CTR improvement when instead of the timestamp of the first hit, the timestamps of secondary hits are considered together with a low threshold at the level of the first photo-electron [29][30]. Figure 7 shows experimentally the same behavior. When using the timestamp of the fourth recorded hit in time, the time resolution was improved. Herein, using this approach and the fourth arrived timestamp, we were able to reach to an improved CTR from 996 ps (RAW data) to 883 ps FWHM. 


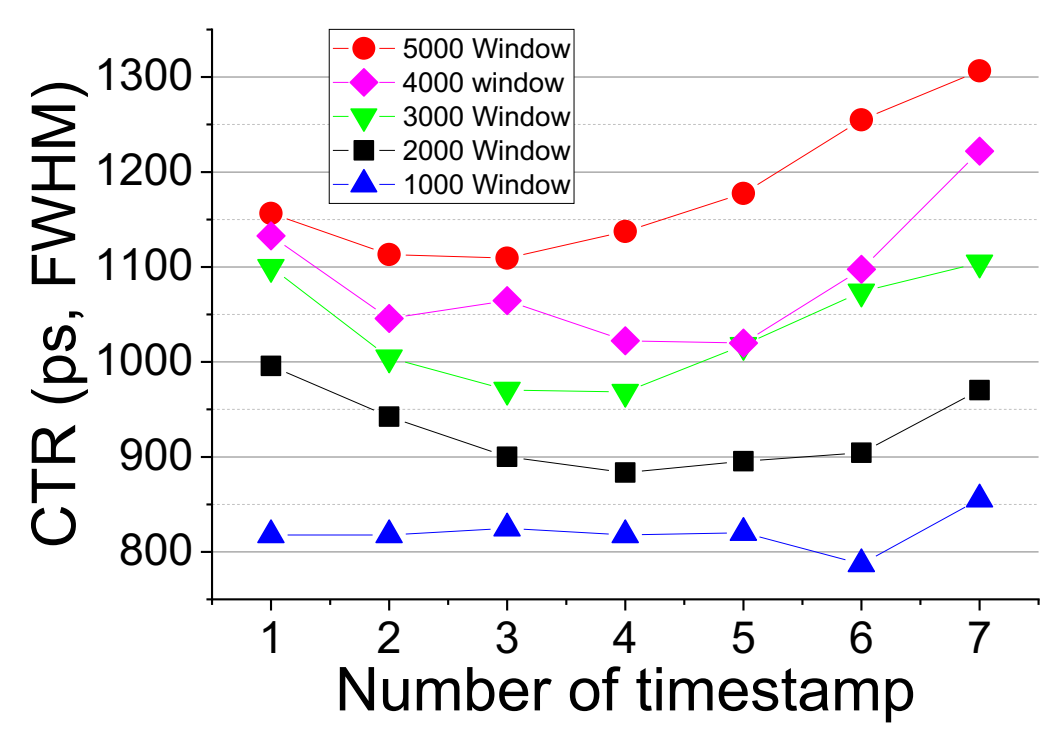

Figure 7. Experimental results showing CRT measured as a function of the number of earliest timestamp used for different filtering windows.

The timing resolution measured for this set-up is still influenced by the time-skew among the ASIC channels. Moreover, the time-walk also affects the CTR due to the poor collection of photons per photosensor element. Thus, a slower rising time is observed as a consequence of the scintillation light sharing effect.

\subsection{Time-skew and time-walk calibration}

The reference detector with the single LYSO pixel was placed at a distance of $25 \mathrm{~cm}$ from the monolithic detector and measured in coincidence mode. The ${ }^{22} \mathrm{Na}$ point source was attached to the reference detector aiming again to irradiate the whole volume of the crystal block and about 106 events were recorded. Considering that the source, as well as the distance between detectors, remained constant during the experiment, the mean values of the timing distributions of all timestamp differences between all channels in the monolithic block and the reference one, should ideally be constant, independently of the energy collected.

Initially, aiming to obtain an estimation of the time-skew error, and not for calibration purposes, we selected events which occurred at the bottom of the crystal block and whose earliest recorded impacts contain a relatively high number of photons (8 ADC units). This filter was applied in order to consider only timestamps that are less influenced from noise. The aforementioned Gaussian mean values for the 64 pairs were obtained. These represent the timeskew for the 64 ASIC channels. Figure 8 depicts the time offsets for all ASIC channels in this assembly. The introduced error can be as large as $1 \mathrm{~ns}$ when considering all channels for the CTR estimation. 


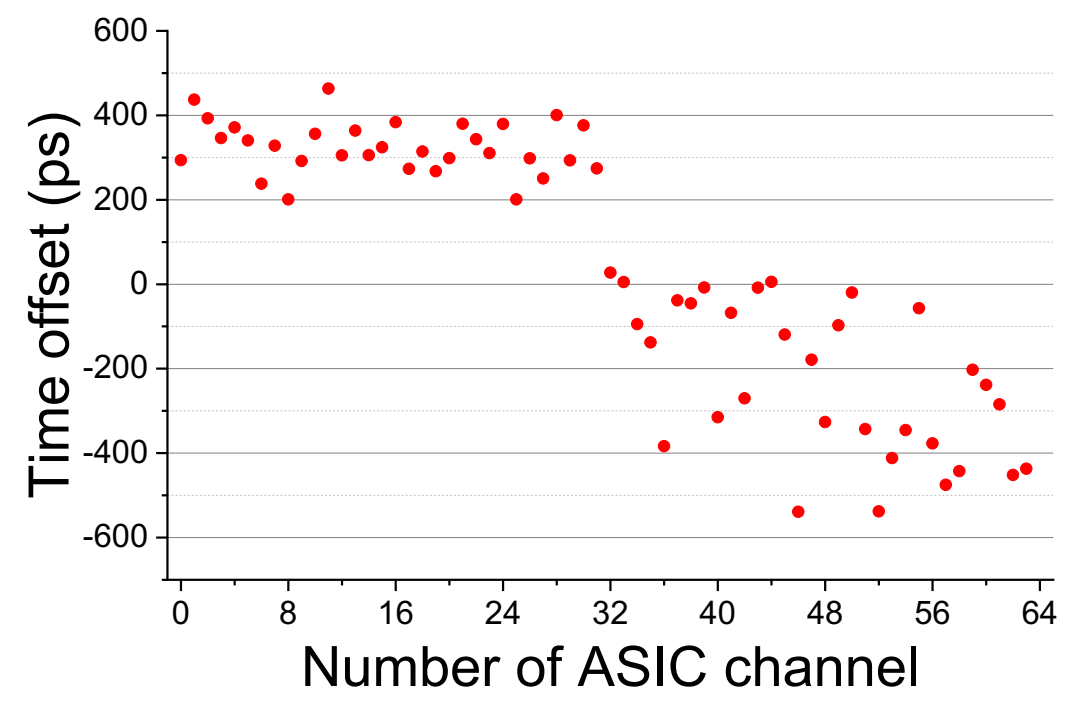

Figure 8. Dispersion of Gaussian centroids of the time differences between the channels of the monolithic detector and the reference one (time-skew error).

In the following we describe the studies carried out regarding the time-walk influence. Figure 9 left shows the timestamps differences for one single pair of channels as a function of the energy of the first hit recorded in the monolithic block before any calibration. Even when considering one single channel the timing resolution is strongly affected for lower energy impacts (see range 0 to 10 in arbitrary units), confirming the time-walk effect.

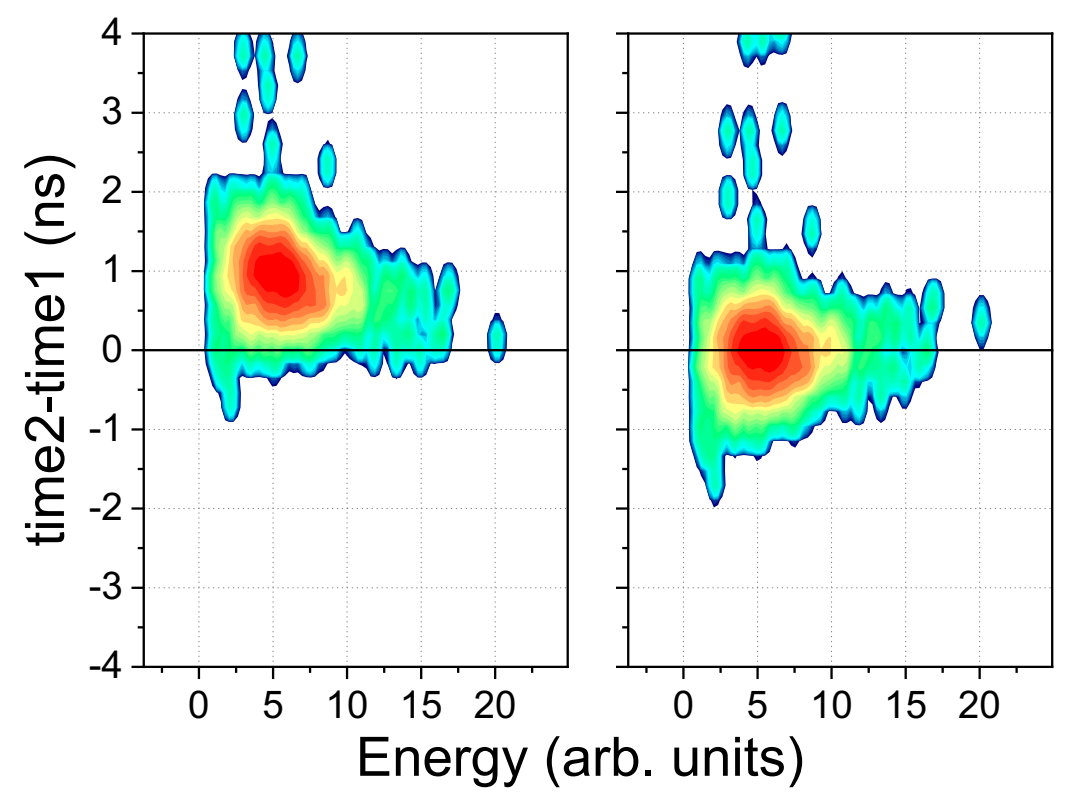

Figure 9. Time differences of one channel as a function of the energy of the first hit before (left) and after the calibration (right). The color map is in logarithmic scale.

The 2D histograms containing the time differences as a function of the energy were generated for each channel of the monolithic detector (a total of 64). Then, they were fitted using a parabolic 
function and the fitting parameters stored in a Look-Up-Table. A parabolic function was used as it agrees well with the data behavior. The application of this method to all 64 channels of the monolithic based detector, besides some partial time-walk correction, also accounted for the timeskew errors, as all channel distributions were centered to zero (see Fig. 9 right). After correcting each recorded timestamp, an improvement of the CTR was observed for all channels, with the average value to be 851 ps FWHM, when using the earliest recorded timestamp.

The time arrival of secondary hits was again studied in detail, after applying all timestamp corrections. Figure 10 shows the CTR when later recorded timestamps were used. A slighter improvement was observed when the second hit was used (black squares). However, we have also investigated the averaging of the timestamps $\left(t_{i}\right)$ of the few first hits and not just considering one. We have tested both a simple averaging of timestamps $\left(t_{\mathrm{SA}}\right)$ as well as an energy weighted average $\left(t_{\mathrm{EA}}\right)$ :

$$
t_{S A}=\frac{\sum_{i=0}^{n} t_{i}}{n}, \quad t_{E A} \frac{\sum_{i=0}^{n} t_{i} E_{i}}{\sum_{i=0}^{n} E_{i}}
$$

Up to eight timestamps were considered for both methods, see also Fig. 10, blue triangles $\left(t_{\mathrm{SA}}\right)$ and red circles $\left(\mathrm{t}_{\mathrm{EA}}\right)$, respectively. In particular, slightly optimum values were provided by the energy weighted average when the six earliest timestamps recorded were used, reaching about 580 ps FWHM. These values have been obtained for the whole monolithic crystal volume.

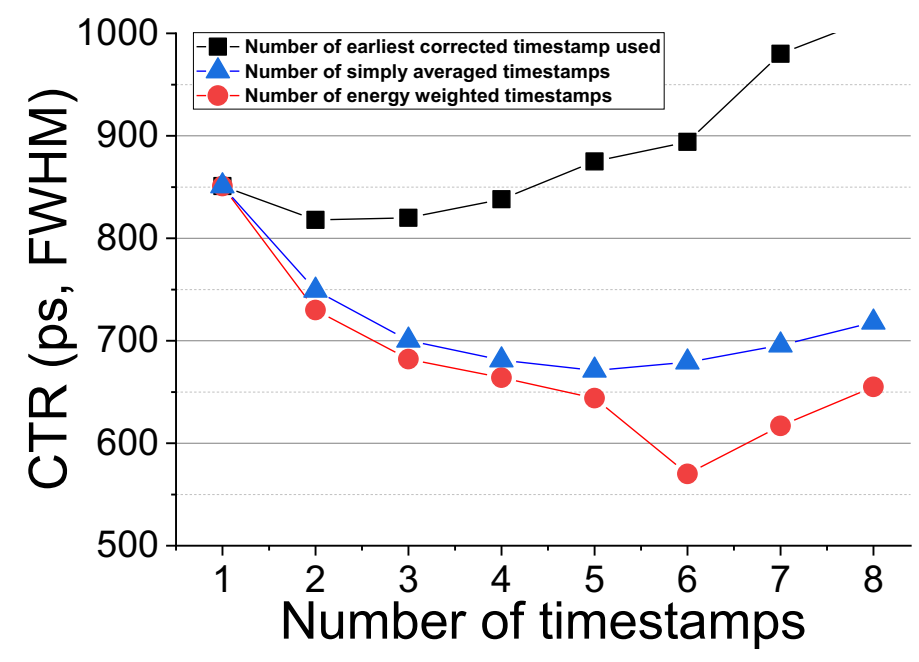

Figure 10. Experimental CTR results (not position filter applied) using three methods; considering individually the eighth earliest timestamps (black squares), a simple average of timestamps (blue triangles) and an energy weighted averaging (red circles).

Finally, after enabling the position filter mentioned in section 2.4, an additional improvement in the CTR was found. The CTR improved from 580 ps to 550 ps FWHM. Since the contribution of the reference detector was estimated at $235 \mathrm{ps}$ FWHM (330/ $\sqrt{2} \mathrm{ps})$, the resulting time resolution for the monolithic based detector was found to be 497 ps FWHM. 


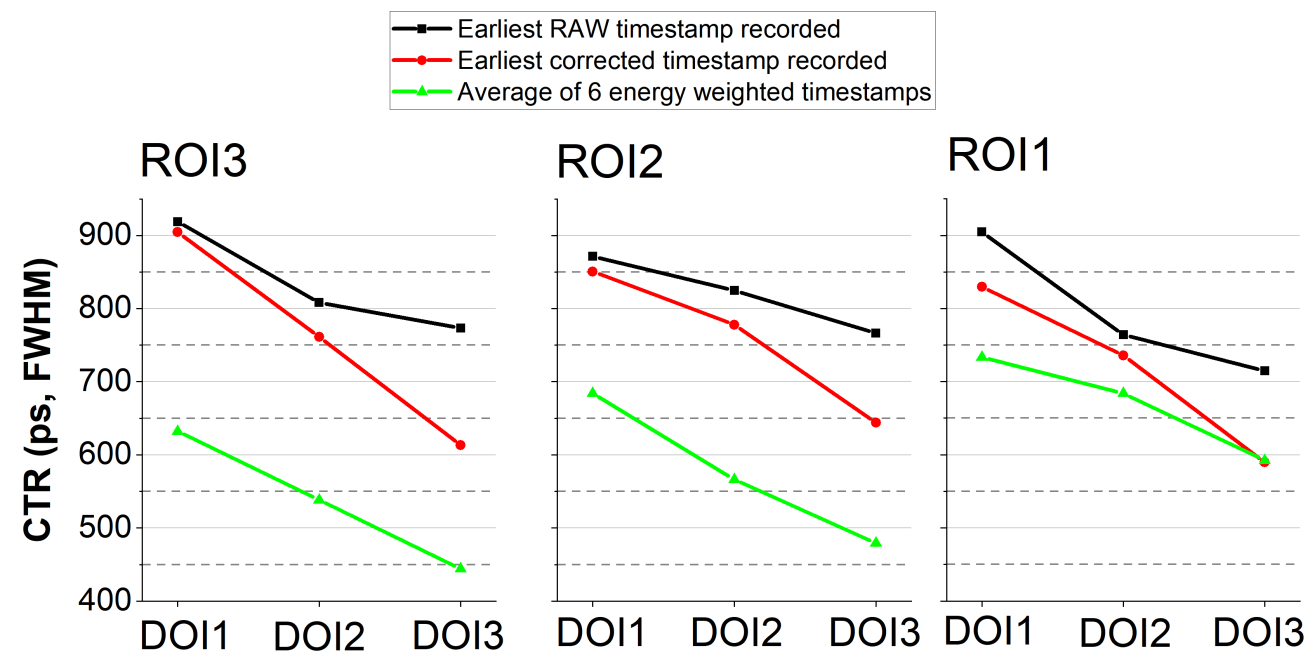

Figure 11. Timing resolution CTR as a function of the DOI layer for the three ROIs, when using RAW timestamps, the earliest recorded timestamp corrected, and an energy weighted average of the earliest 6 corrected timestamps.

The three ROIs showed in section 3.2 were selected for an independent and in deep detail analysis of the CTR performance based on the $\mathrm{X}, \mathrm{Y}$ and $\mathrm{Z}$ position of the gamma-ray event. In all three ROIs when RAW timestamps are considered, the influence from the time walk and the poor SNR significantly affects the time resolution. This is especially observed for impacts at the crystal entrance layer (DOI1), as depicted in Fig. 11 with black squares. The same dependency, CTR vs DOI layer, is also found when only one corrected timestamp is used, but with some CTR improvement, as expected. However, when additional timestamps (six of them) are averaged using an energy weighting method the CTR is highly improved (green squares) and most importantly, its dependency with the DOI layer significantly decreases. Moreover, it is worth to highlight that in the case of events occurring near the crystal corner (ROI1) and at the bottom crystal layer (DOI3), the averaging method of timestamps seems to provide very similar results to the case of using just the earliest corrected timestamp. This behavior can be expected from the fact that firstly, as already showed in Fig. 4, most of the collected scintillation photons occur in the first hit. Moreover, the scintillation light absorption by the black painted laterals limits the light spread. Besides this, no significant variations in the timing behavior were found among ROIs, however best values were obtained for the case of the ROI3 and DOI3, resulting in a CTR value of 440 ps FWHM (371 ps FWHM when subtracting the contribution of the reference detector).

\subsection{Experiments with two monolithic blocks}

The two monolithic blocks were independently calibrated using the approach described above with a reference single-pixel detector. Then, they were measured in coincidence by placing the source in between the two detectors. Figure 12 top shows the CTR values when considering an average of up to 8 timestamps (energy and simply average). Best timing resolution was achieved 
at 660 ps FWHM when using the sixth earliest timestamps weighted by energy. This data includes all impacts in the whole scintillation volume.

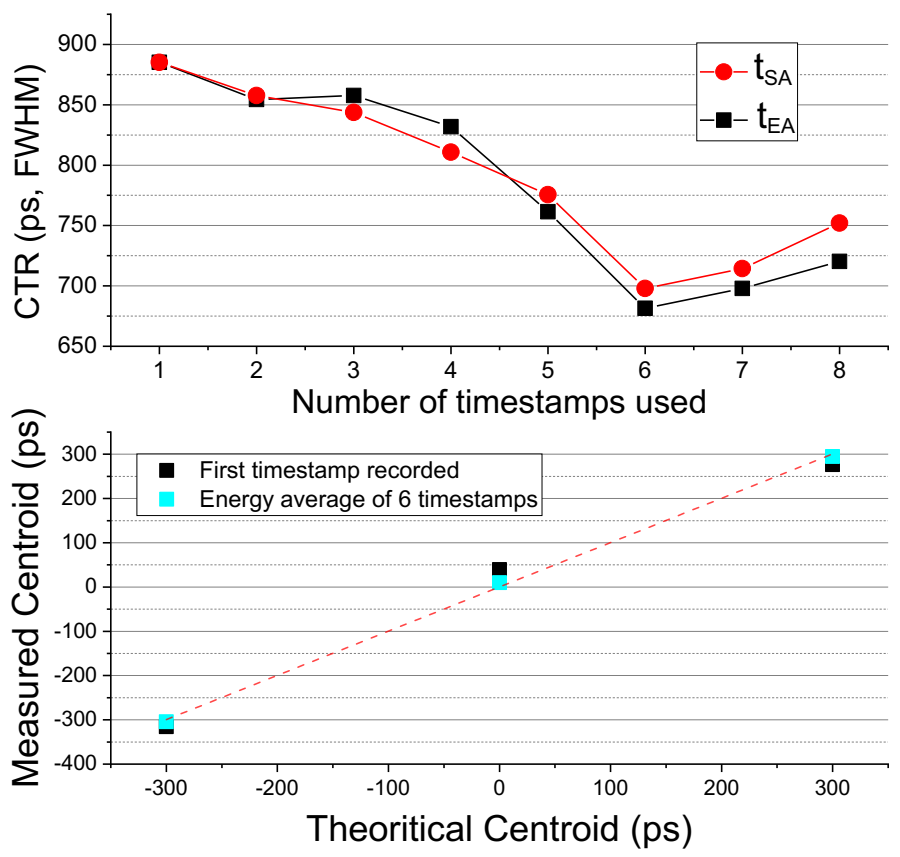

Figure 12. Top, timing resolution of the coincidence measurement between the two monolithic detectors using simple timestamps average (red circles) and energy weighted timestamps average (black squares). Bottom, measured centroids as a function of the theoretical expected centroids using the earliest corrected timestamp (black squares) and an energy average of the 6 earliest timestamps (cyan squares).

In order to validate the timing results, the linearity of the measured Gaussian centroids of the timing distributions was evaluated. In figure 12 bottom, the centroids obtained with the use of just one corrected timestamp as well as with the average of 6 timestamps weighted by the energy are plotted against the theoretical ones. Results shown that when using the first timestamp (black squares) a regression coefficient of 0.97 was obtained, while with the averaging method this was improved to 0.99 (cyan squares).

\section{Discussion}

The TOFPET2 ASIC is capable of resolving, with high accuracy, the gamma impacts in terms of timing and energy resolution. The experiments carried out with the small LYSO pixels $(5 \mathrm{~mm}$ thick) exhibited state-of-the-art CTR values of 186 ps FWHM using commercially available electronics. We hardly faced drawbacks reaching this good timing, at even $19{ }^{\circ} \mathrm{C}$ set-up temperature. However, when using thicker crystal pixels $(15 \mathrm{~mm})$ and larger photosensors of $6 \mathrm{~mm}$ size, a deterioration to 330 ps was observed, as expected.

The use of a single pixel reference detector in the experiments with the monolithic crystals, permitted a better understanding of their timing performance. Here, the small crystal pixel minimizes the error introduced to the CTR determination. 
An energy filter was applied to all measurements selecting events within the $511 \mathrm{keV}$ photopeak. Regarding the energy resolution in the monolithic based detector, it was found to be nearing 30\% when considering all events within the whole crystal volume. However, this significantly improves when selecting small ROIs. For instance, for a ROI at the crystal center, an energy resolution of $17 \%$ was determined. The poor resolution obtained when considering all events independently of the position of the event, is caused by the effect of scintillation light truncation at the crystal edges. Moreover, the very low thresholds might produce some small deterioration in the energy resolution.

A parameter of significant importance on the timing resolution of the monolithic block was found to be the timestamp assignment. For comparison, in the case of pixelated crystals and the one-to-one coupling, the best CTR results are seen when using the timestamp of the hit with the highest energy, if more than one photosensor is fired (not shown in this work). However, this is no longer the case for monolithic based detectors. If the events occur near the photosensor, without producing any light sharing, the optimal approach would be to assign the timestamp of the first hit recorded, which is also the hit with the highest number of photons (energy) collected. However, in the case of events occurring in the entrance regions of the crystal, this approach cannot be applied, due to the low collection probability of enough photo-electrons in just one channel.

When there is an intense scintillation light sharing, very low thresholds are needed making the electronics sensitive to the very first photo-electrons. However, as we showed in this work, this low threshold may result in the acceptance of false triggered events, introducing uncertainty in the timing distribution. The timing window filter allowed us to discard noisy events and to improve the CTR. Although this filter significantly reduced the acquired statistics, we expect most of those rejected events are a result of false triggering. Moreover, by operating at very lower temperatures, a further reduction of the dark count rate will be achieved and, thus, discarding a smaller number of events. It should also be noted that the improvement seen by applying this filter is not related to the time-skew error as the improvement is also seen when only single pairs of channels are considered for the timing distribution, a method in which the time-skew has not effect.

It was observed that secondary hits provide better results in terms of timing. In some cases, an improvement better than 100 ps FWHM was observed. This behavior has been studied in depth elsewhere and is directly related to the order and photocounting statistics [30]. As previous works have shown, when the optical photon index (i.e. hit number) increases, the time interval among the following detected photons is reduced. This means the probability distribution for detecting the first photon is significantly larger compared to the probability of determining the detection time of secondary photons. Therefore, it is hypothesized that the improvement seen in the experimental results is based on the timing generation probability of photons and in the order 
statistics theory, since there was no relation with the energy of each hit (as shown in Fig. 5 bottom).

A calibration procedure was carried out to correct each timestamp. We generated 2D plots of the time differences for each channel pair as a function of the impact energy. Here, we studied the time given using the timestamp of the first hit recorded, of the first 8 hits or of all hits. However, not significant differences were found among them, so the first hit was decided to be used in these plots. Instead of applying fits to the 2D plots (time difference vs. energy) for each channel, another method was formerly studied [26]. In that case, projections into the time difference axis were made in small energy steps. The centroids of the Gaussian-like profiles were used as timestamps offsets. However, since lower timing thresholds were currently used, the fitting approach described in this work showed slightly more accurate results.

Even after the calibration, the effect of the time-walk as well as of the false triggering might still be present. We expect some uncertainties in the generated timestamps, especially when the channel triggered did not collect a significant amount of scintillation photons. However, the method of averaging several timestamps, and in particular when weighting them by their collected energy, showed a significant improvement of the CTR, as it minimized the contribution of the noisy generated timestamps. This fact, was also verified when independently treating the CTR for different planar and DOI regions. As it was shown, the timestamp averaging method lead both to a partial compensation of the CTR and light spread dependency, providing more accurate CTR results than a single timestamp approach. The only difference was observed in the case of events occurring near the crystal edge and at the DOI layer near the photosensors. Here, we measured similar CTR both when using the averaging method or just a single timestamp. This might be explained due to the limited light spread in this region of the crystal. Herein, by analyzing the CTR as a function of the DOI we were able to study the timing performance while avoiding the uncertainty introduced by the light speed propagation.

The CTR values obtained for the two monolithic blocks assembly were in accordance with the CTR recorded for the monolithic block in coincidence with the single pixel detector. We have estimated combined statistical and systematic error bars of about 20-30 ps FWHM. Notice that the custom PCB developed to interface the J-Series photosensors with the ASIC readout might introduce some additional noise to the signals coming from the photosensors due to their signals time-paths and higher capacitance. The validation of all methods used and described in the present manuscript was achieved with the linearity of the Gaussian centroids of three space-separated measurements.

\section{Conclusions}

We have evaluated the TOFPET2 ASIC showing its capability to achieve sub-200 ps FWHM time resolution using crystal pixels. 
A thick and wide monolithic block was selected to be tested and explored in terms of timing resolution. The volume of the selected scintillation block exhibited several challenges in the determination of an accurate impact time resolution. The light sharing effect, and the resulting poor SNR per ASIC channel, is related to the size of the monolithic block. In addition to this, the selected treatment (black lateral paint and retroreflector layer at the entrance), on one hand enhances the determination of the impact coordinates, but on the other hand significantly degrades the timing resolution due to the scintillation light absorption at the lateral walls. We are aware these components somehow constrained the achieved performance, and that better absolute values could be obtained using smaller monolithic blocks, with white or reflecting painting, as well as when combined with photosensor arrays with smaller SiPM area. However, the analysis shown in this work is still useful to understand the overall limits and corrections to be applied when using monolithic blocks read out using analog SiPMs and ASICs. We have added especial focus in this work to the time-walk and the time-skew corrections.

The time-skew can be addressed through the independent processing of channel pairs but in the case of the monolithic block, the presence of time-walk uncertainties produces additional difficulties when aiming for an accurate calibration. Nonetheless, the calibration method described in this work provides good results. The time-skew was successfully corrected, permitting the exploitation of the timing information during future reconstruction processes. In addition, the time-walk has also been partially compensated, a fact that permits and motivates a follow up research work towards the development of TOF-PET detectors using other types and treatments of monolithic blocks.

Summarizing, RAW timing resolutions were found to be well above $1 \mathrm{~ns}$ for a large $50 \times 50 \times 15 \mathrm{~mm}^{3}$ LYSO block when tested in coincidence against a reference pixel-based detector. Techniques to discard a fraction of noisy events and decrease the time uncertainty were applied, reaching a significant improvement in terms of CTR of 550 ps FWHM for the whole scintillation volume, without subtracting the reference detector contribution which is estimated at $230 \mathrm{ps}$ FWHM. As shown in the analysis of the CTR and event position dependency, an improved timing resolution can be achieved for events at the center of the crystal and deep DOIs layers of $440 \mathrm{ps}$ FWHM (again without subtracting the reference detector contribution). When two identical detectors were tested, CTR values of 660 ps FWHM were found. This timing resolution clearly cannot permit the use of timing information in the lines of response for small or organ dedicated systems [26], but will permit the reduction of noise as well as the improvement of the SNR in the reconstructed images. Moreover, recent pilot studies in our lab have shown to improve these results up to a factor of 2 if smaller crystals ( $1 \times 1$ inches), Teflon wrapped, and coupled to $8 \times 8$ SiPM arrays $\left(3 \times 3 \mathrm{~mm}^{2}\right)$ are used. 


\section{References}

[1] W. H. Wong et al., Image improvement and design optimization of the Time-of-Flight PET. J Nucl Med. 24 (1983), 52-60.

[2] R. Gariod et al., The LETI Positron tomograph architecture and time of flight improvements. Proceeding of the Workshop on Time-of-Flight tomography. IEEE Publication: Washington University (1982), 25-29.

[3] W. H. Wong et al., Performance characteristics of the University of Texas TOFPET-I PET camera. J Nucl Med. 25 (1984), 46-47.

[4] S. Surti, Update on time-of-flight PET imaging. J Nucl Med. 56:1 (2015), 98-105

[5] P. Lecoq, Pushing the limits in Time-Of-Flight PET imaging. IEEE Transactions on Radiation and Plasma Medical Sciences. 1 (2017), 473-485.

[6] V. C. Spanoudaki and C. S. Levin, Photo-detectors for time of flight positron emission tomography (ToF-PET). Sensors. 10 (2010), 10484-10505.

[7] T. Szczęśniak et al., Fast Photomultipliers for TOF PET. IEEE Trans. Nucl. Sci. 56 (2009), 173-181.

[8] D. Renker, New trends on photodetectors. Nucl. Instr. Meth. Phys. Res. A. 57 (2007), $1-6$.

[9] C. Kim et al., Multi-Pixel Photon Counters for TOF PET Detector and Its Challenges. IEEE Trans. Nucl. Sci. 56 (2009), 2580-2585.

[10] T. Frach et al., The digital silicon photomultiplier - System architecture and performance evaluation. IEEE Nuclear Science Symposuim \& Medical Imaging Conference, Knoxville, TN, 2010, 1722-1727.

[11] T. Frach et al., The digital silicon photomultiplier - Principle of operation and intrinsic detector performance. 2009 IEEE Nuclear Science Symposium Conference Record (NSS/MIC), Orlando, FL, 2009, 1959-1965

[12] W. W. Moses, Current trends in scintillator detectors and materials. Nucl Instrum Meth A. 487 (2002), 123.

[13] S. Gundacker et al., Measurement of intrinsic rise times for various L(Y)SO and LuAG scintillators with a general study of prompt photons to achieve 10 ps. Phys Med Biol. 61:7 (2016), 2802-37.

[14] S. Gundacker et al., State of the art timing in TOF-PET detectors with LuAG, GAGG and L(Y)SO scintillators of various sizes coupled to FBK-SiPMs. JINST, 11 (2016), P08008.

[15] S. Surti and J. S. Karp, Advances in time-of-flight PET. Phys Med 32:1(2016), 12-22.

[16] S. Gundacker et al., Time resolution deterioration with increasing crystal length in a TOF-PET system. Nucl Instr Methods. 737 (2014), 9. 
[17] R. Marcinkowski et al., Optimized light sharing for high-resolution TOF PET detector based on digital silicon photomultipliers. Physics in medicine and biology. Phys Med Biol. 59:23 (2014), 7125-39.

[18] A. González-Montoro et al., Detector block performance based on a monolithic LYSO crystal using a novel signal multiplexing method. Nucl Instr Methods. 912 (2018), 372377.

[19] D. Xi et al., Optimization of the SiPM Pixel Size for a Monolithic PET Detector. Physics Procedia. 37 (2012), 1497-150.

[20] A. Gonzalez-Montoro et al., Performance Study of a Large Monolithic LYSO PET Detector With Accurate Photon DOI Using Retroreflector Layers. IEEE TRPMS. 1 (2017), 229,237).

[21] S. Krishnamoorthy et al., Performance evaluation of the MOLECUBES $\beta$-CUBE - A high spatial resolution and high sensitivity small animal PET scanner utilizing monolithic LYSO scintillation detectors. Phys Med Biol. 63:15 (2018), 155013.

[22] A. González-Montoro et al., Novel method to measure the intrinsic spatial resolution in PET detectors based on monolithic crystals. Nucl Instr Methods. 920 (2019), 58-67.

[23] H. T van Dam et al., Sub-200 ps CRT in monolithic scintillator PET detectors using digital SiPM arrays and maximum likelihood interaction time estimation. Phys Med Biol. 58:10 (2013), 3243-57.

[24] A. Di Francesco et al., TOFPET2: A high-performance ASIC for time and amplitude measurements of SiPM signals in time-of-flight applications. Journal of Instrumentation, 11:3 (2016), C03042.

[25] TOFPET2 ASIC Evaluation kit - Hardware User Guide (v1.2), v1.2, PETsys Electronics SA., 2018.

[26] E. Lamprou et al., PET detector block with accurate 4D capabilities. Nucl Instr Methods. 912 (2018), 132-136.

[27] F. Acerbi and S. Gundacker, Understanding and simulating SiPMs. Nucl Instr Methods. 926 (2019), 16-35.

[28] D. Schug et al., Initial Measurements with the PETsys TOFPET2 ASIC Evaluation Kit and a Characterization of the ASIC TDC IEEE Trans Rad Plasma Med Sci. PP. 1-1. 10.1109/TRPMS.2018.2884564.

[29] S. Seifert et al., A comprehensive model to predict the timing resolution of SiPM-based scintillation detectors: Theory and experimental validation. IEEE Trans. Nucl. Sci. 59 (2012), 190-204.

[30] R. Vinke et al., The lower timing resolution bound for scintillators with non-negligible optical photon transport time in time-of-flight PET. Phys Med Biol. 59:20 (2014, 621529. 
[31] A. J. Gonzalez AJ, F. Sanchez F, J. M. Benlloch, Organ-Dedicated Molecular Imaging Systems. IEEE Trans Rad Plasma Med Sci. 2:5 (2018), 388-403.

\section{Acknowledgments}

This project has received funding from the European Research Council (ERC) under the European Union's Horizon 2020 research and innovation program (grant agreement No 695536). It has also been supported by the Spanish Ministerio de Economía, Industria y Competitividad under Grant TEC2016-79884-C2-1-R. 


\subsection{In-depth evaluation of TOF-PET detectors based on crystal arrays and the TOFPET2 ASIC}

In this contribution we attempt to shed light on the performance of PET detectors based on crystal arrays with pixel sizes different (smaller) than the photosensor active area. As it has already been discussed in the framework of this thesis, this detector approach can result on high spatial resolution capabilities but typically affects the timing performance.

This is a methodological experimental study based on using one particular SiPM type $(8 \times 8$ array, $3 \times 3 \mathrm{~mm}^{2}$ active area) with three different scintillation crystal arrays. Considering that the scintillators were varying only in the total number of crystal elements and in the element size, we were able to recreate different light distributions among SiPMs. This permitted us to obtain a better understanding of the impact in the time resolution of the relative geometrical position between crystal and photosensor elements. In most of the cases, we also made use of calibration and post-processing methods, aiming to improve detector performance. As it is discussed in this contribution, a detailed evaluation in terms of energy, timing and spatial resolution for all detector configurations was carried out. Some of the interesting findings include the capability to resolve all crystal elements, even the smallest ones. Notice that these refer to $1.5 \times 1.5 \times 10 \mathrm{~mm}^{3}$ pixels coupled to $3 \times 3 \mathrm{~mm}^{2} \mathrm{SiPMs}$. In terms of timing resolution, we found a strong dependency of the geometrical coupling between crystal and SiPMs. In several cases, we measured variations in the coincidence time resolution of $130 \mathrm{ps}$ for crystal elements belonging to a single row or column of pixels. The best timing performance was measured for crystal elements that match the SiPM active area, providing a detector time resolution of 156 ps FWHM.

We found this study critical during the course of the $\mathrm{PhD}$ work, given the current evolution of PET instrumentation, demanding everyday more access to TOF information. We envisage the use of crystal arrays configurations in the design of gamma-ray detectors offering both high determination of the planar impact position and precise timing information. We expect the present work will serve as a reference for future design concepts, and will permit a deep understanding of the light sharing effect in crystal arrays. 


\title{
In-depth evaluation of TOF-PET detectors based on crystal arrays and the TOFPET2 ASIC
}

\author{
Efthymios Lamprou, Filomeno Sanchez, Jose M. Benlloch and Antonio J. Gonzalez \\ Published: Nuclear Instruments and Methods in Physics Research A, Volume 977, Pages 164295, 2020. \\ DOI: https://doi.org/10.1016/j.nima.2020.164295
}

\begin{abstract}
In recent years high efforts have been devoted to enhance spatial and temporal resolutions of PET detectors. However, accurately combining these two main features is, in most of the cases, challenging. Typically, a compromise has to be made between the number of readout channels, scintillator type and size, and photosensors arrangement if aiming for a good system performance, while keeping a moderate cost. In this work, we have studied several detector configurations for PET based on a set of $8 \times 8$ Silicon Photomultiplier (SiPMs) of $3 \times 3 \mathrm{~mm}^{2}$ active area, and LYSO crystal arrays with different pixel sizes. An exhaustive evaluation in terms of spatial, energy and timing resolution was made for all detector configurations. In some cases, when using pixel sizes different than SiPM active area, a significant amount of scintillation light may spread among several SiPMs. Therefore, we made use of a calibration method considering the different SiPM timing contributions. Best Detector Time Resolution (DTR) of 156 ps FWHM was measured when using $3 \times 3 \mathrm{~mm}^{2}$ crystal pixels directly coupled to the $3 \times 3 \mathrm{~mm}^{2}$ SiPMs. However, when using $1.5 \mathrm{~mm}$ crystal pixels with the same photosensor array, although we could clearly resolve all crystal pixels, an average DTR of 250 ps FWHM was achieved. We also shed light in this work on the timing dependency of the crystal pixel and photosensor alignment.
\end{abstract}

\section{Introduction}

During the last years, the overall performance of Positron Emission Tomography (PET), including time-of-flight (TOF) technique, has significantly improved, facilitating the diagnostic and therapy assessment processes in several medical fields (e.g. oncology, cardiology, neurology etc.) [1]. The main requirements for a PET system, from the clinical point of view, have been high sensitivity allowing one to significantly reduce the administered dose and/or the scanning time, and good spatial resolution. However, more recently, accurate timing resolution is also feasible, resulting in a highly increased Signal-to-Noise Ratio (SNR) of reconstructed images [1][3]. Those parameters are directly related to the detector configuration. The introduction of Silicon Photomultiplier (SiPMs) along with the rapid progress of electronics and the improvements in scintillation materials have allowed the development of high-resolution PET detectors with accurate timing capabilities [3][4][5].

Several detector configurations have been suggested over the years achieving an excellent spatial resolution. In terms of scintillator geometry, monolithic blocks or crystal arrays have been proposed [6][7]. Indeed, the particular configuration of choice and the crystal type (LYSO, BGO, GAGG, etc...) impact the timing capabilities of the detector block [3]. This dependency is mainly related to the emission and collection of scintillation light [8][9]. In order to reach accurate time 
resolution in PET detectors, a sharp rise time of the scintillation pulse and, thus, a high number of photons in a very short time is mandatory [3][8]. Configurations in which the collection of the scintillation light produced by a single gamma ray is carried out by multiple photosensors (light sharing approaches) typically show a more challenging determination of the impact time tag [8]. On the contrary, in detector configurations where the crystal pixel size is smaller (and centered) or matched to the photosensor element size, typically the best results in time resolution are found. This occurs since most all generated scintillation photons are collected by only one photosensor element with no significant light losses. Unfortunately, if aiming to combine very high spatial and timing resolution using this one-to-one detector approach, a large amount of photosensors and readout channels would be required, meaning a high detector cost.

In this work, crystal arrays of various pixel sizes are evaluated when coupled to the same array of SiPM. This work aims to shed light on different detector configurations, suitable for TOF-PET systems. The current analysis provides a better understanding of the light sharing effect when using crystal arrays, the limitations that show up but also possible ways to compensate them.

\section{Materials and Methods}

\subsection{Scintillators}

Three types of LYSO crystal arrays (EPIC Crystal, Kunshan, China) were used during the experiments, all with $10 \mathrm{~mm}$ thickness but different pixel sizes (Fig. 1). One was composed by $8 \times 8$ crystal pixels of $3 \times 3 \mathrm{~mm}^{2}$ and $3.36 \mathrm{~mm}$ pitch using $\mathrm{BaSO} 4$ as a separator between pixels while Enhanced Specular Reflectors (ESR) of 65 microns, was used on all lateral faces and the entrance (Fig.1 left). The second block had $12 \times 12$ pixels of $2 \times 2 \mathrm{~mm}^{2}$ size and $2.08 \mathrm{~mm}$ pitch (Fig. 1 center) and the third array had $17 \times 17$ pixels of $1.5 \times 1.5 \mathrm{~mm}^{2}$ and $1.58 \mathrm{~mm}$ pitch (Fig. 1 right). Additionally, for 2 and $1.5 \mathrm{~mm}$ pixel sizes, entrance and lateral faces for all crystal pixels were covered with ESR of 65 microns.

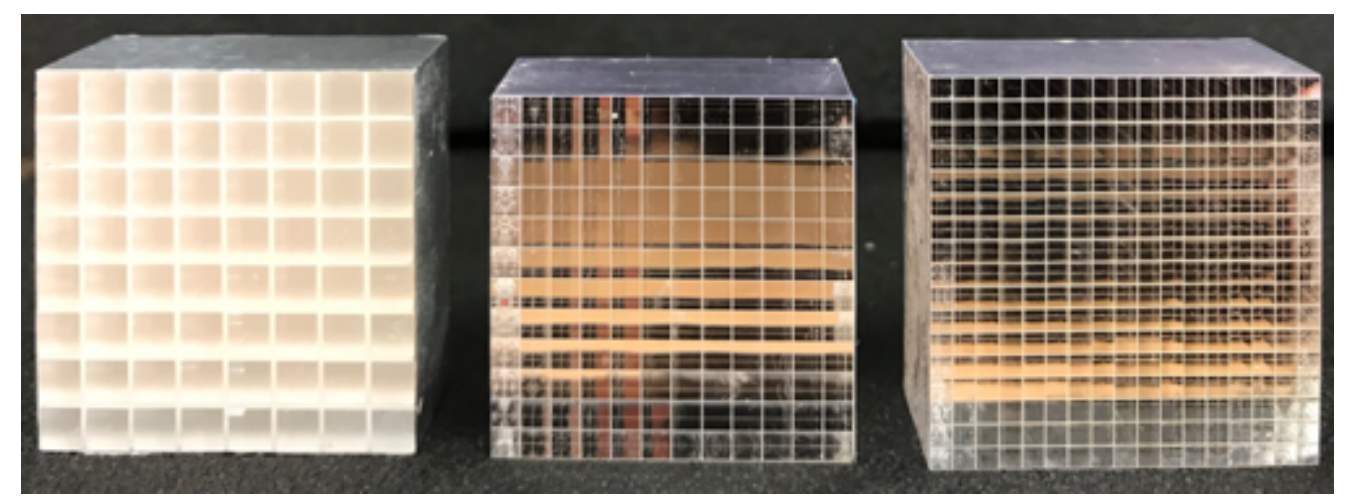

Fig. 1. Left, crystal array with pixel size $3 \times 3 \mathrm{~mm}^{2}$. Center, crystal array with pixel size $2 \times 2 \mathrm{~mm}^{2}$. Right, crystal array with pixel size of $1.5 \times 1.5 \mathrm{~mm}^{2}$. 


\subsection{ASIC readout \& SiPM photosensors}

The crystal arrays were optically coupled using optical grease to SiPM arrays. In particular, we used two arrays of $8 \times 8$ elements, $3 \times 3 \mathrm{~mm}^{2}$ active area, and $3.36 \mathrm{~mm}$ pitch of the type PA3325 from KETEK (Munich, Germany). These arrays, were biased at $28.7 \mathrm{~V} \mathrm{(4} \mathrm{V} \mathrm{over} \mathrm{the} \mathrm{breakdown}$ voltage). The frontend electronics selected to read out all SiPM signals were based on the commercially available Application Specific Integrated Circuit (ASIC) named TOFPET2 from PETsys (Lisbon, Portugal) [10]. Each ASIC reads out 64 signals and integrates high resolution Time-To-Digital converters (30 ps binning) as well as charge integrators for each channel, permitting in that way a quite accurate decoding of gamma-ray pulses.

\subsection{Detector configurations and set-up}

First, two identical detectors based on crystal arrays of $3 \times 3 \mathrm{~mm}^{2}$ size were tested. Thus, each crystal pixel perfectly matched with the photosensor element, resulting in the so-called one-toone coupling. These detectors were tested in coincidence mode, initially for calibration (see below section 2.4) and then for evaluation purposes. Afterwards, one of these detectors was exchanged for the other configuration types, as it will be described below.

When using crystal arrays with $2 \times 2 \mathrm{~mm}^{2}$ and $1.5 \times 1.5 \mathrm{~mm}^{2}$ pixel sizes, that means smaller crystal pitch than the photosensor active area, this resulted in the previously described light sharing configuration. Optical light guides in between the crystal and the sensor were not utilized, except for an additional measurement with the $1.5 \mathrm{~mm}$ pixels. In particular, a $1 \mathrm{~mm}$ thick polished glass layer was added. More detailed description of the light distribution for all three detector configurations is provided in the results section.

All coincidence measurements were carried having the detectors at a distance of $15 \mathrm{~cm}$, and were long enough, in order to avoid statistical fluctuations, that might affect the accuracy of the extrapolated data. A stable temperature environment was ensured in all experiments at $18{ }^{\circ} \mathrm{C}$ $\left( \pm 1^{\circ} \mathrm{C}\right)$. We used a small size ${ }^{22} \mathrm{Na}$ source with $1 \mathrm{~mm}$ in diameter and an activity of $470 \mathrm{kBq}$, centered in a plastic disk of 1 inch in diameter and $6 \mathrm{~mm}$ height.

\subsection{System calibration}

Since the main aim of the experiments was the evaluation of the timing capabilities of each configuration, the correction of the timing skew is critical, which refers to the variations in the time-paths among channels [8]. In order to correct this effect, the two detectors based on the crystal arrays with $3 \times 3 \mathrm{~mm}^{2}$ pixel size were used in coincidence. Two measurements were carried out, first with the source attached to one detector and then to the other one. In both measurements the source was centered into one pixel, using a mechanical holder. Therefore, each detector was completely irradiated in coincidence mode with only one channel in the opposite detector 
(reference detector). We determined the Gaussian centroids of the time differences among all channel pairs of the two detectors (a total of 64 per detector). These values were stored in lookup-tables. This information is unique for each channel and was used as an offset calibration in all following timestamps recordings, independently of the coupled scintillation block, since photosensor array and corresponding ASIC remain the same.

After the skew correction, a time walk calibration was also investigated [8]. The aim was to improve the uncertainties in the timestamp generation related to the signal level. This correction is of special importance when a significant amount of scintillation light is spread among multiple channels, such as in the case of the 2 and $1.5 \mathrm{~mm}$ pixel sizes, due to their geometrical mismatches with the photosensor array. The acquired data were first energy filtered using a 450-572 keV photopeak energy window. Then, we generated 2D plots for each channel of the time difference of its earliest recorded timestamp with the one recorded by the reference detector, as a function of its collected energy. In these 2D plots, projections to the time difference axis were made in very small energy bands. The profiles were fitted with Gaussian distributions, returning for each selected energy band its centroid (and standard deviation), corresponding to a time offset related to the time walk uncertainty. Thus, a time walk calibration file was obtained for each of the detector configurations.

\subsection{Thresholds scan}

Different thresholds can be configured in the TOFPET2 ASIC which impact the timing performance. We carried out an optimization of the so-called vth_t1 threshold, which is directly related to the timestamp generation of each pulse. This task was carried out for all configurations, after applying the time skew correction in the recorded data.

\subsection{Timing, position and energy estimation. Timestamp averaging method}

As it was mentioned above, in addition to the Coincidence Time Resolution (CTR), an evaluation of the spatial and energy resolution of each detector was performed. All results, timing and position related, were obtained after applying a 450-572 $\mathrm{keV}$ photopeak energy window. Energy resolution was calculated, after linearity correction, as the ratio of the FWHM to the distribution centroid. Regarding the position estimation, it was obtained by applying the CenterOf-Gravity (COG) algorithm:

$$
x=\frac{\sum_{i=0}^{i=n} x i \cdot E i}{E i}, \quad y=\frac{\sum_{i=0}^{i=n} y i \cdot E i}{E i},
$$

where xi and yi are the photosensor positions and Ei the collected charge. The total energy was obtained as the sum of channels with an Ei value over the threshold. Regarding the determination of the timing information, when multiple channels (timestamps) are involved, it has been suggested to use the average of timestamps and in particular of weighted averaging 
methods, instead of assigning the timing to the earliest recorded one [8][11]. Herein, for the configuration using the 2 and $1.5 \mathrm{~mm}$ pixels arrays (sections 3.4, 3.5 and 3.6), an energy weighted averaging method using the first $\mathrm{n}$ channel timestamps (Ti) was also used to assign the timestamp to each event:

$$
t_{e v}=\frac{\sum_{i=0}^{i=n} T i \cdot E i}{E i}
$$

\section{Results}

\subsection{Time skew correction}

Fig. 2 shows the time differences among all 64 channels of 1 ASIC, related to the reference one, before and after calibration of the skew time error, respectively. As it can be observed, significant variations in the Gaussian centroids were found, with the deviations inside one single ASIC as large as $1.6 \mathrm{~ns}$.
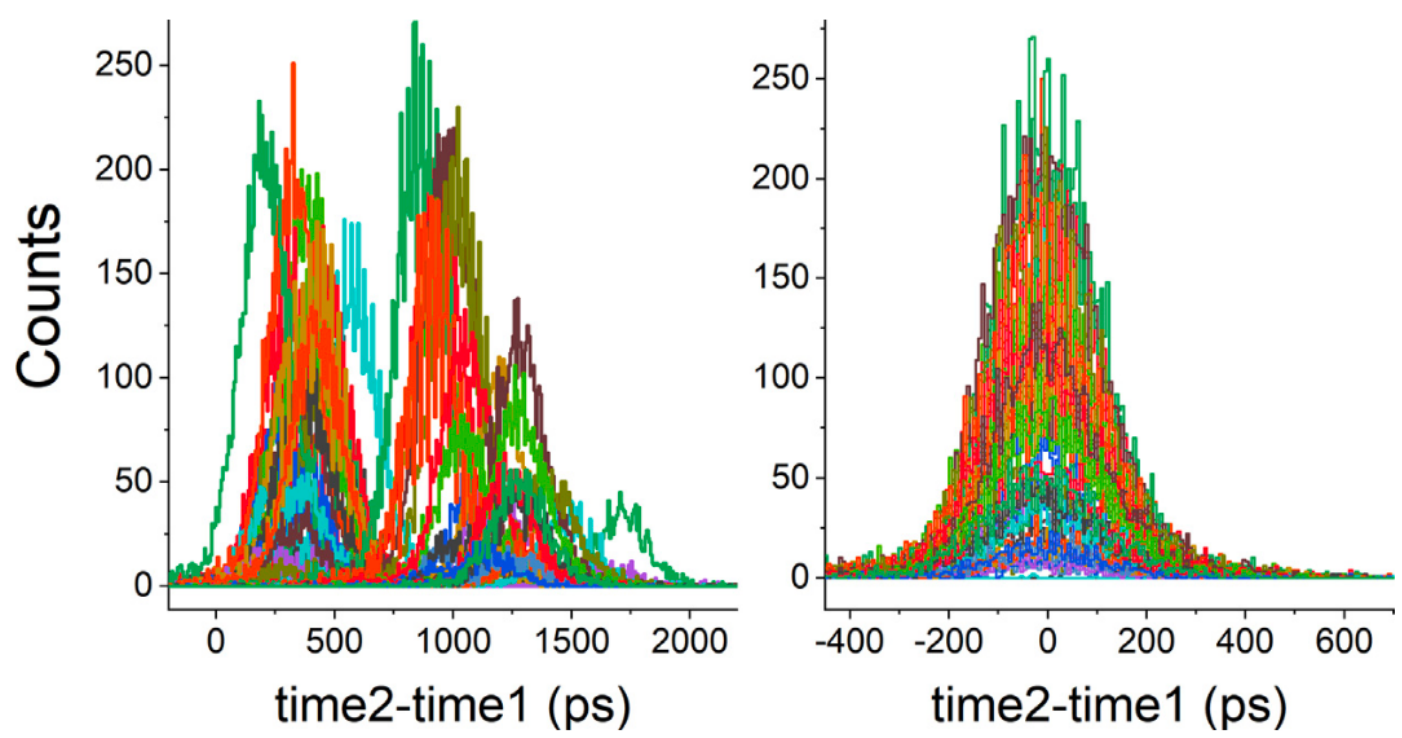

Fig. 2. Left, measured Gaussian centroids of the timing distributions among all 64 ASIC channel pairs (represented by different colors), related to one single channel of the opposite detector (reference detector). Right, time skew correction shifting all Gaussian centroids to 0 .

\subsection{Threshold scan}

After the time skew correction, we obtained the average (whole detector) CTR values as a function of the threshold value for the four tested configurations namely crystal pixels of $3 \mathrm{~mm}$ section, $2 \mathrm{~mm}, 1.5 \mathrm{~mm}$ and $1.5 \mathrm{~mm}$ with the optical window. Fig. 3 depicts the results for the four cases. For the case using $3 \mathrm{~mm}$ pixel size, we did not observe any significant threshold dependency across vth_t1 values. Best results were obtained when using the value of 15 DAQ 
units, providing an average CTR of $238 \pm 6$ ps FWHM (standard deviation of 11 ps) for impacts across the whole detector.

In the case of the $2 \mathrm{~mm}$ pixels, the best average time resolution for all channels was measured using the time threshold corresponding to 7 DAQ units and was found to be $460 \pm 7$ ps FWHM (standard deviation of $21 \mathrm{ps}$ ), before time walk correction. It is observed that 7 ADC units provides the best CTR, but similar values are obtained for the range of 6 to 9 DAQ units.

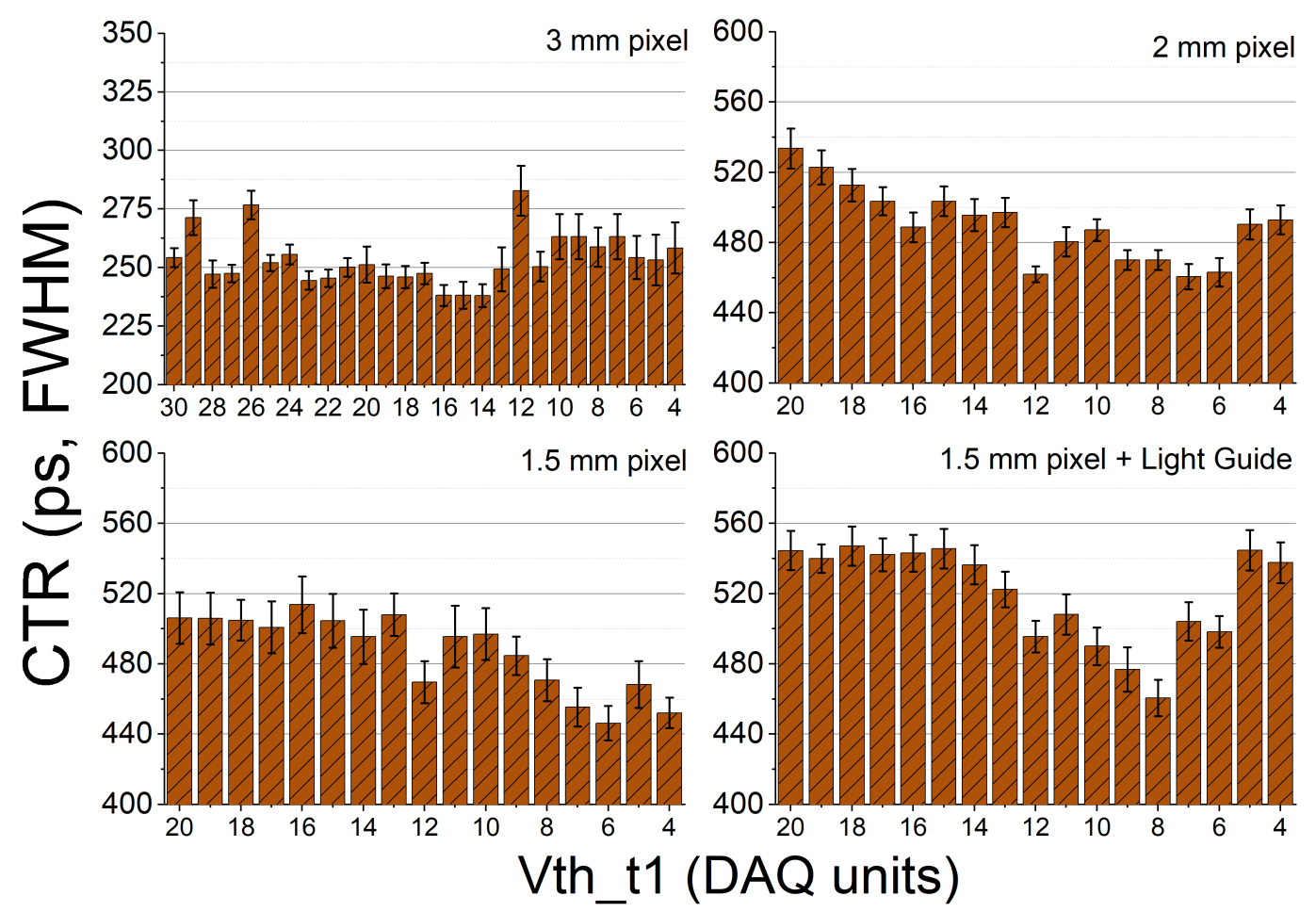

Fig. 3. Average CTR measurements for the whole detector of the four tested configurations, as a function of the threshold level vth_t1.

Similarly to the case of $2 \mathrm{~mm}$ pixels, when testing the $1.5 \mathrm{~mm}$ pixels, best CTR values were found for 6-7 DAQ units (Fig. 3 bottom-left). Considering that the difference in terms of timing resolution between 6 and 7 DAQ units, is inside the estimated error and aiming to avoid even lower threshold that might result to more signal jitter or false triggering, the threshold of 7 DAQ units was selected to be used in all following measurements. For this threshold configuration, the average time resolution before time walk correction, was measured to be $455 \pm 11$ ps FWHM (standard deviation of $22 \mathrm{ps}$ ). When measuring with the $1.5 \mathrm{~mm}$ pixels adding the optical light guide, the average CTR exhibited a similar tendency. The best CTR value was now found to be $460 \pm 10$ ps FWHM (standard deviation of 28 ps) at a threshold of 8 DAQ units. For this configuration, again we observed a worsening of the CTR for very low thresholds. 


\subsection{Analysis for crystals with $3 \times 3 \mathrm{~mm}^{2}$ pixels}

In the detectors based on the one-to-one coupling approach, we observed that on average, $80 \%$ of the generated optical photons for each gamma-ray impact are collected by a single photosensor element, resulting in a sharp rise time of the signal. This suggests to only use the information of one channel for the timing and energy determination.
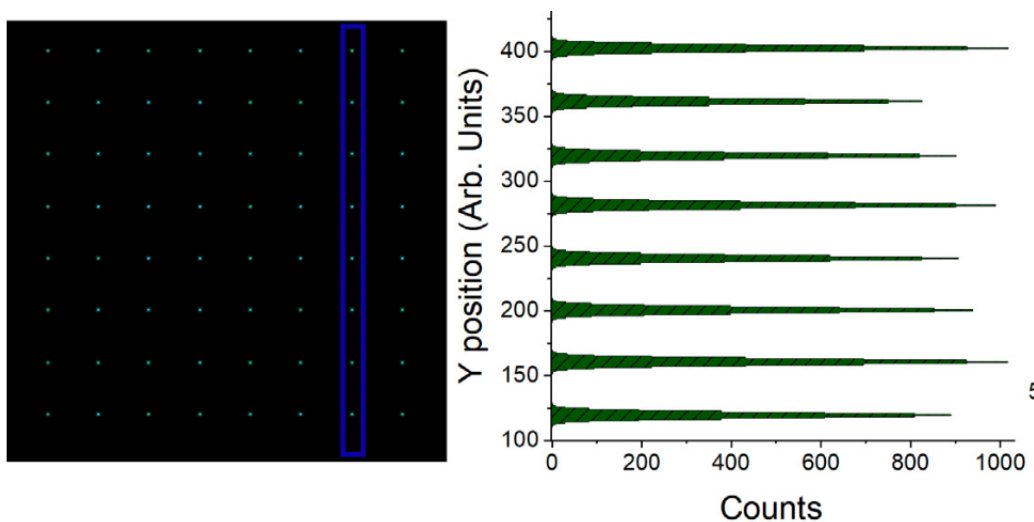

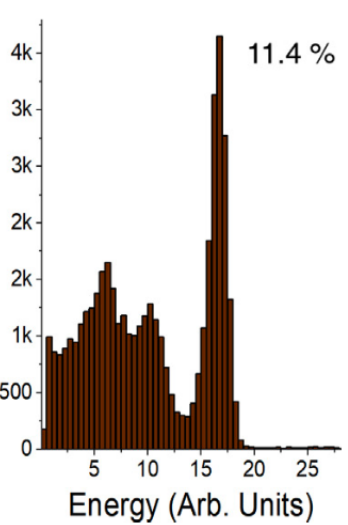

Fig. 4. Left, flood map obtained with the crystal array composed by the $3 \mathrm{~mm}$ crystal pixel. Center, projection data of one column of the crystal array. Right, energy spectra for a central pixel.

As depicted in Fig. 4 left, 2D flood maps of the crystal array were generated aiming to study the spatial resolution as well as the CTR for several Regions of Interests (ROIs). Regarding the spatial resolution, all pixels are well resolved, providing a detector spatial resolution of $3 \mathrm{~mm}$. We also analyzed the peak-to-valley ratio obtaining $936 \pm 68$ and on average one count per bin between peaks.

ROIs for each crystal pixel in one column were made to study the energy and timing resolution as a function of the pixel position. Regarding the energy resolution, we found an average energy resolution for all pixels in that column of $11.4 \%$ with a standard deviation of $0.7 \%$, after correction for the SiPM saturation. Fig. 4 right shows the energy spectra of one pixel belonging to this column.

Fig. 5 shows the measured CTR as a function of the pixel position in the same selected column after applying the energy window filter. The sketch on the top depicts the relative crystal pixel and SiPM positions. We found a homogeneous CTR across all pixels, with an average of 242 ps (standard deviation of 6 ps), and best value the $232 \pm 4$ ps FWHM. From the average value of 242 ps FWHM we have determined a Detector Time Resolution (DTR) of 171 ps FWHM for this detector configuration. 


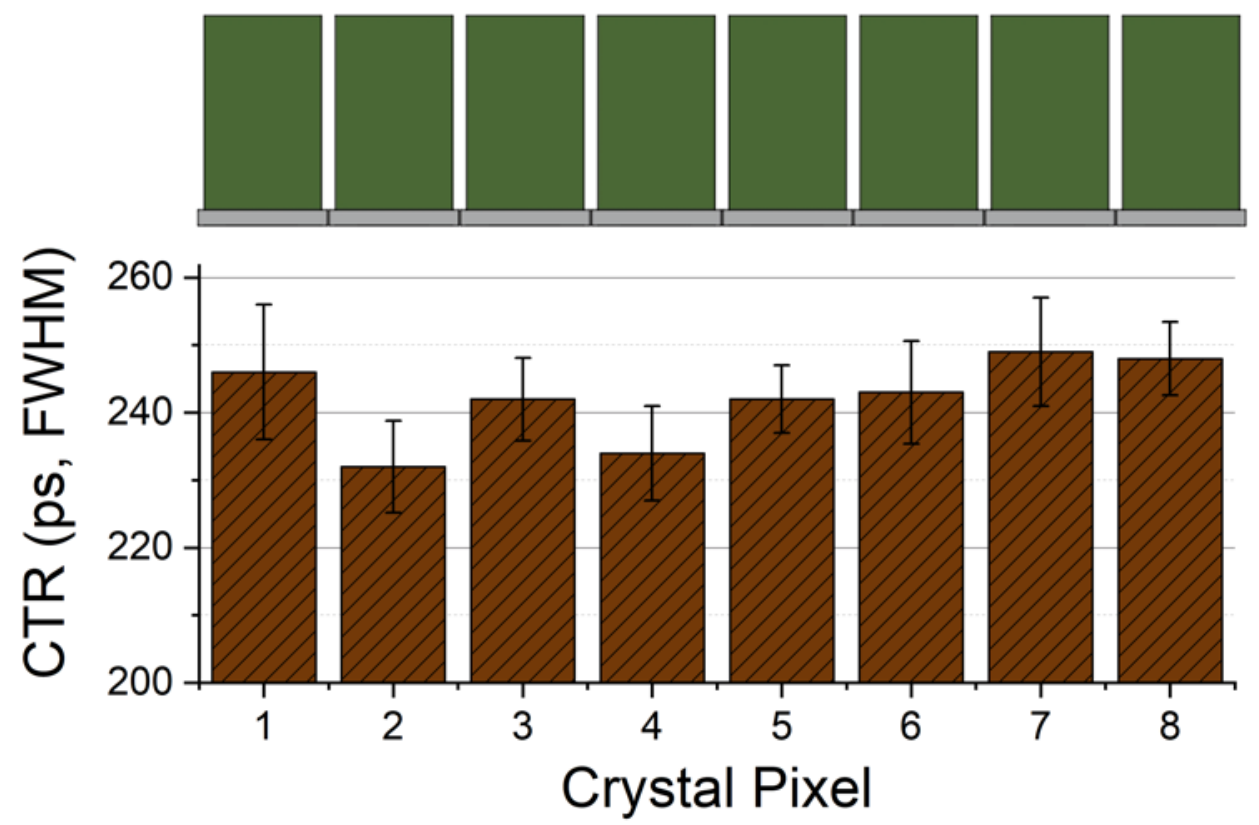

Fig. 5. Top, relative position of each crystal pixel belonging to one column, with respect to the photosensor elements. Bottom, CTR measured when filtering each crystal pixel individually.

\subsection{Analysis for crystals with $2 \times 2 \mathrm{~mm}^{2}$ pixels}

When using the crystal arrays of $2 \mathrm{~mm}$ pixels coupled to $3 \mathrm{~mm} \mathrm{SiPM}$ (in coincidence with the reference detector of $3 \mathrm{~mm}$ pixels) due to the geometrical mismatch between them, light sharing was expected leading to the triggering of several SiPM (ASIC channels) for each gammaray event. During the data analysis, we measured an average of $40 \%$ of the scintillation light captured by only one SiPM while the rest was shared to surrounding photosensors. This generates a variability of signal amplitudes in multiple readout channels for each event. Moreover, lower signals are typically affected by noise during the generation of the timestamp due to the time walk error.

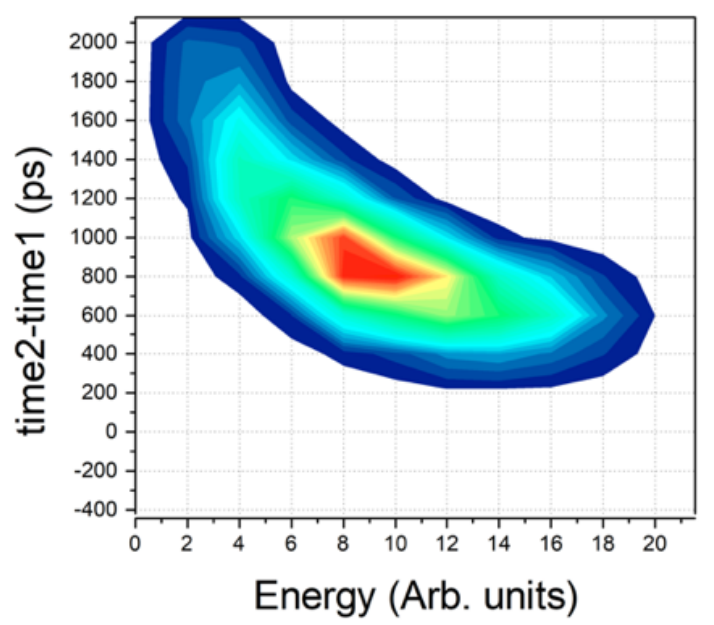

Fig. 6. Contour plot of the time differences of one pair of channels as a function of the energy of the earliest hit showing the time walk influence. 
Figure 6, depicts the time differences of the earliest recorded timestamp with the one recorded by the reference detector, as a function of the collected charge in DAQ units for a given pair channel. As it was also described above, a calibration method was carried out to compensate the delay in the timestamp generation as a function of the signal amplitude. In a following measurement using this configuration, after correction of the timestamps for the time walk, an average timing resolution of $381 \pm 6$ ps FWHM was reached (for the whole detector).
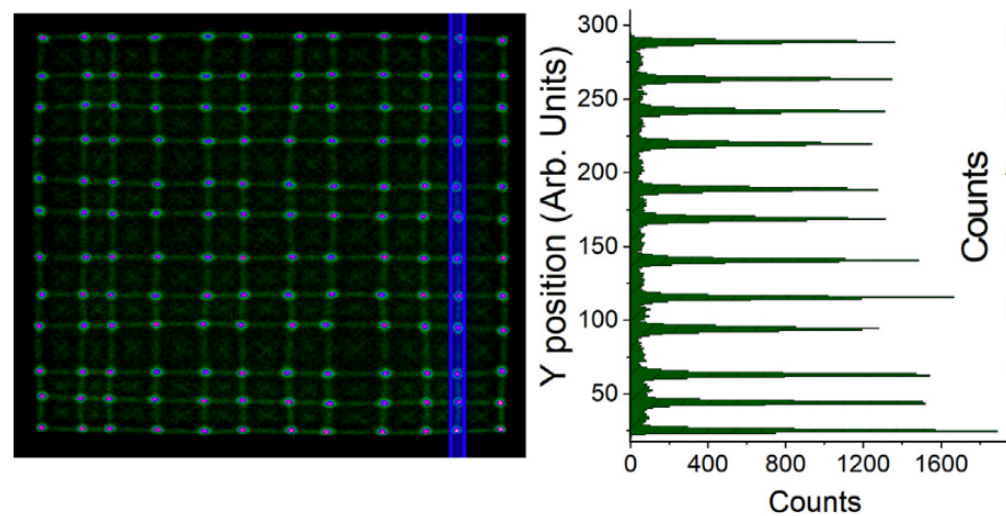

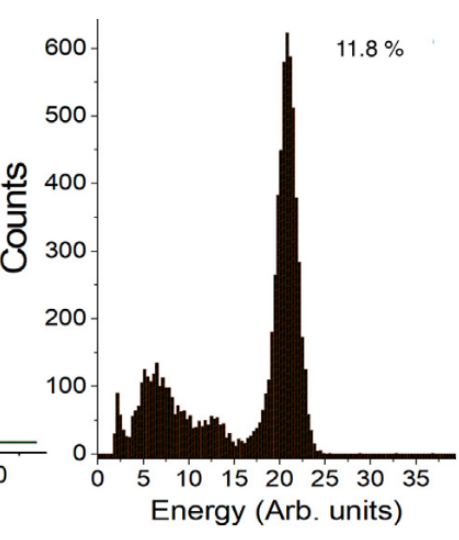

Fig. 7. Left, flood map obtained with the crystal array of $2 \mathrm{~mm}$ pixels. Center, projection of one column of the crystal array. Right, energy profile for the one pixel belonging to the filtered ROI.

Fig. 7 left exhibits all $12 \times 12$ crystal elements clearly resolved with a peak-to-valley ratio $46 \pm 7$. The average energy resolution for the 12 crystal elements belonging to the filtered column of crystals was found to be $11.8 \%$ with a standard deviation of $0.5 \%$. In this case the time analysis, besides including the CTR obtained when using the earliest timestamp, we also include the results obtained when using a weighted by energy averaging method.

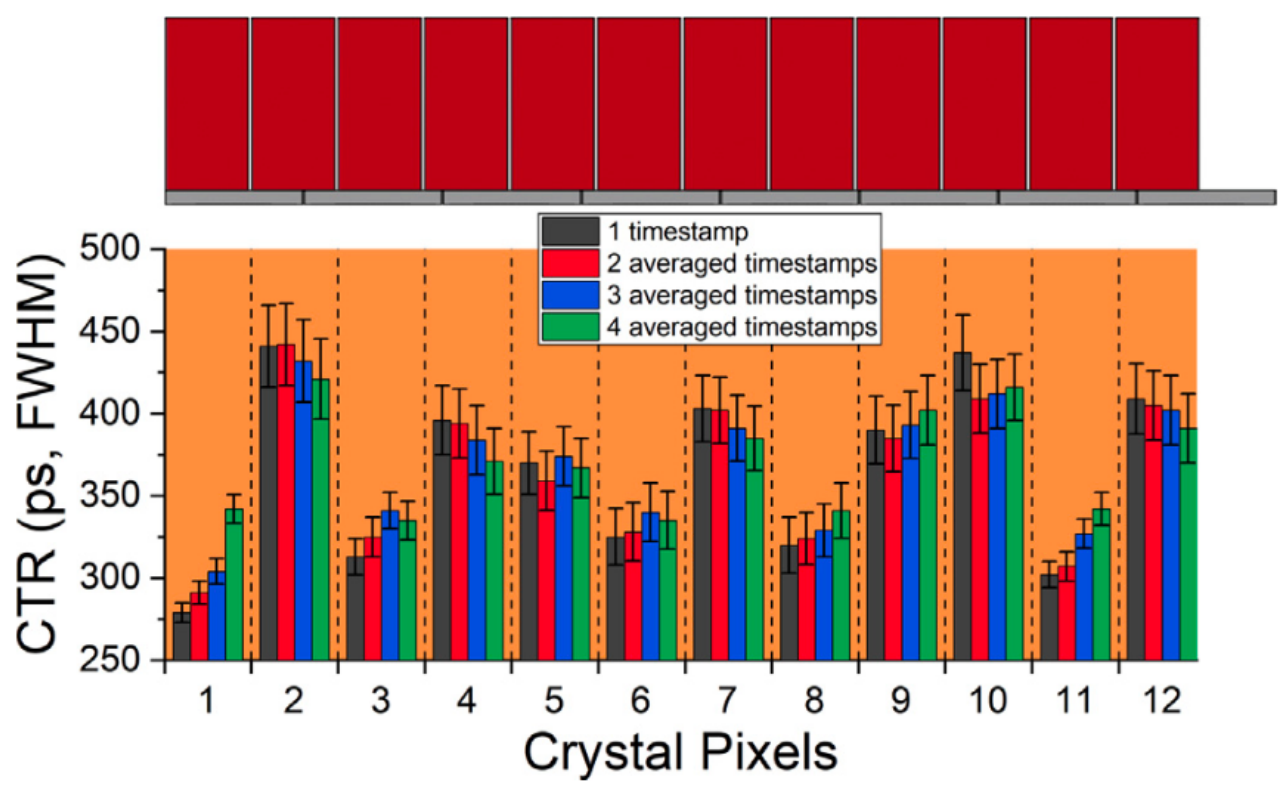

Fig. 8. Top, relative position of each crystal pixel belonging to one column, with respect to the photosensor elements. Bottom, CTR for each crystal pixel, obtained using the earliest timestamp and an average energy weighting timestamp method after applying a position (ROI) and energy filter. 
As it can be appreciated in Fig. 8, when using one timestamp, the measured CTR for this group of pixels, varies from $279 \pm 6$ ps to $441 \pm 25$ ps FWHM with an average value of 365 ps FWHM (standard deviation of $55 \mathrm{ps}$ ). These variations among pixels, are related to each pixel position and, thus, the light collection efficiency. The weighted averaging method, although did not provide better average values compared to the use of 1 timestamp, it reduces the standard deviation among pixels, being $32 \mathrm{ps}$ for the case of 4 weighted timestamps (average value $370 \mathrm{ps}$ FWHM).

\subsection{Analysis for crystals with $1.5 \times 1.5 \mathrm{~mm}^{2}$ pixels}

The crystal array with $1.5 \mathrm{~mm}$ pixels was coupled to the photosensor array without any optical guide in between, despite the small crystal pixel size compared to the photosensor pitch (3.36 $\mathrm{mm})$. As depicted in Fig. 9 left, all $17 \times 17$ crystal elements were resolved. After again applying the ROI filtering for a column of pixels, a peak-to-valley of $7 \pm 1$ was measured. However, some deterioration is observed at the detector block edges due to a poorer light collection. The average energy resolution for the filtered column was found to be $13 \%$ with a standard deviation of $1 \%$ (see Fig. 9 right).
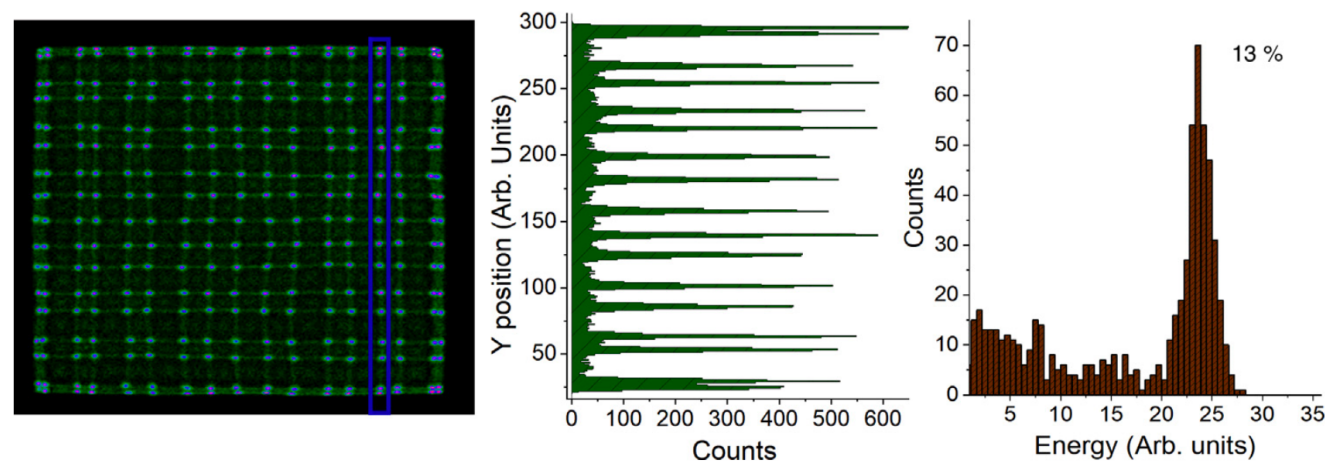

Fig. 9. Left, flood map obtained with the crystal array composed by the $1.5 \mathrm{~mm}$ crystal pixel. Center, projection of one column of the crystal array. Right, energy profile for one pixel belonging to the filtered column.

The time walk correction was also applied to these data, following the methodology described above. In a measurement using this configuration, the average time resolution for the whole detector block after the time walk correction was found to be $365 \pm 4$ ps FWHM. A more detailed time analysis for the 17 crystal pixels contained in one column of the crystal array was carried out, again using the earliest timestamp as well as the energy weighted averaging method. As it can be seen in Fig. 10, when using one timestamp values as good as $241 \pm 5$ ps FWHM were achieved for crystals whose exit face was fully contained to just one photosensor element. In contrast to this, pixels which were coupled in between several photosensor elements, showed worse time resolution reaching $370 \pm 23$ ps FWHM. Eventually, an average CTR of 303 ps FWHM (standard deviation of $37 \mathrm{ps}$ ) could be obtained for this detector configuration and this column of pixels. In the case of the weighted averaging method, best results were found when 3 timestamps are averaged. Although the average CTR among pixel remained 303 ps FWHM (as 
in the case of the earliest timestamp) the standard deviation in this case slightly improved to 26 ps.

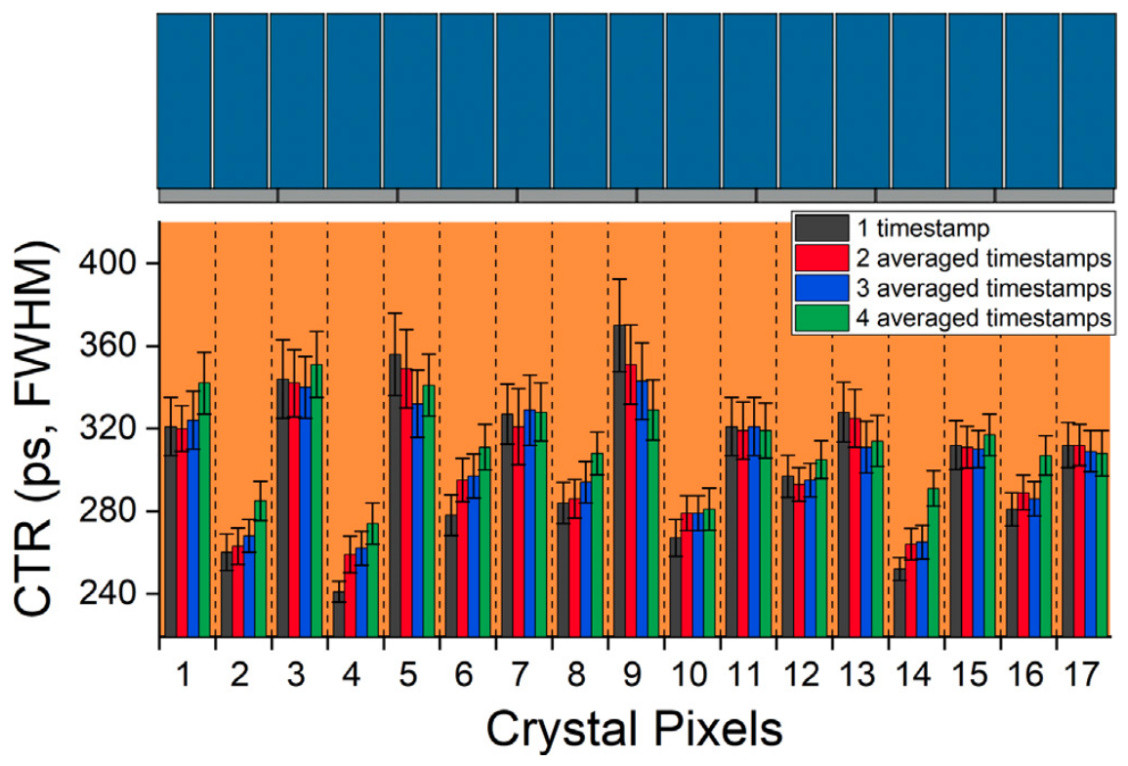

Fig. 10. Top, relative position of each crystal pixel belonging to one column, with respect to the photosensor elements. Bottom, CTR for each crystal pixel measured with the first timestamp recorded as well as with weighted averaging method, after applying an energy and position (ROI) filter.

\subsection{Analysis for crystals with $1.5 \times 1.5 \mathrm{~mm}^{2}$ pixels and optical light guide}

The crystal array with $1.5 \mathrm{~mm}$ pixels was also tested using a glass layer of $1 \mathrm{~mm}$ thick in between the scintillator and photosensor array (coupled using optical grease). The aim was to improve the spatial resolution near the detector block edges. The use of an optical guide allows one for a better pixel identification, as it generates a wider scintillation light spread. As it can be seen in Fig. 11 left, all pixels are again resolved, including the crystal edges. Moreover, there is a better homogeneity in the pixel identification, in contrast to the case without the glass window where pixels tend to concentrate within the SiPM center. Using this configuration, we determined an average peak-to-valley ratio of $9.5 \pm 1.6$. An average energy resolution measured for each of the studied 17 crystal elements contained in one column, was found to be $12.5 \%$ with a standard deviation of $0.7 \%$.

Regarding the timing resolution, the use of the optical window might affect the single channel SNR and, consequently the CTR. In a following measurement, after correcting for the time walk, the average detector timing resolution was found to be $428 \pm 7$ ps FWHM. Then, the CTR was determined after applying the positioning (ROI) filter for all 17 pixels contained in one column. The first timestamp recorded and a weighted by energy averaging method was again used, expecting some improvement compared to the only one timestamp due to the more intense light sharing. As the analysis revealed, when using four timestamps, better timing performance was found. In particular for the cases of 1, 2, 3, 4 and 5 timestamps, time resolutions of $418 \pm 43$, 
$376 \pm 26,358 \pm 23,360 \pm 20$ and $367 \pm 16$ ps were obtained, respectively. That being said, averaging weighted timestamps, contributed to the improvement of both of the average value of pixels as well as in the deviation among them. Fig. 12 shows the CTR as a function of the pixel position and for different number of averaged weighted timestamps.
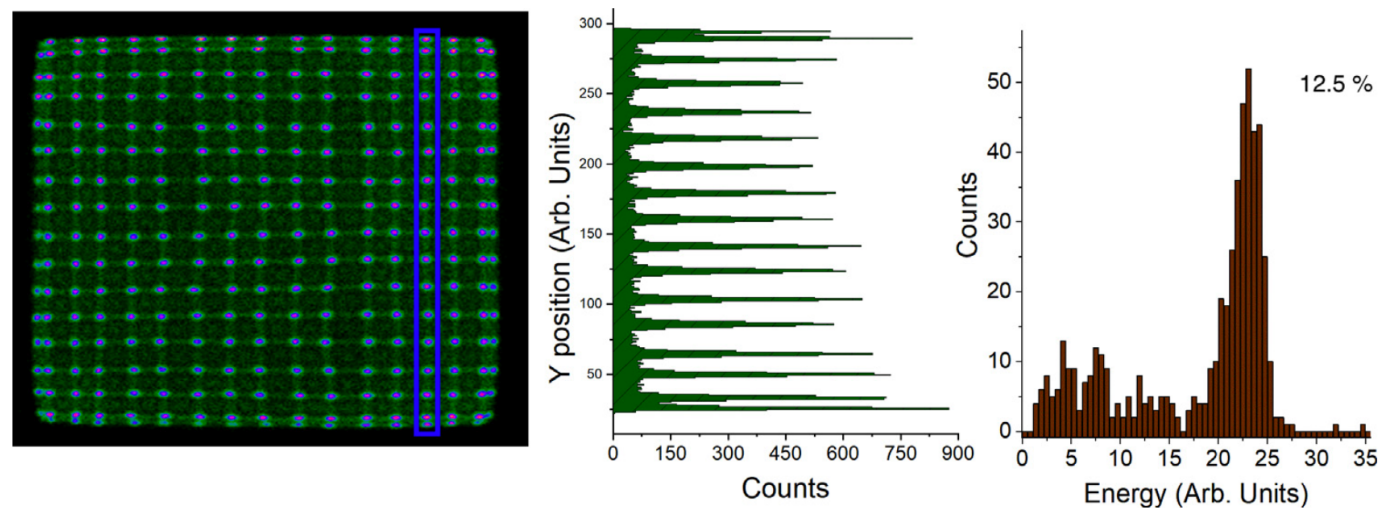

Fig. 11. Left, flood map obtained with the crystal array composed by the $1.5 \mathrm{~mm}$ crystal pixel and optical light guide. Center, projection of one column of the crystal array. Right, energy profile for a pixel of the filtered column (energy filtered).
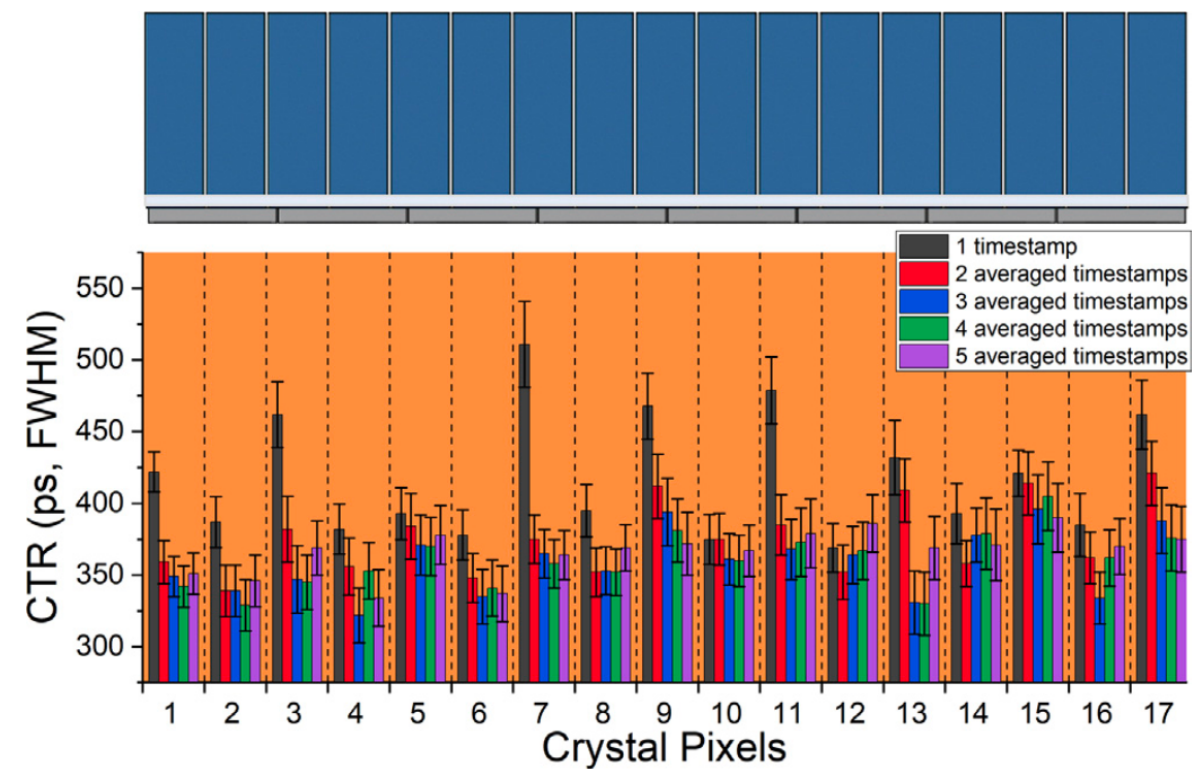

Fig. 12. Top, relative position of each crystal pixel belonging to one column, with respect to the SiPMs. Bottom, time resolution measured for each crystal pixel (after applying the energy and ROI filter), using the first corrected timestamp and an energy weighted averaging method.

\section{Discussion}

In terms of spatial resolution, all tested crystal pixel sizes ( $3 \mathrm{~mm}, 2 \mathrm{~mm}$, and $1.5 \mathrm{~mm}$ ) were resolved when coupled to $3 \times 3 \mathrm{~mm}^{2}$ photosensor arrays. Additionally, we observed that small crystals of $1.5 \mathrm{~mm}$ were distinguishable including those at the edges, although the last ones and in particular the pixels in the corners showed a worst peak-to-valley ratio due to the lower amount of photosensors involved in the scintillation light collection process. Significant improvement 
however, was observed in these regions when using the glass window. Generally, high values of the peak-to-valley ratios were found for the case of using $3 \mathrm{~mm}$ and $2 \mathrm{~mm}$ pixel, whereas lower ones were found for the rest of configurations. It is worth mentioning that in the flood map obtained with the $3 \mathrm{~mm}$ crystal size, hardly a background can be seen. This behavior, is expected to be a result of a combination of two factors. Firstly, the lower probability for inter-crystalscattering compared to the cases of smaller crystal elements $(1.5$ and $2 \mathrm{~mm})$, and secondly, the fact that a relatively high threshold was used in this configuration, resulting more improbable that the surrounding sensors collect enough photons for triggering the electronics and have an effect in the COG algorithm. Energy resolution was also evaluated during the data analysis for a group of pixels belonging in one column and, for all configurations. In general, the energy resolution of individual pixels was measured to be in the range of 11-13\% (after energy calibration).

Both average detector as well as individual crystal pixels CTR were determined for all cases. We determined first the optimum ASIC $v t h \_t 1$ threshold value for each case. When using $3 \mathrm{~mm}$ pixels the, dependency of the time resolution as a function of the threshold, was not found to be significant. However, best values were determined for the range between 14-16 DAQ units. All other cases seem to exhibit best values around 6-8 DAQ units. For the case of using the glass window, worst values were found for lowest DAQ values, most likely due to a poorer SNR of individual channels.

The best CTR of 232 ps was obtained with the configuration based on the one-to-one coupling approach ( $3 \mathrm{~mm}$ pixels). In the case of smaller crystal elements, scintillation light eventually spreads among few SiPMs. Indeed, we observed the CTR dependency as a function of the crystal pixel position and SiPM element. The use of an energy weighted averaging method for the timestamp determination, although it did deteriorate the average timing resolution in most of the cases, it did permit the reduction of the standard deviation among individual pixel capabilities. This approach, can be of high importance during a reconstruction process. However, as was shown above, in the case of $1.5 \mathrm{~mm}$ pixel and the lightguide, significant improvement was observed when 3 or 4 timestamps were used.

\section{Conclusions}

We have carried out a complete performance evaluation of four gamma-ray detector configurations, especially suitable for PET. Spatial, energy and timing resolution were studied in detail. The detector designs could be employed for clinical ( $3 \mathrm{~mm}$ pixels) but also for pre-clinical $(1.5 \mathrm{~mm})$ applications, with accurate timing capabilities.

Results showed the one-to-one coupling approach providing the best results in terms of timing and energy resolution due to, on average, a higher collection of optical photons by a single channel. Using this set-up, we have been able to reach a CTR as good as 232 ps FWHM. We are aware that alternative photosensors with improved timing capabilities using wider microcell sizes 
could improve these results by about 15\% [12][13]. Also, the use of Ca-doped LYSO crystals could also improve the CTR by 10-20 ps FWHM [14].

When crystal pixels smaller than SiPMs active area are tested, in addition to the skew time correction, a time walk correction and, eventually, in one case the weighted averaging of several timestamps was required to provide a more accurate CTR. For the case of $2 \mathrm{~mm}$ crystal pixels, a CTR of 365 ps FWHM was measured for a column containing 12 crystal elements. When applying ROI filters in the crystal pixels contained in this column, we measured values equal to 279 ps. The crystal array based on the $1.5 \mathrm{~mm}$ pixel size, was tested with and without the use of an optical glass window of $1.0 \mathrm{~mm}$ thickness. As expected, the pixel identification was more homogeneous when using a glass window, including the edges, but at the cost of some CTR deterioration. In particular, for one group of pixels, an average value of 303 ps FWHM, including time walk correction, degraded to 418 ps when the light guide was used. However, this last value improves to 358 ps FWHM when the energy averaged timestamp method was used. When analyzing the CTR for a given pixel, a 241 ps FWHM was achieved without the light guide, worsening to 322 ps with the glass layer including the averaging of 3 weighted timestamps.

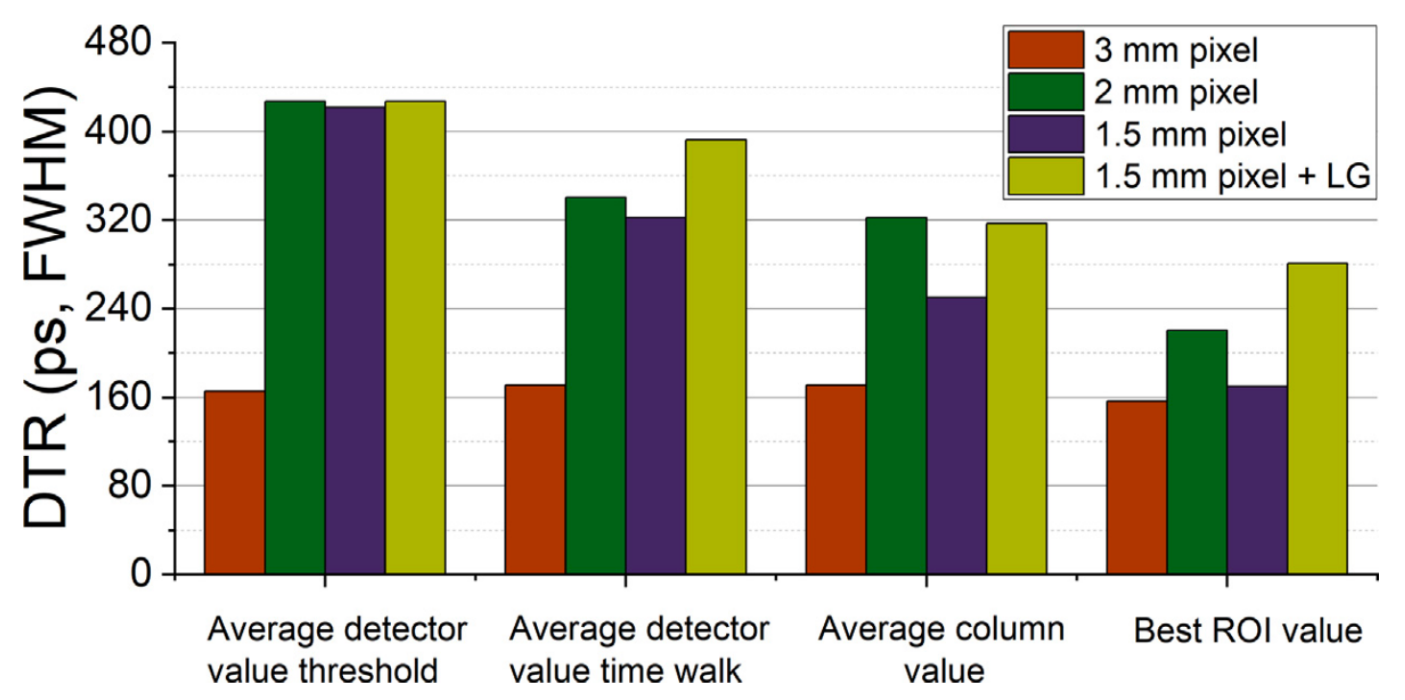

Fig. 13. Summary of measured DTR for the four tested configurations, following the optimization in terms of threshold, time walk correction and after applying positioning filters

Since the same reference detector was used in all experiments, and its contribution was determined by the experiments described in section 3.3, the DTR could be estimated for each case studied. In particular, the DTR for the configuration with one-to-one coupling was 171 ps FWHM. We have obtained the DTR for all configurations and processes described in this work, as shown in Fig. 13.

Summarizing, it is possible to reach good detector performance in terms of pixel identification and energy resolution, when using $3 \mathrm{~mm}$ photosensor arrays. The results exhibited in this work regarding spatial resolution, suggest that crystal pixels smaller than $1.5 \mathrm{~mm}$ could also be 
resolved. The time resolution however, strongly depends on the geometrical position of each crystal pixel with respect to each photosensor element. Nevertheless, even in the detector configurations in which a significant mismatch between crystal and SiPMs existed, values of CTR as low as 241 ps FWHM were achieved. Some of the proposed designs might be well suitable for the development of clinical and pre-clinical TOF-PET detectors and provide solutions for detector concepts which combine good spatial and timing resolutions.

\section{Acknowledgments}

This project has received funding from the European Research Council (ERC) under the European Union's Horizon 2020 research and innovation program (grant agreement No 695536) and by the Spanish Ministerio de Economía, Industria y Competitividad under Grant TEC201679884-C2-1-R. The first author has also been supported by Generalitat Valenciana under grant agreement GRISOLIAP-2018-026.

\section{References}

[1] T. Jones, History and future technical innovation in positron emission tomography. J. Med. Imag. 4:1 (2017), 11013.

[2] S. Surti, Update on time-of-flight PET imaging. J Nucl Med. 56:1 (2015), 98-105.

[3] P. Lecoq, Pushing the Limits in Time-of-Flight PET Imaging. IEEE Transactions on Radiation and Plasma Medical Sciences, 1:6 (2017), 473-485.

[4] S. Surti and J. S. Karp, Advances in time-of-flight PET. Phys Med. 32:1 (2016), 12-22.

[5] S. Gundacker et al., Measurement of intrinsic rise times for various L(Y)SO and LuAG scintillators with a general study of prompt 728 photons to achieve 10 ps. Phys Med Biol. 61:7 (2016), 2802-37.

[6] A. González-Montoro et al., Novel method to measure the intrinsic spatial resolution in PET detectors based on monolithic crystals. Nucl Instr Methods. 920 (2019), 58-67.

[7] W. W. Moses, Fundamental Limits of Spatial Resolution in PET. Nucl Instrum Methods Phys Res A. 648:1 (2011), S236-S240.

[8] E. Lamprou et al., Exploring TOF capabilities of PET detector blocks based on large monolithic crystals and analog SiPMs. Physica Medica. 70 (2020), 10-18.

[9] E. Lamprou et al., PET detector block with accurate 4D capabilities. Nucl Instr Methods. 912 (2018), 132-136.

[10] A. Di Francesco et al. TOFPET2: A high-performance ASIC for time and amplitude measurements of SiPM signals in time-of-flight applications. Journal of Instrumentation. 11:3 (2016), C03042. 
[11] T. H van Dam et al., Sub-200 ps CRT in monolithic scintillator PET detectors using digital SiPM arrays and maximum likelihood interaction time estimation. Phys Med Biol. 58:10 (2013), 3243-57.

[12] V. Nadig et al., Evaluation of The PETsys TOFPET2 ASIC in multi-channel Coincidence Experiments arXiv:1911.08156.

[13] S. Gundacker et al., High-frequency SiPM readout advances measured coincidence time resolution limits in TOF-PET. Phys Med Biol. 64:5 (2019).

[14] S. Gundacker et al., State of the art timing in TOF-PET detectors with LuAG, GAGG and L(Y)SO scintillators of various sizes coupled to FBK-SiPMs. JINST. 11 (2016), P08008. 


\section{Discussion}

In the literature, several works have already been published, in which monolithic crystals are combined with digital SiPMs photosensors reporting impressive timing results reaching about 200 ps FWHM. However, dSiPMs are still somehow a special photosensor case, only commercialized by one company, somehow limiting a broader use. Moreover, they account for inherent disadvantages such as massive requirements of readout electronics or low temperature cooling. Therefore, we found interesting and potentially revolutionary a more extended approach based on analog photosensors (SiPM). Those devices exhibit a lower cost and higher accessibility. Through a number of works presented in this doctoral thesis, we observed the intense light sharing to many photosensor elements that occurs when making use of a monolithic scintillation block. This fact, challenges the extrapolation of a good timing resolution. Notice that digital SiPMs allow one to define very low threshold levels but at the cost of disabling noisy cells and requiring lower operating temperatures. However, as we demonstrated, there are still ways to enhance the timing performance when using monolithic blocks, analog SiPMs and ASICs. A series of corrections namely time-skew and time-walk calibrations, specifically developed under the framework of this thesis, as well as post-processing methods such as false noise-related events rejection and timestamps averaging methods can notoriously improve the timing determination of the gamma-ray impact. After applying those methods, we observed important improvements in the detector time resolution. We worked with large LYSO scintillators of $50 \times 50 \times 15 \mathrm{~mm}^{3}$, following the original aim to be suggested for both clinical and pre-clinical scenarios. We found average DTR of the non-processes data (RAW) over $1 \mathrm{~ns}$ FWHM, decreasing to values nearing 497 ps FWHM for impacts along the whole crystal when these methodologies were applied. Those values were further improved down to 371 ps FWHM for specific VOIs in the crystal (section 4.3). Additionally, we do expect that these numbers can further be improved if different photosensor types are used and combined with other crystal geometries and/or treatments. In pilot tests not shown in this $\mathrm{PhD}$ work we have determined DTR values below 300 ps FWHM. Definitely, in this configuration, timing results are not yet in the desired range neither comparable to the state-of-the-art values obtained with crystal arrays, but we are confident we will be approaching these numbers after further understanding the light spread behavior and timing determination of poor SNR signals.

We also evaluated and compared crystal arrays with varying size dimensions under the same measuring conditions. In detail and as described in section 4.4, pixels sizes of 1.5, 2 and $3 \mathrm{~mm}$ and $10 \mathrm{~mm}$ height were tested. Very interestingly and not studied before in detail to our knowledge, we were able to obtain a deep understanding as well as to demonstrate how the crystal pixel position relative to the photosensor element position, affects the timing resolution. For instance, we observed how individual pixels centered to one photosensor show significantly better time resolution than others located in the junction of multiple photosensors. It is worth mentioning 
the studied case of $1.5 \times 1.5 \mathrm{~mm}^{2}$ crystal elements in which we determined a CTR differences ranging from 241 to 370 ps FWHM, provoked by this crystal-SiPM relative geometrical position. This study strongly demonstrates the importance of a high light collection by a given photosensor element. In the configurations shown in this work both when using monolithic crystals and crystal arrays with sizes smaller than the photosensor active we seek to combine high spatial impact determination and timing capabilities. Unfortunately, these cases result in the scintillation light sharing among several photosensor and, thus, a reduced SNR. 


\section{Conclusions and future work}

In the field of medicine, there is a recognized value of ensuring an accurate disease or lesion diagnosis, accessible to everyone. Among the great number of disciplines related with this field, in the present doctorate assertion we focused on proposing and evaluating new concepts of PET detectors, which can significantly increase the value of molecular imaging both in clinical and pre-clinical practice. The goals of the next generation PET scanners are well defined: timing and spatial resolution should be combined in the most efficient way possible. This will facilitate the precise imaging and quantification of all occurring molecular abnormalities inside the subject under study. Following this direction, we have carried out multiple studies proposing new detector concepts which will infer all desired characteristics and, thus, be candidates for future PET applications and developments.

The work presented in this $\mathrm{PhD}$ thesis through the different papers, shows a deep study and understanding of the timing determination of gamma-ray impacts in detector blocks based on scintillators, analog SiPM and ASIC-based readout electronics. We have put significant emphasis in understanding the processes that occur in the scintillation light production and photosensor collection, as well as in their conversion to measurable signals. ASICs are available since long, but only recently are integrated in the design of PET systems. We exhibited the limits of combining thick monolithic crystals with analog SiPM and ASICs, but also showed ways of improving the RAW results. In the last work we made possible to make use of crystals arraysbased configurations, and achieve high spatial resolution and state-of-the-art timing capabilities.

Following the objectives, the first and main approach of this $\mathrm{PhD}$ work was driven by the ambitious aim to develop a PET detector with accurate 4D capabilities (spatial and timing information) and its implementation in organ-dedicated PET system with limited angular coverage. Our purpose was to combine monolithic scintillators which are well known for their great spatial capabilities $(\mathrm{x}, \mathrm{y}, \mathrm{z})$, with analog SiPM photosensors and, an ASIC readout to also extrapolate good timing performance. While monolithic scintillators have been highly exploited in PET for both clinical and small animal imaging practice, and as shown in this work can permit spatial resolution nearing $1 \mathrm{~mm}$, to our knowledge this is the first time combining this type of scintillators with analog SiPMs and an ASIC-based readout for a detailed evaluation of their timing capabilities in PET applications.

The former results also suggested the investigation of alternative TOF-PET detectors based on crystal arrays, capable of combining both high spatial and temporal capabilities. A detailed study of their overall performance was carried out, also fulfilling the thesis objectives.

Currently, the candidate is still focusing on translating all the acquired multidisciplinary knowledge to novel detector and system configurations. In the short term, he has already included 
this effort in an organ-dedicated TOF-PET prototype. In this line of investigation, he aims to demonstrate in practice how an accurate time resolution can benefit PET imaging, and provide new diagnostic tools. The prototype, that will be presented under the name CardioPET is composed by 3072 detector elements and is optimized for heart imaging under stress condition. This implementation is taking place within the framework of the Spanish grants [GRISOLIAP2018-026] and [TEC2016-79884-C2-1-R]. The state-of-the-art combination of a coincidence time resolution nearing $238 \mathrm{ps}$ with a spatial resolution of a $1.5 \mathrm{~mm}$ ( $3 \mathrm{~mm}$ pixels) makes it a novel and interesting design. In another parallel line of research, he seeks the development of ultrahigh-resolution detectors both spatial and timing, suitable for mouse brain imaging. This work is carried out under an ERC Advanced grant [Grant agreement No. 695536]. In this direction, he demonstrated that a matrix of $3 \times 3$ pixels of $1 \times 1 \times 3 \mathrm{~mm}^{3}$ coupled to SiPM elements of $3 \times 3 \mathrm{~mm}^{2}$ (in $4 \times 4$ elements SiPM array), were easily resolved while they can reach a DTR below 100 ps FWHM. Meanwhile, since the candidate is positive about the significant advantages of the light sharing approach, is still seeking for the integration of monolithic and/or semimonolithic crystals in TOF-PET applications, aiming to improve the standards in molecular imaging.

Reaching at the end of this thesis, we consider that we have created a valuable reference for future efforts related with PET instrumentation. Prior to the works published under the framework of this $\mathrm{PhD}$, limited information could be found in bibliography regarding the TOF potential of detectors based on light sharing configurations, despite the fact that these designs consist a standardized and successful approach in PET. Having now proved that TOF resolution can be achievable also in these types of designs, hopefully, researches and engineers will may found an additional motivation to keep on with this research line, despite the challenges shown up. The great thing is that PET still has great room for improvement, and as long as we combine properly all efforts, we could mark another milestone in PET and eventually in modern medicine. 


\section{Appendix}

In addition to the articles selected to compose this compendium of articles, a series of more have been published under the framework of this PhD thesis. The complete list, including the selected ones, is depicted below.

\section{Peer-reviewed Publications}

1. G. Cañizares, A. Gonzalez-Montoro, M. Freire, E. Lamprou, J. Barrio, F. Sanchez, J.M. Benlloch, L. Hernandez, L. Moliner, L.F. Vidal, I. Torres, P. Sopena, C.D. Vera Donoso, P. Bello, J. Barbera and A.J. Gonzalez, Pilot performance of a dedicated prostate PET suitable for diagnosis and biopsy guidance. EJNMMI Physics, 7:31 (2020), 38.

2. E. Lamprou, F. Sanchez, J.M. Benlloch and A. J. Gonzalez, In-depth evaluation of TOFPET detectors based on crystal arrays and the TOFPET2 ASIC. Nucl. Instrum. Methods Phys. Res. A., 977 (2020), 164295.

3. A. J. Gonzalez, J. Barrio, E. Lamprou, V. Ilisie, F. Sanchez and J. M. Benlloch, Progress reports on the MEDAMI 2019 and CTR research at the DMIL in i3M. IL NUOVO CIMENTO, 43C (2020), 5.

4. E. Lamprou, F. Sanchez, J.M. Benlloch and A. J. Gonzalez, Exploring TOF capabilities of PET detector blocks based on large monolithic crystals and analog SiPMs. Physica Medica, 70 (2020), 10-18.

5. L. Moliner, V. Ilisie, A.J. Gonzalez, S. Olivera, A. Gonzalez-Montoro, V. GimenezAlventosa, G. Cañizares, E. Lamprou, J. Alamo, F. Sanchez, M.J. Rodriguez and J.M. Benlloch, TOF studies for dedicated PET with open geometries. Journal of Instrumentation, 14:2 (2019).

6. A. Gonzalez-Montoro, F. Sánchez, R. Martí, L. Hernández, A. Aguilar, J. Barberá, J.V. Catret, G. Cañizares, P. Conde, E. Lamprou, F. Martos, S. Sánchez, L.F. Vidal, J.M. Benlloch and A.J. González, Detector block performance based on a monolithic LYSO crystal using a novel signal multiplexing method. Nucl. Instrum. Methods Phys. Res. A., 912 (2018), 372-377.

7. E. Lamprou, A. Aguilar, A. González-Montoro, J.M. Monzó, G. Cañizares, S. Iranzo, L. F. Vidal, L. Hernández, P. Conde, S. Sánchez, F. Sánchez, A.J. González and J.M. 
Benlloch, PET detector block with accurate 4D capabilities. Nucl. Instrum. Methods Phys. Res. A., 912 (2018), 132-136.

8. A.J. Gonzalez, A. Gonzalez-Montoro, L.F. Vidal, J. Barbera, S. Aussenhofer, L. Hernandez, L. Moliner, F. Sanchez, C. Correcher, E. J. Pincay, G. Cañizares, E. Lamprou, S. Sanchez, J.V. Catret, S. Jiménez-Serrano, J. Cabello, M. Schwaiger, A. Iborra, T. Merlin, D. Visvikis and J.M. Benlloch, Initial Results of the MINDView PET Insert Inside the 3T mMR. IEEE Transactions on Radiation and Plasma Medical Sciences, 99:1 (2018), 343-35.

9. V. Ilisie, V. Giménez Alventosa, L. Moliner, A. Aguilar, E. Lamprou, A. González, F. Sánchez and J.M. Benlloch, Improving PET sensitivity with a Compton algorithm. Journal of Physics: Conference Series, 931 (2017).

10. A.J. Gonzalez, A. Gonzalez-Montoro, A. Aguilar, G. Cañizares, R. Martí, S. Iranzo, E. Lamprou, S. Sanchez, F. Sanchez and J. M. Benlloch, A scintillator geometry suitable for very small PET gantries. Journal of Instrumentation, 12:12 (2017).

11. J.M. Monzo, A. Aguilar, A. González-Montoro, E. Lamprou, A.J. González, L. Hernández, D. Mazur, R.J. Colom and J.M. Benlloch, PETIROC2 based readout electronics optimization for Gamma Cameras and PET detectors. Journal of Instrumentation, 12:2 (2017).

\section{Conference Proceedings}

1. J. Barrio, N. Cucarella, A.J. Gonzalez, S. Aguilar, A. Gonzalez-Montoro, V. Ilisie, E. Lamprou, F. Sanchez and J.M. Benlloch, Characterization of LYSO and CeBr3 Detectors with Lateral Sides Readout for a Multilayer Compton-PET. NSS/MIC 2019 - Conference Proceedings (2020).

2. E. Lamprou, A. Gonzalez-Montoro, G. Canizares, V. Ilisie, J. Barrio, F. Sanchez, A.J. Gonzalez and J.M. Benlloch, Characterization of TOF-PET Detectors Based on Monolithic Blocks and ASIC-Readout. IEEE Real Time 2018 - Conference Proceedings (2019).

3. A. Gonzalez-Montoro, E. Lamprou, A. Perez, L. Hernandez, G. Canizares, M. Freire, L. F. Vidal, E. J. Pincay, J. Barrio, S. Sanchez, F. Sanchez, J.M. Benlloch and A.J. Gonzalez, Reduction Signals Method Preserving Spatial and Temporal Capabilities. IEEE Real Time 2018 - Conference Proceedings (2019). 
4. E. Lamprou, A. González-Montoro, G. Cañizares, V. Ilisie, F. Sánchez, M. Freire, L. Hernández, L.F. Vidal, J. Barrio, A.J. González and J.M. Benlloch, TOF-PET Detectors Based on ASIC Technology and Analog SiPMs. NSS/MIC 2018 - Conference Proceedings (2019).

5. V. Ilisie, Giménez-Alventosa, L. Moliner, A. Aguilar, E. Lamprou, A.J. González, F. Sánchez and J.M. Benlloch, Improving PET Sensitivity and Resolution by Photon Interaction Sequence Timing Discrimination. NSS/MIC 2017 - Conference Proceedings (2018).

6. S. Sánchez, A. Iborra, P. Conde, A.J. González, J.M. Álvarez-Gómez, P. Bellido, A. González-Montoro, A. Aguilar, G. Cañizares, E. Lamprou, L. Moliner, F. Sánchez, J.M. Benlloch and M.J. Rodriguez Alvarez, A Direct Ray Tracing Reconstruction Algorithm Using an Adaptive Median Filter NSS/MIC 2017 - Conference Proceedings (2018).

7. E. Lamprou, A. Aguilar, A. González-Montoro, J.M. Monzó, G.Cañizares, L.F. Vidal, L. Hernández, S. Iranzo, R. Martí, S. Sánchez, F. Sánchez, A.J. González and J.M. Benlloch, Progress Report for an Accurate PET Detector Based on SiPMs and the TOFPET ASIC. NSS/MIC 2017 - Conference Proceedings (2018).

8. A. Aguilar, J.M. Monzó, A. J. González, A. González-Montoro, G. Cañizares, L.F. Vidal, L. Hernández, R. Colom, D. Grau-Ruíz, E. Lamprou, J. P. Rigla, E. Díaz-Caballero, J.J. García-Garrigós, P. Bellido, P. Conde, A. Iborra, L. Moliner, M. J. Rodríguez-Álvarez, S. Sánchez, M. Seimetz, A. Soriano, F. Sánchez and J.M. Benlloch, Preliminary characterization of ASIC-based detectors for TOF-PET applications. NSS/MIC 2016 Conference Proceedings (2017).

\section{Conferences and Workshops attendances}

- IEEE Nuclear Science Symposium and Medical Imaging Conference, Boston, USA, 2020 (Winner of the MIC Trainee Grant).

- IEEE Nuclear Science Symposium and Medical Imaging Conference, Manchester, UK, 2019.

- Mediterranean Thematic Workshop in Advanced Molecular Imaging (MEDAMI), Valencia, Spain, 2019. 
- IEEE Nuclear Science Symposium and Medical Imaging Conference, Manchester, UK, 2019.

- Cost Action, Bucharest, Romania, 2018.

- Cost Action, Ljubljana, Slovenia, 2018.

- $\quad$ Cost Action, Athens, Greece, 2018.

- IEEE Real Time Conference, Virginia, USA, 2018.

- $\quad$ Cost Action, Lisbon, Portugal, 2017.

- IEEE Nuclear Science Symposium and Medical Imaging Conference, Atlanta, USA, 2017.

- Conference on New Developments in Photodection (NDIP), Tours, France, 2017. (Winner of the young scientist best poster award for the session Blanc). 
